\title{
The role of mitochondria in the potential hepatotoxicity caused by cadmium telluride quantum dot nanoparticles
}

\author{
by \\ Kathy C. Nguyen
}

A thesis submitted to the Faculty of Graduate and Postdoctoral Affairs in partial fulfillment of the requirements for the degree of

\author{
Doctor of Philosophy \\ in \\ Biology, Specialization in Chemical and Environmental \\ Toxicology
}

Carleton University

Ottawa, Ontario

(C) 2015

Kathy C. Nguyen 


\section{Abstract}

Nanotechnology has widespread applications in a variety of fields, thus raising concerns about the potential health risk of nanomaterials. Nanoparticle (NP) toxicity is often linked to their novel physiochemical properties which allow them to be transported across cell membranes or to be taken up into target cells, causing changes in sub-cellular structures and affecting cellular activity. This thesis focused on quantum dots (QDs) which are engineered NPs of semiconductor structures. Cadmium-based QDs such as cadmium telluride QDs (CdTe-QDs), owing to their unique optical and electronic properties, have gained much interest as emerging fluorophores and are widely used in microelectronic and biomedical applications. However, the toxicity associated with these NPs has been a concern and needs to be thoroughly investigated. The overall objectives of this thesis were 1) to examine hepatotoxicity of CdTe-QDs in a hepatocyte cell line and in a mouse model and 2) to investigate mitochondrial effects as the underlying mechanisms of CdTe-QD hepatotoxicity. In HepG2 cells, CdTe-QDs induced cytotoxicity and oxidative stress by increasing reactive oxygen species production and interfering with antioxidant defenses, ultimately activating extrinsic and intrinsic apoptosis pathways. CdTe-QDs also caused mitochondrial toxicity, including mitochondrial enlargement, membrane potential disruption, electron transport chain (ETC) complexes alterations, decreased cellular respiration, ATP depletion, and mitochondrial biogenesis activation. Exposure of BALB/c mice to CdTe-QDs via intravenous injection revealed that the liver was the main target tissue for toxicity. Hepatotoxicity was evidenced by changes in the liver tissue architecture and liver injury biomarkers. At the cellular level, CdTe-QDs induced liver oxidative stress and apoptosis. 
Effects on mitochondria of liver hepatocytes were also observed, including enlargement and increased number of these organelles and effects on ETC complexes and ATP synthesis. This thesis demonstrates that mitochondria are the prominent targets for CdTe-QD hepatotoxicity as mitochondrial dysfunction resulted in energy metabolism impairment and intracellular oxidant stress that signaled apoptotic pathways, leading to observed cytotoxicity and liver damage in the test models. This thesis provides insights into the toxicological mechanisms of QDs in biological systems and helpful information towards bridging the gap between in vitro and in vivo testing for risk assessment of these NPs. 


\section{Acknowledgements}

I would like to take this opportunity to thank all the people who over the years contributed directly or indirectly to this work and provided help and support in many ways to make this thesis possible and an unforgettable experience for me.

First of all, I would like express my deep and sincere gratitude to my supervisors, Dr. Azam Tayabali (Health Canada, Carleton University) and Dr. William Willmore (Carleton University), who have provided me with much guidance, encouragement, and support throughout my whole graduate studies (Master and Ph.D.). I am very grateful for their dedication of time, valuable suggestions, and helpful and brainstorming discussions during the progress of my thesis. Dr. Willmore and Dr. Tayabali have been a great influence in many aspects of my graduate experience over the past five years.

My sincere thanks and appreciation go to my thesis advisors, Dr. Thomas Moon (University of Ottawa) and Dr. Yves Aubin (Health Canada, Carleton University), for their insightful feedbacks, helpful advice, support, and time on my research project. Also, special thanks go to Dr. Vern Seligy (Health Canada) and Dr. Ki-Young Lee (University of Calgary), who encouraged me to pursue graduate studies and has given great support throughout the years.

I am grateful to the animal care staff in the Scientific Services Division, Bureau of Chemical Safety, Health Canada, especially Dr. Martha Navarro, Julie Todd, Kelvin Kittle, Dominique Patry, Douglas Parks, Michelle Lalande, and Laura Samson, for their invaluable contributions to the animal work of this thesis. I would like to thank Peter Rippstein and Junhui Tan (Heart Institute, University of Ottawa) for performing TEM preparation and analysis, Lorraine Casavant (Environmental Health Science and Research 
Bureau) and Dr. Don Caldwell (Bureau of Chemical Safety) for their help with tissue histology work and analysis, Dr. Andrey Massarsky (University of Ottawa) for performing Dynamic Light Scattering measurement for nanoparticle characterization, Emmanuel Yumvihoze, Dr. Alexandre Poulain (University of Ottawa), and the staff of Intertek Allentown for their help with ICP-MS and ICP-OES ananlyis.

My many thanks go to friends, colleagues, post-docs and students at Health Canada, especially Dr. Elyse Bernard, Dr. Sami Qutob, Dr. Mary Zhang, Rebecca Maertens, Andrea Rowan-Carroll, Dong-Mei Wu, Jennifer Crosthwait, Gordon Coleman, Dr. Phil Shwed, Alexandra Long, Julie Cox, Agnieszka Bielecki, Julie Buick, Dr. Carole Yauk, Lynda Soper, and Laura Richards, for their technical assistance and/or support in different ways, during my part- time studies to pursue the Ph.D. degree. I would also like to thank Drs. Hongyan Dong, David Lefebvre, Sabina Halappanavar, and Francesco Marchetti for their reviews of my manuscripts of this research work.

I would like to acknowledge the Mechanistic Studies Division, Environmental Health Science and Research Bureau, and Health Canada for providing me with opportunities to pursue my graduate studies part-time. An additional thank to my work supervisor, Dr. Azam Tayabali, and many thanks to Dr. Michele Regimbald-Krnel (divisional manager) and Tim Singer (bureau director) for financial support to this research project.

Finally, a special gratitude and love goes to my family and close friends for the continuing love, care, and support they have given in all things that I do. I am eternally grateful for their emotional support and encouragement throughout my Ph.D. studies and for their confidence in my pursuit. 


\section{Preface}

This section contains full bibliographical details and statements of contributions of two published articles and two manuscripts included in this thesis. The content of the articles and manuscripts has been adapted to fit in the thesis structure. Use of copyrighted material is acknowledged in this section.

In accordance to the Integrated Thesis policy of Carleton University, the supervisors (Dr. William G. Willmore and Dr. Azam F. Tayabali) and the author of the thesis (Kathy C. Nguyen) confirm that the student was fully involved in designing, setting up, and conducting the research, collecting and obtaining data, analyzing results, and preparing and writing the co-authored articles and manuscripts integrated in the thesis.

\section{Chapter 2}

This chapter is based upon the following published article:

Kathy C. Nguyen, William G. Willmore, and Azam F. Tayabali. (2013). Cadmium Telluride Quantum Dots Cause Oxidative Stress Leading to Extrinsic and Intrinsic Apoptosis in Hepatocellular Carcinoma HepG2 Cells. Toxicology 306: 114-123.

Permission was obtained from Toxicology journal and Government of Canada for incorporation of this paper into this thesis. Minor changes were performed to the formatting of the document so that it could fit in the format of this dissertation.

For this paper, Kathy C. Nguyen designed and conducted these experiments, and wrote the manuscript. Drs. Azam Tayabali and William Willmore provided input into the manuscript. Dr. Azam Tayabali and Health Canada provided funding for the project. 


\section{Chapter 3}

This chapter is based upon the following published article:

Kathy C. Nguyen, Peter Rippstein, Azam F. Tayabali, and William G. Willmore. (2015). Mitochondrial Toxicity of Cadmium Telluride Quantum Dot Nanoparticles in Mammalian Hepatocytes. Toxicological Sciences 146(1): 31-42

Permission was obtained from the Toxicological Sciences journal for incorporation of this paper into this thesis. Minor changes were performed to the formatting of the documemt so that it could fit in the format of this dissertation.

For this paper, Kathy C. Nguyen designed and conducted these experiments and wrote the manuscript. Peter Ripstein (Heart Institute, University of Ottawa) performed TEM work. Drs. William Willmore and Azam Tayabali provided input into the manuscript. Dr. Azam Tayabali and Health Canada provided funding for the project. Dr. William Willmore provided funding for coloured figures in print versions of the paper.

\section{Chapter 4}

This chapter is based upon the following manuscript:

Kathy C. Nguyen, Yan (Mary) Zhang, Julie Todd, Kelvin Kittle, Douglas Parks, Dominique Patry, Lorraine Casavant, Don Caldwell, Emmanuel Yumvihoze, Michelle Lalande, Martha Navarro, Andrey Massarsky, Alexandre Poulain, Thomas W. Moon, William G. Willmore and Azam F. Tayabali. (2015). Biodistribution and Toxic Effects of Cadmium Telluride Quantum Dot Nanoparticles in a Mouse Model. To be submitted to Nanomedicine journal. 
For this manuscript, Kathy C. Nguyen designed and conducted these experiments and wrote the manuscript. Yan (Mary) Zhang assisted with animal necropsies. Julie Todd, Kelvin Kittle, Douglas Parks, Michelle Lalande, Dr. Martha Navarro assisted with animal exposure and necropsies. Dominique Patry performed hematology analysis. Lorraine Casavant, and Dr. Don Caldwell performed histology work. Emmanuel Yumvihoze and Dr. Alexandre Poulain assisted with ICP-Ms analysis. Dr. Andrey Massarsky and Dr. Thomas Moon assisted with DLS analysis. Drs. Azam Tayabali and William Willmore provided input into the manuscript. Dr. Azam Tayabali and Health Canada provided funding for the project.

\section{Chapter 5}

This chapter is based upon the following manuscript:

Kathy C. Nguyen, Yan (Mary) Zhang, Julie Todd, Douglas Parks, Kelvin Kittle, Martha Navarro, Azam F. Tayabali and William G. Willmore. (2015). In Vivo Hepatotoxicity of Cadmium Telluride Quantum Dot Nanoparticles, Mitochondrial Effects as a Mechanism. To be submitted to Nanotoxicology journal.

For this manuscript, Kathy C. Nguyen designed and conducted these experiments and wrote the manuscript. Yan Zhang assisted with the animal necropsies. Julie Todd, Douglas Parks, Kelvin Kittle, Michelle Lalande, and Martha Navarro assisted with the animal exposures and necropsies. Drs. William Willmore and Azam Tayabali provided input into the manuscript. Dr. Azam Tayabali and Health Canada provided funding for the project. 


\section{Table of Contents}

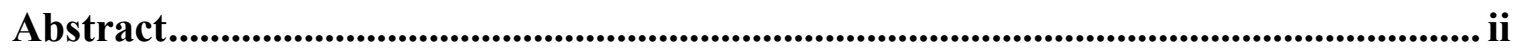

Acknowledgements ........................................................................................................................... iv

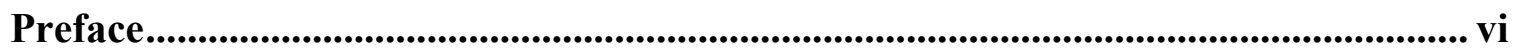

Table of Contents ........................................................................................................................... ix

List of Tables ............................................................................................................................. Xv

List of Figures................................................................................................................................. xvi

List of Abbreviations ............................................................................................................ xxix

1 Chapter: Introduction ............................................................................................................ 1

1.1 Nanomaterials and potential health impacts of nanoparticles ..................................... 1

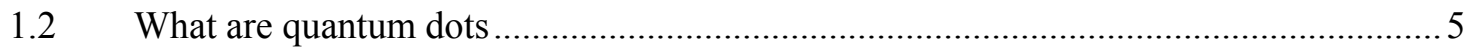

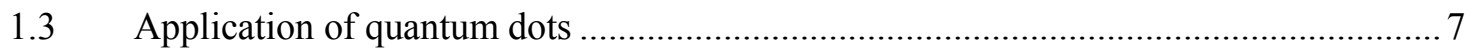

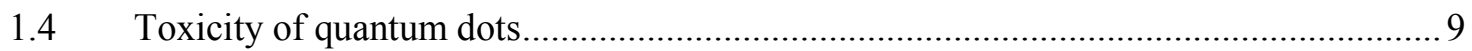

1.4.1 In vitro toxicity and proposed mechanisms......................................................

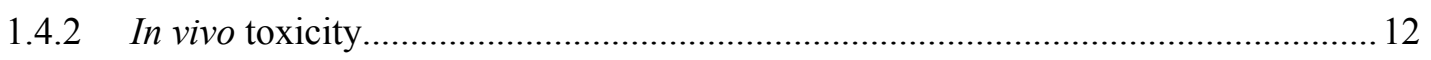

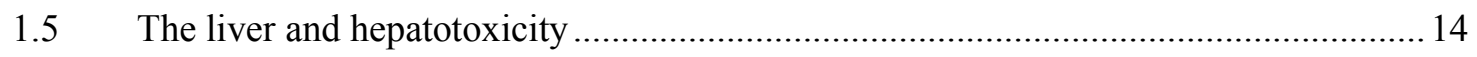

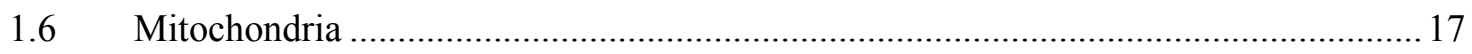

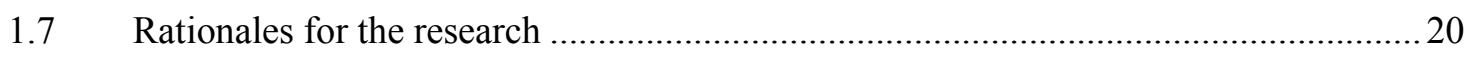

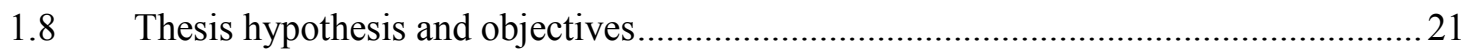

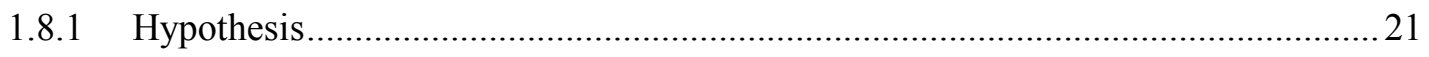

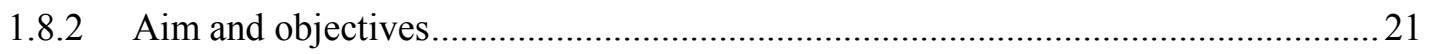

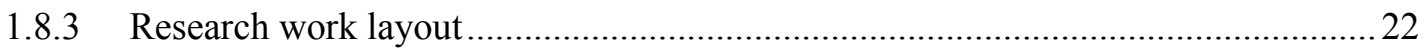

2 Chapter: Cadmium Telluride Quantum Dots Cause Oxidative Stress Leading to Extrinsic and Intrinsic Apoptosis in Hepatocellular Carcinoma HepG2 Cells......... 24 


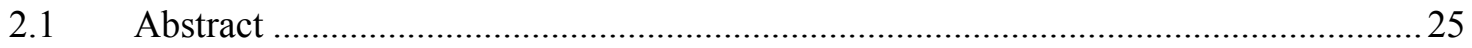

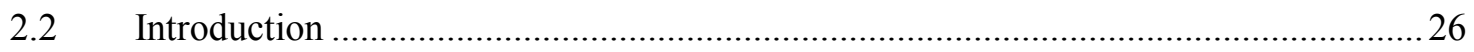

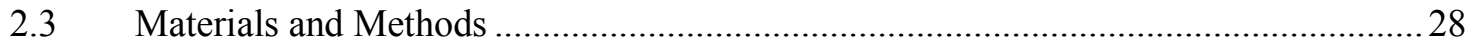

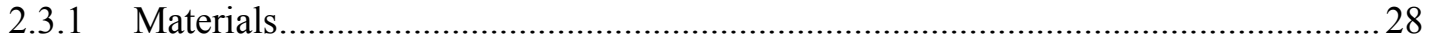

2.3.2 Spectral and size characterization of CdTe-QDs ..................................................29

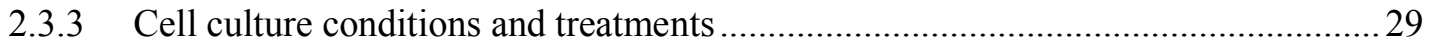

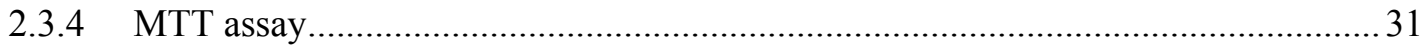

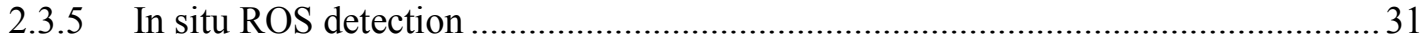

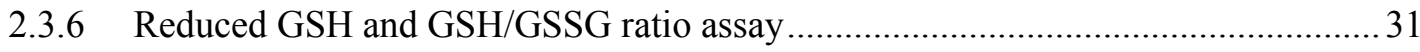

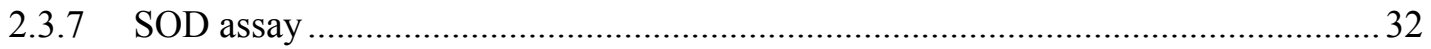

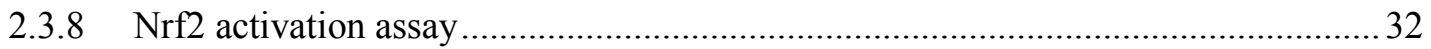

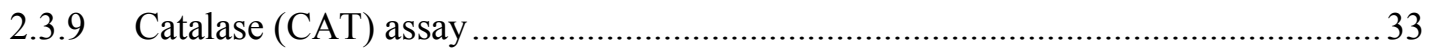

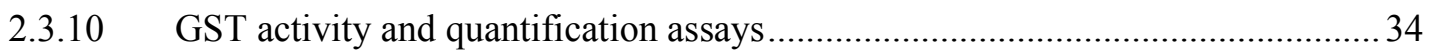

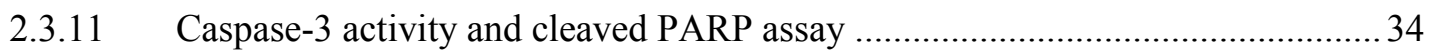

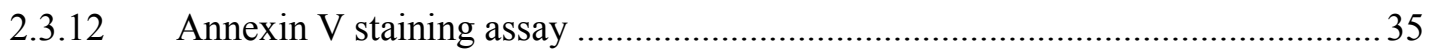

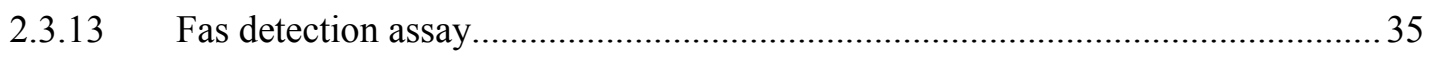

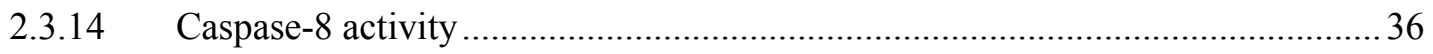

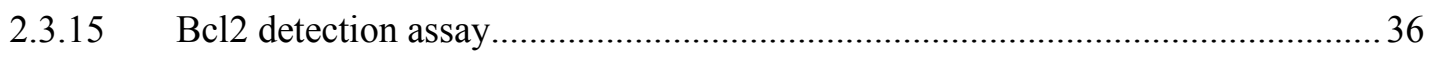

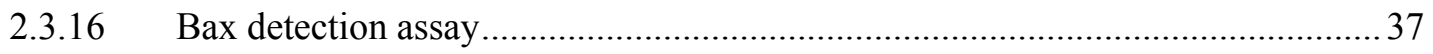

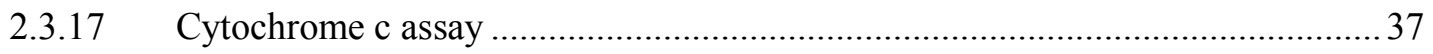

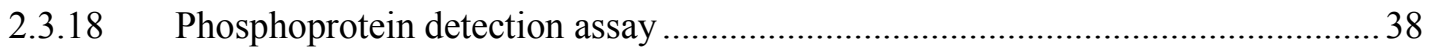

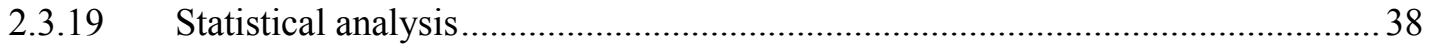

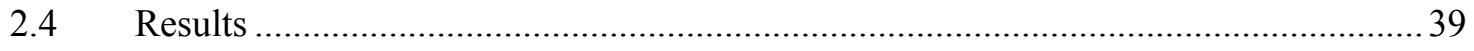

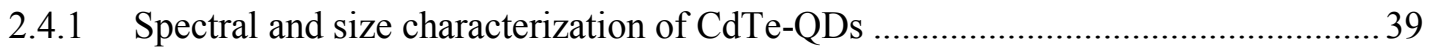

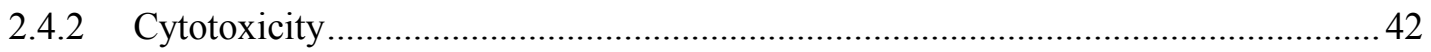

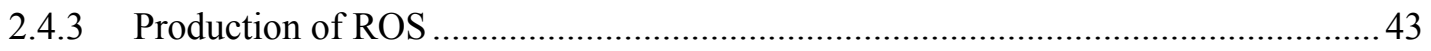




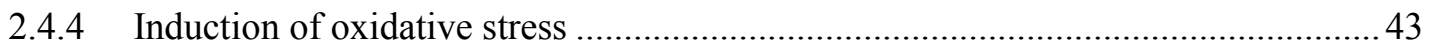

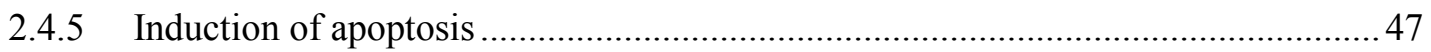

2.4.6 Effects of CdTe-QDs on biomarkers of extrinsic apoptotic pathway ..................... 49

2.4.7 Effects of CdTe-QDs on biomarkers of intrinsic apoptotic pathway .......................51

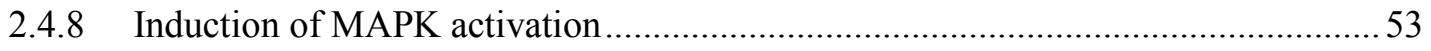

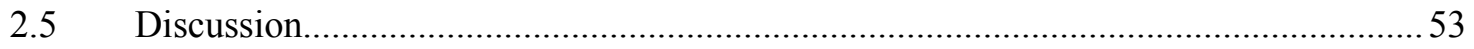

3 Chapter: Mitochondrial Toxicity of Cadmium Telluride Quantum Dot Nanoparticles in Mammalian Hepatocytes............................................................. 61

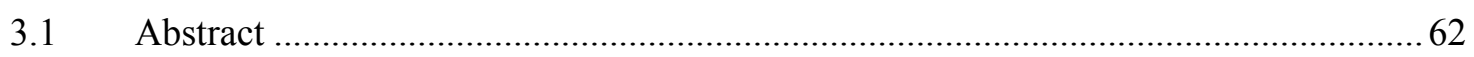

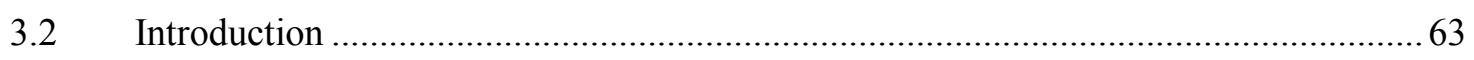

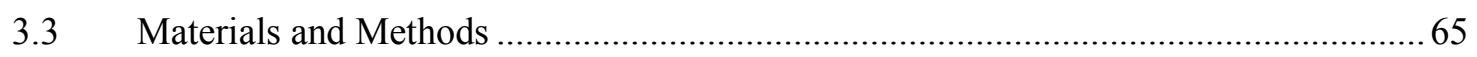

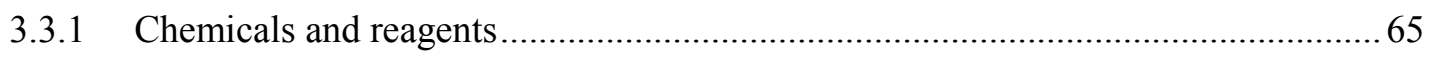

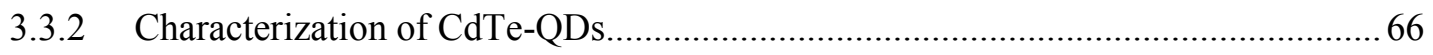

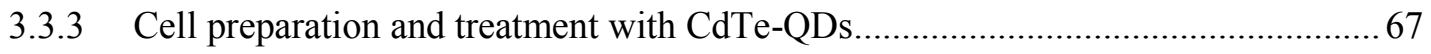

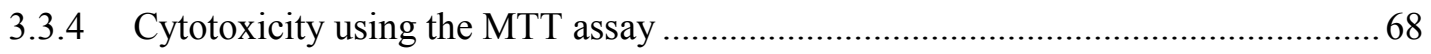

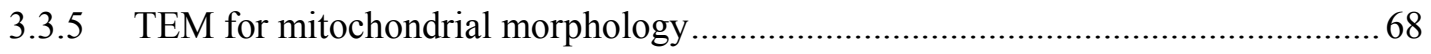

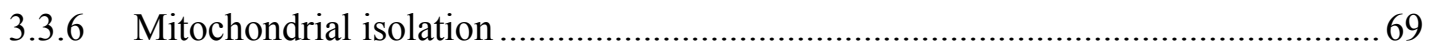

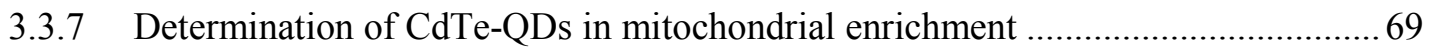

3.3.8 Mitochondrial membrane potential measurements by confocal microscopy ...........69

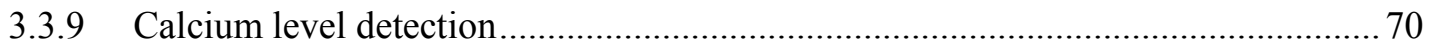

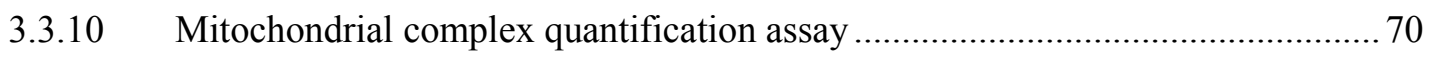

3.3.11 Mitochondrial complex activity assay ….................................................... 71

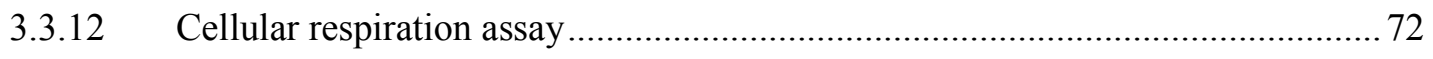

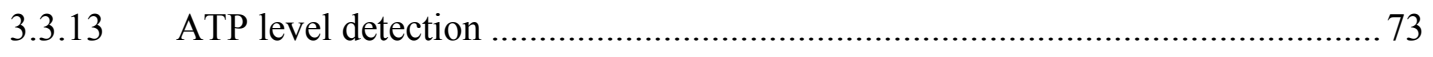

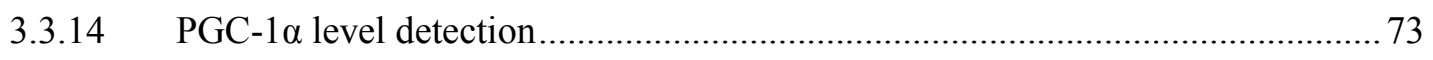

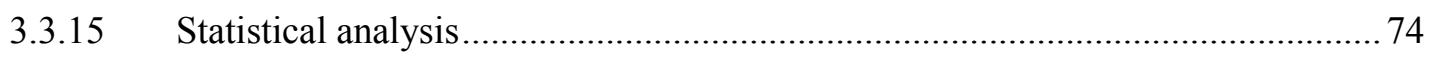




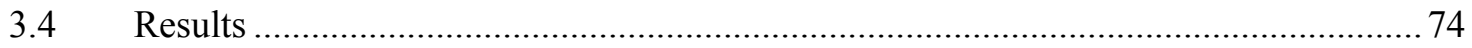

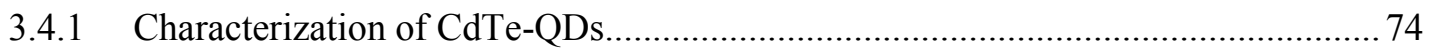

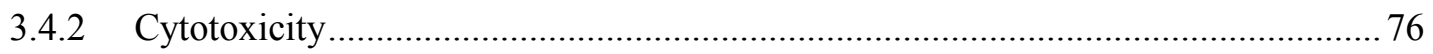

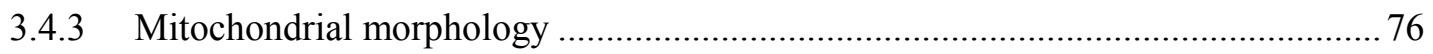

3.4.4 Detection of CdTe-QDs in isolated mitochondria................................................. 79

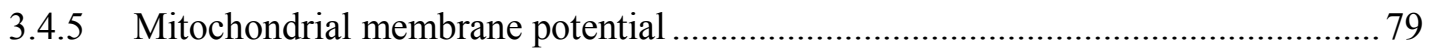

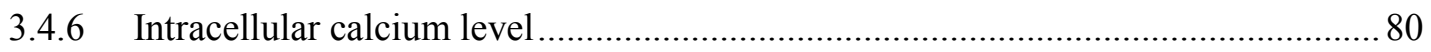

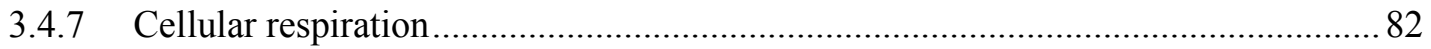

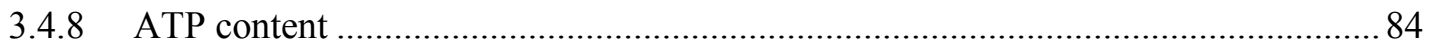

3.4.9 Electron transport chain component enzyme level and activity .............................. 85

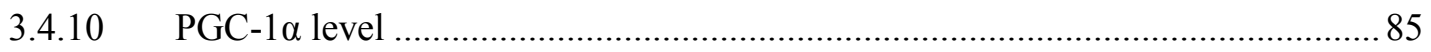

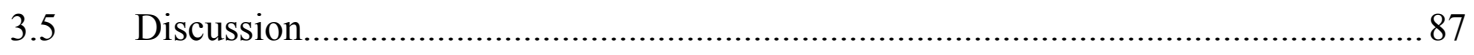

4 Chapter: Biodistribution and systemic effects of cadmium telluride quantum dot nanoparticles in a mouse model after intravenous administration ........................... 97

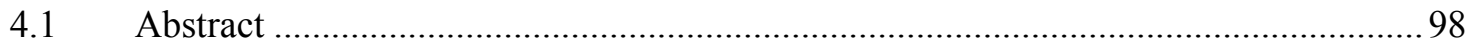

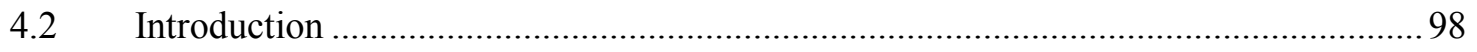

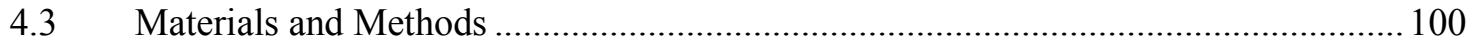

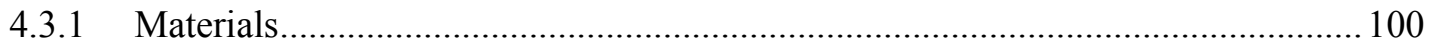

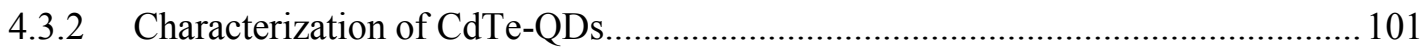

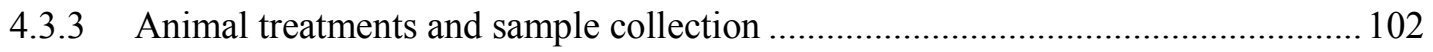

4.3.4 Inductively coupled plasma mass spectrometry (ICP-MS) .................................... 104

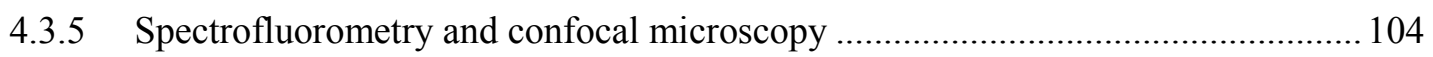

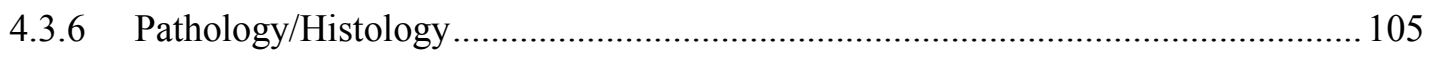

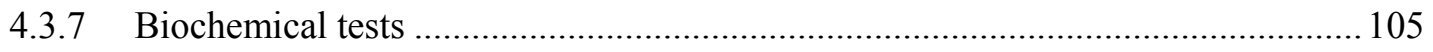

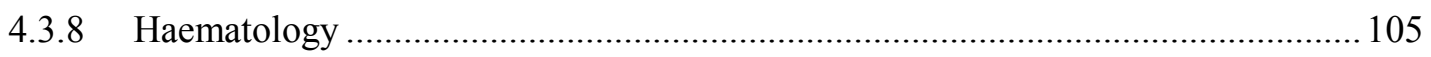

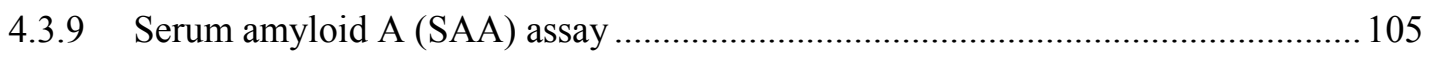




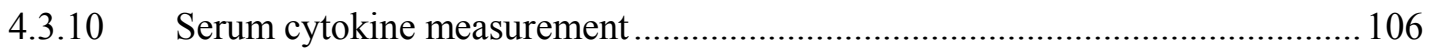

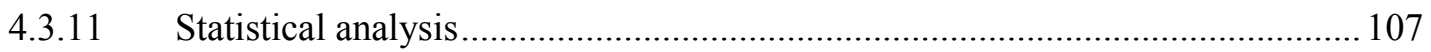

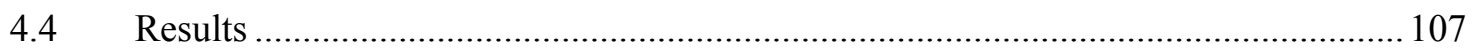

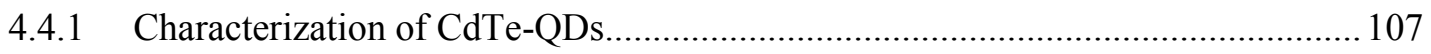

4.4.2 Detection of cadmium in blood and tissues using ICP-MS................................... 109

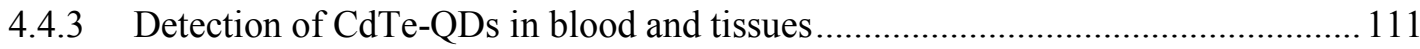

4.4.4 Effects of CdTe-QDs on behaviour and physical appearance of mice....................113

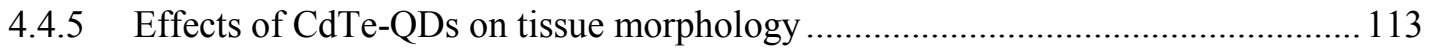

4.4.6 Changes in biochemical parameters ….......................................................... 115

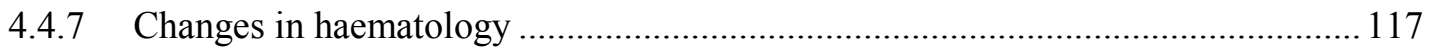

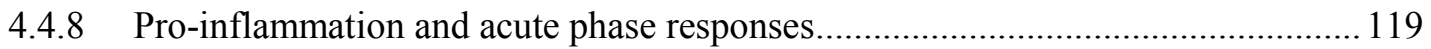

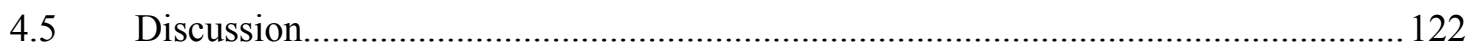

\section{Chapter: In Vivo Hepatotoxicity of Cadmium Telluride Quantum Dot}

Nanoparticles - Mitochondrial Effects As a Mechanism................................................ 130

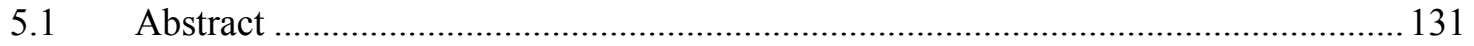

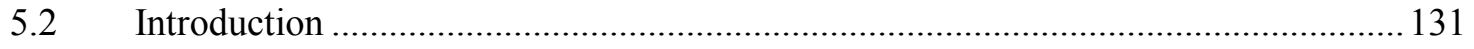

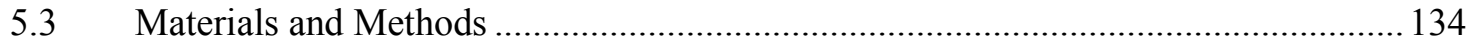

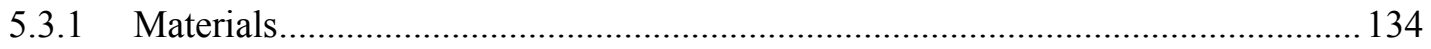

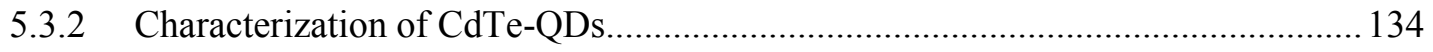

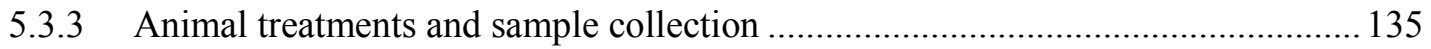

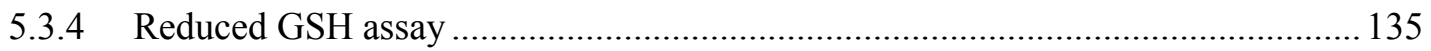

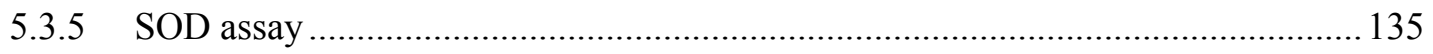

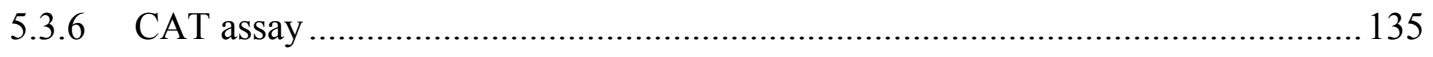

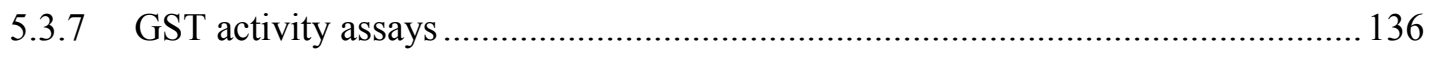

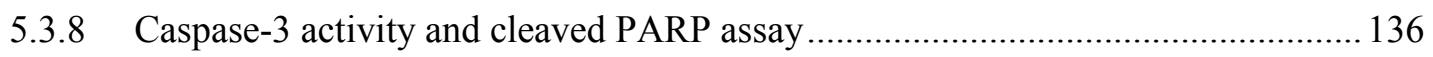

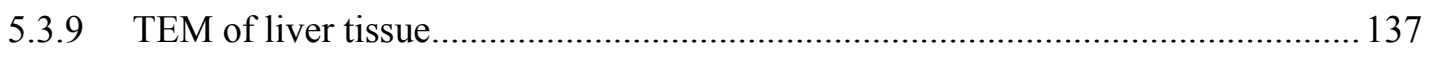


5.3.10 Mitochondrial complex quantification assay

5.3.11 RNA isolation and gene expression analyses

5.3.12 ATP level detection

5.3.13 PGC-1 $\alpha$ level detection.

5.3.14 Statistical analysis. 140

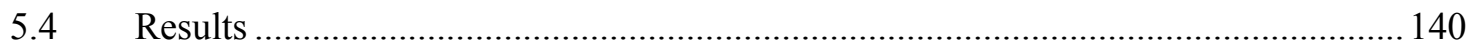

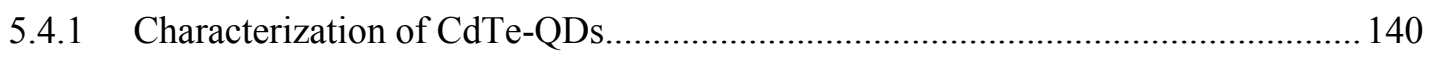

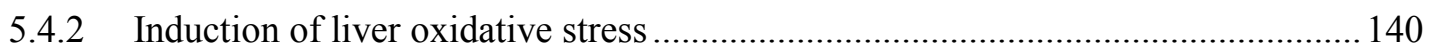

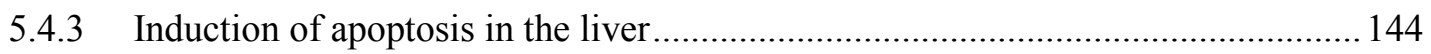

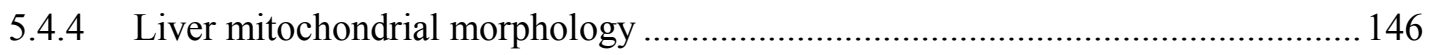

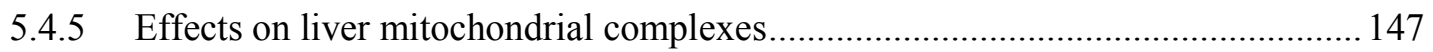

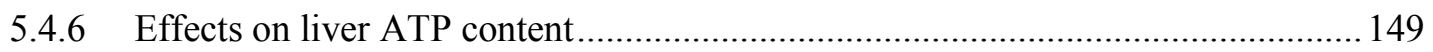

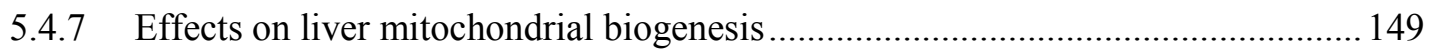

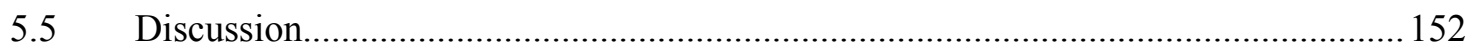

6 Chapter: General Discussion and Conclusion .................................................. 160

6.1 Summary of research rationales, hypothesis, and approaches .................................. 160

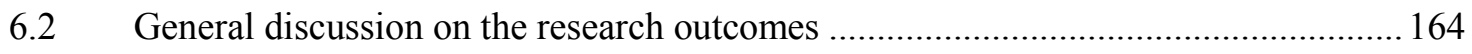

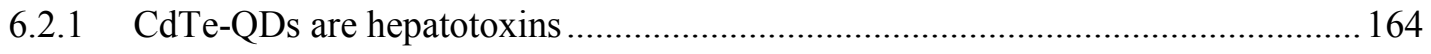

6.2.2 Cadmium is not the only factor that contributes to CdTe-QD hepatotoxicity ....... 166

6.2.3 Mitochondria are the prime targets and mitochondrial toxicity is the mechanism of

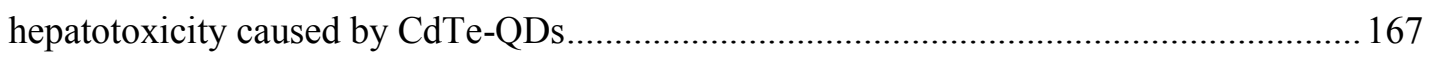

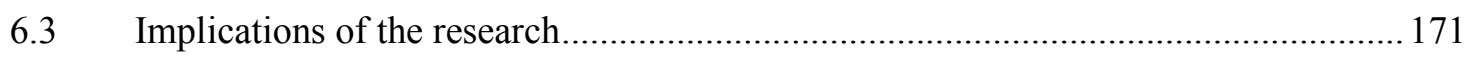

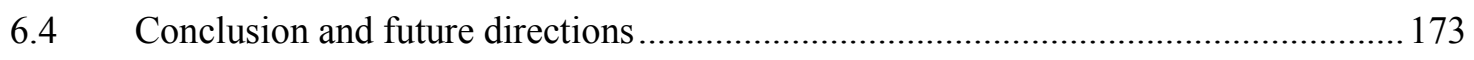

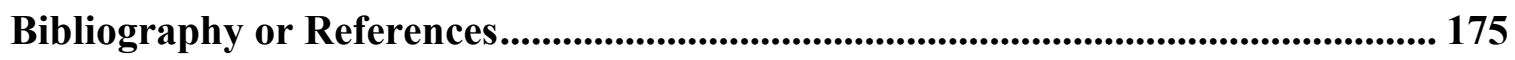




\section{List of Tables}

\section{Chapter 3}

Table 3.1. ICP-OES analysis for determination of elements in CdTe-QDs. Elements of interest were confirmed using a minimum of two analytical wavelengths for each element. Five replicate readings were performed on each analyte. 75

\section{Chapter 4}

Table 4.1. Cadmium concentrations ( $\mu \mathrm{g} / \mathrm{kg}$ tissue) in the blood and tissues as detected by ICP-MS. Mice were treated with $5 \mathrm{mg} / \mathrm{kg}$ bw of CdTe-QDs for different time points.

Table 4.2. Comparison of cadmium levels in CdTe-QD- and $\mathrm{CdCl}_{2}$-treated blood and tissues. Mice were treated with $6 \mathrm{mg} / \mathrm{kg}$ bw of CdTe-QDs or $1.95 \mathrm{mg} / \mathrm{kg}$ bw of $\mathrm{CdCl}_{2}$ which contains an equivalent cadmium concentration, and their blood and tissues examined at $24 \mathrm{~h}$ post-exposure 


\section{List of Figures}

\section{Chapter 1}

Figure 1.1. Basic structure of a core-shell QD and a core-shell QD conjugated with

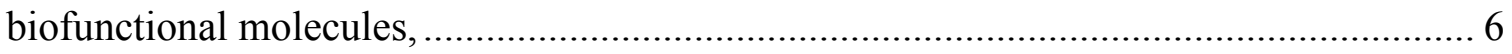

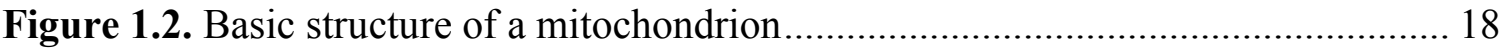

\section{Chapter 2}

Figure 2.1. Characterization of CdTe-QDs. (A) Fluorescence spectra for CdTe-QDs at $450 \mathrm{~nm}$ excitation and emission wavelengths from $500 \mathrm{~nm}$ to $580 \mathrm{~nm}$. (B) Standard curves for CdTe-QDs at $485 \mathrm{~nm}$ excitation and $540 \mathrm{~nm}$ emission. The standard curve was plotted using linear curve fit $(y=A+B x)$ with $A=21.056, B=43.995$, and $R^{2}=0.997$. (C) AFM image of full commercial concentration of CdTe-QD deposited on freshly cleaved mica. (D) Summary of Z-dimension size measurements of diluted CdTe-QDs as measured by AFM. (E) TEM image of diluted CdTe-QDs. (F) The diameter of each individual CdTe-QDs was measured at its minimum and maximum dimensions. 41

Figure 2.2. Cytotoxicity in HepG2 cells as assessed by loss of MTT bioreduction activity of CdTe-QD treated cultures relative to PBS treated controls. Data points represent the means of three independent experiments done in duplicate \pm standard deviations. The asterisks $(*)$ indicate statistically significant differences compared to PBS-treated control $(\mathrm{p}<0.001)$.

Figure 2.3. Confocal micrographs showing ROS production in HepG2 cells. Representative micrographs of cells treated with (A) PBS, (B) $10 \mu \mathrm{g} / \mathrm{ml}$ CdTe-QDs (containing $1 \mu \mathrm{g} / \mathrm{ml}$ of cadmium) for $24 \mathrm{~h},(\mathbf{C}) 1.63 \mu \mathrm{g} / \mathrm{ml}$ of $\mathrm{CdCl}_{2}$ (containing $1 \mu \mathrm{g} / \mathrm{ml}$ 
of cadmium), and (D) menadione ( $25 \mu \mathrm{M})$, and labeled with DHE. The presence of ROS is indicated with red fluorescence. (E) The total area of red fluorescence (pixels squared: $\mathrm{px}^{2}$ ) was analysed with image analysis software (NIS Elements) using three micrographs for each sample. Each data point represents the mean \pm standard deviation. The asterisks $\left(^{*}\right)$ indicate statistically significant differences compared to PBS-treated control $(\mathrm{p}<0.001)$. The number $(\#)$ sign indicates statistically significant differences compared to the CdTe-QD-treated group $(\mathrm{p}<0.05)$ 45

Figure 2.4. Effects of CdTe-QDs on biomarkers of oxidative stress. Cells were treated with $10 \mu \mathrm{g} / \mathrm{ml} \mathrm{CdTe-QDs} \mathrm{(containing} 1 \mu \mathrm{g} / \mathrm{ml}$ of cadmium) or $1.63 \mu \mathrm{g} / \mathrm{ml}$ of $\mathrm{CdCl}_{2}$ (containing $1 \mu \mathrm{g} / \mathrm{ml}$ of cadmium) for $24 \mathrm{~h}$. (A) GSH level as measured using an enzymatic assay, (B) GSH/GSSG ratio, (C) SOD activity as measured using enzymatic assay and (D) Nrf2 activation as measured using ELISA assay and represented by optical density at $450 \mathrm{~nm}\left(\mathrm{OD}_{450}\right) \cdot \mathrm{CdCl}_{2}$ was used for comparison. Data points represent the means of three independent experiments done in duplicate \pm standard deviations. The asterisks $(*)$ indicate statistically significant differences compared to control with $\mathrm{p}<0.001$. The number (\#) signs indicate statistically significant differences compared to the CdTe-QD-treated group $(\mathrm{p}<0.05)$. 46

Figure 2.5. Effects of CdTe-QDs on antioxidant enzymes. Cells were treated with 10 $\mu \mathrm{g} / \mathrm{ml} \mathrm{CdTe}$-QDs (containing $1 \mu \mathrm{g} / \mathrm{ml}$ of cadmium) or $1.63 \mu \mathrm{g} / \mathrm{ml}$ of $\mathrm{CdCl}_{2}$ (containing 1 $\mu \mathrm{g} / \mathrm{ml}$ of cadmium) for $24 \mathrm{~h}$. (A) GST activity and concentration as measured using enzymatic and ELISA assays, respectively, (B) CAT activity as measured using enzymatic assay. $\mathrm{CdCl}_{2}$ was used for comparison. Data points represent the means of 
three independent experiments done in duplicate \pm standard deviations. The asterisks $(*)$ indicate statistically significant differences compared to PBS-treated control $(\mathrm{p}<0.001) .47$ Figure 2.6. Effects of CdTe-QDs on biomarkers of apoptosis. Cells were treated with 10 $\mu \mathrm{g} / \mathrm{ml} \mathrm{CdTe-QDs} \mathrm{(containing} 1 \mu \mathrm{g} / \mathrm{ml}$ of cadmium) or $1.63 \mu \mathrm{g} / \mathrm{ml}$ of $\mathrm{CdCl}_{2}$ (containing 1 $\mu \mathrm{g} / \mathrm{ml}$ of cadmium) for $24 \mathrm{~h}$. (A) Active caspase-3 level and (B) cleaved PARP level were measured using bead plex assays and data were represented by fluorescence intensities (FI). $\mathrm{CdCl}_{2}$ was used for comparison. STS $(1 \mu \mathrm{M})$ was used as a positive control. Data points represent the means of three independent experiments done in duplicate \pm standard deviations. The asterisks $(*)$ indicate statistically significant differences compared to PBS-treated control $(\mathrm{p}<0.001)$. The number $(\#)$ signs indicate statistically significant differences compared to the CdTe-QD-treated group $(\mathrm{p}<0.05)$. . 48

Figure 2.7. Confocal micrographs showing externalization of phosphatidylserine (PS) in HepG2 cells induced by CdTe-QDs. Cells were treated with (A) PBS, (B) $10 \mu \mathrm{g} / \mathrm{ml}$ CdTe-QDs (containing $1 \mu \mathrm{g} / \mathrm{ml}$ of cadmium) for $24 \mathrm{~h}$, (C) $1.63 \mu \mathrm{g} / \mathrm{ml}$ of $\mathrm{CdCl}_{2}$ (containing $1 \mu \mathrm{g} / \mathrm{ml}$ of cadmium), and (D) STS $(1 \mu \mathrm{M})$, and were then stained with annexin V-PE. The binding of annexin V to externalized PS exhibited red fluorescence. Nuclei were stained with Sytox Red ${ }^{\mathrm{TM}}$ and exhibit blue fluorescence. 49

Figure 2.8. Effects of CdTe-QDs on biomarkers of extrinsic apoptosis. Cells were treated with $10 \mu \mathrm{g} / \mathrm{ml} \mathrm{CdTe-QDs} \mathrm{(containing} 1 \mu \mathrm{g} / \mathrm{ml}$ of cadmium) or $1.63 \mu \mathrm{g} / \mathrm{ml}$ of $\mathrm{CdCl}_{2}$ (containing $1 \mu \mathrm{g} / \mathrm{ml}$ of cadmium) for $24 \mathrm{~h}$. (A) Fas level as measured using EIA assay and (B) Caspase 8 activity as measured using enzymatic assay and represented by optical density at $450 \mathrm{~nm}\left(\mathrm{OD}_{450}\right)$. STS $(1 \mu \mathrm{M})$ was used as a positive control. Data points represent the means of three independent experiments done in duplicate \pm standard 
deviations. The asterisks $(*)$ and $(* *)$ indicate statistically significant differences compared to control with $p<0.001$ and with $p<0.05$, respectively. The number (\#) signs indicate statistically significant differences compared to the CdTe-QD-treated group $(\mathrm{p}<0.05)$ 50

Figure 2.9. Effects of CdTe-QDs on biomarkers of the intrinsic apoptotic pathway and MAPKs. Cells were treated with $10 \mu \mathrm{g} / \mathrm{ml} \mathrm{CdTe-QDs} \mathrm{(containing} 1 \mu \mathrm{g} / \mathrm{ml}$ of cadmium) or $1.63 \mu \mathrm{g} / \mathrm{ml}$ of $\mathrm{CdCl}_{2}$ (containing $1 \mu \mathrm{g} / \mathrm{ml}$ of cadmium) for $24 \mathrm{~h}$. (A) Bcl2 level as measured using bead plex assay and represented by fluorescence intensity (FI), (B) Bax level as measured using EIA assay, (C) Cytosolic and mitochondrial cytochrome c as measured using ELISA kit and (D) phosphorylated Erk (1/2), JNK and p38 levels as measured using bead plex assay and represented by fluorescence intensity (FI). STS (1 $\mu \mathrm{M})$ was as a positive control. Data points represent the means of three independent experiments done in duplicate \pm standard deviations. The asterisks $(*)$ and $(* *)$ indicate statistically significant differences compared to control with $\mathrm{p}<0.001$ and with $\mathrm{p}<0.05$, respectively. The number (\#) signs indicate statistically significant differences compared to the CdTe-QD-treated group $(\mathrm{p}<0.05)$. 52

\section{Chapter 3}

Figure 3.1. Cytotoxicity in HepG2 cells as assessed by loss of MTT bioreduction activity of CdTe-QDs, $\mathrm{CdCl}_{2}$, and $\mathrm{Na}_{2} \mathrm{TeO}_{3}$ treated cultures relative to PBS treated controls. (A) Cells were treated with different concentrations of CdTe-QD and $\mathrm{CdCl}_{2}$, at equivalent concentrations of cadmium, for 6,12 , and $24 \mathrm{~h}$. The concentrations of CdTeQDs and $\mathrm{CdCl}_{2}$ were expressed in cadmium concentrations. (B) Cells were treated with 
different concentrations of $\mathrm{Na}_{2} \mathrm{TeO}_{3}$ for 6,12 , and $24 \mathrm{~h}$. The concentrations of $\mathrm{Na}_{2} \mathrm{TeO}_{3}$ were expressed in tellurium concentrations that corresponded to the concentrations of cadmium in CdTe-QDs used in (A). Data points represent the means of three independent experiments done in duplicate \pm standard deviations. The asterisks $\left(^{*}\right)$ indicate statistically significant differences compared to PBS-treated control $(\mathrm{p}<0.05)$. The number (\#) sign indicates statistically significant differences of $\mathrm{CdCl}_{2}$ compared to the CdTe-QD-treated group $(\mathrm{p}<0.05)$. 77

Figure 3.2. Transmission electron micrographs of HepG2 cells showing the effects of CdTe-QDs on mitochondrial morphology and structure. Cells were treated with $10 \mu \mathrm{g} / \mathrm{ml}$ CdTe-QDs (containing $1 \mu \mathrm{g} / \mathrm{ml}$ of cadmium) for $24 \mathrm{~h}$. (A) and (C) PBS-treated control at low and high magnifications. (B) and (D) CdTe-QD treated cells at low and high magnifications. (n) nuclear, (m) mitochondrion. 78

Figure 3.3. Fluorometric detection of CdTe-QDs in enriched mitochondrial fractions. Fluorescence intensity was measured at an excitation of $485 \mathrm{~nm}$ and emission of $540 \mathrm{~nm}$. Each data point represents the mean \pm standard deviation. The asterisks $(*)$ indicate statistically significant differences compared with the PBS-treated control $(\mathrm{p}<0.05)$.... 79

Figure 3.4. Confocal micrographs of HepG2 cells showing the effects on mitochondrial membrane potential induced by $\mathrm{CdTe}-\mathrm{QDs}$ and $\mathrm{CdCl}_{2}$, at an equivalent concentration of cadmium (1 $\mu \mathrm{g} / \mathrm{ml}$ ), using TMRE staining. (A) PBS-treated control, (B) CdTe-QDs, which appeared as green dots/aggregates on the micrograph, (C) $\mathrm{CdCl}_{2}$, and (D) $\mathrm{CCCP}$, as a positive control. 80

Figure 3.5. Confocal micrographs showing intracellular $\mathrm{Ca}^{2+}$ levels in $\mathrm{HepG} 2$ cells. (A) PBS-treated control, (B) cells treated with $10 \mu \mathrm{g} / \mathrm{ml} \mathrm{CdTe-QDs} \mathrm{(containing} 1 \mu \mathrm{g} / \mathrm{ml}$ of 
cadmium) for $24 \mathrm{~h},(\mathbf{C})$ cells treated with $1.63 \mu \mathrm{g} / \mathrm{ml} \mathrm{CdCl}$ (containing $1 \mu \mathrm{g} / \mathrm{ml}$ of cadmium) for $24 \mathrm{~h}$. Intracellular $\mathrm{Ca}^{2+}$ is indicated with red fluorescence. (D) The total area of red fluorescence (pixels squared: $\mathrm{px}^{2}$ ) was analysed with image analysis software using three micrographs for each sample. Each data point represents the mean \pm standard deviation. The asterisks $\left(^{*}\right)$ indicate statistically significant differences compared with the PBS-treated control $(\mathrm{p}<0.05)$. The number $(\#)$ sign indicates statistically significant difference compared to the CdTe-QD-treated group $(\mathrm{p}<0.05)$. 81

Figure 3.6. Effects of CdTe-QDs on cellular respiration expressed in rates of $\mathrm{O}_{2}$ consumption from isolated mitochondria. (A) State 2 and state 3 respiration performed with Complex I substrate, glutamate/malate. Rotenone, a Complex I inhibitor, was used as the positive control. (B) State 2 and state 3 respiration performed with Complexes II substrate, succinate. Malonate, a Complex II inhibitor, was used as the positive control. Data points represent the means of three independent experiments \pm standard deviations. The asterisks $\left(^{*}\right)$ indicate statistically significant differences compared to control $(\mathrm{p}<0.05)$ 83

Figure 3.7. Effects of CdTe-QDs on ATP content in HepG2 cells. Cells were treated with CdTe-QDs and $\mathrm{CdCl}_{2}$, at an equivalent concentration of cadmium $(1 \mu \mathrm{g} / \mathrm{ml})$, for 24 h. Data points represent the means of three independent experiments \pm standard deviations. The asterisks $(*)$ indicate statistically significant differences compared to control $(\mathrm{p}<0.05)$. The number $(\#)$ sign indicates statistically significant difference compared to the CdTe-QD-treated group ( $<<0.05)$. 84

Figure 3.8. Effects of CdTe-QDs on mitochondrial ETC complexes. (A) Changes in ETC complex concentrations. (B) Changes in ETC complex activities. Cells were 
treated with CdTe-QDs and $\mathrm{CdCl}_{2}$, at an equivalent concentration of cadmium $(1 \mu \mathrm{g} / \mathrm{ml})$, for $24 \mathrm{~h}$. Data points represent the means of three independent experiments \pm standard deviations. The asterisks $(*)$ indicate statistically significant differences compared to control $(\mathrm{p}<0.05)$ 86

Figure 3.9. Effects of CdTe-QDs on mitochondrial biogenesis expressed as changes in PGC-1 $\alpha$ levels. Cells were treated with CdTe-QDs and $\mathrm{CdCl}_{2}$, at an equivalent concentration of cadmium $(1 \mu \mathrm{g} / \mathrm{ml})$, for $24 \mathrm{~h}$. Data points represent the means of three independent experiments \pm standard deviations. The asterisks $(*)$ indicate statistically significant differences compared to control $(\mathrm{p}<0.05)$. The number $(\#)$ sign indicates statistically significant difference compared to the CdTe-QD-treated group $(\mathrm{p}<0.05) \ldots 87$

\section{Chapter 4}

Figure 4.1. The animal exposure and necropsy scheme used in in vivo studies described in Chapter 4 and 5 of this thesis 102

Figure 4.2. Characterization of CdTe-QDs in water and saline. (A) TEM analysis of diluted CdTe $(1: 1,000)$ deposited on formvar-coated copper grids. (B) DLS analysis of diluted CdTe-QDs (1:1,000). (C) Fluorescence spectra of CdTe-QDs at $125 \mu \mathrm{g} / \mathrm{ml}$ using excitation (Ex.) of $340 \mathrm{~nm}$. (D) Fluorescence standard curves of CdTe-QDs (from 0 $\mu \mathrm{g} / \mathrm{ml}$ to $250 \mu \mathrm{g} / \mathrm{ml}$ ) using excitation (Ex.) of $340 \mathrm{~nm}$ and emission (Em.) of $550 \mathrm{~nm} .108$

Figure 4.3. Detection of CdTe-QDs in tissue homogenates using spectrofluorometry. The fluorescence intensity was measured at Ex. of $340 \mathrm{~nm}$ and Em. of $550 \mathrm{~nm}$. Mice were treated with $10 \mathrm{mg} / \mathrm{kg}$ bw of CdTe-QDs for $24 \mathrm{~h}$. The asterisks (*) indicate statistically significant differences compared to the control group $(\mathrm{p}<0.05)$. 
Figure 4.4. Detection of CdTe-QDs in blood smears and tissue sections $24 \mathrm{~h}$ after mice were treated with CdTe-QDs $(10 \mathrm{mg} / \mathrm{kg}$ bw), using confocal microscopy (Ex. $340 \mathrm{~nm}$, Em. 515/530 nm). (A) blood, (B) lung, (C) heart, (D) liver, (E) spleen, (F) kidney, (G) brain, $(\mathbf{H})$ testis. The blood smears and tissue sections exhibited a green background and CdTe-QDs appeared as brighter green dots as indicated by the arrows. 112

Figure 4.5. Tissue histological images of the livers stained with H\&E, obtained from mice treated with saline (A and E), 6mg/kg bw CdTe-QDs (B and F), $10 \mathrm{mg} / \mathrm{kg}$ bw CdTe-QDs (C and $\mathbf{G})$, and $1.95 \mathrm{mg} / \mathrm{kg}$ bw $\mathrm{CdCl}_{2}$ (D and $\left.\mathbf{H}\right)$. The H\&E stained sections were examined with a light microscopy at magnification of 10x $(\mathbf{A}, \mathbf{B}, \mathbf{C}$, and $\mathbf{D})$ and $60 \mathrm{x}$ $(\mathbf{E}, \mathbf{F}, \mathbf{G}$, and $\mathbf{H})$. The arrows indicate the areas of hemorrhage, apoptotic and necrotic events. 114

Figure 4.6. Tissue histological images of the spleen stained with H\&E, obtained from mice treated with saline (A and $\mathbf{E}), 6 \mathrm{mg} / \mathrm{kg}$ bw CdTe-QDs (B and $\mathbf{F}$ ), $10 \mathrm{mg} / \mathrm{kg}$ bw CdTe-QDs $(\mathbf{C}$ and $\mathbf{G})$, and $1.95 \mathrm{mg} / \mathrm{kg}$ bw $\mathrm{CdCl}_{2}$ (D and $\left.\mathbf{H}\right)$. The H\&E stained sections were examined with a light microscopy at magnification of 20x (A, B, C, and D) and 40x $(\mathbf{E}, \mathbf{F}, \mathbf{G}$, and $\mathbf{H})$. The arrows indicate the areas of hemorrhage and necrotic events.... 115 Figure 4.7. Biochemistry resuls for (A) aspartase transaminase (AST), (B) alanine aminotransferase (ALT), (C) total bilirubin, (D) albumin, and (E) creatinine in serum samples from the control or saline-, $\mathrm{CdTe}-\mathrm{QD}-$ and $\mathrm{CdCl}_{2}$ - treated mice. Serum samples were obtained at $24 \mathrm{~h}$ after mice were treated with different concentrations of CdTe-QDs and $1.95 \mathrm{mg} / \mathrm{kg}$ bw $\mathrm{CdCl}_{2}$, which contains an equivalent cadmium concentration to 6 $\mathrm{mg} / \mathrm{kg}$ bw CdTe-QDs. The asterisks (*) indicates statistically significant differences 
compared to the control $(\mathrm{p}<0.05)$. The number $(\#)$ sign indicates statistically significant difference compared to the CdTe-QD-treated group ( $\mathrm{p}<0.05$ ). 116

Figure 4.8. Haematology analysis of saline-, $\mathrm{CdTe}-\mathrm{QD}$ - and $\mathrm{CdCl}_{2}$-treated mice. (A) White blood counts (WBC), (B) percentage of monocytes, (C) percentage of neutrophils, (D) platelet counts (PLT) at $24 \mathrm{~h}$ after mice were treated with saline, different concentrations of CdTe-QDs, and $1.95 \mathrm{mg} / \mathrm{kg}$ bw of $\mathrm{CdCl}_{2}$ (containing an equivalent of cadmium concentration to $6 \mathrm{mg} / \mathrm{kg}$ bw of CdTe-QDs). (E) Platelets (PLT) counts in mice treated with $5 \mathrm{mg} / \mathrm{kg}$ bw CdTe-QDs for different post-exposure sampling times. The asterisks $(*)$ indicates statistically significant differences compared to the control $(\mathrm{p}<0.05)$. (F) The blood smears were stained with Wright's stain. Mice were treated with $10 \mathrm{mg} / \mathrm{kg}$ bw CdTe-QDs for $24 \mathrm{~h}$. Reticulocytes are indicated by the arrow........ 118

Figure 4.9. Effects of $\mathrm{CdTe}-\mathrm{QDs}$ and $\mathrm{CdCl}_{2}$ on serum amyloid A (SAA). (A) SAA levels $24 \mathrm{~h}$ after mice were treated with saline, different concentrations of CdTe-QDs, and $1.95 \mathrm{mg} / \mathrm{kg}$ bw of $\mathrm{CdCl}_{2}$, which contains the equivalent cadmium concentration as that of $6 \mathrm{mg} / \mathrm{kg}$ bw CdTe-QDs. (B) SAA levels in mice treated with $5 \mathrm{mg} / \mathrm{kg}$ bw CdTeQDs for different time points. The asterisks $\left(^{*}\right)$ indicates statistically significant differences compared to the control $(\mathrm{p}<0.05)$. The number $(\#)$ sign indicates statistically significant difference compared to the $6 \mathrm{mg} / \mathrm{kg}$ bw CdTe-QD-treated group $(\mathrm{p}<0.05) .120$ Figure 4.10. Effects of CdTe-QDs and $\mathrm{CdCl}_{2}$ on selected serum cytokines. Levels of (A) IL-6 and KC, (B) IL-12 (p70) and TNF- $\alpha$ in mice treated with saline, different concentrations of CdTe-QDs, and $1.95 \mathrm{mg} / \mathrm{kg}$ bw of $\mathrm{CdCl}_{2}$, which contains an equivalent cadmium concentration to $6 \mathrm{mg} / \mathrm{kg}$ bw CdTe-QDs, for $24 \mathrm{~h}$. Levels of (C) IL-6 and KC, (D) IL-12 (p70) and TNF- $\alpha$ in mice treated with $5 \mathrm{mg} / \mathrm{kg}$ bw CdTe-QDs for different 
time points. The asterisks $(*)$ indicates statistically significant differences compared to the control $(\mathrm{p}<0.05)$. The number $(\#)$ sign indicates statistically significant difference compared to the CdTe-QD-treated group $(\mathrm{p}<0.05)$.

\section{Chapter 5}

Figure 5.1. Effects of $\mathrm{CdTe}-\mathrm{QDs}$ and $\mathrm{CdCl}_{2}$ on biomarkers of oxidative stress. (A) Total GSH level in the liver at $24 \mathrm{~h}$ post-exposure, (B) total GSH level in the liver at different post-exposure sampling times, $(\mathbf{C})$ total SOD activities in the liver at $24 \mathrm{~h}$, and (D) total SOD activity in the liver at different post-exposure sampling times. (A) and (C) Mice were treated with different concentrations of CdTe-QDs and $1.95 \mathrm{mg} / \mathrm{kg} \mathrm{bw} \mathrm{CdCl}_{2}$ which contains an equivalent cadmium concentration to $6 \mathrm{mg} / \mathrm{kg}$ bw CdTe-QDs for $24 \mathrm{~h}$. (B) and (D) Mice were treated with $5 \mathrm{mg} / \mathrm{kg}$ bw CdTe-QDs and biomarkers were measured at different post-exposure sampling times. The asterisks $(*)$ indicate statistically significant differences compared to the control $(\mathrm{p}<0.05)$.

Figure 5.2. Effects of CdTe-QDs and $\mathrm{CdCl}_{2}$ on antioxidant enzymes. (A) GST activity in the liver (B) CAT activity in the liver. Mice were treated with different concentrations of CdTe-QDs and $1.95 \mathrm{mg} / \mathrm{kg}$ bw $\mathrm{CdCl}_{2}$, which contains an equivalent cadmium concentration to $6 \mathrm{mg} / \mathrm{kg}$ bw CdTe-QDs, for $24 \mathrm{~h}$. The asterisks (*) indicates statistically significant differences compared to the control $(\mathrm{p}<0.05)$.

Figure 5.3. Alteration of oxidative stress gene expression caused by CdTe-QDs and $\mathrm{CdCl}_{2}$. Mice were treated with different concentrations of CdTe-QDs and $1.95 \mathrm{mg} / \mathrm{kg}$ bw $\mathrm{CdCl}_{2}$, which contains an equivalent cadmium concentration to $6 \mathrm{mg} / \mathrm{kg}$ bw CdTe-QDs, for $24 \mathrm{~h}$. Cat $=$ catalase, $\mathrm{Gpx}=$ glutathione peroxidase, Gstk $=$ glutathione S-transferase 
kapa, Gstp $=$ glutathione S-transferase pi, Hmox $=$ heme oxygenase, $\mathrm{Ncf}=$ neutrophil cytosolic factor, $\operatorname{Prdx}=$ peroxiredoxin, Sod $=$ superoxide dismutase. $(+)=$ mRNA up regulation, $(-)=$ mRNA down-regulation.

Figure 5.4. Effects of CdTe-QDs and $\mathrm{CdCl}_{2}$ on the apoptosis biomarkers. (A) Active caspase-3 level and (B) cleaved PARP level. Data were represented by fluorescence intensities (FI). Mice were treated with different concentrations of CdTe-QDs and 1.95 $\mathrm{mg} / \mathrm{kg}$ bw $\mathrm{CdCl}_{2}$, which contains an equivalent cadmium concentration to $6 \mathrm{mg} / \mathrm{kg}$ bw CdTe-QDs, for $24 \mathrm{~h}$. The asterisks $\left(^{*}\right)$ indicates statistically significant differences compared to the control $(\mathrm{p}<0.05)$. The number (\#) sign indicates statistically significant differences of $\mathrm{CdCl}_{2}$ compared to the CdTe-QD-treated group ( $\left.\mathrm{p}<0.05\right)$. 145

Figure 5.5. Alteration of apoptotic gene expression caused by $\mathrm{CdTe}-\mathrm{QDs}$ and $\mathrm{CdCl}_{2}$. Mice were treated with different concentrations of CdTe-QDs and $1.95 \mathrm{mg} / \mathrm{kg}$ bw $\mathrm{CdCl}_{2}$, which contains an equivalent cadmium concentration to $6 \mathrm{mg} / \mathrm{kg}$ bw CdTe-QDs for $24 \mathrm{~h}$.

Figure 5.6. Changes in liver hepatocyte mitochondrial morphology, structure, and number induced by CdTe-QDs as examined by TEM. Mice were treated with different concentrations of CdTe-QDs and $1.95 \mathrm{mg} / \mathrm{kg}$ bw $\mathrm{CdCl}_{2}$, which contains an equivalent cadmium concentration to $6 \mathrm{mg} / \mathrm{kg}$ bw CdTe-QDs, for $24 \mathrm{~h}$.

Figure 5.7. Effects of CdTe-QDs and $\mathrm{CdCl}_{2}$ on the mitochondrial ETC complexes. (A) Alteration of the ETC complexes at the protein level. The asterisks $\left(^{*}\right)$ indicates statistically significant differences compared to the control $(\mathrm{p}<0.05)$. (B) Changes in ETC complex gene expression. Mice were treated with different concentrations of CdTeQDs and $1.95 \mathrm{mg} / \mathrm{kg}$ bw $\mathrm{CdCl}_{2}$, which contains an equivalent cadmium concentration to 
$6 \mathrm{mg} / \mathrm{kg} / \mathrm{ml} \mathrm{CdTe-QDs}$, for $24 \mathrm{~h}$. Ndufa = NADH dehydrogenase Complex I subunit; Sdhd = Succinate dehydrogenase, Complex II, subunit D; Uqcr = Ubiquinol-cytochrome c reductase, Complex III, subunit; Cox $=$ Cytochrome c Oxidase, Complex IV, subunit; Atp $=$ ATP synthase, complex V, subunit. $(+)=$ mRNA up regulation, $(-)=$ mRNA down-regulation. 148

Figure 5.8. Effect of CdTe-QDs and $\mathrm{CdCl}_{2}$ on the ATP level. (A) Mice were treated with different concentrations of CdTe-QDs and $1.95 \mathrm{mg} / \mathrm{kg}$ bw $\mathrm{CdCl}_{2}$, which contains an equivalent cadmium concentration to $6 \mathrm{mg} / \mathrm{kg}$ bw CdTe-QDs for $24 \mathrm{~h}$. (B) Mice were treated with $5 \mathrm{mg} / \mathrm{kg}$ bw CdTe-QDs for different time points. The asterisks $\left(^{*}\right)$ indicates statistically significant differences compared to the control $(\mathrm{p}<0.05)$. The number $(\#)$ sign indicates statistically significant difference compared to the CdTe-QD-treated group $(\mathrm{p}<0.05)$ 150

Figure 5.9. Effect of $\mathrm{CdTe}-\mathrm{QDs}$ and $\mathrm{CdCl}_{2}$ on mitochondrial biogenesis biomarker, PGC-1 $\alpha$ in the liver. (A) Mice were treated with different concentrations of CdTe-QDs and $1.95 \mathrm{mg} / \mathrm{kg}$ bw $\mathrm{CdCl}_{2}$, which contains an equivalent cadmium concentration to 6 $\mathrm{mg} / \mathrm{kg}$ bw CdTe-QDs for $24 \mathrm{~h}$. (B) Mice were treated with $5 \mathrm{mg} / \mathrm{kg}$ bw CdTe-QDs for different time points. The asterisks $(*)$ indicates statistically significant differences compared to the control $(\mathrm{p}<0.05)$. The number $(\#)$ sign indicates statistically significant difference compared to the CdTe-QD-treated group $(\mathrm{p}<0.05)$. 151

\section{Chapter 6}

Figure 6.1. Proposed model that highlights mitochondrial effects of CdTe-QDs as the mechanisms of hepatotoxicity induced by these NPs. (1) CdTe QDs interact with the 
ETC leading to (2) increased ROS generation from Complex I and III, and thus (3) oxidative stress leading to redox signaling, further mitochondrial dysfunction, and apoptosis in hepatocytes. Oxidative stress results in (4) cytochrome c (cyt c) release and (5) mitochondrial pearmeability transition pore (PTP) opening, leading to apoptosis. PTP opening and disruption of mitochondrial membrane potential cause (6) the release of calcium $\left(\mathrm{Ca}^{2+}\right)$ into the cytosol leading to increased intracellular $\mathrm{Ca}^{2+}$ level. (7) Cadmium $\left(\mathrm{Cd}^{2+}\right)$ released from CdTe-QDs can also interact with the ETC resulting in similar effects on mitochondria. (8) Both intact CdTe-QDs and $\mathrm{Cd}^{2+}$ can also induce ROS production within cells or mitochondria via redox activity, leading to redox signaling, mitochondrial dysfunction and apoptosis in cells. 170 


\section{List of Abbreviations}

\begin{tabular}{|c|c|}
\hline ADP & Adenosine diphosphate \\
\hline AFM & Atomic force microscopy \\
\hline ALT & Alanine transaminase \\
\hline AST & Aspartate aminotransferase \\
\hline ATP & Adenosine triphosphate \\
\hline $\mathrm{Bcl} 2$ & B-cell lymphoma 2 \\
\hline Bax & Bcl-2-like protein 4 \\
\hline Bid & $\mathrm{BH} 3$-only protein \\
\hline $\mathrm{Bw}$ & Body weight \\
\hline CAT & Catalase \\
\hline CCCP & Carbonylcyanide m-chlorophenylhydrazone \\
\hline $\mathrm{CdCl}_{2}$ & Cadmium chloride \\
\hline $\mathrm{CdS}$ & Cadmium sulfide \\
\hline $\mathrm{CdSe}$ & Cadmium selenide \\
\hline $\mathrm{CdTe}$ & Cadmium telluride \\
\hline CNT & Carbon nanotube \\
\hline $\mathrm{COOH}$ & Carboxyl group \\
\hline Cox & Cytochrome c Oxidase, Complex IV, subunit \\
\hline Cyt c & Cytochrome c \\
\hline DHE & Dihydroethidium \\
\hline DLS & Dynamic light scattering \\
\hline
\end{tabular}




\begin{tabular}{|c|c|}
\hline DMEM & Dulbecco's Modified Eagle Medium \\
\hline DMSO & Dimethyl sulfoxide \\
\hline DTNB & 5, 5'-Dithio-bis(2-nitrobenzoic acid) \\
\hline DNA & Deoxyribose nucleic acid \\
\hline$\Delta \Psi \mathrm{m}$ & Membrane potential \\
\hline EDTA & Ethylenediaminetetraacetic acid \\
\hline EGTA & Ethylene glycol tetraacetic acid \\
\hline ELISA & Enzyme-linked immunosorbent assay \\
\hline EMEM & Eagle's minimum essential medium \\
\hline EIA & Enzyme Immunometric Assay \\
\hline Em & Emission \\
\hline Erk1/2 & Extracellular signal-regulated kinases \\
\hline ETC & Electron transport chain \\
\hline Ex & Excitation \\
\hline IV & Intravenous \\
\hline $\mathrm{FADH}_{2}$ & Reduced flavin adenine dinucleotide \\
\hline FBS & Fetal bovine serum \\
\hline FI & Fluorescence intensity \\
\hline GI & Gastrointestinal \\
\hline Gpx & Glutathione peroxidase \\
\hline GSH & Reduced glutathione \\
\hline GSSG & Oxidized glutathione \\
\hline GST & Glutathione-S-transferase \\
\hline
\end{tabular}




$\begin{array}{ll}\text { Gstk } & \text { Glutathione-S-transferase kappa } \\ \text { Gstp } & \text { Glutathione-S-transferase pi } \\ \mathrm{H}_{2} \mathrm{O}_{2} & \text { Hydrogen peroxide } \\ \mathrm{H} \& \mathrm{E} & \text { Hematoxylin and eosin } \\ \text { HepG2 } & \text { Human hepatocellular carcinoma } \\ \text { HEPES } & \text { 4-2-Hydroxyethyl)-1-piperazineethanesulfonic } \\ \text { Hmox } & \text { Heme oxygenase } \\ \text { HRP } & \text { Horseradish peroxidase } \\ \text { ICP-OES } & \text { Inductively coupled plasma optical emission spectrometry } \\ \text { ICP-MS } & \text { Inductively coupled plasma mass spectrometry } \\ \text { IETD-pNA } & \text { Acetyl-Ile-Glu-Thr-Asp p-nitroaniline } \\ \text { IL-6 } & \text { Interleukin 6 } \\ \text { IL-12(p70) } & \text { Interleukin 12p70 } \\ \text { JNK } & \text { c-Jun N-terminal kinases } \\ \text { KC } & \text { Keratinocyte chemoattractant } \\ \text { LC/MS } & \text { Liquid chromatography/mass spectrometry } \\ \text { LED } & \text { Light emitting devices } \\ \text { MAPK } & \text { Mitogen-activated protein kinases } \\ \text { mRNA } & \text { Messenger ribonucleic acid } \\ \text { MOPS } & \text { Mercaptopropionic acid } \\ \text { MPT } & \text { Mitochondrial permeability transition } \\ \text { MPA } & \text { Hethylthiazol-2-yl)-2,5-diphenyl tetrazolium bromide }\end{array}$




\begin{tabular}{|c|c|}
\hline mtDNA & Mitochondrial deoxyribonucleic acid \\
\hline $\mathrm{NaCl}$ & Sodium chloride \\
\hline $\mathrm{NaCN}$ & Sodium cyanide \\
\hline NADH & Nicotinamide adenine dinucleotide \\
\hline NADPH & Reduced nicotinamide adenine dinucleotide phosphate \\
\hline $\mathrm{Na}_{2} \mathrm{TeO}_{3}$ & Sodium tellurite \\
\hline Ncf & Neutrophil cytosol factor \\
\hline Ndufa & NADH dehydrogenase ubiquinone, Complex I, subunit 1-alpha \\
\hline $\mathrm{NH}_{2}$ & Amine group \\
\hline NP-40 & Nonyl phenoxypolyethoxylethanol \\
\hline NM & Nanomaterial \\
\hline Nrf2 & NF-E2-related Factor 2 \\
\hline NP & Nanoparticle \\
\hline${ }^{1} \mathrm{O}_{2}$ & Singlet oxygen \\
\hline $\mathrm{O}_{2}^{-}$ & Superoxide anion \\
\hline $\mathrm{OH}$ & Hydroxyl radicals \\
\hline OXPHOS & Oxidative phosphorylation \\
\hline PARP & Poly ADP-ribose polymerase \\
\hline PBS & Phosphate buffered saline \\
\hline $\mathrm{PE}$ & Phycoerythrin \\
\hline PGC-1 $\alpha$ & Peroxisome proliferator-activated receptor- $\gamma$ coactivator 1 -alpha \\
\hline PLT & Platelet \\
\hline Prdx & Peroxiredoxin \\
\hline
\end{tabular}




\begin{tabular}{|c|c|}
\hline PS & Phosphatidylserine \\
\hline PTP & Permeability transition pore \\
\hline ROS & Reactive oxygen species \\
\hline RT & Room Temperature \\
\hline SAA & Serum amyloid $\mathrm{A}$ \\
\hline Sdhd & Succinate dehydrogenase, Complex II, subunit D \\
\hline SOD & Superoxide dismutase \\
\hline STS & Staurosporine \\
\hline TCA & Tricarboxylic acid cycle \\
\hline TEM & Transmission electron microscopy \\
\hline TMB & $3,3^{\prime}, 5,5^{\prime}$ - Tetramethylbenzidine \\
\hline TMRE & Tetramethylrhodamine, ethyl ester \\
\hline TNF- $\alpha$ & Tumor necrosis factor alpha \\
\hline Uqcr & Ubiquinol-cytochrome c reductase, Complex III, subunit \\
\hline WBC & White blood cells \\
\hline $\mathrm{ZnS}$ & Zinc sulfide \\
\hline
\end{tabular}




\section{Chapter: Introduction}

\subsection{Nanomaterials and potential health impacts of nanoparticles}

Nanomaterials are materials consisting of, or containing, structures with at least one dimension in their physical size ranging between 1 and $100 \mathrm{~nm}$ ((BSI, 2011; ISO, 2010). Nanomaterials can originate from natural sources (volcanic eruptions, forest fires, hydrothermal vent system, or dust volatilization), be created incidentally (as by-product of industrial processes and combustion engines), or be engineered for a specific application (Farre et al., 2011). Nanomaterials composed of engineered nanoparticles (NPs) possess unique physico-chemical characteristics leading to advanced magnetic, electrical, mechanical, optical, thermal, and structural properties compared to the same material of a larger size (bulk material) (Nel et al. 2006). The special characteristics of NPs are based on two features. The first feature is the increased surface to volume ratio which results in a higher proportion of atoms at the surface and an increase in chemical reactivity of the materials. The second feature of the nanoscale is the dominance of physical quantum effects which influences properties like transparency or conductivity (Nel et al. 2006). These special properties make NPs attractive for exploitation in variety of fields including medicine, cosmetics, electronics, textiles, engineering, and consumer products. Some of the common classes of engineered NPs include: (1) carbon-based materials such as fullerenes, carbon nanotubes, carbon black, (2) metal-based materials such as nanogold, nanosilver, metal oxides, quantum dots (QDs), and (3) others including dendrimers, nanoclays, polystyrene, silicon oxide, etc. (Nowack and Bucheli, 2007).

The field of nanotechnology is expanding at a rapid rate, resulting in increased production and use of NPs each year. It is suggested that the number of nanotechnology 
products will double every three years, achieving a 3 trillion US\$ global market by 2020 (Roco, 2011). As the use of NPs is rapidly growing, there is increasing concern that these materials might pose potential risks to human health (Dreher, 2004). Increased NP application and production will inevitably lead to increased incidence of the materials in the environment and to increasing exposure of humans both intentionally and unintentionally. The possible portals where NPs may enter the human body are the lungs via inhalation, the gastrointestinal tract via digestion, the skin, and the blood vessels via intravenous injection (IV) from diagnostic and therapeutic applications (Oberdorster et al. 2005). Inhalation represents an important route of human exposure to NPs as airborne NPs present in polluted air, occupational setting, and house-hold air. Through inhalation, NPs can easily enter into the nasal and tracheal/bronchial regions of the respiratory tract. Studies have also shown that NPs are capable of penetrating the alveoli deep in the lung (Oberdorster et al., 2005). Another potential route of human exposure to NPs is oral exposure through gastrointestinal tract. Based on their uses in food, food packaging, drug delivery, and cosmetics, NPs have the potential to be ingested and pass through to the GI tract (Oberdorster et al. 2005). Multiple parameters such as size, surface charge, attachment to ligand or type of ligand could control their targeting and absorption in GI tract (Hillyer and Albrecht, 2001). Another important route of human exposure to NPs is dermal exposure. With their use in cosmetics, suncreens, band aids, clothing, and so on, the incidence of dermal NP exposure is rapidly increasing (Liang et al., 2013). Multiple parameters such as dose, vehicle, valence, age and origin of the skin are involved in the skin absorption process (Baroli, 2010). Exposure to NPs via IV occurs when NPs are used for biomedical applications such as diagnostic tools or drug carriers (Stern and 
McNeil, 2007). Intravenous administration, which often is targeted at a specific organ or tissue, results in direct contact of NPs with blood, upon which the particle surface becomes coated with numerous proteins that can enhance recognition and uptake by cells of the reticuloendothelial system (RES) (Aggarwal et al. 2009; Lacerda et al. 2010). Intravenous administration of NPs could result in potential risk of tissue and systemic bioavailability of NPs, leading to organ and systemic toxicity, and needs to be addressed for their safety assessment (Hahn et al. 2011; Irache et al. 2011).

While their unique physico-chemical properties make NPs attractive for new product development providing benefit to society, these properties are also thought to be responsible for the toxicity associated with NPs (Nel et al. 2006). Studies on nanotoxiccology have shown that both physical and chemical factors, including size, shape, surface area, surface chemistry, surface charge, agglomeration state, crystal structure and chemical composition, are important determinants of NP toxicity (Sajid et al., 2015). NP size has been shown to be one the most important properties that influence NP behaviour in biological systems and thus influence their toxicity (Donaldson et al 2004). As NP size decreases, the surface to volume ratio increases exponentially, which make these particles more reactive and toxic. Owing to their small size, NPs can transport across cell membranes or be taken up into target cells, causing changes in subcellular structures and affecting cellular activity (Nel et al, 2006). Similarly, it has been shown that within a biological system, at their nanoscale, NPs are capable of distributing throughout the body and penetrating many physiological barriers to reach vital organs once translocated to the blood circulation (Sajid et al., 2015). Chemical composition and the intrinsic toxicological properties of the chemical have also been shown as important 
determinants to the biological effects of NPs as these factors determine reactivity of NPs (Donaldson et al 2004). Reactive NPs can induce catalytic reactions within the cells and result in production of free radical species within biological systems. This unusual reactivity makes NPs highly toxic (Stark 2011). Surface chemistry (ie. reactive groups on a particle surface) is also another important property that affects NP toxicity as this property defines charge and reactivity of NPs (Lai, 2012). In NP structure, different functionalities create different charges over NP surfaces. For examples, carboxylic acid $(-\mathrm{COOH})$ functionalized NPs are considered negatively charged while amine $\left(-\mathrm{NH}_{2}\right)$ functionalized NPs are considered positively charged. Surface chemistry, coating, and modification have been shown to influence NP behavior and distribution in biological systems as charge on NPs makes them more or less reactive towards cells and proteins (Lai, 2012). NPs are known to have various shapes such as spherical, needle-like, tubes, platelets, etc., and these shapes may have effects on the kinetics of deposition and absorption to the body (Sajid et al., 2015). Similarly, NP aggregates and agglomerates forms, resulting in different size, shape, deposition, and clearance kinetics, have been reported to be associated with differential cellular responses as well as organ distribution and effects (Shvedova et al., 2005).

Since the past decade, there has been a growing body of evidence showing toxicity of NPs in both cell cultures and animal models (Boczkowski and Hoet, 2010). In addition, the existing nanotoxicology literature suggests significant toxicity associated with many of NPs currently in use (Xia et al., 2009). For examples, studies have shown that carbon nanotubes (CNTs) caused oxidative stress and inflammation in cell lines, raising concerns about the genotoxic and carcinogenic potential of CNTs. Animal studies 
have shown that pulmonary exposures to CNT consistently generate an asbestos-like toxicological response characterised by persistent inflammation, granulomas and fibrosis with low no-effect levels (Aschberger et al., 2010). In vitro and in vivo toxicity studies in mammalian species show that silver NPs have the capability to enter cells and cause cellular damage to the skin, liver, lung, brain, vascular system, and reproductive organs (Wijnhoven, 2009). Similarly, acute and chronic exposure to zinc oxide NPs were shown to cause inflammation in animal models (Osmond, 2010). Treatment of titanium dioxide NPs to cells and rodents also caused cytotoxicity, oxidative stress, and pulmonary inflammation (Shi et al., 2013). In spite of the vast number of studies in the past decades, there is still a great gap in nanomaterial toxicology as the precise information in the mechanisms of NP toxicity is lacking.

\subsection{What are quantum dots}

Quantum dots (QDs) are engineered NPs which are also known as fluorescent semiconductor nanocrystals (Bruchez et al., 1998). QDs are approximately 2-100 nm, containing about 200-10,000 atoms (Smith et al., 2008; Juzenas et al., 2008). Structurally, QDs are composed of a metal core which determines their color, an inorganic shell which helps to enhance stability and brightness, and a polymer layer or functional group which enhances water solubility and conjugation capacity (Michalet et al., 2005). QD cores are typically made of a variety of metal complexes such as semiconductors, noble metals, and magnetic transition metals (Yin and Alivisatos, 2005). Traditionally, QD cores are comprised of group II-VI series elements. QDs that are created from this group include cadmium selenium (CdSe), cadmium tellurium (CdTe), 
zinc sulfide $(\mathrm{ZnS})$, and zinc selenium $(\mathrm{ZnSe})$. Another group of QDs that are created from III-V series elements are gallium arsenate (GaAs), gallium nitride $(\mathrm{GaN})$, indium phosphate (InP), and indium arsenate (InAs) (Gerion et al., 2001; Kim et al., 2003). The cores of QDs can be covered with a shell that is commonly made of $\mathrm{ZnS}, \mathrm{ZnSe}, \mathrm{CdS}$, or InP to increase the stability and to maintain the quantum yield of QDs (Wang et al., 2009). QD shells are further covered with polymers which can act as an interface for enhanced water solubility and for functionalization with biomolecules making these NPs suitable for biological and medical applications (Michalet et al., 2005).

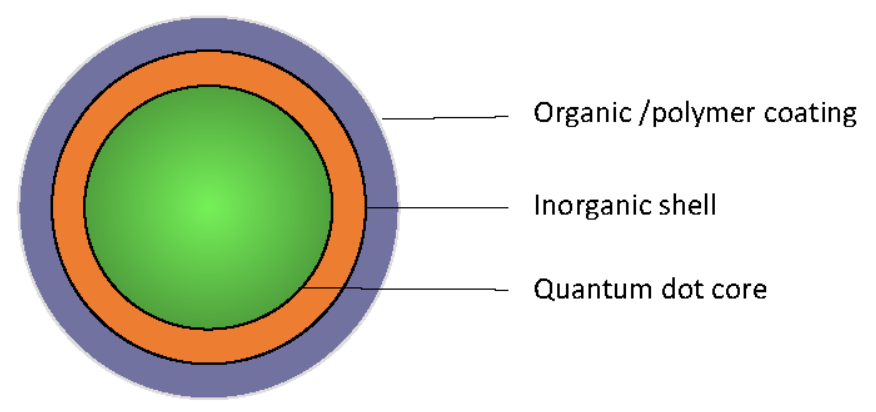

Figure 1.1. Basic structure of a core-shell QD and a core-shell QD conjugated with biofunctional molecules,

QDs possess unique optical and electronic properties, including tunable emission wavelength, broadband absorption spectrum, and photostability (De Wild et al, 2003). Specifically, QDs have discrete energy levels that are specific to material composition (Alivisatos, 1996). The boundaries of these energy levels can be precisely tuned by the addition or subtraction of certain atoms in QDs. This quantum confinement effect 
provides QDs narrow emission spectra and tunable emission wavelength, which can be manipulated by changing their core size and composition (Michalet et al., 2005). More importantly, the QDs can be tuned to emit emission ranging from UV to near-infrared region (Smith et al., 2006). QDs have broad absorption spectra, allowing them to be excited by light of a wide range of wavelengths (Michalet et al., 2005). Thus, QDs can be simultaneously excited QDs with different emission spectra for multiplex imaging using a single excitation source (Mukundan et al., 2010). The high photostability is another unique property of QDs that provides them stability and capacity of withstanding many cycles of excitation for long periods of time with a high level of brightness (Michalet et al., 2005). These unique properties of QDs make them useful in applications in variety of fields.

\subsection{Application of quantum dots}

Owing to their unique optical and electrical properties, QDs have been extensively used in microelectronics and biomedical and biological research (De Wild et al., 2003). In the past few decades, QDs have been used in a number of commercial applications including optoelectronics, quantum computing, solar cells, light emitting devices, and security inks (Harrison et al., 2000; Wu et al., 2004; Zhao et al., 2006; Hetsch et al., 2011; Qian et al., 2011). In addition, cadmium-based QDs such as CdTeQDs and CdSe-QDs have gained much interest as emerging fluorophores with great potential in therapeutic targeting and in medical and molecular imaging (Chan et al., 2002; Gao et al., 2004; Scherer et al., 2002). Since 2002, there has been $300 \%$ increase in the number of publications related to biomedical applications of cadmium based QDs 
(Medintz et al., 2008; Zrazhevskiy et al., 2010). Some examples of biomedical applications of QDs include preparation of antibody-conjugated polymeric micelle coated $\mathrm{CdSe} / \mathrm{ZnS}$ QDs for targeting and imaging of human prostate cancer growing in nude mice (Gao et al., 2004); development of arginine-glycine-aspartic acid (RGD) peptide-labeled $\mathrm{CdTe} / \mathrm{ZnS}$ quantum dots (QDs) for in vivo targeting and imaging of tumor vasculature (Cai et al., 2006); synthesis of bioconjugated $\mathrm{CdSe} / \mathrm{CdS} / \mathrm{ZnS}$ quantum rods and $\mathrm{CdTe} / \mathrm{CdS}$ quantum dots to be used as ultrasensitive optical nanoprobes for tumor vasculature imaging in live animals (Yong et al., 2009; Yong et al., 2010); the preparation of peptide-conjugated phospholipid-micelle-encapsulated silicon quantum dots for in vivo tumor imaging (Erogbogbo et al., 2010); or the integration of drug molecules with bioconjugated QDs for traceable drug delivery and therapy in vitro and in vivo (Yong et al., 2012). All these studies have laid an important foundation in engineering of QDs for disease therapy research and applications. Thus, current and future applications of QDs impact a broad range of industrial markets. According to a recent report titled "Quantum Dot (QD) Market - Global Analysis, Growth, Trends, Opportunities, Size, Share and Forecast through 2020" published by Allied Market Research, a global market research company based in Portland, Oregon, the global quantum dots (QD) market accrued revenue of \$316 million in 2013 and it is expected to grow to $\$ 5,040$ million by 2020 . The volume consumption will grow a much faster rate of $116.5 \%$ during the same period to reach 72 tons in 2020 (Allied Market Research, 2014). While the applications of these QDs are growing and the production of these NPs is rapidly increasing, the toxicity of these NPs is a concern and needs to be thoroughly investigated. 


\subsection{Toxicity of quantum dots}

Similar to other types of NPs, the increased use and production of QDs have resulted in to potential increases in human exposure from occupational, medical and environmental sources, leading to concerns about environmental and health risk of these NPs. In addition, the same characteristics that give QDs unique properties (e.g., nanometer size, heavy metal core, high surface area) also contribute to uncertainty regarding their long-term, potentially deleterious effects on environmental and public health. Such concerns have been a drawback to the introduction of QDs into the clinical setting. Thus, investigation of the toxicity of QDs is critical in determining the fate of this promising nanotechnology.

\subsubsection{In vitro toxicity and proposed mechanisms}

In vitro, toxicity of QDs has been reported in different cell lines and primary cells. Some of these including kidney, liver, immune, skin, neuron, breast cancer, and colon cancer cells (as reviewed by Pelley et al., 2009; Yong et al., 2013). Numerous studies investigating in vitro QD toxicity have concluded that QDs can be cytotoxic, affect immune responses, and cause cell stress (Pelley et al., 2009; Yong et al., 2013). However, the information obtained from in vitro studies suggests that the underlying mechanisms of QD-mediated toxicity in various cell lines are not well understood (Pelley et al., 2009; Yong et al., 2013). Toxicity of cadmium-based QDs was proposed to be associated with (1) leaching of free $\mathrm{Cd}^{2+}$ from the QD core, (2) generation of reactive oxygen species (ROS), or (3) intrinsic properties of QDs such as size and surface reactivity. 
Many studies have suggested that the presence of cadmium in the QD metal core is a primary source of QD toxicity (Derfus et al., 2004; Chang et al., 2006). The release of $\mathrm{Cd}^{2+}$ ions from QDs is hypothesized to result from the degradation or oxidation of the metal core within the specific experimental/environmental conditions, and the amount of free $\mathrm{Cd}^{2+}$ ions in solutions of QDs correlates with QD-induced cytotoxicity (Derfus et al., 2004; Kirchner et al., 2005; Xu et al., 2010). It was subsequently hypothesized that the addition of a shell or a polymer based material to the QD core would reduce the level of oxidation, thus inhibiting QD toxicity (Derfus et al., 2004).

ROS generation has been reported to contribute significantly to QD induced cellular damage (Samia et al., 2006, Lovric et al., 2005a; Lovric et al., 2005b). ROS generation has been considered as one of the common properties of many types of metalbased nanoparticles (NPs) and a major contributor to NP-induced toxicity (Donaldson et al., 2001). ROS generated in cells include singlet oxygen $\left({ }^{1} \mathrm{O}_{2}\right)$, superoxide anion $\left(\mathrm{O}_{2}^{-}\right)$, hydroxyl radicals ( $\mathrm{OH})$ and hydrogen peroxide $\left(\mathrm{H}_{2} \mathrm{O}_{2}\right)$. The exact mechanisms for the production of these reactive species induced by NPs are not well understood. QDs are thought to have the ability to spontaneously induce ROS production because of their electron configuration (Maysinger and Lovric, 2007). ROS generation in cells can result in oxidative stress and affect cellular signaling cascades that control different cellular processes leading to cell damage and triggering apoptosis (Simon et al., 2000). ROS role in QD toxicity was first reported in PC12 cells and this study showed that pre-treatment with antioxidants negated cell death, suggesting that QD toxicity was mediated via ROS production (Lovric et al., 2005a). Another study of the same group also showed ROS produced from mercaptopropionic acid (MPA) coated CdTe QDs led to cytotoxicity in 
human breast cancer cells (MCF-7) and affected cell function (Lovric et al., 2005b). Further research into the ability of QDs to produce ROS and cause oxidative stress was performed on IMR-32 cells and demonstrated that TOPO capped QDs significantly decreased cell metabolic competence by increasing production of ROS (Chan et al., 2006). Most recently, ROS production from different types of QDs were suggested to be the mechanisms of elevated cytoplasmic calcium levels in primary cultures of hippocampal neurons (Tang et al., 2008) and of observed toxic effects in mice (Haque et al., 2013).

Several studies, however, have also suggested that toxicity of CdTe-QDs could arise from several intrinsic properties such as size, chemical composition, and surface coating components (Hardman, 2006). For example, a study that investigated the cytotoxicity of mercaptoundecanoic acid (MUA) CdSe QDs in Vero cells (African green monkey's kidney cells), primary human hepatocytes, and HeLa cells reported that the observed cytotoxicity and cell death was due to the TOPO MUA-SSA cap on the QDs (Shiohara et al., 2004). In another study, CdSe core/ZnS shell QDs of five surface modifications were used for examining the toxic effects on mouse lymphoma EL-4 cells. It was observed that negatively charged QDs were less toxic compared to positively charged QDs, suggesting QD surface chemistry as an indicator of their toxicity (Hoshino et al., 2004). Similarly, examination of the effects of both green and red positively and negatively surface charged 'naked' CdTe QDs in rat neuron-like pheochromocytoma (PC12) and murine microglial (N9) cells revealed that, at the same test concentrations, positively charged QDs induced greater cytotoxicity than the negatively charged QDs in these cells (Lovric et al., 2005b). In the same study, QD size was also demonstrated as a 
determinant of QD toxicity as the authors suggested size-specific QD localization inside the cell. It was reported that smaller green QDs were able to penetrate into the nucleus, possibly causing damage to the cells deoxyribose nucleic acid (DNA) content, and thus attributed as the potential process responsible for the increased cytotoxicity observed (Lovric et al., 2015b). Additionally, other studies on toxicity of polymer coated QDs reported that a significant cytotoxicity in human neuroblastoma IMR-32 and HepG2 cells was caused by QD organic coating but not the QD core (Chan et al., 2006; Guo et al., 2007). Therefore, even though there have been numerous studies that reported the toxicity of QDs in various cell lines, the underlying mechanisms by which these NPs cause cell damage and death are still poorly understood.

\subsubsection{In vivo toxicity}

Currently, information on the in vivo toxicity of cadmium-based QDs is limited. To date, there have been only a few studies reporting toxicity of cadmium-based QDs in invertebrate and aquatic animal models (Gagne et al., 2008; Peyrot et al., 2009; Gagne et al., 2010; Kim et al., 2010; Lee et al., 2010; Pace et al., 2010; King-Heiden et al., 2009, Rocha et al., 2014, Bruneau et al., 2015). Studies on the toxicity of QDs in mussels, rainbow trout, Daphnia, and zebrafish showed that these NPs caused immunotoxicity (Gagne et al., 2008; Gagne et al., 2010; Bruneau et al., 2015) oxidative stress (Gagne et al., 2008), genotoxicity (Rocha et al., 2014), and mortality (Pace et al., 2010; KingHeiden et al., 2009).

For rodent models, in vivo toxicity has also been investigated using intravenous injection (IV), intraperitoneal (IP), dermal exposure, intranasal, intratracheal instillation, 
and inhalation (as reviewed by Hardman, 2006; Pelley et al., 2009; Yong et al., 2013). Among these studies, IV has been most used as it is an important route of QD exposure due to the NP potential diagnostic and therapeutic applications. Injected QDs have been examined for their toxicokinetic and toxicodynamic profiles as well as potential systemic toxicity using traditional toxicological endpoints including hematology, clinical chemistry, and histopathology (Pelley et al., 2009; Yong et al., 2013). In contrast to most in vitro studies and in vivo studies on invertebrate and aquatic animal models, which showed apparent toxic effects of cadmium-based QDs, in vivo studies in rodents have given conflicting information on the toxicity of these NPs. A few studies have shown toxicity of cadmium-based QDs in mice and rats, including changes in locomotor activity (Zhang et al., 2007), effects on immunity (Tang et al., 2013), vascular thrombosis in the lung leading to death (Geys et al., 2008; Tang et al., 2013), and liver, spleen, and kidney injuries (Liu et al., 2011; Tang et al., 2013). By contrast, several studies reported no observable toxicity induced by cadmium-based QDs in rodents (Law et al., 2009; Hauck et al., 2010; Su et al., 2011; Haque et al., 2012). Toxicity of CdTe-QDs was also investigated in non-human primates in a recent study (Ye at al., 2012). The authors of this study reported that at concentrations suitable for imaging techniques, MPA capped CdTe-QDs caused no acute toxicity in these animals.

The discrepancies in the results among toxicity studies on cadmium-based QDs could be explained by the variation in particle size, coating, dose, and exposure conditions that were used in different in vivo studies (Haque et al., 2013). Consequently, despite the effort by many research groups to characterize the behavior and to measure the toxicity of QDs in vivo, little is known on the biological effects of these NPs in 
relevant animal models and in human. Given that there are some discrepancies among previous reports concerning the degree of toxicity, the sources of toxicity, and the underlying mechanisms of toxicity of CdTe-QDs, further research is needed to clarify the potential effects of existing products and more advanced designs of CdTe-QDs on human health and environment.

\subsection{The liver and hepatotoxicity}

The liver is the primary organ involved in the metabolism and detoxification of xenobiotics. The organ plays an important role in many vital functions of the body including lipid and cholesterol metabolism, production of hormones, phagocytosis of debris and bacteria, iron metabolism, production of bile, processing and storage of nutrients and vitamin A, and synthesis of blood proteins including albumin, lipoproteins, transferrin, growth factors and coagulation factors (Dyce et al., 1987).

Traditionally, in vertebrates, including mice, rats, and human, the liver is divided into four lobes (MacSween et al., 2002). Each lobe of the liver contains thousands of equally built lobules, and is served by two distinct blood supplies, the hepatic artery which supplies oxygenated blood and the portal vein which feeds blood from the intestinal system (including the pancreas and the spleen) and is rich in nutrients but is low in oxygen. The blood flows out of the liver via the hepatic vein in the direction of the inferior vena cava (MacSween et al., 2002). The hepatic lobule is define histologically as a hexagonal region consisting of a central vein, plates of hepatocytes arranging radially around the central vein, and four to six surrounding portal triads. The portal triad is made up of a hepatic arteriole, portal vein and bile duct. The majority of blood entering the 
liver is from the intestine, which enters through the portal vein and passes through the sinusoidal space between radially-aligned hepatocytes. Oxygenated blood from the heart also enters the portal triads in this way via the hepatic arterioles. In both cases, the blood filters through the hepatocytes and exits the liver by way of the central veins. At the same time, bile is synthesized within the hepatocytes and flows away from the central vein through canaliculi (MacSween et al., 2002).

The functional unit of the liver is described as the acinus and is divided into three zones: zone one corresponds to periportal region, zone two corresponds to midzonal region, and zone three corresponds to centrilobular region (Treinen-Moslen et al., 2001). These three zones vary in localization as well as functionality. In general, zone one hepatocytes reside in an oxygen and bile salt-rich environment because of their proximity to the portal triad. In this zone, hepatocytes have high expression of cytochrome P450 (CYP450) enzymes, important contributors to phase I drug metabolism. By contrast, zone three hepatocytes reside in an oxygen-poor environment since they are the farthest from the portal vein. In zone three, hepatocytes are mitochondria rich and have high concentrations of glutathione (GSH) (Treinen-Moslen et al., 2001). These features contribute to the zonal patterns of liver damage commonly seen following exposure to hepatotoxicants in which one zone is preferentially damaged.

There are several resident cell types within the liver. Hepatocytes, also called the liver parenchymal cells, account for about $80-90 \%$ of liver mass and $65 \%$ of cell number of a normal liver, non-parenchymal cells including Kupffer cells (15\%), endothelial cells, hepatic stellate cells or pit cells make up the remaining mass (Blouin, Bolender \& Weibel, 1977). Hepatocytes are polygonally-shaped, polarized and highly differentiated 
cells. There is an abundance of mitochondria and they often contain a second nucleus to manage their extensive roles in energy production, protein synthesis, metabolism, and detoxification (MacSween et al., 2002).

Owing to the liver's central role in the metabolism of xenobiotic compounds, hepatotoxicity is a major issue in chemical- and drug-induced toxicology (Bhattacharya et al., 2012). Blood carrying the toxicants is filtered by the liver before being distributed to other parts of the body. The high rate of blood flow to the liver leads to delivery of high metabolic activity makes the liver a major target organ of toxicants. Therefore the liver is prone to injury in spite of its capacity for regeneration growth (Saukkonen et al., 2006). Chemical induced liver toxicity can have severe consequences. Liver pathophysiology serves as an important tool for identifying and characterizing liver injury. A broad variety of liver pathophysiologies induced by chemicals have been reported, including necrosis, hepatitis, cholestasis, steatosis, fibrosis, granuloma, vascular lesions, or neoplasm (Kaplowitz, 2004). The pathological symptoms of hepatotoxicity may allow conclusions about the affected intracellular organelles. Mechanisms of hepatotoxicity are complex and might involve different interacting pathways. Currently, there is a lack of reasonable understanding of the general biochemical mechanisms of chemical- and drug-induced hepatotoxicity (Pandit et al., 2012). Some of general mechanisms that have been proposed including cellular stress, mitochondrial dysfunction, immune reactions, inhibition of specific enzymes or transporters, and biotransformation and formation of reactive metabolites (Pandit et al., 2012).

As previously discussed, human can expose to NPs through inhalation, ingestion, skin penetration, or IV injections. Whatever the route that NPs enter the body, they can 
enter the blood circulation and then be translocated to the liver. Therefore, NPs are potential hepatotoxicants (Sadaukas et al., 2007; Wang et al., 2007). To date, there are a limited number of studies on hepatotoxicity of NPs. There are a few in vitro and in vivo studies demonstrating that NPs, including silver, nano-C60, copper, titanium dioxide and iron oxide magnetic NPs, are hepatotoxic to liver cell lines and towards rodents, but little is known of the mechanisms of hepatotoxicity caused by NPs used in these studies (Hussain et al., 2005; Sayes et al., 2005; Chen et al., 2006; Wang et al., 2007; Jain et al., 2008).

In vivo studies on QD-induced hepatotoxicity have also been previously reported (Zhang et al., 2007; Liu et al., 2011). Unfortunately, assessment of QD acute toxicity to the liver in these studies was limited to only a few biological endpoints (such as cytotoxicity, glutathione peroxidase activity, and tissue histopathology) and therefore the investigators could only speculate on the mechanisms of QD-induced hepatotoxicity, without revealing the underlying mechanisms though more detailed empirical evidence.

\subsection{Mitochondria}

The mitochondrion is one of the most important organelles in the cell as it plays a critical role in cellular energy production. Other crucial roles of the mitochondrion include maintenance calcium homeostasis, involvement in intermediate metabolism, heme and steroid synthesis, generation of ROS, regulation of the cellular redox state and in the progression or initiation of cell death (McBride et al., 2006).

Mitochondria are composed of two membranes that separate two compartments, the intermembrane compartment and the inner compartment filled with mitochondrial 
matrix (Passarella, et al., 2003). The outer membrane forms a boundary separating the mitochondrion from the cytosol. This membrane consists of numerous integral proteins and phospholipids as well as enzymes taking part to the metabolism of several substances and to the transportation of the fatty acids into the mitochondrial matrix. The inner membrane encloses and convolutes into the mitochondrial matrix, forming cristae. This membrane is less permeable to ions and small molecules than the outer membrane, thus acts as an electrical insulator and chemical barrier (Passarella, et al., 2003).

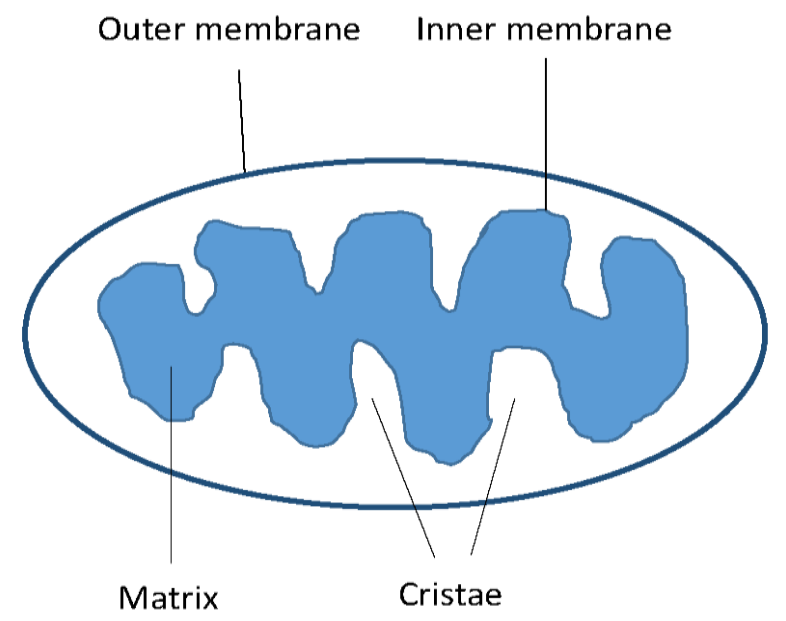

Figure 1.2. Basic structure of a mitochondrion

Mitochondrial energy production is achieved by electron transfer in the respiratory chain by using a process called oxidative phosphorylation (OXPHOS). In the matrix, tricarboxylic acid cycle (TCA) enzymes generate electron carriers (NADH and $\mathrm{FADH}_{2}$ ), which donate electrons to the electron transport chain (ETC). The ETC is located in the inner mitochondrial membrane and consists of five protein complexes: Complex I (NADH-ubiquinone oxidoreductase), Complex II (succinate-ubiquinone oxidoreductase), Complex III (ubiquinone-cytochrome $c$ reductase or cytochrome $b c_{1}$ 
complex), Complex IV (cytochrome $c$ oxidase), and Complex V (ATP synthase) (Galante et al., 1979). In oxidative phosphorylation, Complex I to IV undergo sequential redox reactions and pump protons from the matrix into the intermembrane space. Therefore, electrons are transferred along Complex I to IV from low redox potential to high redox potential, creating a proton gradient across the inner mitochondrial membrane, which is used by Complex V to drive phosphorylation of ADP to ATP (Duchen, 2004). Beyond ATP production, the inner-membrane electrochemical potential generated by OXPHOS is a vital feature of the organelle (Mitchell, 1961). The inner transmembrane potential $(\Delta \Psi \mathrm{m})$ is negative $(-180 \mathrm{mV})$ and is being maintained by pumping protons into the intermembraneous space. Membrane potential is harnessed for other essential mitochondrial functions, such as mitochondrial protein import (Neupert and Herrmann, 2007), and is used to trigger changes on the molecular level that alter mitochondrial behaviors in response to mitochondrial dysfunction. The exquisite structural and functional characteristics of mitochondria provide a number of primary targets for xenobiotic-induced bioenergetic failure, and mitochondrial damage could lead to changes in cell function and trigger cell death. (Wojtczak and Zabłocki, 2008).

Recent studies have suggested that the mitochondria are potential target organelles in NP toxicity (Nel et al., 2006 and Xia et al., 2006). NPs such as silvercoated gold, fullerenes, and multi-walled nanotubes have been reported to localize near or within mitochondria (Oberdoster et al., 2005, Foley et al., 2002, and Zhu et al., 2006). Gold NPs and diesel exhaust particles have been shown to cause mitochondrial swelling and permeability transition pore opening (Salnikov et al., 2007 and Xia et al., 2006). However, there is little known information on exactly how NPs interact with the 
mitochondria and affect theirs function. Mictochondria have also been suggested as the intracellular targets of QDs ( $\mathrm{Li}$ et al., 2011); however, there have not been any reports on the detail interaction and association of these NPs with the organelles, as well as the detection of QDs or their components inside mitochondria.

\subsection{Rationales for the research}

Human exposure to NPs is becoming unavoidable and increasing due to the rapid expansion of nanotechnology. Thus, there is an urgent need to evaluate the health risks associated with these materials. Studying the mechanisms of toxicity of NPs will provide a basis for classifying materials for regulatory purposes and prescribing strategies for risk management (Thomas et al., 2006). Cadmium-based QDs are widely used in microelectronics and biomedical research and offer great potential in medical imaging, diagnosis, and therapeutic targeting (De Wild et al., 2003; Gao et al., 2004; Jamieson et al., 2007). Even though previous studies have reported the toxicity of these NPs, the mechanisms related to their toxic effects in cell lines and in animals are still unclear $(\mathrm{Wu}$ et al., 2003; Derfus et al., 2004; Kirchner et al., 2005; Samia et al., 2006; Lovric et al., 2005; Hardman, 2006). Studying the hepatotoxicity of CdTe-QDs and their effects on mitochondria will help us to understand the physiological and intracellular behavior of these particles within target tissues and cells as well as the mechanism of toxicity that they exert on cells, tissues and the human body. The research will help to clarify the risks of the existing products and more advanced designs of CdTe-QDs on human health and environment. 


\subsection{Thesis hypothesis and objectives}

\subsubsection{Hypothesis}

Owing to its central role in the metabolism of xenobiotic compounds, the liver tends to be the major target organ of toxicants and is prone to injury. One of the mechanisms of hepatotoxicity is mitochondrial dysfunction. Since CdTe-QDs have been shown to produce ROS and free $\mathrm{Cd}^{2+}$ ions, both of which have been reported to induce hepatotoxicity via mitochondrial pathways (Samuni et al., 2010; Bertin and Averbeck, 2006), the hypothesis of this research is that CdTe-QD is a hepatotoxin and that mitochondrial toxicity is the underlying mechanism by which CdTe-QDs cause hepatotoxicity in test models.

\subsubsection{Aim and objectives}

The general aim of this research is to investigate the mechanisms of CdTe-QD induced hepatotoxicity in vitro and in vivo. The specific objectives of this research are:

1. To investigate the toxic effects of CdTe-QDs in a hepatocyte cell line.

2. To demonstrate mitochondrial toxicity as a mechanism leading to cell death in the test cell line.

3. To study systemic effects and hepatotoxicity of CdTe-QDs in a mouse model.

4. To determine the effects on mitochondria of hepatocytes in the liver as a mechanism resulting in liver injury. 


\subsubsection{Research work layout}

To achieve the set objectives, the work of this research was divided into four chapters.

Chapter 2: this chapter studied the toxicity of CdTe-QDs in hepatocellular carcinoma HepG2 cells. Upon exposure of HepG2 cells to CdTe-QDs, the following endpoints were examined: cytotoxicity, ROS generation, oxidative stress, and apoptosis. In addition, the biological factors that contribute to CdTe-QD toxicity were also investigated in this chapter.

Chapter 3: this chapter studied the effects of CdTe-QDs on mitochondria to elucidate the underlying mechanisms of CdTe-QD toxicity in HepG2 cells. Following exposure of HepG2 cells to CdTe-QDs, the effects of CdTe-QDs on mitochondrial integrity and function, including mitochondrial morphology, membrane potential, calcium homeostasis, electron transport chain, ATP production, cellular respiration, and mitochondrial biogenesis, were examined.

Chapter 4: this chapter studied systemic effects and hepatotoxicity of CdTe-QDs in $\mathrm{BALB} / \mathrm{c}$ mice. Mice were exposed to CdTe-QDs at different concentrations via intravenous. The following endpoints were examined: animal physical appearance and behaviour, distribution of CdTe-QDs in tissues, inflammation, acute phase response, tissue histology, and general liver function.

Chapter 5: this chapter studied underlying mechanisms of hepatotoxicity induced by CdTe-QDs in BALB/c mice. For this purpose, mechanism-driven endpoints such as liver oxidative stress and apoptosis along with changes in mitochondrial morphology, 
ETC, ATP production, and mitochondrial biogenesis in hepatocytes in the liver, were examined. 


\section{Chapter: Cadmium Telluride Quantum Dots Cause Oxidative Stress Leading to Extrinsic and Intrinsic Apoptosis in Hepatocellular Carcinoma HepG2 Cells}

This Chapter is adapted from the following article:

Kathy C Nguyen, William G Willmore, and Azam F Tayabali. 2013. Cadmium Telluride Quantum Dots Cause Oxidative Stress Leading to Extrinsic and Intrinsic Apoptosis in Hepatocellular Carcinoma HepG2 Cells. Toxicology. 306: 114-123.

Permission was obtained from Toxicology journal and Government of Canada for incorporation of this paper into this thesis. Minor changes were performed to the formatting of the document so that it could fit in the format of this dissertation.

For this paper, Kathy C. Nguyen designed and conducted these experiments, and wrote the manuscript. Drs. Azam Tayabali and William Willmore provided input into the manuscript. Dr. Azam Tayabali and Health Canada provided funding for the project. 


\subsection{Abstract}

The mechanisms of toxicity related to human hepatocellular carcinoma HepG2 cell exposures to cadmium telluride quantum dots (CdTe-QDs) were investigated. CdTeQDs caused cytotoxicity in HepG2 cells in a dose- and time-dependent manner. Treated cells showed an increase in reactive oxygen species (ROS). Altered antioxidant levels were demonstrated by depletion of reduced glutathione (GSH), a decreased ratio of reduced glutathione to oxidized glutathione (GSH/GSSG) and an increased NF-E2related Factor 2 (Nrf2) activation. Enzyme assays showed that superoxide dismutase (SOD) activity was elevated whereas catalase (CAT) and glutathione-S-transferase (GST) activities were depressed. Further analyses revealed that CdTe-QD exposure resulted in apoptosis, indicated by changes in levels of caspase-3 activity, poly ADP-ribose polymerase (PARP) cleavage and phosphatidylserine externalization. Extrinsic apoptotic pathway markers such as Fas levels and caspase- 8 activity increased as a result of CdTeQD exposure. Involvement of the intrinsic/mitochondrial apoptotic pathway was indicated by decreased levels of B-cell lymphoma $2(\mathrm{Bcl} 2)$ protein, and mitochondrial cytochrome c and increased levels of mitochondrial Bcl-2-associated X protein (Bax) and cytosolic cytochrome c. Further, mitogen-activated protein kinases (MAPKs) such as c-Jun N-terminal Kinases (JNK), extracellular signal-regulated kinases (Erk1/2), and p38 were all activated. Our findings reveal that CdTe-QDs cause oxidative stress, interfere with antioxidant defenses and activate protein kinases, leading to apoptosis via both extrinsic and intrinsic pathways. Since the effects of CdTe-QDs on selected biomarkers were similar or greater compared to those of $\mathrm{CdCl}_{2}$ at equivalent 
concentrations of cadmium, the study suggests that the toxicity of CdTe-QDs arises from a combination of the effects of cadmium and ROS generated from the NPs.

\subsection{Introduction}

Quantum dots (QDs) are engineered semiconductor nanocrystals that range from 2-100 $\mathrm{nm}$ in diameter (Bruchez et al., 1998). The usefulness and various applications of QDs have led to elevated levels of production of these NPs, resulting in potentially significant increases in human exposure from occupational, medical and environmental sources. Although, there is a paucity of information on actual environmental and occupational levels of exposure to CdTe-QDs, there are increasing concerns about their toxicity and risk to human health. Cadmium-based QDs such as CdSe and CdTe have been shown to cause toxicity in vitro and in vivo (Hardman, 2006). However, the underlying mechanisms of QD-mediated toxicity are not well understood. Several studies have suggested that the toxicity of QDs depends on several intrinsic properties such as size, chemical composition, and surface coating components (Hardman 2006). Many studies have also suggested that the presence of cadmium in the QD metal core is a primary source of QD toxicity (Derfus et al., 2004; Chang et al., 2006). The release of $\mathrm{Cd}^{2+}$ ions from QDs is hypothesized to result from the degradation or oxidation of the metal core, and the amount of free $\mathrm{Cd}^{2+}$ ions in solutions of QDs correlates with QDinduced cytotoxicity (Derfus et al., 2004; Kirchner et al., 2005; Xu et al., 2010). Another source of toxicity of QDs is the generation of reactive oxygen species (ROS) (Samia et al., 2006). QDs are thought to have the ability to spontaneously induce ROS production because of their electron configuration (Maysinger and Lovric, 2007). ROS generation in 
cells can result in oxidative stress and affect cellular signaling cascades that control different cellular processes leading to cell damage and triggering apoptosis (Simon et al., 2000).

Apoptosis can occur by means of extrinsic and intrinsic (mitochondria-dependent) pathways (Putcha et al., 2002). The former signals involve binding of TNF- $\alpha$ or Fas ligand to their receptors leading to activation of the protease caspase-8, which either directly cleaves and activates the effector caspases, or indirectly activates the downstream caspases through the cleavage of BH3-only protein Bid (Luo et al., 1998). The induction of the intrinsic pathway involves decreased anti-apoptotic signals such as Bcl2 and translocation of pro-apoptotic signals such as Bax and Bak to mitochondria. These events lead to release of cytochrome $\mathrm{c}$ and other apoptosis-inducing factors from the mitochondria into the cytosol to trigger subsequent activation of procaspase- 9 and downstream apoptotic effectors (Crompton, 2000). Although there are a growing number of reports on the toxicity of CdTe-QDs including causing oxidative stress and apoptosis, it is currently still not clear whether $\mathrm{Cd}^{2+}$ ions, ROS, or both are the key factors in the toxicity induced by CdTe-QDs, and what pathways are involved in the mechanisms leading to cell death.

The aim of this study is to investigate the mechanisms of CdTe-QD induced toxicity in hepatocellular carcinoma HepG2 cells. This cell line is considered a suitable model to study in vitro xenobiotic metabolism and potential hepatotoxicity, since it retains many specialized functions indicative of normal human hepatocytes (Knowles et al., 1980). HepG2 cells have been used as a tool for studying genotoxicity, oxidative stress, mitochondrial dysfunction and apoptosis (Knasmuller et al., 2004). In this study, 
some assays involved measurements made directly in situ (imaging test cells by confocal microscopy) and others were made by in vitro methods to assess changes in several key biomarkers which are indicative of oxidative stress, onset of apoptosis (markers for both extrinsic and intrinsic apoptotic pathways), and phosphorylation of signal transduction kinases. In addition, the effects of CdTe-QDs on key HepG2 response biomarkers were compared to those obtained in similar exposures using equivalent amounts of cadmium in form of $\mathrm{CdCl}_{2}$. Overall, the study reveals that $\mathrm{CdTe}-\mathrm{QDs}$ cause oxidative stress, interfere with antioxidant defenses and activate protein kinases, leading to apoptosis via both extrinsic and intrinsic pathways. The results suggest that the toxicity of these NPs might be induced from both cadmium effects and ROS generation.

\subsection{Materials and Methods}

\subsubsection{Materials}

HepG2 cells were obtained from American Type Culture Collection (ATCC) (Manassas, VA). CdTe-QDs were purchased from Nano Impex Canada (Mississauga, ON). CdTe-QDs were described by the manufacturer as CdTe/CdS core/shell QDs, encapsulated by polyacrylate polymer layers, with a size of $5 \mathrm{~nm}$, a spectral emission of $540 \mathrm{~nm}$ and a concentration of $10 \mathrm{mg} / \mathrm{ml}$ in water containing $10 \%$ of cadmium. MTT [3(4,5-dimethylthiazol-2-yl) 2,5-diphenyl tetrazolium bromide], dimethyl sulfoxide (DMSO), cadmium chloride $\left(\mathrm{CdCl}_{2}\right)$, dihydroethidium (DHE), menadione and staurosporine (STS) were obtained from Sigma-Aldrich (St. Louis, MO). Eagle's Minimum Essential Medium (EMEM), fetal bovine serum and gentamicin were obtained from Invitrogen (Carlsbad, CA). 


\subsubsection{Spectral and size characterization of CdTe-QDs}

Characterization of test CdTe-QDs were carried out as described in a previous study (Nguyen et al., 2013). For spectral characterization, fluorescence spectra of CdTeQDs (450 nm excitation and emission wavelengths from $450 \mathrm{~nm}$ to $650 \mathrm{~nm}$ ) were obtained by using $100 \mu \mathrm{l}(100 \mu \mathrm{g} / \mathrm{ml})$ of the NP solution in a 96-well opaque plate and scanning with a SPECTRAmax GEMINI XS microplate spectrofluorometer (Molecular Devices, Sunnyvale, CA). A standard curve of fluorescence intensity (measured at 540 nm) vs. concentration was made test CdTe-QDs using three replicates of serial dilutions ranging from $1.562 \mu \mathrm{g} / \mathrm{ml}$ to $50 \mu \mathrm{g} / \mathrm{ml}$.

Size characterization of CdTe-QDs was performed using atomic force microscopy (AFM) and transmission electron microscopy (TEM). For observation by AFM, commercial stocks and serial dilutions of CdTe-QDs made in $\mathrm{ddH}_{2} \mathrm{O}$ were deposited on freshly cleaved mica. Samples were air-dried overnight and were imaged in non-contact

mode with a PPP-NCH probe (Nanosensors ${ }^{\mathrm{TM}}$, Switzerland) with an Agilent 5500 AFM. For TEM, CdTe-QD stocks were diluted by 1,000-fold with $\mathrm{ddH}_{2} \mathrm{O}, \mathrm{PBS}$, or culture medium (DMEM), deposited on formvar-coated grids, and air-dried overnight at RT. Grids were examined with a JOEL JEM 1230 operating at $60 \mathrm{kV}$. AFM images were quantified using Picoview software (version 1.2.4) provided with the microscope. For TEM analysis, micrographs were imported into an image analysis package for size determination (Nikon NIS Elements Basic Research).

\subsubsection{Cell culture conditions and treatments}


HepG2 cells were cultured in EMEM supplemented with $10 \%$ fetal bovine serum (FBS) and $100 \mu \mathrm{g} / \mathrm{ml}$ gentamicin at $37^{\circ} \mathrm{C}$ in a humidified atmosphere with $5 \% \mathrm{CO}_{2}$. For the MTT assay, cells were seeded into 96 -well plates at density of $5 \times 10^{4}$ cells $/ 100 \mu \mathrm{L}$ well. For confocal microscopy, cells were seeded on cover-slips placed in a 12-well plate, at a concentration of $1 \times 10^{5}$ cells $/$ well in $1 \mathrm{ml}$ of medium. For enzyme-linked immunosorbent assay (ELISA), bead array and enzymatic assays, cells were seeded into 6-well or 96 -well plates at density of $5 \times 10^{5}$ cells $/ 2 \mathrm{ml}$ well or $5 \times 10^{4}$ cells $/ 100 \mu 1$ well, respectively. Cells were cultured for $24 \mathrm{~h}$ to $80 \%$ confluency and the medium was replaced just prior to CdTe-QDs exposure.

Working solutions of CdTe-QDs and $\mathrm{CdCl}_{2}$ were prepared by diluting the stock solution in phosphate-buffered saline (PBS). For the MTT assay, cells were treated with different concentrations of CdTe-QD $(0.001 \mu \mathrm{g} / \mathrm{ml}$ to $10 \mu \mathrm{g} / \mathrm{ml})$ for different durations. For other assays, cells were treated with $10 \mu \mathrm{g} / \mathrm{ml} \mathrm{CdTe-QDs} \mathrm{(containing} 1 \mu \mathrm{g} / \mathrm{ml}$ of cadmium) for $24 \mathrm{~h}$. PBS alone was used for sham treatments. Except for MTT assay, treatments with $1.63 \mu \mathrm{g} / \mathrm{ml}$ of $\mathrm{CdCl}_{2}$ (containing $1 \mu \mathrm{g} / \mathrm{ml}$ of cadmium) was done in all other assays for comparison purposes. Menadione $(25 \mu \mathrm{M})$ was used as a positive control in ROS detection assays, and STS $(1 \mu \mathrm{M})$ was used as the positive control for caspase-3, cleaved PARP, annexin V, Fas, caspase-8, Bax, Bcl2, cytochrome c and phosphoprotein assays. For each enzymatic and ELISA assay, the same protein concentrations of the control and treated samples were used. All measurements were conducted in duplicate in three independent experiments. 


\subsubsection{MTT assay}

The MTT assay was conducted as described in Nguyen et al. (2012). Briefly, following the treatment of cells with CdTe-QDs, medium was removed and replaced with fresh medium $(100 \mu \mathrm{l} /$ well $)$. A total of $10 \mu \mathrm{l}$ stock MTT $(10 \mathrm{mg} / \mathrm{ml})$ was added to each well and cells were incubated for $1 \mathrm{~h}$ at $37^{\circ} \mathrm{C}$. Media was removed and cells were rinsed with PBS (100 $\mu \mathrm{l} /$ well). Cells were lysed and formazan was solubilised with DMSO (100 $\mu \mathrm{l} /$ well). Absorbance was measured at $505 \mathrm{~nm}$ using a multiwell scanning spectrophotometer (Molecular Devices, Sunnyvale, CA).

\subsubsection{In situ ROS detection}

Cells were grown to $80 \%$ confluency on glass cover-slips inside 12 -well tissue culture plates. After treatment, cells were rinsed with PBS and incubated with dihydroethidium (DHE) at concentration of $30 \mu \mathrm{M}$ for $30 \mathrm{~min}$. Cells were again washed with PBS and the cover-slip containing the monolayer of cells was mounted on a slide and viewed immediately with a Nikon TE2000 microscope attached to a C1 confocal unit (Nikon Canada Inc. Mississauga ON). Fluorescence areas from confocal micrographs

were analysed using Nikon Imaging Software (NIS) element (Nikon Canada Inc. Mississauga $\mathrm{ON}$ ). The average area of fluorescence of three micrographs was plotted to quantify the level of ROS production in the control and treatments.

\subsubsection{Reduced GSH and GSH/GSSG ratio assay}

The cellular-reduced GSH concentration was assayed using a glutathione colorimetric assay kit (Oxford Biomedical Research, Oxford, MI) according to the 
manufacturer's protocol. The GSSG concentration was measured according to the method by Griffith (1980) with modification. Briefly, $2 \mu$ l of 2-vinylpyridine (0.5 M) was added to $100 \mu \mathrm{l}$ of each cell lysate sample and the plate was frozen at $-80^{\circ} \mathrm{C}$ and subsequently thawed at $37^{\circ} \mathrm{C}$. Each sample $(50 \mu 1)$ was transferred into a new microtiter well followed by $60 \mu$ of reduced nicotinamide adenine dinucleotide phosphate (NADPH) (0.35 mM), $10 \mu \mathrm{l}$ of 5, 5'-dithio-bis(2-nitrobenzoic acid) (DTNB), (6 mM), and $10 \mu \mathrm{l}$ of glutathione reductase $(5 \mathrm{IU} / \mathrm{ml})$. The microtiter plate was incubated for 1 min at RT and the absorbance was measured at $405 \mathrm{~nm}$. The levels of reduced GSH and GSSG were calculated by using a standard curve obtained with reduced GSH and GSSG. The content of reduced GSH was obtained by subtracting the amount of 2xGSSG from the total GSH level.

\subsubsection{SOD assay}

After CdTe-QD treatment, cells were collected, homogenized in cold $20 \mathrm{mM} 4-2-$ hydroxyethyl)-1-piperazineethanesulfonic (HEPES) buffer ( $\mathrm{pH}$ 7.2) containing $1 \mathrm{mM}$ ethylene glycol tetraacetic acid (EGTA), $210 \mathrm{mM}$ mannitol, and $70 \mathrm{mM}$ sucrose, and

centrifuged at $1,500 \mathrm{x}$ for $5 \mathrm{~min}$ at $4^{\circ} \mathrm{C}$. The supernatants were collected and centrifuged at $10,000 \times \mathrm{g}$ for $15 \mathrm{~min}$ at $4^{\circ} \mathrm{C}$ to yield cytosolic SOD samples. The pellets were homogenized in cold HEPES buffer to yield mitochondrial SOD samples. SOD samples were assayed using a SOD colorimetric enzyme assay kit from Cayman Chemical (Ann Arbor, MI) according to the manufacturer's protocol.

\subsubsection{Nrf2 activation assay}


The activation of Nrf2 transcription factor was measured using an ELISA kit from Active Motif, (Carlsbad, CA). After treatments, cells were washed and subjected to a nuclear extraction procedure according to the manufacturer's instruction. Briefly, in a provided microplate, $10 \mu \mathrm{L}$ of each nuclear extract was added to the assigned well containing $40 \mu 1$ of binding buffer. The plate was incubated for $1 \mathrm{~h}$ at RT. After three washes, primary antibody $(100 \mu \mathrm{l})$ was added to each well followed by a $1 \mathrm{~h}$ incubation and three washes. The procedure was repeated with the horseradish peroxidase (HRP)conjugated secondary antibody. Colorimetric reaction was initiated by adding $100 \mu 1$ of developing solution to each well. The color was monitored visually and the reaction was stopped by adding $100 \mu 1$ of stop solution. The color change was measured at $450 \mathrm{~nm}$ using a spectrophotometer (Molecular Devices, Sunnyvale, CA).

\subsubsection{Catalase (CAT) assay}

CAT activity was measured using a catalase assay kit from Cayman Chemical (Ann Arbor, MI). In brief, cells were collected after treatment and sonicated in a cold buffer containing $50 \mathrm{mM}$ potassium phosphate $(\mathrm{pH}$ 7.0) and 1 mM ethylenediaminetetraacetic acid (EDTA). The supernatants were collected after centrifugation at $10,000 \mathrm{x}$ g for $15 \mathrm{~min}$ at $4^{\circ} \mathrm{C}$. To each well containing $20 \mu 1$ of standard, control, or sample, $100 \mu \mathrm{l}$ of assay buffer $(100 \mathrm{mM}$ potassium phosphate, $\mathrm{pH} 7.0$, containing $1 \mathrm{mM}$ EDTA) and $30 \mu 1$ of methanol were added. $\mathrm{H}_{2} \mathrm{O}_{2}(20 \mu \mathrm{l})$ was added to each well. After the plate was incubated for $20 \mathrm{~min}$ at RT, $30 \mu 1$ of potassium hydroxide followed by $30 \mu \mathrm{l}$ of purpald was added to each well. The plate was then incubated for 
$10 \mathrm{~min}$ at RT. Finally, $10 \mu \mathrm{l}$ of potassium periodate was added to each well and the plate was incubated at RT for 5 min before measurement with a spectrophotometer at $540 \mathrm{~nm}$.

\subsubsection{GST activity and quantification assays}

GST activity was measured using GST enzymatic kit from Arbor Assays (Ann Arbor, MI) according to the manufacturer's instructions. Briefly, cells were lysed in the assay buffer and centrifuged at $1,500 \mathrm{xg}$ for $5 \mathrm{~min}$, at $4^{\circ} \mathrm{C}$ to obtain supernatant samples. Each sample or standard $(50 \mu \mathrm{l})$ was added to the assigned well followed by $25 \mu \mathrm{l}$ of detection buffer and $25 \mu \mathrm{l}$ of GSH. The plate was incubated for $30 \mathrm{~min}$ at RT and then measured on a spectrofluorimeter with excitation at $390 \mathrm{~nm}$ and emission at $450 \mathrm{~nm}$.

For quantification of GST- $\alpha$, cells were lysed in PBS supplemented with 1 x protease inhibitors and centrifuged at $1,500 \mathrm{x}$ g for $5 \mathrm{~min}$ at $4^{\circ} \mathrm{C}$ to collect the supernatants which were assessed for protein concentration and used in the GST- $\alpha$ ELISA assay using a kit from Oxford Biomedical Research (Oxford, MI) following the manufacturer's instructions.

\subsubsection{Caspase-3 activity and cleaved PARP assay}

Activated caspase-3 and cleaved PARP levels were measured using bead plex assay kits from EMD Millipore Corporation (Billerica, MA) according to the manufacturer's instruction. After treatments, cells were homogenized in provided lysis buffer and centrifuged at $12,000 \mathrm{rpm}$ for $20 \mathrm{~min}$ at $4^{\circ} \mathrm{C}$. The supernatants were collected, measured for protein concentration and diluted in assay buffer. Sample $(25 \mu 1)$ was added to the corresponding well in a 96-well filter plate containing $25 \mu 1$ of anti-caspase- 
3 or PARP conjugated beads. The plate was incubated at $4^{\circ} \mathrm{C}$ overnight. After washing, $25 \mu 1$ of detection antibody was added to each well and the plate was incubated for $1 \mathrm{~h}$ at RT. Detection antibody was removed by vacuum filtration and $25 \mu 1$ of pre-diluted streptavidin-conjugated phycoerythrin was added to each well. The plate was incubated for $15 \mathrm{~min}$ at RT on a shaker. After vacuum filtration, $120 \mu 1$ of assay buffer was added to each well. The plate was shaken for 1 min and analyzed with the Bio-Plex 100 Array System (Bio-Rad, Hercules, CA).

\subsubsection{Annexin V staining assay}

Cells were seeded onto coverslips in a 12 -well plate overnight and grown to $80 \%$ confluency. After cells were treated with CdTe-QDs and positive controls, cells were washed with PBS and incubated with annexin V solution containing $1 \mu \mathrm{g} / \mathrm{ml}$ of annexin V-phycoerythrin in annexin V binding buffer (R\&D Systems, Minneapolis, MN) for 10 min at $37^{\circ} \mathrm{C}$. Cells were washed twice with PBS and fixed with $4 \%$ paraformaldehyde containing $0.1 \%$ Triton X-100 for 10 min. After that, cells were washed twice with PBS and incubated with Sytox $\operatorname{Red}^{\mathrm{TM}}(1: 1,000)$ for $15 \mathrm{~min}$. After washing, cover slips were mounted on slides and dried overnight before being observed with a Nikon TE2000 microscope attached to a $\mathrm{C} 1$ confocal unit.

\subsubsection{Fas detection assay}

Fas level was measured using a Fas ELISA assay kit from Promokine (PromoCell GmbH, Heidelberg, Germany). Briefly, after treatments, cells were homogenized in lysis buffer (20 mM Tris, $\mathrm{pH} 7.4,137 \mathrm{mM} \mathrm{NaCl}, 1 \% \mathrm{NP}-40)$ containing protease inhibitors. 
Cell lysates were vortexed and centrifuged at $12,000 \mathrm{rpm}$ for $15 \mathrm{~min}$ at $4^{\circ} \mathrm{C}$. The supernatants were collected, measured for protein concentration and used in the ELISA assay, following the manufacturer's instructions.

\subsubsection{Caspase-8 activity}

Caspase- 8 activity was measured using a caspase- 8 enzymatic assay kit from Abcam (Cambridge, MA). Briefly, after treatments, cells were resuspended in chilled cell lysis buffer and incubated on ice for $10 \mathrm{~min}$. Cell lysates were centrifuged for $1 \mathrm{~min}$ at $10,000 \mathrm{x}$ g. Supernatants were collected, measured for protein concentration and placed on ice until analysis. For each sample, $100 \mu \mathrm{g}$ of protein was used and diluted in $50 \mu 1$ of cell lysis buffer. To each sample, $50 \mu 1$ of provided $2 \mathrm{X}$ reaction buffer and $5 \mu 1$ of the $4 \mathrm{mM}$ acetyl-Ile-Glu-Thr-Asp p-nitroaniline (IETD-pNA) substrate were added. The samples were incubated at $37^{\circ} \mathrm{C}$ for $1 \mathrm{~h}$, transferred to a microtiter plate and read at $405 \mathrm{~nm}$ in a microplate reader.

\subsubsection{Bcl2 detection assay}

Bcl2 level was measured using bead plex assay kit from EMD Millipore Corporation (Billerica, MA) according to the manufacturer's protocol. After treatments, cells were homogenized in the lysis buffer provided and centrifuged at 12,000 rpm for 20 min at $4^{\circ} \mathrm{C}$. The supernatants were collected and diluted in assay buffer. Each standard or sample $(25 \mu \mathrm{l})$ was added to the corresponding well in a 96 -well filter plate containing $25 \mu \mathrm{l}$ of anti-Bcl 2 conjugated beads. The plate was incubated at $4{ }^{\circ} \mathrm{C}$ overnight. After three washes with wash buffer, $25 \mu$ of detection antibody was added to each well and 
the plate was incubated for $1 \mathrm{~h}$ at RT. Detection antibody was removed by vacuum filtration and $25 \mu 1$ of pre-diluted streptavidin-conjugated phycoerythrin was added to each well. The plate was incubated for $15 \mathrm{~min}$ at RT on a shaker. After vacuum filtration, $120 \mu \mathrm{l}$ of assay buffer was added to each well. The plate was shaken for $1 \mathrm{~min}$ and analyzed with the Bio-Plex 100 Array System (Bio-Rad, Hercules, CA).

\subsubsection{Bax detection assay}

Bax levels were measured using a Bax Enzyme Immunometric Assay (EIA) kit from Enzo Life Sciences Incorporated (Farmingdale, NY). After treatments, cells were rinsed with ice-cold PBS and subjected to the subcellular fractionation assay using a mitochondria/cytosol fractionation kit, following the manufacturer's protocol. Subcellular fractions were measured for protein concentration and used as the samples for the EIA assay which was performed according to the kit's instructions.

\subsubsection{Cytochrome c assay}

Levels of cytosolic and mitochondrial cytochrome c were measured using a cytochrome c EIA kit from Enzo Life Sciences Incorporated (Farmingdale, NY). After treatments, cells were rinsed with ice-cold PBS and subjected to the subcellular fractionation assay using the mitochondria/cytosol fractionation kit, following the manufacturer's protocol. Samples (cytosolic and mitochondrial fractions) were measured for protein concentration and used as the samples for the EIA assay which was performed according to the kit's instructions. 


\subsubsection{Phosphoprotein detection assay}

Phosphoprotein measurement was done using multiplex bead phosphoprotein assay kit from Bio-Rad (Hercules, CA), according to the manufacturer's protocol. Briefly, after treatment, cells were lysed in provided lysis buffer containing protease inhibitors, centrifuged at $12,000 \mathrm{rpm}$ for $20 \mathrm{~min}$ at $4^{\circ} \mathrm{C}$. The supernatants were collected and measured for protein concentration and diluted in sample diluent buffer provided. Anti-phosphoprotein conjugated beads were added to individual wells of a 96-well filter plate and adhered using vacuum filtration. After washing, $50 \mu 1$ of pre-diluted standards or supernatants were added and incubated for $30 \mathrm{~min}$ at RT with gentle shaking. The filter plate was then washed, $25 \mu 1$ of pre-diluted detection antibody was added to each well, and the plate was incubated as described above. After washing, $50 \mu 1$ of pre-diluted streptavidin-conjugated phycoerythrin was added to each well and the plate was shaken for $10 \mathrm{~min}$. The plate was washed and $125 \mu \mathrm{l}$ of assay buffer was added to each well. The plate was shaken for $1 \mathrm{~min}$ and analyzed with the Bio-Plex Array System (Bio-Rad, Hercules, CA).

\subsubsection{Statistical analysis}

Results were compared by one-way analysis of variance (ANOVA) followed by Dunnett's test for comparison of treatment groups to the negative control group and Tukey's test for pairwise comparisons among treatment groups. All data was expressed as mean \pm standard deviation. A value of $\mathrm{p}<0.05$ was considered as statistically significant. 


\subsection{Results}

\subsubsection{Spectral and size characterization of CdTe-QDs}

The fluorescence spectra showed the maximum emission for CdTe-QDs was at $530 \mathrm{~nm}$ (Figure 2.1.A). The standard curve was plotted using dilutions of known concentrations of CdTe-QDs. The standard curve was relatively linear as indicated by the excellent curve fit $\left(\mathrm{R}^{2}=0.997\right)$ (Figure 2.1.B).

Size characterization of CdTe-QDs was performed using AFM and TEM (Figure 2.1.C, D, E, and F). The commercial stock of CdTe-QDs were too concentrated for enumeration (Figure 2.1.C). Some larger particles were also evident which were not observed at lower concentrations. They may represent either aggregates or contaminants. A dilution of $10^{3}$-fold was observed to yield well-dispersed NPs that were amenable to analysis. For AFM measurements, only the Z-dimension was used for determination of size to avoid probe-related artifacts. These measurements yielded a mean size of $7.3 \pm$ $1.2 \mathrm{~nm}$ ranging from $5.1 \mathrm{~nm}$ to $10.1 \mathrm{~nm}$ (Figure 2.1D). TEM images were also analysed for size, by measuring minimum and maximum dimensions of each NP. With dilution in $\mathrm{ddH}_{2} \mathrm{O}$, the average particulate size in either dimension for CdTe-QDs was $14 \pm 2.8 \mathrm{~nm}$ and ranged from 6.2 to $21.5 \mathrm{~nm}$ (Figure 2.1.E and F). By contrast, TEM images of QD sample prepared with PBS indicated the presence of additional particulate-like matter related to salt crystals. However, careful analysis of grids in the areas with fewer salt crystals, test CdTe-QD were spread out evenly, and individual NPs were observed, but the TEM image was obscured by artifacts created by PBS (data not shown). Similarly, TEM particulate imaging using DMEM as the diluent was not informative because its complex components formed an electronically impenetrable mass which blocked the 
TEM beam. These results suggest that there are limitations in the use of TEM imaging of NPs when using different diluents such as buffers and media used in cell work. Using $\mathrm{ddH}_{2} \mathrm{O}$ as the diluent, the minimum and maximum diameter of each CdTe-QD, yielded an average ration of $1.18 \pm 0.03$, indicating that the shape of test CdTe-QDs is approximately spherical (Figure 2.1.E and F). The same data indicate that there is a discrepancy in QD size estimated by the two analytical methods. Alternatively, it may be that the larger sizes observed here are forms of agglomerated NPs.

A

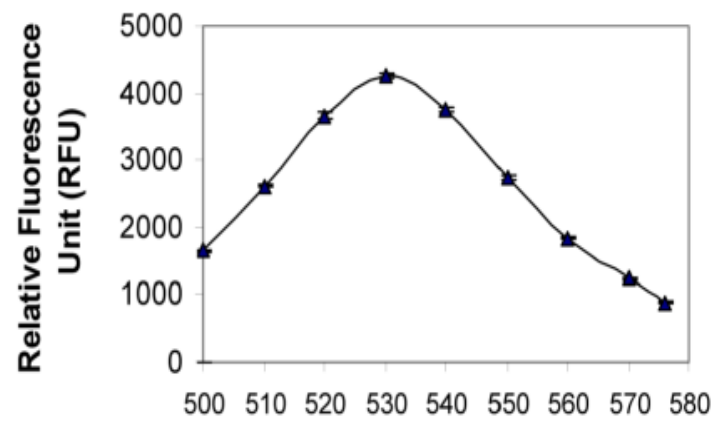

Em Wavelength in nm (Ex: 450nm)
B

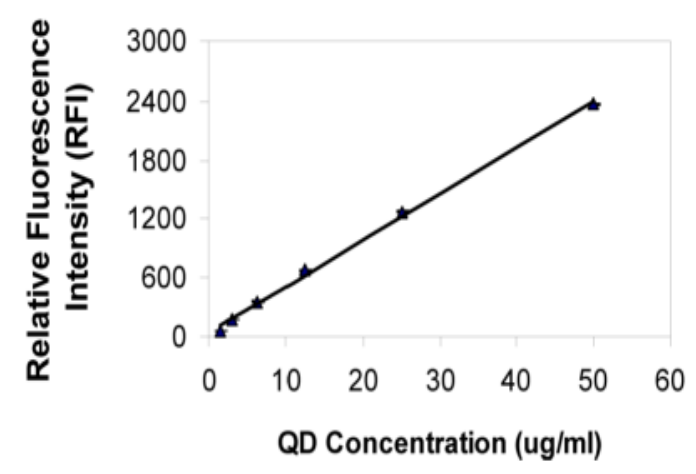


C

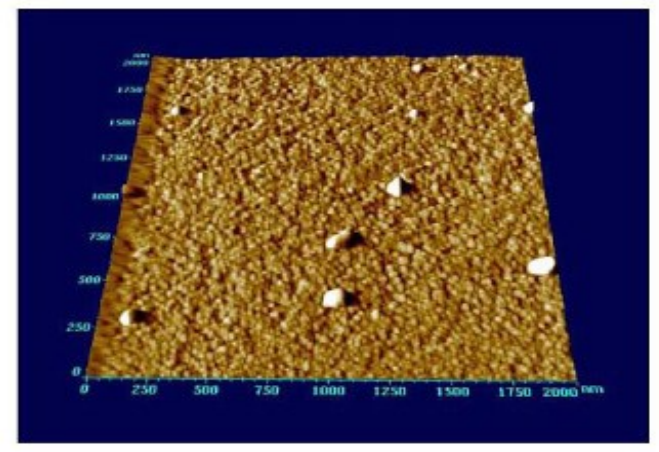

E

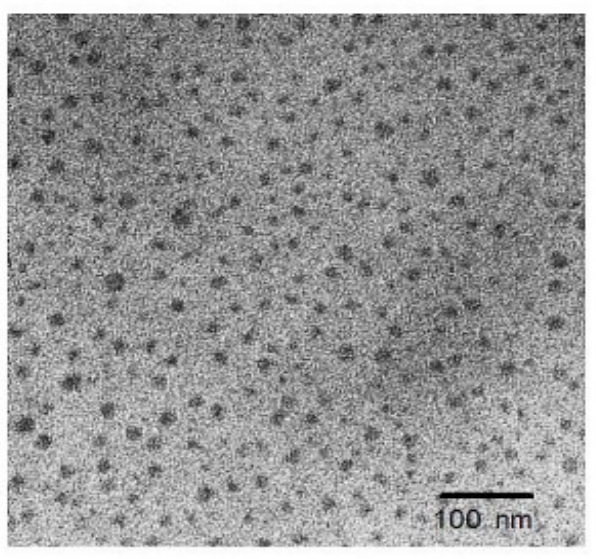

D
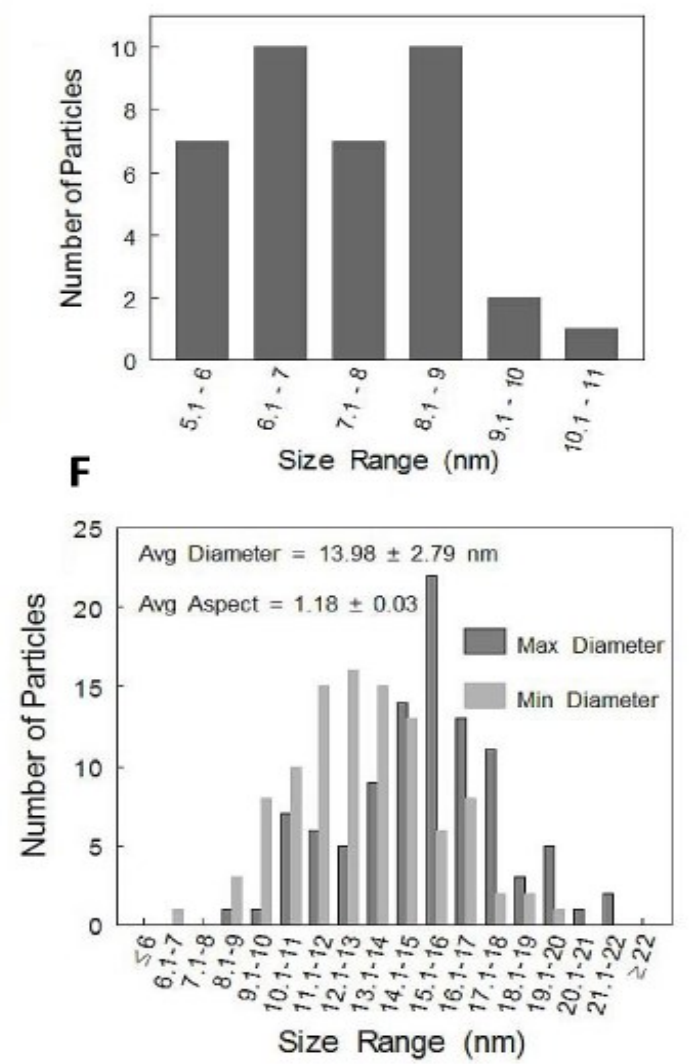

Figure 2.1. Characterization of CdTe-QDs. (A) Fluorescence spectra for CdTe-QDs at $450 \mathrm{~nm}$ excitation and emission wavelengths from $500 \mathrm{~nm}$ to $580 \mathrm{~nm}$. (B) Standard curves for CdTe-QDs at $485 \mathrm{~nm}$ excitation and $540 \mathrm{~nm}$ emission. The standard curve was plotted using linear curve fit $(\mathrm{y}=\mathrm{A}+\mathrm{Bx})$ with $\mathrm{A}=21.056, \mathrm{~B}=43.995$, and $\mathrm{R}^{2}=0.997$. (C) AFM image of full commercial concentration of CdTe-QD deposited on freshly cleaved mica. (D) Summary of Z-dimension size measurements of diluted CdTe-QDs as measured by AFM. (E) TEM image of diluted CdTe-QDs. (F) The diameter of each individual CdTe-QDs was measured at its minimum and maximum dimensions. 


\subsubsection{Cytotoxicity}

Cytotoxicity of CdTe-QDs in HepG2 cells was examined for changes in bioreducing activity using the MTT assay to estimate cellular capacity to reduce MTT to its formazan. Loss in HepG2 bioreduction induced by CdTe-QDs appeared to be timeand dose-dependent (Figure 2.2.). The earliest changes were observed at $6 \mathrm{~h}$ with 1.0 $\mu \mathrm{g} / \mathrm{ml}$, and the lowest observable effects were observed with $0.1 \mu \mathrm{g} / \mathrm{ml}$ at $12 \mathrm{~h}$ exposure. At the longest exposure duration (24 h), CdTe-QDs resulted in a significant drop in bioreduction starting at $0.1 \mu \mathrm{g} / \mathrm{ml}$, with maximal effects being $\sim 25 \%$ relative to control. Examination by microscopy showed that, even at this high dose and exposure, cells had not detached (data not shown) and most still retained at least some capacity to reduce MTT to formazan, albeit at a much lower level compared to PBS-treated controls.

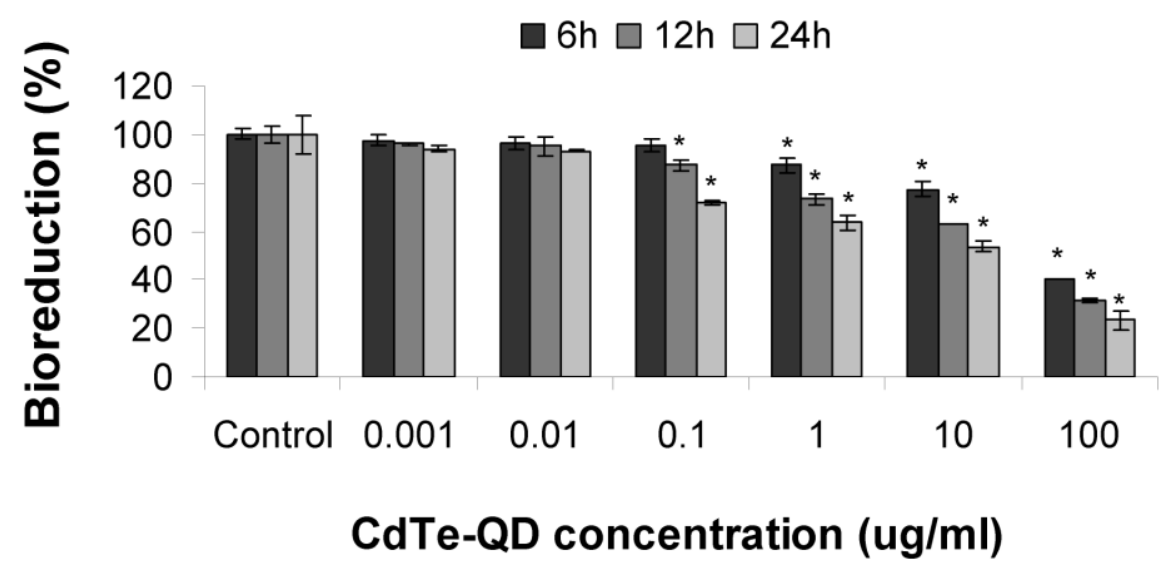

Figure 2.2. Cytotoxicity in HepG2 cells as assessed by loss of MTT bioreduction activity of CdTe-QD treated cultures relative to PBS treated controls. Data points represent the means of three independent experiments done in duplicate \pm standard deviations. The asterisks $(*)$ indicate statistically significant differences compared to PBS-treated control $(\mathrm{p}<0.001)$. 


\subsubsection{Production of ROS}

The effect of CdTe-QDs on the production of ROS was examined by observing fluorescence of oxidised DHE in HepG2 by confocal microscopy. CdTe-QD treatment resulted in increased intensity and area of fluorescence from DHE oxidation compared to PBS-controls, indicating that excess ROS levels were induced by CdTe-QDs (Figures 2.3.A, $\mathrm{B}$ and $\mathrm{E}$ ). Both $\mathrm{CdCl}_{2}$ and menadione treatments also showed an increase in ROS levels in test cells. $\mathrm{CdCl}_{2}$ treatment, however, caused a lower level of ROS generation than CdTe-QD treatment $(\mathrm{p}<0.05)$ (Figures 2.3.A, B, C, D and E).

\subsubsection{Induction of oxidative stress}

Several oxidative stress markers were selected to measure the effects of CdTeQDs on the oxidative status of HepG2 cells. Exposures of HepG2 cells to CdTe-QDs resulted in a significant depletion of reduced glutathione (GSH) (Figure 2.4.A). Furthermore, CdTe-QDs caused drops in the GSH/GSSG ratio by 2.4 -fold, compared to PBS treated controls (Figures 2.4.A and $\mathrm{B}$ ). $\mathrm{CdCl}_{2}$ induced a greater depletion of reduced GSH $(p<0.05)$, but a lower effect on the GSH/GSSG ratio compared to CdTeQDs $(\mathrm{p}<0.05)$ (Figures 2.4.A and B).

SOD activity was measured in both cytosolic and mitochondrial fractions (Figure 2.4.C). About 30\% increase in SOD activity, in both cytosolic and mitochondrial extracts, occurred with CdTe-QD treatment. $\mathrm{CdCl}_{2}$ treatment also resulted in increased cytosolic and mitochondrial SOD activities, but to a lesser extent, compared to CdTe-QD treatment. 
Nrf2 activation was found to be 2 -fold $(\mathrm{p}<0.001)$ greater in CdTe-QD-treated cells, compared with control cells (Figure 2.4.D), whereas $\mathrm{CdCl}_{2}$ caused a marginal increase (1.11-fold) in Nrf2 activation (Figure 2.4.D).

Compared to PBS-treated control cells, CdTe-QDs induced a reduction in GST activity by 1.95 -fold $(\mathrm{p}<0.001)$ and $\mathrm{CdCl}_{2}$ also caused a significant decrease in GST activity $(1.65$-fold, $\mathrm{p}<0.001)$. To determine whether the decrease in GST activity was due to CdTe-QDs reducing GST protein levels directly, quantification of GST- $\alpha$ was performed. The results showed that GST- $\alpha$ levels in the CdTe-QD treated cultures appeared to be similar to the GST- $\alpha$ levels in control cultures. Similarly, $\mathrm{CdCl}_{2}$ did not cause a change in GST- $\alpha$ levels (Figure 2.5.A).

CAT activity measurements showed that treatment with CdTe-QDs resulted in a significant decrease $(1.4$-fold, $\mathrm{p}<0.001)$ in this enzyme activity, compared to the control (Figure 2.5.B). Treatment of $\mathrm{CdCl}_{2}$ also resulted in a similar reduction in CAT activity (Figure 2.5.B). 

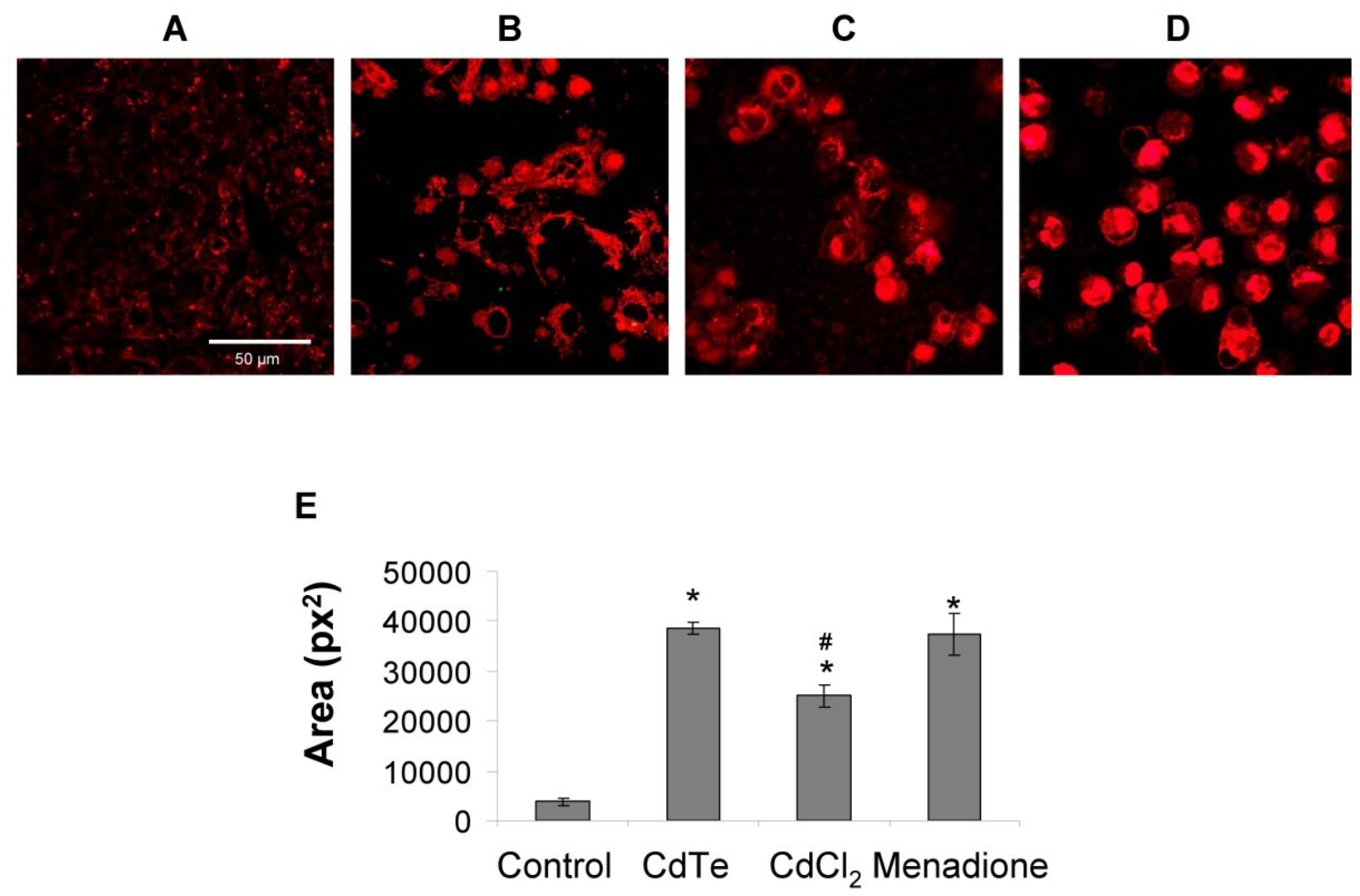

Figure 2.3. Confocal micrographs showing ROS production in HepG2 cells. Representative micrographs of cells treated with (A) PBS, (B) $10 \mu \mathrm{g} / \mathrm{ml}$ CdTe-QDs (containing $1 \mu \mathrm{g} / \mathrm{ml}$ of cadmium) for $24 \mathrm{~h},(\mathbf{C}) 1.63 \mu \mathrm{g} / \mathrm{ml}$ of $\mathrm{CdCl}_{2}$ (containing $1 \mu \mathrm{g} / \mathrm{ml}$ of cadmium), and (D) menadione $(25 \mu \mathrm{M})$, and labeled with DHE. The presence of ROS is indicated with red fluorescence. (E) The total area of red fluorescence (pixels squared: $\mathrm{px}^{2}$ ) was analysed with image analysis software (NIS Elements) using three micrographs for each sample. Each data point represents the mean \pm standard deviation. The asterisks (*) indicate statistically significant differences compared to PBS-treated control $(\mathrm{p}<0.001)$. The number $(\#)$ sign indicates statistically significant differences compared to the CdTe-QD-treated group $(\mathrm{p}<0.05)$. 
A

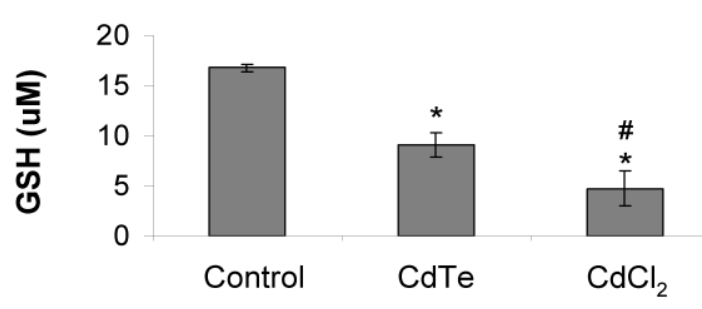

C

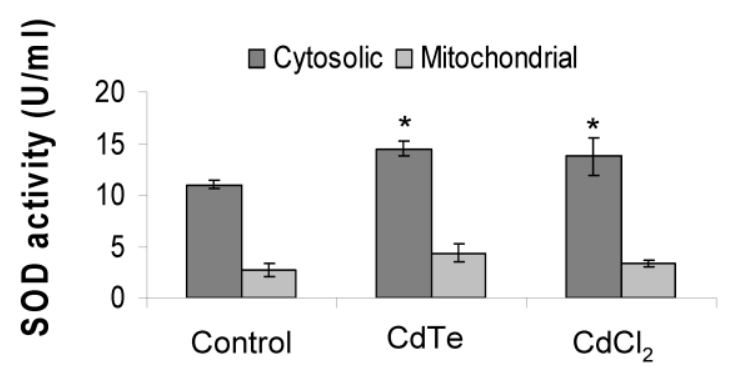

B

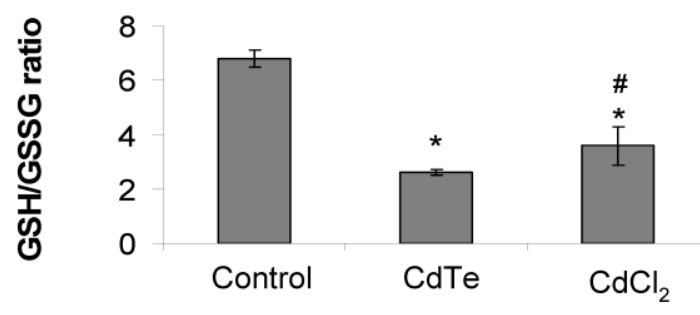

D

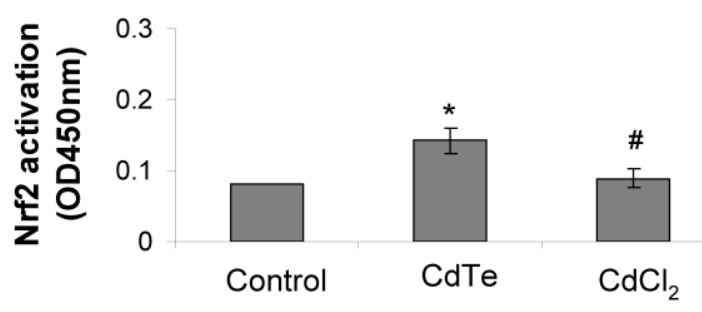

Figure 2.4. Effects of CdTe-QDs on biomarkers of oxidative stress. Cells were treated with $10 \mu \mathrm{g} / \mathrm{ml} \mathrm{CdTe-QDs} \mathrm{(containing} 1 \mu \mathrm{g} / \mathrm{ml}$ of cadmium) or $1.63 \mu \mathrm{g} / \mathrm{ml}$ of $\mathrm{CdCl}_{2}$ (containing $1 \mu \mathrm{g} / \mathrm{ml}$ of cadmium) for $24 \mathrm{~h}$. (A) GSH level as measured using an enzymatic assay, (B) GSH/GSSG ratio, (C) SOD activity as measured using enzymatic assay and (D) Nrf2 activation as measured using ELISA assay and represented by optical density at $450 \mathrm{~nm}\left(\mathrm{OD}_{450}\right) . \mathrm{CdCl}_{2}$ was used for comparison. Data points represent the means of three independent experiments done in duplicate \pm standard deviations. The asterisks $(*)$ indicate statistically significant differences compared to control with $\mathrm{p}<0.001$. The number (\#) signs indicate statistically significant differences compared to the CdTe-QD-treated group $(\mathrm{p}<0.05)$. 


\section{A}

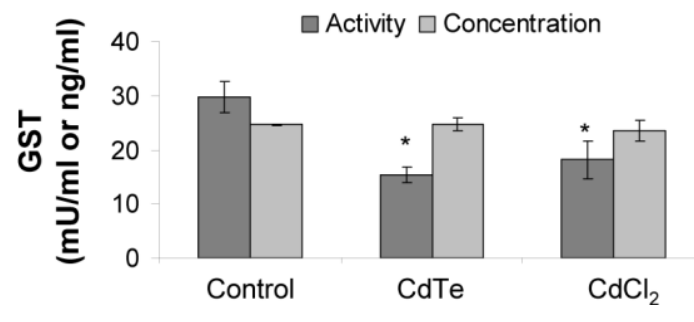

B

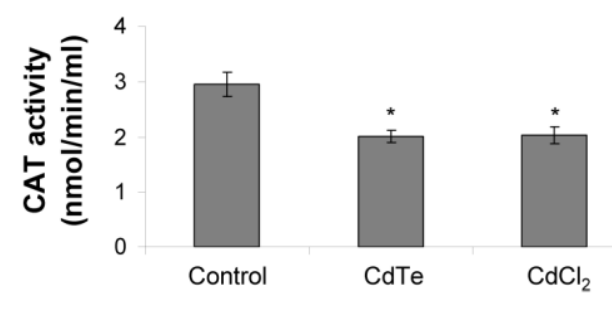

Figure 2.5. Effects of CdTe-QDs on antioxidant enzymes. Cells were treated with 10 $\mu \mathrm{g} / \mathrm{ml} \mathrm{CdTe-QDs} \mathrm{(containing} 1 \mu \mathrm{g} / \mathrm{ml}$ of cadmium) or $1.63 \mu \mathrm{g} / \mathrm{ml}$ of $\mathrm{CdCl}_{2}$ (containing 1 $\mu \mathrm{g} / \mathrm{ml}$ of cadmium) for $24 \mathrm{~h}$. (A) GST activity and concentration as measured using enzymatic and ELISA assays, respectively, (B) CAT activity as measured using enzymatic assay. $\mathrm{CdCl}_{2}$ was used for comparison. Data points represent the means of three independent experiments done in duplicate \pm standard deviations. The asterisks $\left(^{*}\right)$ indicate statistically significant differences compared to PBS-treated control $(p<0.001)$.

\subsubsection{Induction of apoptosis}

As a preliminary screen for apoptosis, caspase-3 activity, level of cleaved PARP and annexin $\mathrm{V}$ binding to externalized phosphatydylserine were examined. CdTe-QDs induced cleavage of pro caspase- 3 to its active form. A 1.6 fold $(p<0.001)$ increase in active form of caspase-3 was observed in CdTe-QD treated cells. $\mathrm{CdCl}_{2}$ and STS treatments also increased caspase-3 activity (Figure 2.6.A). Measurement of cleaved PARP levels in test cells showed that CdTe-QDs induced a significant increase (13.2fold, $\mathrm{p}<0.001)$. While STS treatments also resulted in dramatic increase in PARP cleavage, $\mathrm{CdCl}_{2}$ treatment caused only a moderate elevation $(3.8$-fold, $\mathrm{p}<0.001)$ (Figure 2.6.B). 
A

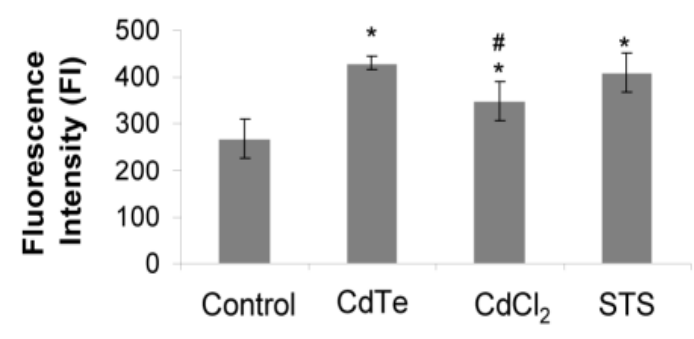

B

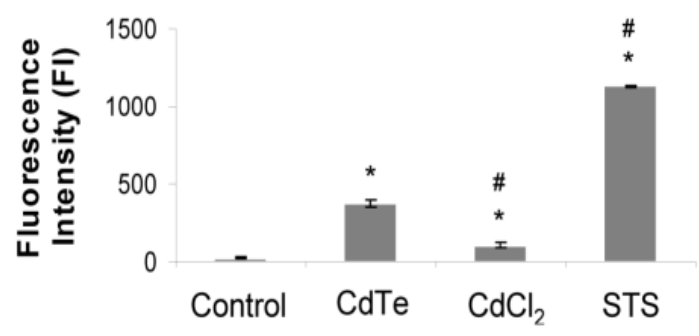

Figure 2.6. Effects of CdTe-QDs on biomarkers of apoptosis. Cells were treated with 10 $\mu \mathrm{g} / \mathrm{ml} \mathrm{CdTe-QDs} \mathrm{(containing} 1 \mu \mathrm{g} / \mathrm{ml}$ of cadmium) or $1.63 \mu \mathrm{g} / \mathrm{ml}$ of $\mathrm{CdCl}_{2}$ (containing 1 $\mu \mathrm{g} / \mathrm{ml}$ of cadmium) for $24 \mathrm{~h}$. (A) Active caspase-3 level and (B) cleaved PARP level were measured using bead plex assays and data were represented by fluorescence intensities (FI). $\mathrm{CdCl}_{2}$ was used for comparison. STS $(1 \mu \mathrm{M})$ was used as a positive control. Data points represent the means of three independent experiments done in duplicate \pm standard deviations. The asterisks $\left(^{*}\right)$ indicate statistically significant differences compared to PBS-treated control $(\mathrm{p}<0.001)$. The number $(\#)$ signs indicate statistically significant differences compared to the CdTe-QD-treated group $(\mathrm{p}<0.05)$.

Cells were treated with conjugated annexin $\mathrm{V}$ and the binding of the protein to externalized phosphatidylserine in apoptotic cells was detected by fluorescence. The results show that while the control cultures had background levels of annexin V staining (Figure 2.7.A), CdTe-QD treatment resulted in a significant increase in annexin $\mathrm{V}$ positive cells (Figure 2.7.B). Both $\mathrm{CdCl}_{2}$ and STS treatments also generated a high number of apoptotic cells that appeared intensely stained with annexin V (Figures 2.7.C and D). 
A
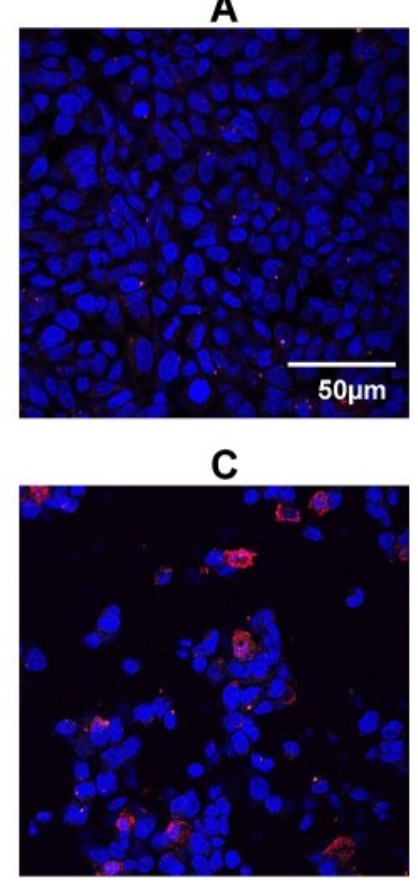

B

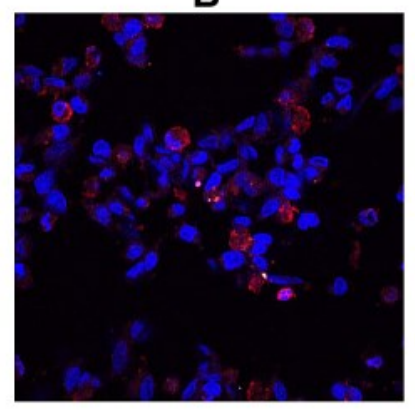

D

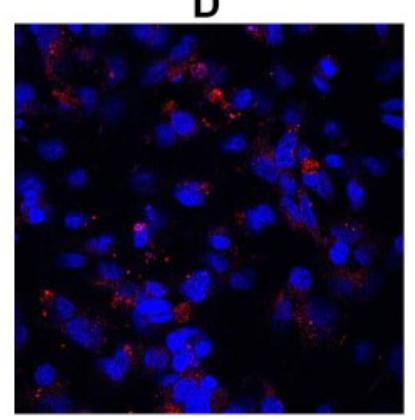

Figure 2.7. Confocal micrographs showing externalization of phosphatidylserine (PS) in HepG2 cells induced by CdTe-QDs. Cells were treated with (A) PBS, (B) $10 \mu \mathrm{g} / \mathrm{ml}$ CdTe-QDs (containing $1 \mu \mathrm{g} / \mathrm{ml}$ of cadmium) for $24 \mathrm{~h}$, (C) $1.63 \mu \mathrm{g} / \mathrm{ml}$ of $\mathrm{CdCl}_{2}$ (containing $1 \mu \mathrm{g} / \mathrm{ml}$ of cadmium), and (D) STS $(1 \mu \mathrm{M})$, and were then stained with annexin V-PE. The binding of annexin V to externalized PS exhibited red fluorescence. Nuclei were stained with Sytox Red ${ }^{\mathrm{TM}}$ and exhibit blue fluorescence.

\subsubsection{Effects of CdTe-QDs on biomarkers of extrinsic apoptotic pathway}

Since Fas-mediated cell death has been suggested to be related to extrinsic apoptosis, the effect of CdTe-QDs on Fas level was examined to reveal details about the apoptotic pathways induced by CdTe-QDs. Treatment of CdTe-QDs induced a marginal increase in Fas level (1.15-fold, $\mathrm{p}<0.05$ ), compared to the control (Figure 2.8.A). While a 
similar effect was observed with $\mathrm{CdCl}_{2}$ treatment, there was no change in Fas level caused by STS (Figure 2.8.A).

Caspase-8 is a marker for extrinsic apoptosis and its activity was examined in HepG2 cells during CdTe-QD exposure. CdTe-QD treatment resulted in increased caspase- 8 activity ( 1.5 fold, $\mathrm{p}<0.001$ ), compared to the control (Figure $2.8 . \mathrm{B}$ ). While $\mathrm{CdCl}_{2}$ treatment also caused increased caspase- 8 activity $(1.2$-fold, $\mathrm{p}<0.001)$, STS treatment caused no change in the activity of this protein (Figure 2.8.B).

A

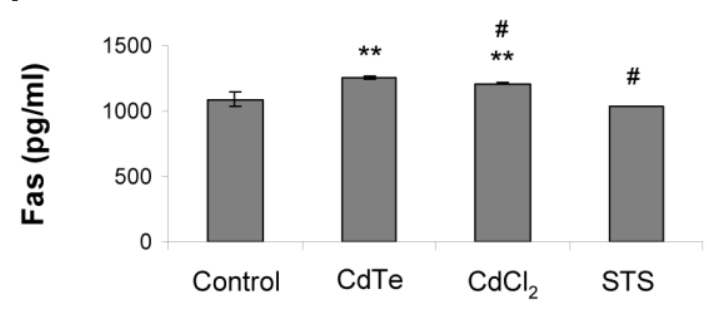

B

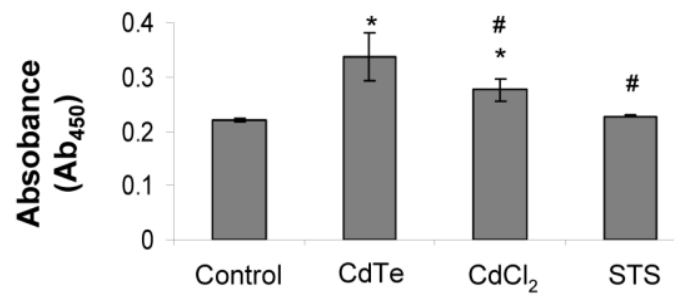

Figure 2.8. Effects of CdTe-QDs on biomarkers of extrinsic apoptosis. Cells were treated with $10 \mu \mathrm{g} / \mathrm{ml} \mathrm{CdTe-QDs} \mathrm{(containing} 1 \mu \mathrm{g} / \mathrm{ml}$ of cadmium) or $1.63 \mu \mathrm{g} / \mathrm{ml}$ of $\mathrm{CdCl}_{2}$ (containing $1 \mu \mathrm{g} / \mathrm{ml}$ of cadmium) for $24 \mathrm{~h}$. (A) Fas level as measured using EIA assay and (B) Caspase 8 activity as measured using enzymatic assay and represented by optical density at $450 \mathrm{~nm}\left(\mathrm{OD}_{450}\right)$. STS $(1 \mu \mathrm{M})$ was used as a positive control. Data points represent the means of three independent experiments done in duplicate \pm standard deviations. The asterisks $(*)$ and $(* *)$ indicate statistically significant differences compared to control with $\mathrm{p}<0.001$ and with $\mathrm{p}<0.05$, respectively. The number (\#) signs indicate statistically significant differences compared to the CdTe-QD-treated group $(\mathrm{p}<0.05)$. 


\subsubsection{Effects of CdTe-QDs on biomarkers of intrinsic apoptotic pathway}

Since Bcl2 is recognized as a potent inhibitor of apoptotic cell death and involved in intrinsic apoptotic pathway, the effect of CdTe-QDs on this protein level in HepG2 was examined. Exposure resulted in a significant decrease in Bcl2 level (1.8-fold, $\mathrm{p}<0.001$ ) (Figure 2.9.A). Similar cell treatments with $\mathrm{CdCl}_{2}$ and STS also led to reduced Bcl2 levels albeit $\sim 10 \%(\mathrm{p}<0.05)$ less than that caused by CdTe-QDs (Figure 2.9.A).

Bax is also an important indicator of intrinsic apoptosis. In this study, Bax levels in both cytosolic and mitochondrial fractions showed that CdTe-QDs induced a significant increase $(1.7$-fold, $\mathrm{p}<0.001)$ in mitochondrial Bax, but resulted in a moderate change in cytosolic Bax (1.2-fold, $\mathrm{p}<0.05)$, compared to the control (Figure 2.9.B). $\mathrm{CdCl}_{2}$ and STS exhibited similar effects as CdTe-QDs (Figure 2.9.B).

Since cytochrome $\mathrm{c}$ is released from mitochondria into cytosol in response to proapoptotic stimuli, its effect during CdTe-QDs exposure was examined. For this, the levels of both cytosolic and mitochondrial cytochrome c during CdTe-QD exposure were compared. Results showed that CdTe-QDs caused reduced mitochondrial cytochrome c level (1.26-fold, $\mathrm{p}<0.001)$, but an increase in cytosolic level (1.26-fold, $\mathrm{p}<0.001)$, compared to the control (Figure 2.9.C). $\mathrm{CdCl}_{2}$ and STS exposures also showed similar effects (Figure 2.9.C). 
A

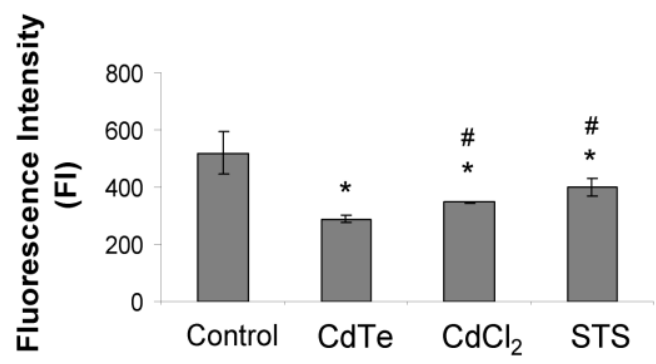

C

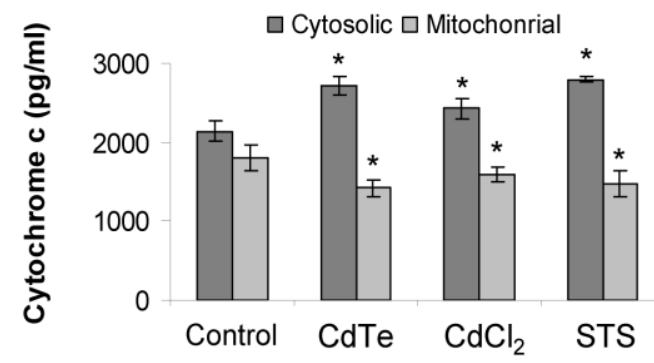

B
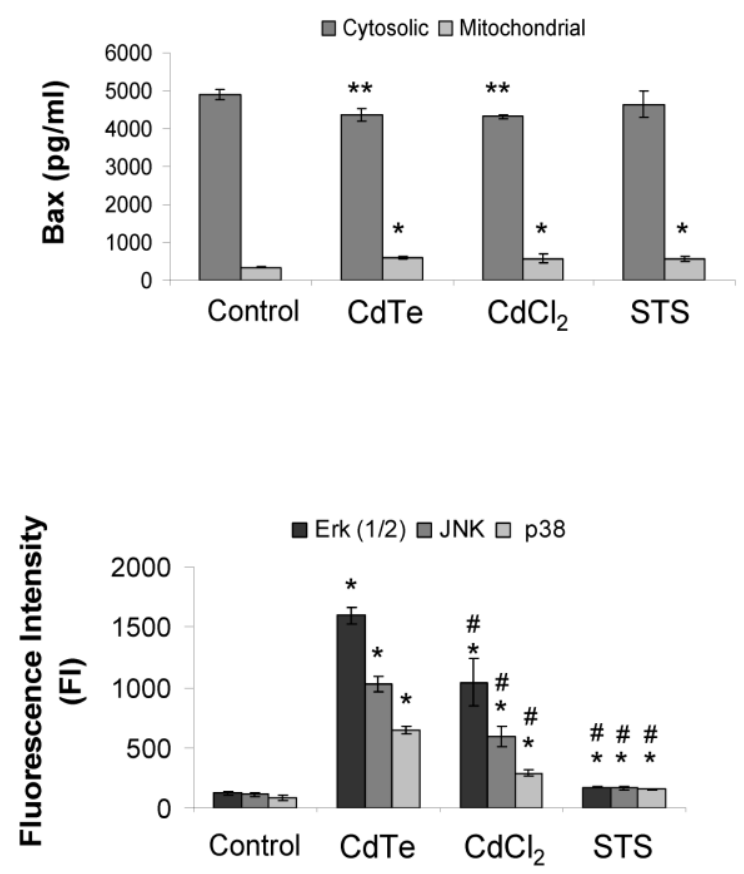

Figure 2.9. Effects of CdTe-QDs on biomarkers of the intrinsic apoptotic pathway and MAPKs. Cells were treated with $10 \mu \mathrm{g} / \mathrm{ml} \mathrm{CdTe-QDs} \mathrm{(containing} 1 \mu \mathrm{g} / \mathrm{ml}$ of cadmium) or $1.63 \mu \mathrm{g} / \mathrm{ml}$ of $\mathrm{CdCl}_{2}$ (containing $1 \mu \mathrm{g} / \mathrm{ml}$ of cadmium) for $24 \mathrm{~h}$. (A) Bcl2 level as measured using bead plex assay and represented by fluorescence intensity (FI), (B) Bax level as measured using EIA assay, (C) Cytosolic and mitochondrial cytochrome $\mathrm{c}$ as measured using ELISA kit and (D) phosphorylated Erk (1/2), JNK and p38 levels as measured using bead plex assay and represented by fluorescence intensity (FI). STS (1 $\mu \mathrm{M})$ was as a positive control. Data points represent the means of three independent experiments done in duplicate \pm standard deviations. The asterisks $(*)$ and $(* *)$ indicate statistically significant differences compared to control with $\mathrm{p}<0.001$ and with $\mathrm{p}<0.05$, respectively. The number (\#) signs indicate statistically significant differences compared to the CdTe-QD-treated group ( $\mathrm{p}<0.05)$. 


\subsubsection{Induction of MAPK activation}

MAPKs such as JNK, p38 and Erk1/2 have been shown to play important roles in apoptotic regulation by way of enzymatic activation through phosphorylation of tyrosine and threonine within their catalytic domains (Wada and Penninger, 2004). Using probes to quantify phosphorylation levels of these MAPKs showed that treatment of CdTe-QDs caused significant increases in levels of phosphorylated JNK, p38 and Erk(1/2) levels (12.8-, 9.0- and 7.5-fold ( $\mathrm{p}<0.001)$, respectively), compared to the control (Figure 2.9.D). Similar treatments with $\mathrm{CdCl}_{2}$ and STS also resulted in significant increases $(\mathrm{p}<0.001)$ in phosphorylation of these MAPKs, compared to the control, but at lower levels, compared to CdTe-QDs $(\mathrm{p}<0.05)$ (Figure 2.9.D).

\subsection{Discussion}

This study presents work using HepG2 cells to model potential mechanisms of hepatocyte toxicity relating to their exposure to CdTe-QDs. Initial results showed that CdTe-QD effects occurred in a dose- and time-dependent manner, consistent with the previous findings using the same source of CdTe-QDs (Nguyen et al., 2013a). While the CdTe-QDs used here are not identical to those used in other studies, the study results are largely consistent with past work using different cell lines including HepG2 (Su et al., 2009; Zhang et al., 2007; Lovric et al., 2005). Su et al. (2009) showed that treatments of 0.1875 to $3 \mu \mathrm{M}$ CdTe-QD to human K562 erythroleukemia and human HEK293T embryonic kidney cells for $30 \mathrm{~min}$ to $48 \mathrm{~h}$ induced changes in bioreduction of MTT in a dose- and time-dependent manner. Similarly, Zhang et al. (2007) reported that treatments of $0-100 \mu \mathrm{M}$ CdTe-QDs for $48 \mathrm{~h}$ to HepG2 cells induced cytotoxicity in a dose- 
dependent manner and proposed that $\mathrm{Cd}^{2+}$ ions were responsible for the cytototoxicity of the NPs. Lovric et al. (2005) also showed that CdTe-QDs caused cytotoxicity in the human breast cancer cell line MCF-7 in a dose-dependent manner after treatment of 1,5, and $10 \mu \mathrm{g} / \mathrm{ml} \mathrm{CdTe}-\mathrm{QDs}$ for $24 \mathrm{~h}$, but the authors claimed that QDs caused cytotoxicity exclusively by inducing ROS formation. These previous studies suggested that toxicity of CdTe-QDs could either be due to the release of $\mathrm{Cd}^{2+}$ ions and/or ROS formation, but more details about possible mechanisms needed to be clarified.

To investigate in greater detail the factors that are possibly responsible for CdTeinduced cytotoxicity, ROS production was measured in situ using the fluorescent dye DHE, which is a specific probe to indicate presence of $\mathrm{O}_{2}^{--}$. Our results showed that CdTe-QD treated cells exhibited an increase in ROS formation, which confirms findings from previous studies that showed ROS generation from CdTe-QD exposures (Lovric et al., 2005; Cho et al., 2007). However many mechanisms can generate ROS by CdTeQDs. The generation of ROS within cells could be directly from the interaction of CdTeQDs with cellular molecules as CdTe-QDs can act as photosensitizers and transfer energy to these molecules (Bakalova et al., 2004). Photolysis or oxidation reactions within the CdTe-QD core may also be a mechanism for ROS production (Lovric et al., 2005). These reactions also produce free $\mathrm{Cd}^{2+}$ ions, which could be another source of ROS production, as cadmium exposure has been previously shown to induce ROS generation in different cell lines (Almazan et al., 2000; Lopez et al., 2006). We used $\mathrm{CdCl}_{2}$ in our study as a control for cadmium-induced effects. Treatment of $\mathrm{HepG} 2$ cells with $\mathrm{CdCl}_{2}$, at an equivalent concentration of cadmium to that contained within CdTe-QDs, also induced elevated ROS compared to controls, but to a lesser extent compared to CdTe-QD 
treatment. Our overall findings suggest that CdTe-QD-induced production of ROS in HepG2 cells is not solely from the effects of cadmium from the QDs, but probably involves other mechanisms.

Excess ROS generation in cells leads to oxidative stress, which in turn induces the action of a cascade of reactive oxygen detoxification systems. If the balance tips in favour of pro-oxidant stress, anti-oxidant defenses become overwhelmed and could result in cell death. In this study, we screened CdTe-QD treated cells with a set of oxidative stress markers. Reduced glutathione (GSH), the most abundant non-protein thiol, has important roles in cellular defense against oxidant aggression from the excess of ROS in cells. Depletion of reduced GSH, which results in a shift in the cellular GSH-to-GSSG redox balance, is considered indicative of oxidative stress (Hug et al., 1994). In this study, the results showed that CdTe-QDs induced a depletion of reduced GSH and a decrease in GSH-to-GSSG ratio, indicating that CdTe-QDs caused oxidative stress in cells. The depletion of GSH could be due to the binding of CdTe-QDs to the thiol group of the protein (Silva et al., 2012). Similarly, cadmium has been shown to bind to the thiol group of GSH causing its depletion (Stohs et al., 2000). CdTe-QDs also resulted in less depletion of reduced GSH compared to $\mathrm{CdCl}_{2}$. This result suggests that, even though both test $\mathrm{CdTe}-\mathrm{QDs}$ and $\mathrm{CdCl}_{2}$ contain an equivalent amount of cadmium and if there is any free $\mathrm{Cd}^{2+}$ released from CdTe-QDs, the level of free $\mathrm{Cd}^{2+}$ released from CdTe-QDs in test cells was much less, resulting in less consumption of GSH thiol groups.

Other oxidative stress biomarkers that were measured were SOD activity and Nrf2 activation. SOD catalyzes the dismutation of superoxide into oxygen and hydrogen peroxide. Measurement of SOD activity is an indirect method of detecting ROS, since 
SOD activity reflects superoxide production in cells. Nrf2 is a transcription factor that is activated during oxidative stress and translocated from the cytoplasm to the nucleus to bind the antioxidant response element (ARE), activating transcription of antioxidant and xenobiotic response genes (Venugopal et al., 1996). The increase in SOD activity and Nrf2 activation in this study confirmed that oxidative stress was caused by CdTe-QD exposure. Compared to $\mathrm{CdCl}_{2}, \mathrm{CdTe}-\mathrm{QDs}$ caused greater oxidative stress as demonstrated by a lower GSH/GSSG ratio, increased SOD activity and Nrf2 activation, suggesting that cadmium from CdTe-QDs cannot account for the entire effect. However, other factors such as the intrinsic nanoscale properties of QDs and ROS generated from the NPs may contribute to the observed oxidative stress.

The glutathione S-transferases (GSTs) are a family of antioxidant enzymes important for detoxification of xenobiotics and peroxidation products (Hayes and McLellan, 1999). Under oxidative stress, GST activity is expected to increase from elevated levels of organic and non-organic peroxides, which act as substrates for the enzyme (Hayes and McLellan, 1999). Treatment of HepG2 cells with CdTe-QDs resulted in decreased GST activity, but unchanged GST protein abundance. This revealed that decreased GST activity was not a result of cell death and that CdTe-QDs might have an inhibitory action on GST itself.

Similar to GST, CAT, which is an antioxidant, catalyzes the decomposition of $\mathrm{H}_{2} \mathrm{O}_{2}$ to oxygen and water and is well known as an important antioxidant enzyme (Chelikani et al., 2004). Treatment of HepG2 cells with CdTe-QDs resulted in decreased CAT activity. Although CAT protein level was not measured in this study, lowered CAT activity was probably also related to the activity inhibition of CdTe-QDs, but not from 
cell death. Cadmium has previously been reported to inhibit GST and CAT activity in vitro and in vivo (Dierickx, 1982; Pruell and Engelhardt, 1980). By inhibiting GST and CAT activities, CdTe-QDs appear to impair cellular antioxidant defenses, leading to oxidative stress. The inhibition of the activities of these antioxidant enzymes by CdTeQDs suggests that cadmium might have a role in the effects of these NPs.

Oxidative stress is an important factor for triggering apoptosis (Buttke and Sandstrom, 1994). Our results showed that CdTe-QDs induced an increase in certain apoptotic hallmarks. Caspase-3 is a protease catalyzing the specific cleavage of many key cellular proteins. The increase in caspase-3 activity was confirmed with the increased cleavage of PARP, the action of which is catalyzed by the protease caspase-3. Furthermore, apoptosis was confirmed with the observation of annexin $\mathrm{V}$ binding to externalized phosphatidylserine, an event typical of apoptosis.

Since caspase-3 activation is a central feature of apoptotic cells, further investigation of the pathways involving CdTe-QD-induced apoptosis was done. Our results showed that CdTe-QDs resulted in an increase in Fas level and in caspase-8 activity indicating that CdTe-QDs cause apoptosis in HepG2 cells via extrinsic pathways. Our findings align with the previous study by Choi and colleagues (2007) who reported that CdTe-QD treatment to human neuroblastoma cells caused apoptosis associated with increased Fas expression (Choi et al, 2007). Our results also showed that exposure to CdTe-QDs caused effects on anti-apoptotic protein Bc12 and pro-apoptotic protein Bax levels, which are biomarkers for the intrinsic pathway (Dejean et al, 2006). Bcl2 is a key inhibitor of apoptosis since its over-expression blocks translocation of cytochrome c from mitochondria into cytosol, thus preventing cells from undergoing apoptosis (Yang et al., 
1997). Conversely, over-expression of Bax and its translocation to mitochondria have been shown to promote the release of cytochrome $\mathrm{c}$ into cytosol, leading to activation of effector caspases and subsequently to apoptosis (Finucane et al., 1999). Our results showed CdTe-QDs induced a decrease in Bcl2 and an increase in mitochondrial Bax levels, suggesting intrinsic apoptotic pathway induction. The release of cytochrome c from mitochondria to cytosol confirmed the effects on cellular Bcl2 protein members.

In addition, the present study also revealed that CdTe-QDs activated MAPK signalling pathways as indicated by increases in phosphorylation levels of JNK and p38. These results are supported by a previous study reporting that activation of JNK and p38 led to phosphorylation and translocation of Bax into mitochondria to cause apoptosis via intrinsic pathway (Kim et al., 2006). The present study also adds to the findings from the previous studies by Lu et al. (2006) who showed that activation of JNK was required for CdSe-QDs induced apoptosis in human osteoblast cells. Similarly, Chan et al. (2006) reported that CdSe-QDs induced apoptosis in human neuroblasma cells via intrinsic (mitochondrial) pathway involving JNK activation. However, contrary to the report from Chan and colleagues who showed that their test QDs caused a decrease in Erk level resulting in inhibition of Ras to Erk survival signalling (Chan et al., 2006), the present study observed an increase in Erk1/2. The reason for the difference between these findings might be due to differences in the test cell lines, as suggested in the existing literature that Cd-induced Erk activation is cell type-dependent (Martin and Pognonec, 2010).

Cadmium has also been shown to cause apoptosis in vitro and in vivo and the apoptosis induced by cadmium is suggested to arise from the effects of ROS generated by 
the metal (Hamada et al., 1997; Oh and Lim, 2006). The cadmium-induced apoptotic pathway has been suggested to be dependent on test models and conditions as several studies on cadmium-induced apoptosis yielded different outcomes (Kondoh et al., 2002; Shih et al., 2004; Li and Lim, 2007). In HepG2, cadmium has been shown to cause apoptosis via both extrinsic and intrinsic pathways (Oh and Lim, 2006). Similarly, ROS alone have also been shown to cause apoptosis via both pathways (Simon et al., 2000). In this study, $\mathrm{CdCl}_{2}$ was also shown to cause similar effects on the apoptotic biomarkers of both pathways, but the effects were less pronounced compared to that of CdTe-QDs, suggesting that the effects of CdTe-QDs possibly involve both cadmium and ROS generated from these NPs. Our findings support the suggestions from recent studies on the mechanisms of cadmium-based QD-induced toxicity in different cell lines and in an invertebrate model organism that QD treatments resulted in more severe toxic effects than cadmium at the same concentration, suggesting that the QD effects were not only from the release of $\mathrm{Cd}^{2+}$ ions but also from the properties of the NPs and ROS generated from them (Li et al., 2009; Chen et al., 2012; Ambosone et al., 2012).

In conclusion, the present study investigated the mechanism of toxic effects caused by CdTe-QDs in HepG2 cells and revealed that CdTe-QDs caused cytotoxicity in these cells by inducing oxidative stress leading to apoptosis. Oxidative stress induced by CdTe-QDs was evidenced by the increase in ROS production and the interference of these NPs on the antioxidant defenses in test cells. CdTe-QDs caused apoptosis in test cells via both extrinsic and intrinsic pathways. Even though the release of $\mathrm{Cd}^{2+}$ from CdTe-QDs was not measured in this study, treatments of cells with equivalent cadmium concentrations (in the form of $\mathrm{CdCl}_{2}$ ) were conducted for comparative purposes. Since 
the effects of Cd-QDs appeared similar or greater to those of $\mathrm{CdCl}_{2}$, it was postulated that the toxicity of CdTe-QDs arises from more than one factor, including cadmium effects, ROS generation and the intrinsic nano-scale properties of CdTe-QDs. The study provides valuable information for understanding the toxicity of CdTe-QDs which is important for safety evaluation of the nanoparticles for future biomedical applications.

Further studies are needed to define the sources of ROS generation and oxidative stress induced by CdTe-QDs in the test cells. For this purpose, Chapter 3 of this research focused the effects of these NPs on mitochondria to elucidate the detailed mechanisms that led to cytotoxicy in HepG2 observed in Chapter 2. 


\section{Chapter: Mitochondrial Toxicity of Cadmium Telluride Quantum Dot Nanoparticles in Mammalian Hepatocytes}

This Chapter is adapted from the following article:

Kathy C Nguyen, Peter Rippstein, Azam F Tayabali, and William G Willmore. 2015. Mitochondrial Toxicity of Cadmium Telluride Quantum Dot Nanoparticles in Mammalian Hepatocytes. Toxicological Sciences. 146(1): 31-42.

Permission was obtained from the Toxicological Sciences journal for incorporation of this paper into this thesis. Minor changes were performed to the formatting of the documemt so that it could fit in the format of this dissertation.

For this paper, Kathy C. Nguyen designed and conducted these experiments and wrote the manuscript. Peter Ripstein (Heart Institute, University of Ottawa) performed TEM work. Drs. William Willmore and Azam Tayabali provided input into the manuscript. Dr. Azam Tayabali and Health Canada provided funding for the project. Dr. William Willmore provided funding for coloured figures in print versions of the paper. 


\subsection{Abstract}

There are an increasing number of studies indicating that mitochondria are relevant targets in nanomaterial-induced toxicity. However, the underlying mechanisms by which nanoparticles interact with these organelles and affect their functions are unknown. The aim of this study was to investigate the effects of cadmium telluride quantum dot (CdTe-QD) nanoparticles (NPs) on mitochondria in human hepatocellular carcinoma HepG2 cells. CdTe-QD treatment resulted in the enlargement of mitochondria as examined with transmission electron microscopy and confocal microscopy. CdTeQDs appeared to associate with the isolated mitochondria as detected by their inherent fluorescence. Further analyses revealed that CdTe-QD caused disruption of mitochondrial membrane potential, increase in intracellular calcium levels, impaired cellular respiration, and decreased ATP synthesis. The effects of CdTe-QDs on mitochondrial oxidative phosphorylation were evidenced by changes in levels and activities of the enzymes of the electron transport chain. Elevation of peroxisome proliferator-activated receptor- $\gamma$ coactivator $(\mathrm{PGC}-1 \alpha)$ levels after CdTe-QD treatment suggested effects of CdTe-QDs on mitochondrial biogenesis. Our results also showed that the effects of CdTe-QDs were similar or greater to those of $\mathrm{CdCl}_{2}$ at equivalent concentrations of cadmium, suggesting that the toxic effects of CdTe-QDs were not solely due to cadmium released from the NPs. Overall, the study demonstrated that CdTe-QDs induced multifarious toxicity by causing changes in mitochondrial morphology and structure, as well as impairing their function and stimulating their biogenesis. 


\subsection{Introduction}

Nanotechnology has potential applications in a wide variety of fields including medicine, cosmetics, electronics, textiles, and engineering. While the use of nanomaterials is growing rapidly, there is increasing concern that these materials might pose potential risks to human health (Dreher, 2004; Xia et al., 2010). Quantum dots (QDs) are engineered nanoparticles (NPs) and possess unique optical and electronic properties that make them useful materials in microelectronics and biomedical research (De Wild et al., 2003). Cadmium quantum dots including cadmium telluride quantum dots (CdTe-QDs) and cadmium selenide quantum dots (CdSe-QDs) have shown great potential for use as fluorescent tags in therapeutic targeting and in medical and molecular imaging (Chan et al., 2002; Gao et al., 2004; Zhu et al.,2013). While the applications of these QDs are growing, there are concerns about their potential hazards to human health and the environment. However, the toxicity associated with these NPs and the underlying mechanisms have not been thoroughly evaluated.

The mitochondrion is one of the most important organelles in cells as it plays a critical role in cellular energy production. Adenosine triphosphate (ATP) production by mitochondria is achieved through oxidative phosphorylation involving the electron transport chain (ETC), the proteins of which are located in the inner membrane of mitochondria. Besides energy production, other crucial roles of mitochondria include the maintenance of calcium homeostasis, intermediate metabolism, the generation of reactive oxygen species (ROS), and the initiation or progression of programmed cell death (Zorov et al., 1997). Mitochondrial damage could result in changes in cell functions that inevitably lead to disease states. Diseases in which mitochondrial dysfunction have been 
identified as being the causative factor include metabolic syndrome, cancer, diabetes, cardiovascular diseases, and several neurodegenerative diseases (Wojtczak and Zabłocki, 2008).

Several studies have suggested that mitochondria are potentially relevant target organelles for NP toxicity (Zhu et al., 2006; Oberdoster et al., 2005; and Xia et al., 2006). A study by Li and colleagues (2003) found that ultrafine particulate pollutants entered the mitochondria and induced mitochondrial damage and cellular vacuole formation. Similarly, Xia's group (2006) showed that diesel exhaust particles (DEP) caused morphological alterations in mitochondria, including swelling and loss of cristae, in human lung epithelial cells (BEAS-2B). Furthermore, a study on various test metal NPs, including silver, iron oxide, aluminum, molybdenum oxide, and titanium dioxide, showed that these NPs induced changes in mitochondrial membrane potential (Hussain et al., 2005). More recently, several studies have shown the effects of NPs, including copper oxide, silica, iron oxide, and fullerene NPs, on mitochondrial membrane potential and respiratory chain complexes activities in different cell types and isolated mitochondria from rat organs (Sun et al., 2011; Xue et al., 2014; Siddiqui et al., 2013; Baratli et al., 2013; Santos et al., 2014). However, there is little known information on how NPs interact with mitochondria and affect their function.

This study aimed to investigate mitochondrial changes induced by CdTe-QDs. A human hepatocellular liver carcinoma cell line (HepG2) was used as an in vitro model to examine CdTe-QD toxicity. HepG2 is a relevant in vitro model for studying toxicity as the cell line retains many functions of human liver cells (Camp and Capitano, 2007). These cells are also an excellent model to investigate mitochondrial toxicity because of 
their high content of organelles and mitochondrial DNA (mtDNA) (Pinti et al., 2003). Our previous studies have demonstrated the toxicity of CdTe-QDs in HepG2 and other cell lines (Nguyen et al., 2013a; Nguyen et al., 2013b). In Chapter 2 of this thesis, CdTeQDs have been shown to cause cytotoxicity and oxidative stress leading to extrinsic and intrinsic apoptosis (Nguyen et al., 2013b). Since mitochondria have been linked to oxidative stress and apoptotic pathways, studying the effects of CdTe-QDs on mitochondria is an important extension of toxicity investigation to reveal the details of the effects induced by these NPs in test cells that were observed in our previous studies. In this study, a set of cellular assays were used to examine the effects of CdTe-QDs on mitochondrial structure and function including mitochondrial morphology, membrane potential, calcium regulation, ATP production, cellular respiration, electron transport chain or oxidative phosphorylation coupling. The effect of CdTe-QDs on mitochondrial biogenesis was also investigated. CdTe-QDs were compared with those of $\mathrm{CdCl}_{2}$ with equivalent concentrations of cadmium. Elucidating the effects of CdTe-QDs on mitochondria will further our understanding of the intracellular behavior of these NPs within target cells as well as their mechanisms of toxicity. These results will ultimately add to our understanding of potential human health issues and introduce cellular biomarkers that may serve to warn against adverse effects of NPs.

\subsection{Materials and Methods}

\subsubsection{Chemicals and reagents}

HepG2 cells were obtained from ATCC (Manassas, VA). CdTe-QDs were purchased from Nano Impex Canada (Mississauga, ON). CdTe-QDs were described by 
the manufacturer as $\mathrm{CdTe} / \mathrm{CdS}$ core/shell QDs, encapsulated by polyacrylate polymer layers, with a size of $5 \mathrm{~nm}$, a spectral emission of $540 \mathrm{~nm}$ and a concentration of 10 $\mathrm{mg} / \mathrm{ml}$ in water, $4.9 \times 10^{13} \mathrm{particles} / \mathrm{ml}$, and containing $10 \%$ of cadmium. MTT [3-(4,5dimethylthiazol-2-yl)-2,5-diphenyl tetrazolium bromide], dimethyl sulfoxide (DMSO), cadmium chloride $\left(\mathrm{CdCl}_{2}\right)$, and sodium tellurite $\left(\mathrm{Na}_{2} \mathrm{TeO}_{3}\right)$ were obtained from SigmaAldrich (St. Louis, MO). Eagle's Minimum Essential Medium (EMEM), fetal bovine serum (FBS), gentamicin, phosphate saline buffer (PBS), and calcium crimson were obtained from Life Technologies (Carlsbad, CA). Tris base, 3-(N-morpholino) propanesulfonic acid (MOPS), sucrose, ethylene glycol tetraacetic acid (EGTA), rotenone, succinate, glutamate, malate, malonate, sodium cyanide $(\mathrm{NaCN})$, and adenosine diphosphate (ADP) were purchased from Sigma-Aldrich (St. Louis, MO). Glutaraldehyde, osmium tetroxide, and sodium cacodylate were purchased from Electron Microscopy Science (Hatfield, PA).

\subsubsection{Characterization of CdTe-QDs}

The spectral properties and size characterization of test CdTe-QDs were carried out as described in Chapter 2.

Inductively coupled plasma optical emission spectrometry (ICP-OES) analysis was used to determine the elemental concentrations in CdTe-QDs. First, CdTe-QD sample $(2.5 \mathrm{mg} / \mathrm{ml})$ was prepared in liquid chromatography/mass spectrometry (LC/MS) grade water containing $5 \% \mathrm{HNO}_{3}$. The metal composition of particles was analyzed by ICP-OES using a Perkin Elmer Optima 5300DV Emission Spectrometer (Waltham, MA) with axial viewing and modified background correction. Elements of interest were 
confirmed using a minimum of two analytical wavelengths for each element. Five replicate readings were performed on each analyte.

\subsubsection{Cell preparation and treatment with CdTe-QDs}

HepG2 cells were cultured in Eagle's minimum essential medium (EMEM) supplemented with $10 \% \mathrm{FBS}$ and $50 \mu \mathrm{g} / \mathrm{ml}$ gentamicin in a humidified tissue culture incubator at $37^{\circ} \mathrm{C}$ with $5 \% \mathrm{CO}_{2}$. For the MTT assay, cells were seeded into 96-well plates at density of $5 \times 10^{4}$ cells $/ 100 \mu \mathrm{L}$ well. For TEM, confocal microscopy (including Mitotracker ${ }^{\circledR}$, membrane potential, and calcium detection), ATP detection, mitochondrial complex quantification, or PGC-1 $\alpha$ detection assay, cells were seeded on cover-slips placed in 6-well plates at a concentration of $2 \times 10^{5}$ cells per well in $2 \mathrm{ml}$ of complete medium. For mitochondrial isolation or mitochondrial complex activity assay, cells were seeded on $75 \mathrm{~cm}^{2}$ culture flasks at a concentration of $1 \times 10^{6}$ cells per flask. Cells were cultured for $24 \mathrm{~h}$ to $80 \%$ confluency and media was replaced before the exposures. Working solutions of CdTe-QDs and $\mathrm{CdCl}_{2}$ were prepared by diluting the stock solutions in phosphate-buffered saline (PBS). For the MTT assay, cells were treated with different concentrations of CdTe-QDs and $\mathrm{CdCl}_{2}$ (containing $0.01 \mu \mathrm{g} / \mathrm{ml}$ to $1 \mu \mathrm{g} / \mathrm{ml}$ of cadmium) for different durations. Cells were also treated with different concentrations of $\mathrm{Na}_{2} \mathrm{TeO}_{3}$ (containing 0.00033 to $0.33 \mu \mathrm{g} / \mathrm{ml}$ of tellurium) for the same durations. The concentrations of tellurium were based on the mass ratio of tellurium:cadmium in CdTeQDs, which is $0.33: 1$, based on the ICP-MS results. To elicit mitochondrial effects, for other assays, cells were treated with $10 \mu \mathrm{g} / \mathrm{ml}$ (or $4.9 \times 10^{5}$ particles/cell) CdTe-QDs (containing $1 \mu \mathrm{g} / \mathrm{ml}$ of cadmium) and $1.63 \mu \mathrm{g} / \mathrm{ml}$ of $\mathrm{CdCl}_{2}$ (containing $1 \mu \mathrm{g} / \mathrm{ml}$ of 
cadmium) for $24 \mathrm{~h}$. Treatments with PBS were used as negative controls. For ATP, ETC concentration and activity, and PCG- $\alpha$ assays, the same protein concentrations of the control and treated samples were used. CdTe-QDs alone were tested with reagents of the ATP and PCG- $\alpha$ assays to examine the interference of the NPs with the assay systems. All measurements were conducted in duplicate in three independent experiments.

\subsubsection{Cytotoxicity using the MTT assay}

The MTT assay was conducted as described in Nguyen et al. (2013a). Briefly, following the treatment of cells with $\mathrm{CdTe}-\mathrm{QDs}, \mathrm{CdCl}_{2}$, or $\mathrm{Na}_{2} \mathrm{TeO}_{3}$, medium was removed and replaced with fresh medium (100 $\mu \mathrm{l} /$ well). A total of $10 \mu \mathrm{l}$ stock MTT (10 $\mathrm{mg} / \mathrm{ml}$ ) was added to each well and cells were incubated for $1 \mathrm{~h}$ at $37^{\circ} \mathrm{C}$. Media was removed and cells were rinsed with PBS (100 $\mu 1 /$ well). Cells were lysed and formazan was solubilized with DMSO $(100 \mu \mathrm{l} /$ well $)$. Absorbance was measured at $505 \mathrm{~nm}$ using a multiwall scanning spectrophotometer (Molecular Devices, Sunnyvale, CA).

\subsubsection{TEM for mitochondrial morphology}

HepG2 cells were treated with CdTe-QDs $(10 \mu \mathrm{g} / \mathrm{ml}$ containing $1 \mu \mathrm{g} / \mathrm{ml}$ of cadmium) for $24 \mathrm{~h}$. Cells were then fixed with $2.5 \%$ glutaraldehyde in $66.7 \mathrm{mM}$ cacodylate buffer ( $\mathrm{pH}$ 7.4) for $1 \mathrm{~h}$, washed twice with $100 \mathrm{mM}$ cacodylate buffer for 10 min, and then processed with standard procedures. Post fixation was done with $1 \%$ osmium tetroxide in $100 \mathrm{mM}$ cacodylate buffer, $\mathrm{pH}$ 7.4. The monolayers of cells were dried through a series of alcohols, infiltrated with epoxy resin, and embedded onto resinfilled Beem ${ }^{\circledR}$ capsule molds. The blocks were then ultrathin-sectioned and stained first 
with uranyl acetate and then with lead citrate, and analysed with a Joel 1230 transmission electron microscope.

\subsubsection{Mitochondrial isolation}

Mitochondria from HepG2 cells were isolated as previously described by Frezza et al, 2007 with minor modifications. Briefly, cells were washed with PBS and collected into a $50 \mathrm{ml}$ tube. The tube was centrifuged at $1,000 \mathrm{xg}$ for $5 \mathrm{~min}$ at $4^{\circ} \mathrm{C}$. The cell pellet then was resuspended in $3 \mathrm{ml}$ of isolation buffer (10 mM Tris-MOPS, $100 \mathrm{mM}$ sucrose, and $1 \mathrm{mM} \mathrm{EGTA/Tris),} \mathrm{transferred} \mathrm{to} \mathrm{a} \mathrm{homogenizing} \mathrm{glass} \mathrm{tube} \mathrm{and} \mathrm{homogenized} \mathrm{at}$ 1,400 rpm with 40 strokes using an IKA Eurostar power control-visc stirrer $\left(\right.$ IKA $^{\circledR}$ works, Inc., Wilmington, NC). The sample was then centrifuged at $600 \mathrm{xg}$ for $10 \mathrm{~min}$ at $4^{\circ} \mathrm{C}$. The collected supernatant was then centrifuged at $10,000 \mathrm{x} \mathrm{g}$ for $10 \mathrm{~min}$ at $4^{\circ} \mathrm{C}$. The pellet containing mitochondria was resuspended in isolation buffer on ice and protein concentration was determined using BCA kit (Pierce, Rockford, IL).

\subsubsection{Determination of CdTe-QDs in mitochondrial enrichment}

Isolated mitochondrial samples $(100 \mu \mathrm{l})$ from PBS and CdTe-QD treated cells were added to a 96-well opaque plate and fluorescence was measured at excitation of 485 $\mathrm{nm}$ and emission of $540 \mathrm{~nm}$ with a SpectraMax GEMINI XS microplate spectrofluorometer (Molecular Devices, Sunnyvale, CA).

\subsubsection{Mitochondrial membrane potential measurements by confocal microscopy}


Cells were grown to $80 \%$ confluency on glass cover slips placed inside 6-well tissue culture grade plates. After treatment with either CdTe-QDs or $\mathrm{CdCl}_{2}$ for $24 \mathrm{~h}$ or carbonylcyanide m-chlorophenylhydrazone (CCCP) (as a positive control) for $1 \mathrm{~h}$, cells were washed and loaded with $100 \mathrm{nM}$ of the fluorescent potential-dependent indicator, tetramethylrhodamine, ethyl ester (TMRE) (Immunochemistry Technologies LLC, Bloomington, $\mathrm{MN}$ ) for $30 \mathrm{~min}$ at $37^{\circ} \mathrm{C}$. Cells were washed with assay buffer and observed with a Nikon TE2000 microscope attached to a C1 confocal unit.

\subsubsection{Calcium level detection}

Cells were grown to $80 \%$ confluency on glass cover-slips inside 6-well tissue culture grade plates. After treatment with CdTe-QDs or $\mathrm{CdCl}_{2}$ for $24 \mathrm{~h}$, cells were washed and treated with calcium crimson at a concentration of $10 \mu \mathrm{M}$ for $20 \mathrm{~min}$. Cells were then washed with PBS and the cover-slip containing the monolayer of cells was mounted on a slide and viewed immediately with a Nikon TE2000 microscope attached to a $\mathrm{C} 1$ confocal unit.

\subsubsection{Mitochondrial complex quantification assay}

The mitochondrial complex levels were measured using a BeadPlex ${ }^{\mathrm{TM}}$ mitochondrial oxidative phosphorylation kit from EMD (Gibbstown, NJ), according to the manufacturer's instructions. Briefly, after treatment, cells were rinsed with ice-cold PBS and then homogenized in supplied lysis buffer containing protease inhibitors. The cell lysates were centrifuged at $12,000 \mathrm{rpm}$ for $20 \mathrm{~min}$ at $4^{\circ} \mathrm{C}$ and the supernatants were collected and diluted in assay buffer. Each standard or sample (50 $\mu 1)$ was added to the 
corresponding well in a filter 96-well plate containing $25 \mu 1$ of anti-complex conjugated beads and $25 \mu \mathrm{l}$ of detection antibody. The plate was incubated for $3 \mathrm{~h}$ on a shaker at RT then washed three times with wash buffer before $50 \mu 1 /$ well of pre-diluted streptavidinconjugated phycoerythrin was added. The plate was incubated for $30 \mathrm{~min}$ on a shaker at RT. After being washed, $120 \mu \mathrm{l}$ of wash buffer was added to each well. The plate was shaken for 1 min and analyzed with the Bio-Plex 100 Array System (Bio-Rad, Hercules, CA).

\subsubsection{Mitochondrial complex activity assay}

Mitochondrial Complexes I, II, III, IV, and V activities were measured using enzyme activity ELISA and MitoTox OXPHOS activity assay kits from Mitosciences (Eugene, OR) following the manufacturer's instructions with minor modifications. For Complexes I, II, IV, and V, control and CdTe-QD or $\mathrm{CdCl}_{2}$ treated cells were first lysed in lysis buffer containing detergent and incubated on ice for $30 \mathrm{~min}$. The samples were centrifuged at $16,000 \mathrm{rpm}$ for $20 \mathrm{~min}$ at $4^{\circ} \mathrm{C}$ and supernatants were collected. Samples (200 $\mu 1$ for Complexes I and IV, $50 \mu 1$ for Complexes II and V) were added to assigned wells in the microplate provided for each corresponding assay. The plate was incubated for $3 \mathrm{~h}$ at RT. After washing, $200 \mu \mathrm{l}$ of substrate solution was added to each well, except in the assays for Complexes II and V, lipid mix (40 $\mu \mathrm{l})$ was added to each well and the plate was incubated for $45 \mathrm{~min}$ at RT, before adding substrate solution. The plate was placed immediately in a microplate reader and the absorbance was measured in kinetic mode at 450, 600, 550 or $340 \mathrm{~nm}$ for Complexes I, II, IV, or V respectively. 
For Complex III, the activity of the complex was performed using untreated isolated mitochondria of HepG2 cells. Briefly, $10 \mu 1$ of CdTe-QDs or $\mathrm{CdCl}_{2}$ (containing $1 \mu \mathrm{g} / \mathrm{ml}$ of cadmium) was added to each assigned well of a 96-well plate containing 100

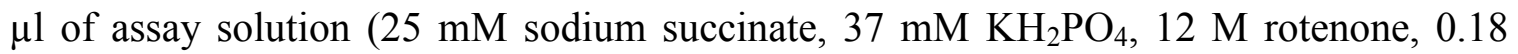
$\mathrm{mM} \mathrm{NaCN}$, and $200 \mathrm{M}$ oxidized cytochrome c). Untreated isolated mitochondria solution $(20 \mu \mathrm{l})$ was added to each well and the plate was placed immediately in a microplate reader and the absorbance was measured in kinetic mode at $550 \mathrm{~nm}$.

\subsubsection{Cellular respiration assay}

Oxygen consumption was monitored in 96-well plate format using a phosphorescent oxygen sensitive probe as previously described (Hynes et al., 2006) with minor modifications. Briefly, oxygen probe (Luxcel Biosciences, Cork, Ireland) was reconstituted in $10 \mathrm{ml}$ of respiratory buffer to a concentration of approximately $100 \mathrm{nM}$. To each well of a 96 well plate, $100 \mu 1$ of this solution was added. CdTe-QDs $(1 \mu 1$ well, $10 \mu \mathrm{g} / \mathrm{ml}$ or containing $1 \mu \mathrm{g} / \mathrm{ml}$ of cadmium), rotenone (Complex I inhibitor), or malonate (Complex II inhibitor) was added to the assigned wells. After that, $50 \mu 1$ of mitochondrial stock solution $(1.0 \mathrm{mg} / \mathrm{ml})$ was added to each well followed by $50 \mu \mathrm{l}$ of a $50 / 50 \mathrm{mM}$ glutamate/malate mixture or $100 \mathrm{mM}$ succinate without or with ADP (6.6 $\mathrm{mM}$ final concentration) in incubation buffer. Finally, $100 \mu \mathrm{l}$ of heavy mineral oil was added to each well and the plate was placed in a SpectroMax Gemini (Molecular Devices), equilibrated at $30^{\circ} \mathrm{C}$, and measured kinetically at $380 / 650 \mathrm{~nm}$ excitation/emission every $5 \mathrm{~min}$ over a period of $120 \mathrm{~min}$. Time profiles of fluorescence intensity in each well were analyzed using Excel (Microsoft) software, to determine the 
rates of oxygen consumption based on the known relationship between probe fluorescence and oxygen concentration (Hynes et al., 2006). Rates of change of dissolved oxygen were subsequently determined from the slopes of these concentration profiles.

\subsubsection{ATP level detection}

ATP quantification was carried out using ATP colorimetric assay kit from Biovision (Mountain View, CA). Cells were lysed in ATP assay buffer. The samples were centrifuged at $15,000 \mathrm{~g}$ for $2 \mathrm{~min}$ at $4^{\circ} \mathrm{C}$ and the supernatants were collected for the assay. Samples were diluted with ATP buffer and added to the corresponding wells on a microplate. CdTe-QDs alone were added to separate wells to test for the interference of the NPs with the assay system. To each well, $44 \mu 1$ of ATP buffer, $2 \mu 1$ of ATP probe, 2 $\mu 1$ of ATP converter, and $2 \mu 1$ of developer solution were added. The plate was incubated for $30 \mathrm{~min}$ at RT and absorbance was measured at $570 \mathrm{~nm}$ in a microplate reader.

\subsubsection{PGC-1 $\alpha$ level detection}

PGC-1 $\alpha$ level was measured using PGC-1 $\alpha$ ELISA assay kit from BioSource (San Diego, CA). In brief, cells were lysed with two freeze-thaw cycles in ice-cold PBS and centrifuged at $5,000 \mathrm{~g}$ for $5 \mathrm{~min}$ at $4^{\circ} \mathrm{C}$. Supernatant was collected and assayed immediately. Each of the samples or standards $(100 \mu \mathrm{l})$ was added to the assigned well in the provided 96-well plate and incubated for $2 \mathrm{~h}$ at $37^{\circ} \mathrm{C}$. CdTe-QDs alone were added to separate wells to test for the interference of the NPs with the assay system. Biotinantibody $(100 \mu \mathrm{l})$ was then added to each well and the plate was incubated for $1 \mathrm{~h}$ at 
$37^{\circ} \mathrm{C}$. After three washes, $100 \mu \mathrm{l}$ of avidin-HRP was added to each well. After $1 \mathrm{~h}$ incubation and washes, $90 \mu 1$ of 3, 3', 5, 5' - tetramethylbenzidine (TMB) was added to each well followed by $15 \mathrm{~min}$ incubation. Stop solution $(50 \mu 1)$ was then added to each well and the absorbance was measured at $450 \mathrm{~nm}$ in a microplate reader.

\subsubsection{Statistical analysis}

Results were compared by one-way analysis of variance (ANOVA) followed by Dunnett's test for comparison of treatment groups to the negative control group and Tukey's test for pairwise comparisons among treatment groups. All data were expressed as mean \pm standard deviation. A value of $\mathrm{p}<0.05$ was considered as statistically significant.

\subsection{Results}

\subsubsection{Characterization of CdTe-QDs}

Spectral and size properties of CdTe-QDs that were used in this study have been performed and described in Chapter 2 of this thesis.

ICP-OES analysis of CdTe-QDs showed the levels of arsenic, calcium, chromium copper, iron, mercury magnesium manganese, and lead in the NP solution were below the detection limit (Table 3.1.). The concentration of cadmium and tellurium in the sample were $0.246 \mathrm{mg} / \mathrm{ml}(246 \mathrm{ppm})$ and $0.081 \mathrm{mg} / \mathrm{ml}(81 \mathrm{ppm})$, respectively (Table 3.1.). Since the concentration of CdTe-QDs that was used for the ICP-OES analysis was $2.5 \mathrm{mg} / \mathrm{ml}$, the ICP-OES result showed that these NPs contained $9.84 \%$ of cadmium and $3.24 \%$ of tellurium content. The cadmium content in CdTe-QDs from the ICP-MS analysis was 
approximate to the $10 \%$ content of cadmium in CdTe-QDs that was claimed by the manufacturer.

Table 3.1. ICP-OES analysis for determination of elements in CdTe-QDs. Elements of interest were confirmed using a minimum of two analytical wavelengths for each element. Five replicate readings were performed on each analyte.

\begin{tabular}{|c|c|}
\hline Element & Concentration (ppm) \\
\hline Arsenic & $<2.1$ \\
\hline Calcium & $2.6 \pm 0.0$ \\
\hline Chromium & $<0.2$ \\
\hline Copper & $<0.5$ \\
\hline Iron & $<0.1$ \\
\hline Mercury & $<4.1$ \\
\hline Magnesium & $<0.7$ \\
\hline Manganese & $<0.2$ \\
\hline Lead & $<0.6$ \\
\hline Cadmium & $246 \pm 5$ \\
\hline Tellurium & $81 \pm 20$ \\
\hline
\end{tabular}




\subsubsection{Cytotoxicity}

Cytotoxicity of CdTe-QDs, $\mathrm{CdCl}_{2}$ and $\mathrm{Na}_{2} \mathrm{TeO}_{3}$ in $\mathrm{HepG} 2$ cells was examined for changes in bioreduction activity using the MTT assay to estimate cellular capacity to reduce MTT to its formazan. Both $\mathrm{CdTe}-\mathrm{QDs}$ and $\mathrm{CdCl}_{2}$ caused cytotoxicity in HepG2 cells in a time- and dose-dependent manner (Figure 3.1.A) while $\mathrm{Na}_{2} \mathrm{TeO}_{3}$ treatment resulted in no significant cytotoxic effects (Figure 3.1.B). At the same concentration of cadmium, CdTe-QDs induced greater cytotoxic effects, compared to $\mathrm{CdCl}_{2}$. For CdTeQDs, the earliest changes were observed at $6 \mathrm{~h}$ with $0.1 \mu \mathrm{g} / \mathrm{ml}$ cadmium, and the lowest observable effects were observed with $0.01 \mu \mathrm{g} / \mathrm{ml}$ at $12 \mathrm{~h}$ exposure. At the longest exposure duration (24 h), CdTe-QDs caused maximal effects being $\sim 60 \%$ relative to control (Figure 3.1.). Examination by microscopy showed that, even at this high dose and exposure, cells had not detached (data not shown) and most still retained at least some capacity to reduce MTT to formazan, albeit at a much lower level compared to PBS-treated controls.

\subsubsection{Mitochondrial morphology}

The effects of CdTe-QDs on HepG2 morphology were studied using TEM. TEM results revealed changes in mitochondrial structure and shape including mitochondrial swelling and loss of cristae (Figures 3.2.B and D) compared to mitochondria from control cells (Figures 3.2.A and C). However, TEM results did not show the localization of the nanoparticles inside treated cells or their mitochondria. 
$\mathbf{A}$

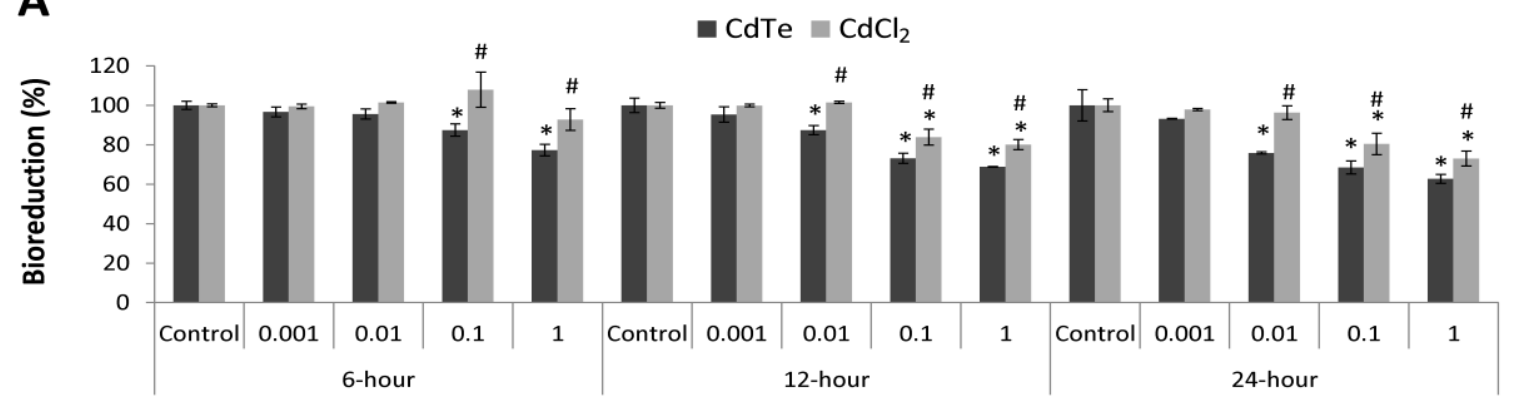

Concentration expressed in cadmium content $(\mu \mathrm{g} / \mathrm{ml})$

B

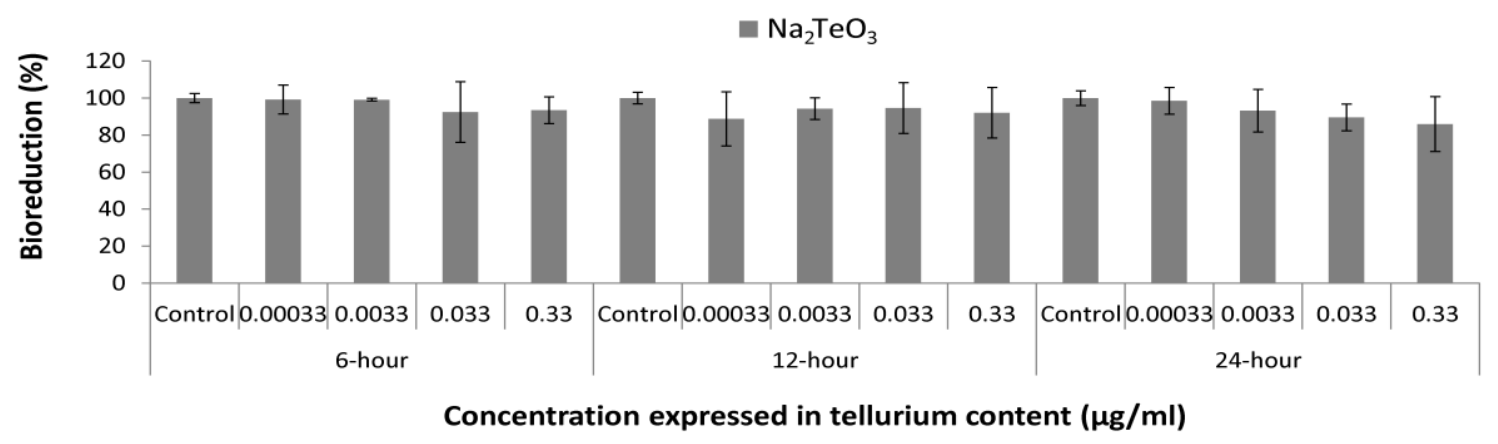

Figure 3.1. Cytotoxicity in HepG2 cells as assessed by loss of MTT bioreduction activity of CdTe-QDs, $\mathrm{CdCl}_{2}$, and $\mathrm{Na}_{2} \mathrm{TeO}_{3}$ treated cultures relative to PBS treated controls. (A) Cells were treated with different concentrations of CdTe-QD and $\mathrm{CdCl}_{2}$, at equivalent concentrations of cadmium, for 6,12 , and $24 \mathrm{~h}$. The concentrations of CdTeQDs and $\mathrm{CdCl}_{2}$ were expressed in cadmium concentrations. (B) Cells were treated with different concentrations of $\mathrm{Na}_{2} \mathrm{TeO}_{3}$ for 6,12 , and $24 \mathrm{~h}$. The concentrations of $\mathrm{Na}_{2} \mathrm{TeO}_{3}$ were expressed in tellurium concentrations that corresponded to the concentrations of cadmium in CdTe-QDs used in (A). Data points represent the means of three independent experiments done in duplicate \pm standard deviations. The asterisks $\left(^{*}\right)$ indicate statistically significant differences compared to PBS-treated control $(p<0.05)$. The number (\#) sign indicates statistically significant differences of $\mathrm{CdCl}_{2}$ compared to the CdTe-QD-treated group $(\mathrm{p}<0.05)$. 
A

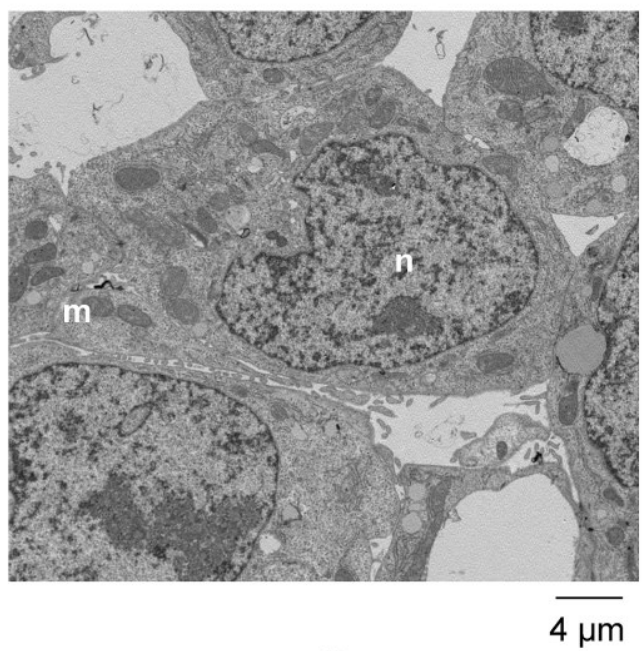

C

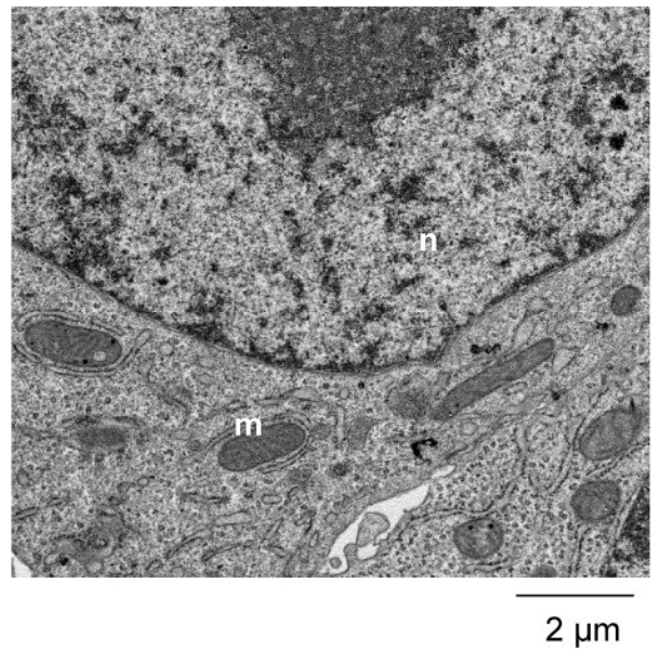

B

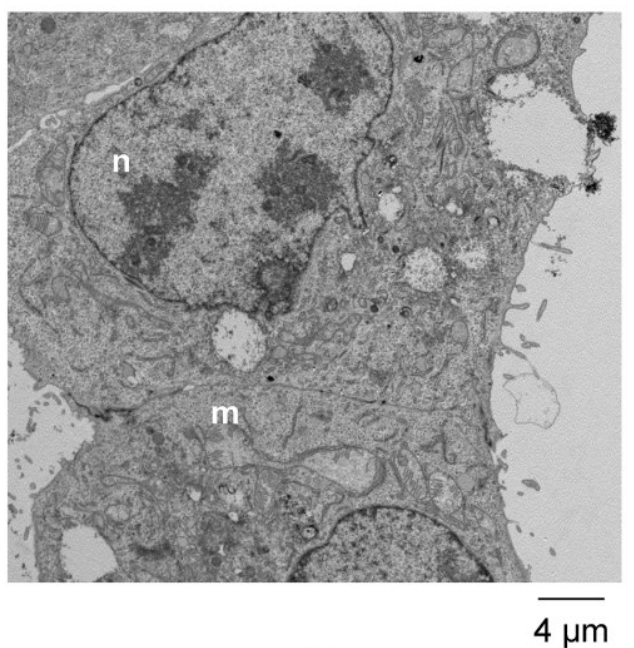

D

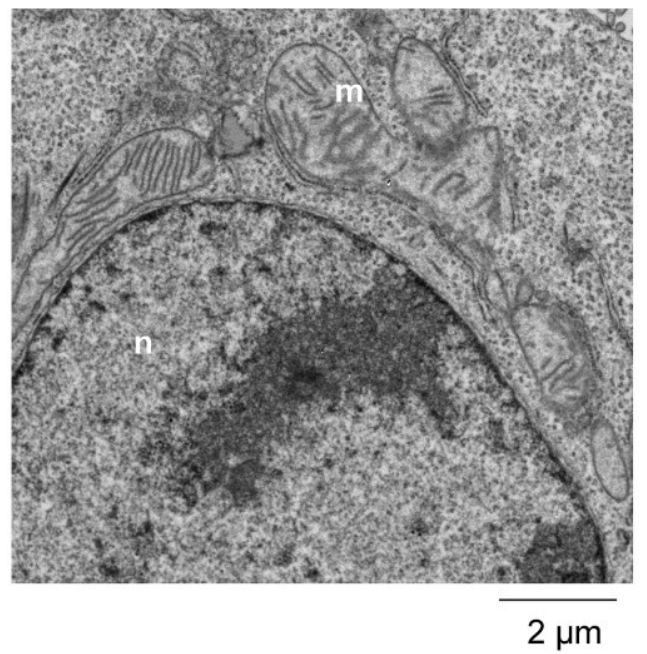

Figure 3.2. Transmission electron micrographs of HepG2 cells showing the effects of CdTe-QDs on mitochondrial morphology and structure. Cells were treated with $10 \mu \mathrm{g} / \mathrm{ml}$ CdTe-QDs (containing $1 \mu \mathrm{g} / \mathrm{ml}$ of cadmium) for $24 \mathrm{~h}$. (A) and (C) PBS-treated control at low and high magnifications. (B) and (D) CdTe-QD treated cells at low and high magnifications. (n) nuclear, (m) mitochondrion. 


\subsubsection{Detection of CdTe-QDs in isolated mitochondria}

To examine the association of CdTe-QDs with mitochondria, mitochondria from CdTe-QD treated and control cultures were isolated and measured by fluorimetry at 540 $\mathrm{nm}$ (emission wavelength for green CdTe-QDs). The results showed that mitochondria from the CdTe-treated cultures gave 2.5-to 3-fold $(\mathrm{p}<0.05)$ higher fluorescence intensity (FI) at $540 \mathrm{~nm}$ compared with the controls, demonstrating that CdTe-QDs were associating at relatively high levels with these organelles (Figure 3.3.).

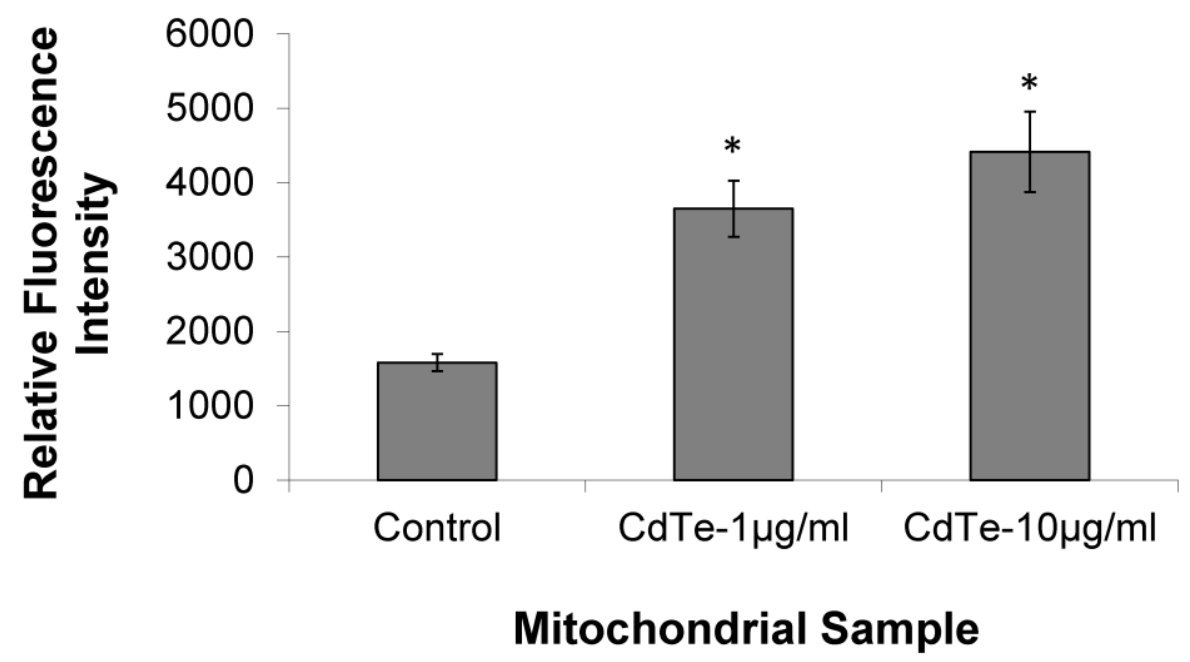

Figure 3.3. Fluorometric detection of CdTe-QDs in enriched mitochondrial fractions. Fluorescence intensity was measured at an excitation of $485 \mathrm{~nm}$ and emission of $540 \mathrm{~nm}$. Each data point represents the mean \pm standard deviation. The asterisks $(*)$ indicate statistically significant differences compared with the PBS-treated control $(\mathrm{p}<0.05)$.

\subsubsection{Mitochondrial membrane potential}


Loss or disruption of mitochondrial membrane potential was investigated by staining CdTe-QD-or $\mathrm{CdCl}_{2}$-treated and control cells with TMRE. The TMRE staining showed that both CdTe-QDs and $\mathrm{CdCl}_{2}$ induced a decrease in overall fluorescence, compared with the control (Figures 3.4.A, B and C), indicating a disruption of the membrane potential. In this assay, CCCP, a depolarizing agent, was used as the positive control and also resulted in a decrease in TMRE fluorescence (Figure 3.4.D).
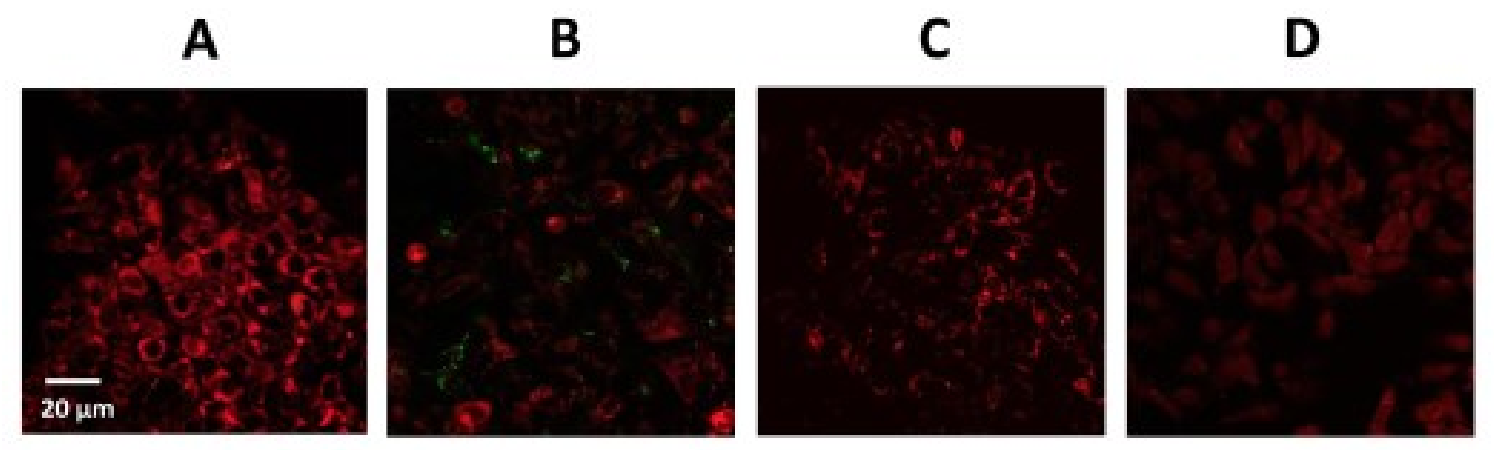

Figure 3.4. Confocal micrographs of HepG2 cells showing the effects on mitochondrial membrane potential induced by CdTe-QDs and $\mathrm{CdCl}_{2}$, at an equivalent concentration of cadmium (1 $\mu \mathrm{g} / \mathrm{ml})$, using TMRE staining. (A) PBS-treated control, (B) CdTe-QDs, which appeared as green dots/aggregates on the micrograph, (C) $\mathrm{CdCl}_{2}$, and (D) $\mathrm{CCCP}$, as a positive control.

\subsubsection{Intracellular calcium level}

The level of intracellular calcium was measured from confocal micrographs using a calcium indicator dye. The staining results showed that treatment with CdTe-QDs led to a significant increase in fluorescence intensity compared to the control ( 7.4 fold, $p<0.05$ ) 
(Figures 3.5.B and D). The results revealed that CdTe-QDs induced elevated intracellular $\mathrm{Ca}^{2+}$ levels. Treatment of $\mathrm{CdCl}_{2}$ also resulted in increase in calcium levels; however, at the same concentration of cadmium, the effect of $\mathrm{CdCl}_{2}$ was much less compared to that of CdTe-QDs (Figures 3.5.C and D).
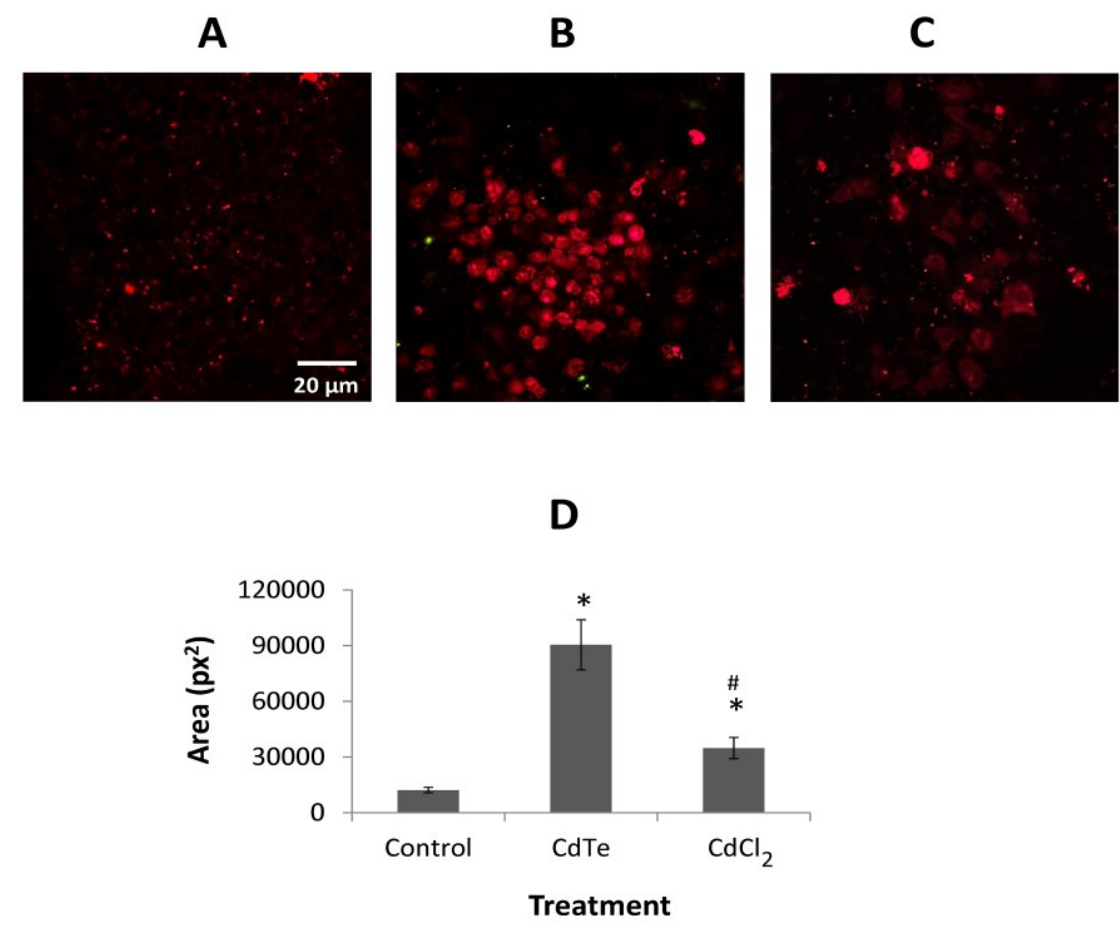

Figure 3.5. Confocal micrographs showing intracellular $\mathrm{Ca}^{2+}$ levels in HepG2 cells. (A) PBS-treated control, (B) cells treated with $10 \mu \mathrm{g} / \mathrm{ml}$ CdTe-QDs (containing $1 \mu \mathrm{g} / \mathrm{ml}$ of cadmium) for $24 \mathrm{~h}$, (C) cells treated with $1.63 \mu \mathrm{g} / \mathrm{ml} \mathrm{CdCl}_{2}$ (containing $1 \mu \mathrm{g} / \mathrm{ml}$ of cadmium) for $24 \mathrm{~h}$. Intracellular $\mathrm{Ca}^{2+}$ is indicated with red fluorescence. (D) The total area of red fluorescence (pixels squared: $\mathrm{px}^{2}$ ) was analysed with image analysis software using three micrographs for each sample. Each data point represents the mean \pm standard deviation. The asterisks $(*)$ indicate statistically significant differences compared with the PBS-treated control $(\mathrm{p}<0.05)$. The number $(\#)$ sign indicates statistically significant difference compared to the CdTe-QD-treated group $(\mathrm{p}<0.05)$. 


\subsubsection{Cellular respiration}

Cellular respiration is usually measured by assessing the rate of oxygen consumption. In this study, cellular respiration was measured using a phosphorescent oxygen sensitive probe to monitor the oxygen consumption in isolated mitochondria. Respiratory substrates, ADP, and inhibitors for respiratory chain complexes were used to study the effect of CdTe-QDs on different states of cellular respiration. To study the effect on state 2 (or basal respiration), either glutamate/malate (substrate for Complex I) or succinate (substrate for Complexes II and III) was added to a mitochondrial sample with or without CdTe-QDs. The oxygen consumption was measured in a microplate reader. The depletion of oxygen in the sample is reflected as an increase in fluorescence intensity. For state 3 respiration, exogenous ADP was added together with each substrate.

The results showed that CdTe-QDs induced significant decreases in the rates of oxygen, compared with the control (Figures 3.6.A and B), indicating the inhibition in oxygen consumption in the mitochondrial respiratory chain induced by the NPs. The CdTe-QD inhibitory effect was greater for respiration with succinate than for that with glutamate/malate as the substrate. For both substrates, the inhibition appeared greater for state 3 than for state 2 (Figures 3.6.A and B). In this assay, the inhibitors of mitochondrial complexes such as rotenone (Complex I) and malonate (Complex II) were included as the positive controls, which also showed inhibition of oxygen consumption. 
A

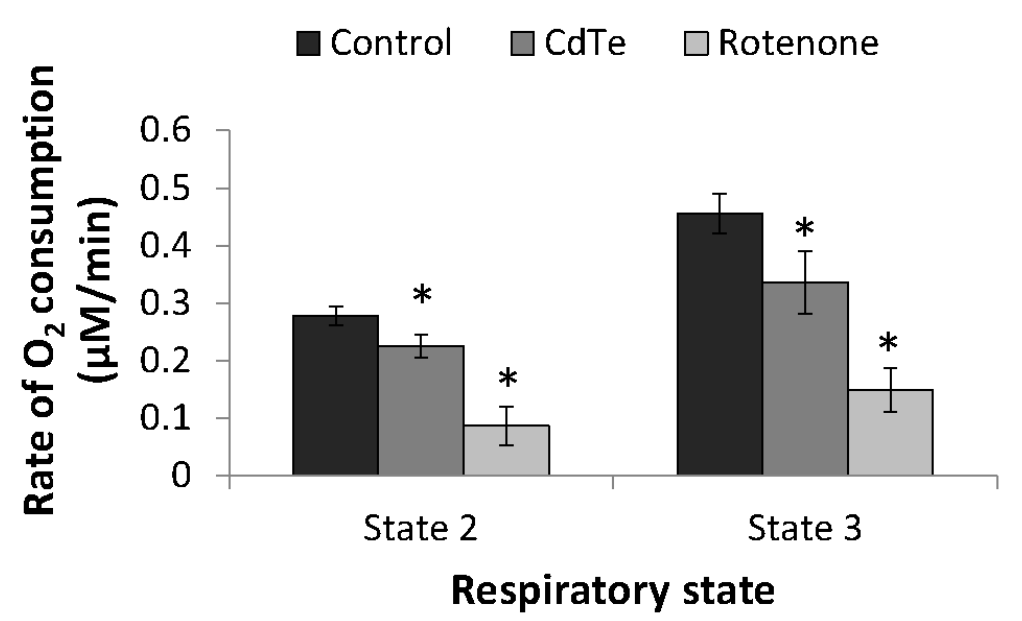

B

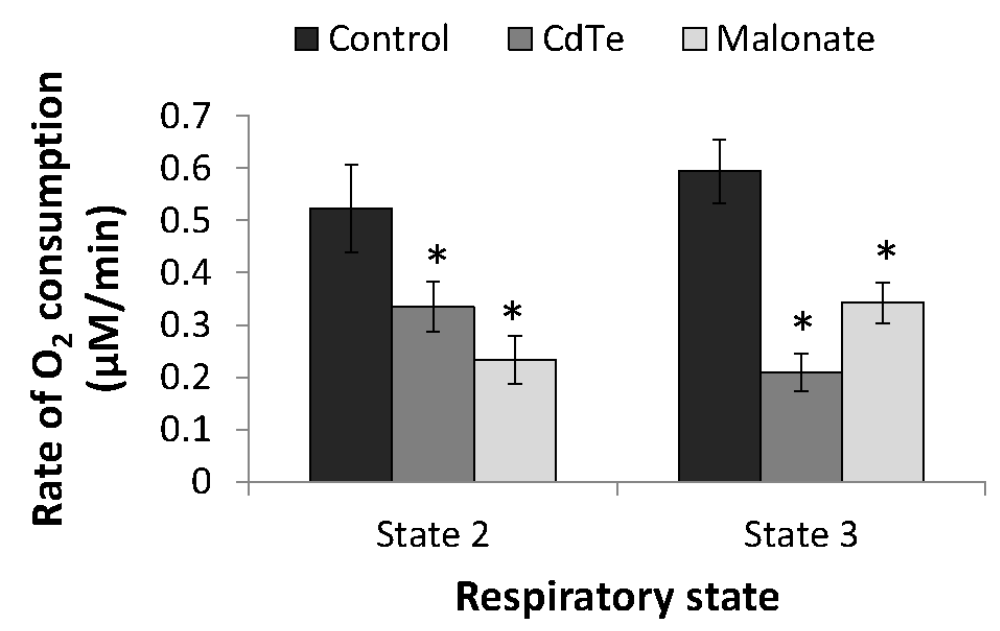

Figure 3.6. Effects of CdTe-QDs on cellular respiration expressed in rates of $\mathrm{O}_{2}$ consumption from isolated mitochondria. (A) State 2 and state 3 respiration performed with Complex I substrate, glutamate/malate. Rotenone, a Complex I inhibitor, was used as the positive control. (B) State 2 and state 3 respiration performed with Complexes II substrate, succinate. Malonate, a Complex II inhibitor, was used as the positive control. Data points represent the means of three independent experiments \pm standard deviations. The asterisks $(*)$ indicate statistically significant differences compared to control $(\mathrm{p}<0.05)$. 


\subsubsection{ATP content}

ATP content was determined to assess the effects of CdTe-QDs on ATP synthesis in test cells. CdTe-QDs (10 $\mu \mathrm{g} / \mathrm{ml}$, containing $1 \mu \mathrm{g} / \mathrm{ml}$ of cadmium) caused a significant decrease $(2.28$ fold, $\mathrm{p}<0.05)$ in ATP concentration compared to the control. Treatment of $\mathrm{CdCl}_{2}$ also induced a significant reduction of ATP level, but the effect was less severe compared to that of CdTe-QDs (Figure 3.7.). For this assay, the possible interference of CdTe-QDs with the assay system was tested by having CdTe-QDs alone in the wells with assay reagents. These wells showed absorbance values similar to the background (data not shown), indicating that CdTe-QDs do not interfere with the assay results.

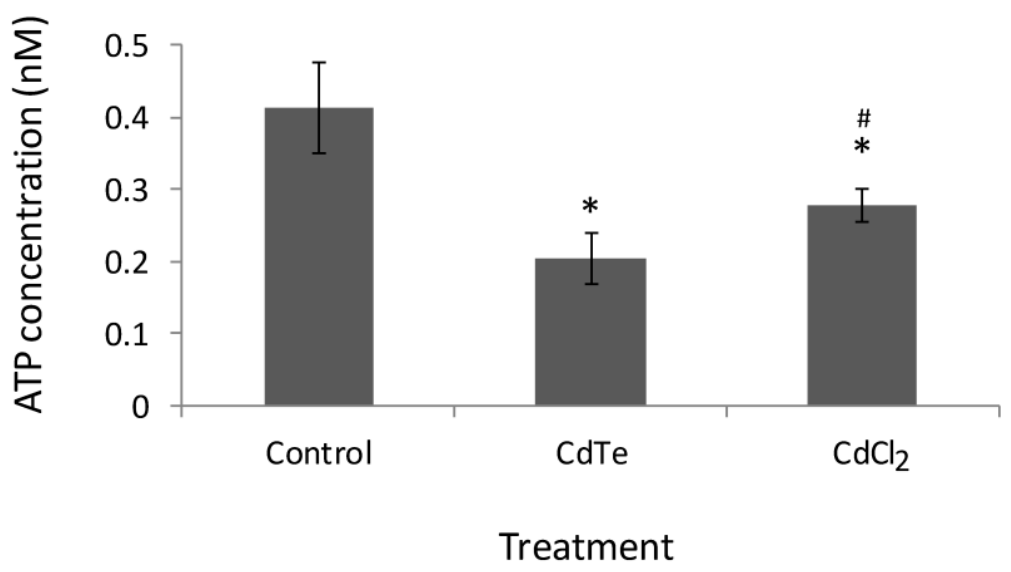

Figure 3.7. Effects of CdTe-QDs on ATP content in HepG2 cells. Cells were treated with CdTe-QDs and $\mathrm{CdCl}_{2}$, at an equivalent concentration of cadmium $(1 \mu \mathrm{g} / \mathrm{ml})$, for 24 h. Data points represent the means of three independent experiments \pm standard deviations. The asterisks $(*)$ indicate statistically significant differences compared to control $(\mathrm{p}<0.05)$. The number $(\#)$ sign indicates statistically significant difference compared to the CdTe-QD-treated group $(\mathrm{p}<0.05)$. 


\subsubsection{Electron transport chain component enzyme level and activity}

The quantity of mitochondrial complexes was determined using beadplex array assays. HepG2 cells were treated with CdTe-QDs or $\mathrm{CdCl}_{2}(1 \mu \mathrm{g} / \mathrm{ml}$ of cadmium $)$ for 24 h. The results showed that CdTe-QDs induced significant decreases $(p<0.05)$ in the levels of Complexes II, III, and IV, compared with the controls and an increase in the level of Complex $\mathrm{V}$ (Figure 3.8.A). $\mathrm{CdCl}_{2}$ treatment resulted in similar effects on the complex levels (Figure 3.8.A).

The effects of CdTe-QDs on mitochondrial complexes were further examined for changes in the activities of specific complex components. The results showed that CdTeQDs resulted in little or no change in Complexes I and V activities, but caused significant decreases $(\mathrm{p}<0.05)$ in Complexes II and, III, and IV activities (Figure 3.8.B). Similar effects on the activities of the complexes were observed with $\mathrm{CdCl}_{2}$ (Figure 3.8.B).

\subsubsection{PGC-1 $\alpha$ level}

The effect of CdTe-QDs on mitochondrial biogenesis was investigated by examining the change in PGC-1 $\alpha$, which is a transcriptional coactivator that regulates mitochondrial biogenesis. The level of PGC-1 $\alpha$ was measured using ELISA. Our result showed that treatment of CdTe-QDs $(10 \mu \mathrm{g} / \mathrm{ml}$, containing $1 \mu \mathrm{g} / \mathrm{ml}$ of cadmium) induced a significant increase $(18.2$ fold, $\mathrm{p}<0.05)$ in PGC-1 $\alpha$ levels in HepG2 cells at $24 \mathrm{~h}$, compared with the control (Figure 3.9.). Treatment of $\mathrm{CdCl}_{2}$, at the same cadmium concentration, also resulted in significant increase in PGC-1 $\alpha$ level, but the fold change induced by $\mathrm{CdCl}_{2}$ was less compared to that of CdTe-QDs (Figure 3.9.). The interference of CdTe-QDs with the assay system was tested in a similar manner to that of the ATP 
assay. CdTe-QDs showed no interference with the assay results as the wells that contained CdTe-QDs alone with the assay reagents showed the absorbance values similar to the background (data not shown).

A

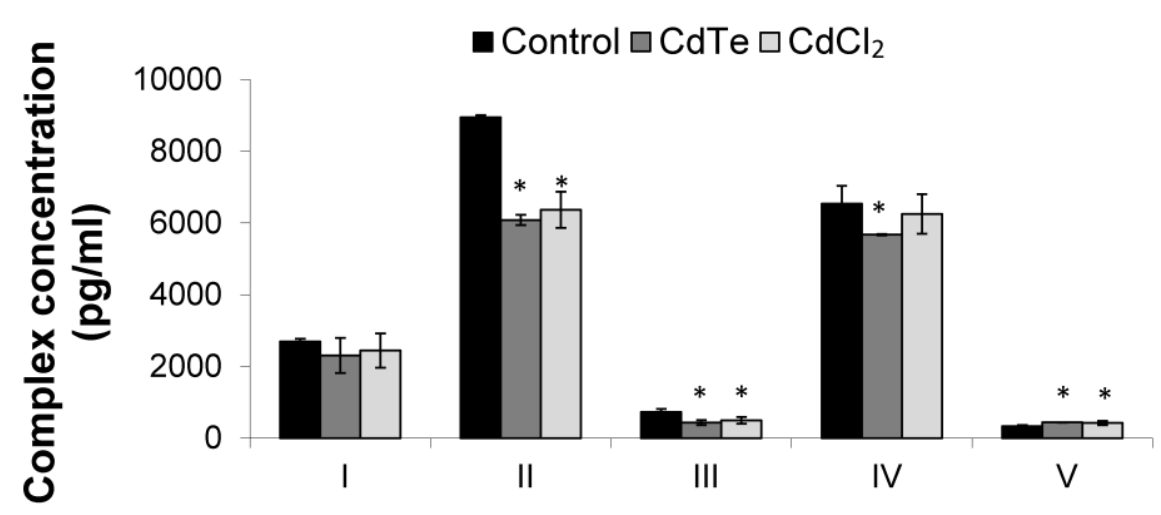

Mitochondrial Complex

B

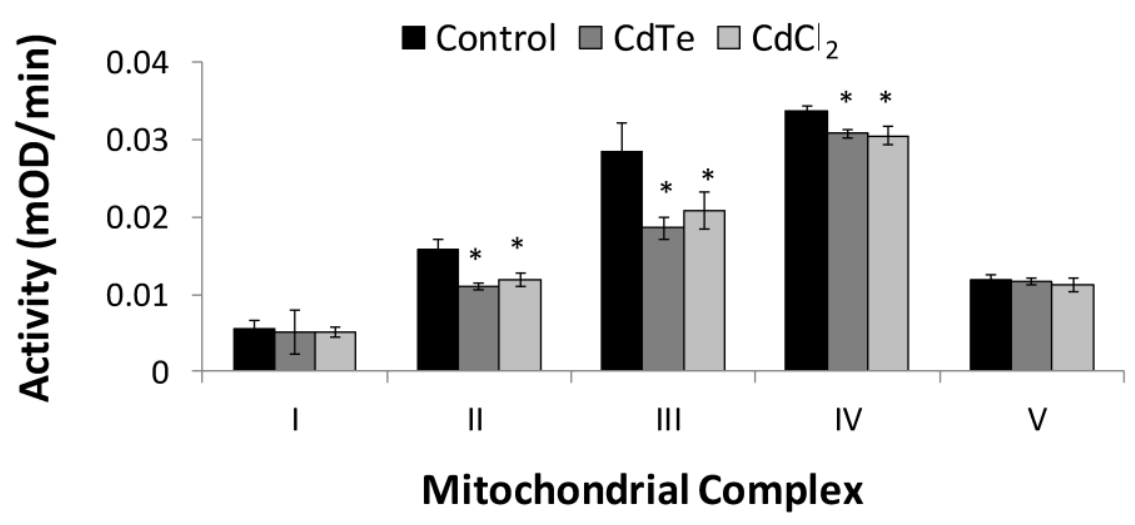

Figure 3.8. Effects of CdTe-QDs on mitochondrial ETC complexes. (A) Changes in ETC complex concentrations. (B) Changes in ETC complex activities. Cells were treated with CdTe-QDs and $\mathrm{CdCl}_{2}$, at an equivalent concentration of cadmium $(1 \mu \mathrm{g} / \mathrm{ml})$, for $24 \mathrm{~h}$. Data points represent the means of three independent experiments \pm standard deviations. The asterisks $(*)$ indicate statistically significant differences compared to control $(\mathrm{p}<0.05)$ 


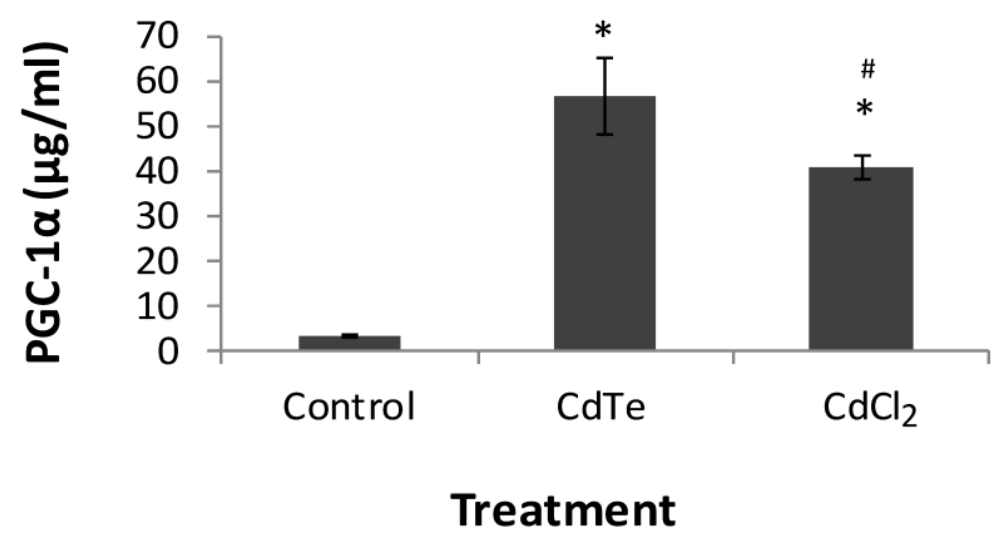

Figure 3.9. Effects of CdTe-QDs on mitochondrial biogenesis expressed as changes in PGC-1 $\alpha$ levels. Cells were treated with CdTe-QDs and $\mathrm{CdCl}_{2}$, at an equivalent concentration of cadmium $(1 \mu \mathrm{g} / \mathrm{ml})$, for $24 \mathrm{~h}$. Data points represent the means of three independent experiments \pm standard deviations. The asterisks $(*)$ indicate statistically significant differences compared to control $(\mathrm{p}<0.05)$. The number $(\#)$ sign indicates statistically significant difference compared to the CdTe-QD-treated group $(\mathrm{p}<0.05)$.

\subsection{Discussion}

Potential health effects of nanomaterials have been studied intensively in the past few decades. However, the detailed mechanisms of NP toxicity are poorly understood. Cadmium-based quantum dot toxicity has been reported in in vitro and in vivo studies (as reviewed by Hardman, 2006). In addition, our previous studies have shown that CdTeQDs caused suppression in immune responses to bacteria (Nguyen et al., 2013a), oxidative stress, and apoptosis via both extrinsic and intrinsic (mitochondrial) pathways (Nguyen et al., 2013b). It is important to investigate further the underlying mechanisms by which these NPs interact with, and affect the functions of cellular components. In particular, mitochondria have been suggested as possible targets for NPs. The aim of this 
study was to investigate the effects of CdTe-QDs on mitochondrial structure and function. Toward this goal, several hypothesis-driven experiments were developed for screening the mitochondrial toxicity caused by CdTe-QDs and also for investigating the mechanisms underlying the impacts on these organelles following QD exposures.

In initial experiments, cytotoxicity in HepG2 induced by CdTe-QDs was examined. The assay included $\mathrm{CdCl}_{2}$ at equivalent concentrations of cadmium for comparative purposes. CdTe-QDs induced cytotoxicity in HepG2 cell in a dose- and time-dependent manner. This result confirmed our previous studies (Nguyen et al., 2013a; Nguyen et al., 2013b) and aligned with the findings from other groups (Su et al., 2009; Zhang et al., 2007; Lovric et al., 2005b). $\mathrm{CdCl}_{2}$, at equivalent cadmium concentrations, also induced cytotoxicity in the same manner, but appeared to be less cytotoxic compared to CdTe-QDs. This suggests that the effects of CdTe-QDs cannot solely be explained by the action of the cadmium component of the NPs. Besides cadmium, the CdTe-QD core also contains tellurium which represents 3.24\% of the total NP mass, based on our ICP-OES analysis. To investigate the possible contribution of tellurium in CdTe-QD cytotoxicity, $\mathrm{Na}_{2} \mathrm{TeO}_{3}$ was used as a control. Tellurium concentrations were based on the mass ratio of tellurium/cadmium in the NPs. Our results showed that at tellurium concentrations appropriate for this study, no cytotoxicity was observed in HepG2 cells. Toxicity of tellurium has been previously reported in various cell lines including HeLa (Wen et al., 2013) and human chronic myeloid leukemia cells, K562 (Sandoval et al., 2012), and in immune cells isolated from different aquatic species (Bruneau et al., 2015). However, the tellurium doses that caused toxic effects in these cells were much higher than those we used in this present study. 
Therefore, our findings suggest that the effects of CdTe-QDs in this study were not from the tellurium component.

One of the effects of NPs is to cause changes in mitochondrial morphology and structure. Microscopy examination revealed that CdTe-QDs induced mitochondrial swelling and disruption of the normal appearance of their cristae. Our findings agree with a previous study by Cho and colleagues (2007), who reported that Cys-CdTe-QD treatments to MCF-7 cells resulted in significant mitochondrial swelling and rounding up as observed with confocal microscopy using a Mitotracker ${ }^{\circledR}$ dye. Our observations on the changes of mitochondrial morphology and structure with CdTe-QDs also support a recent study on $\mathrm{Cd} / \mathrm{Se} / \mathrm{Te}$-based QDs 705 toxicity in mice (Lin et al., 2013). This previous study also showed mitochondrial swelling, disorientation and reduction of mitochondrial number in the proximal convoluted kidney tubule cells of treated mice (Lin et al., 2013). Alteration of mitochondrial morphology and structure, induced by NPs, has also been observed with other types of NPs including DEP, ultrafine air pollution particles and multi-walled nanotubes. These changes were associated with the localization of NPs within mitochondria (Zhu et al., 2006; Oberdoster et al., 2005; Xia et al., 2006). In the current study, localization of CdTe-QDs within the cells or organelles was not observed clearly with TEM. However, the association of CdTe-QDs with mitochondria was inferred from an increase in fluorescence intensity of the QDs in isolated mitochondria of CdTe-QD treated cells. The association of CdTe-QDs with the mitochondrial enrichment suggests that mitochondria might be the targets of CdTe-QDs and supports the theory that NPs can be transported across cell membranes specifically into mitochondria (Foley et al., 2002). 
Alteration of mitochondrial morphology and structure is typically associated with damage of mitochondrial integrity including changes in mitochondrial membrane potential and membrane permeability (Petit et al., 1995). The mitochondrial membrane potential is a key indicator of cellular viability, as it reflects the pumping of hydrogen ions across the inner membrane during the process of electron transport and oxidative phosphorylation; the driving force behind ATP production (Chen, 1988). In this study, treatment with CdTe-QDs caused a disruption in mitochondrial membrane potential. Since a similar effect was observed with $\mathrm{CdCl}_{2}$, the disruption of mitochondrial membrane potential could also result from the effects of cadmium, which has also been previously reported by other groups (Martel et al., 1990; Bolduc et al., 2004; Mao et al., 2011; Liu et al., 2011). However, here we also observed the association of CdTe-QDs with mitochondria. Thus, the alteration of mitochondrial membrane potential observed in this study might also be resulted from the direct interaction of these NPs with the organelles causing physical damage and membrane potential collapse, which, in turn, results in mitochondrial swelling. Disruption of mitochondrial membrane potential has also been linked to apoptosis (Gottlieb et al., 2003). The collapse of the mitochondrial membrane potential coincides with the opening of the mitochondrial permeability transition pores, leading to the release of cytochrome $\mathrm{c}$ into the cytosol, which triggers other downstream events in the apoptotic cascade (Gottlieb et al., 2003). The disruption of mitochondrial membrane potential supports the findings from our previous studies showing that CdTe-QDs caused release of cytochrome c from mitochondria and apoptosis (Nguyen et al., 2013b). 
In the present study, treatment of Cd-Te-QDs resulted in an increase of intracellular $\mathrm{Ca}^{2+}$. A significant increase of cytosolic $\mathrm{Ca}^{2+}$ in murine J774A,1 macrophages from treatment with different surface-coated cadmium-based QDs was reported by Clift and colleagues (2010), who suggested the increases of $\mathrm{Ca}^{2+}$ was mediated via ROS and oxidative stress. Elevation of intracellular $\mathrm{Ca}^{2+}$ was also observed in $\mathrm{CdCl}_{2}$ treated cells, suggesting that the effect of CdTe-QDs on $\mathrm{Ca}^{2+}$ could also come from cadmium component of the NPs. Elevation of intracellular $\mathrm{Ca}^{2+}$ has been linked to apoptosis induced by cadmium (Yeh et al., 2009). It has been proposed that cadmium releases $\mathrm{Ca}^{2+}$ from endoplasmic reticulum and mitochondria in a phospholipase Cindependent manner (Yeh et al., 2009). However, in this current study, the effect from $\mathrm{CdCl}_{2}$ treatment was much less pronounced compared to that of CdTe-QDs at the same concentration of cadmium, suggesting that cadmium from the NPs is not the sole cause. The observed effect might also be caused by the direct interaction of CdTe-QDs with $\mathrm{Ca}^{2+}$ storing organelles within the cells. Calcium is the key regulator of mitochondrial function. Dysregulation of calcium homeostasis has been shown to play a critical role in mitochondrial dysfunction (Duchen, 2000). The opposite has also been shown in that mechanical injury of mitochondria can lead to disturbance in calcium homeostasis (Rodrigo and Standen, 2005). It is not clear whether the elevation of intracellular $\mathrm{Ca}^{2+}$ in this study is the cause or consequence of mitochondrial damage induced by NPs. It has been shown that elevated intracellular $\mathrm{Ca}^{2+}$ levels can lead to the excessive entry of calcium into the mitochondria, which triggers the opening of the mitochondria permeability transition pore (MPTP) and causes mitochondrial damage (Armstrong, 2006). Therefore, the elevated $\mathrm{Ca}^{2+}$ concentration might be the cause of the alterations of 
mitochondrial morphology, structure and membrane potential observed in this study. However, it is possible that the increase of intracellular $\mathrm{Ca}^{2+}$ in CdTe-QD treated cells is a result of mitochondrial injury caused by physical interaction between the nanoparticles and mitochondria and endoplasmic reticulum, where $\mathrm{Ca}^{2+}$ is stored within the cells, causing release of $\mathrm{Ca}^{2+}$ from these organelles (Chinopoulos and Adam-Vizi, 2006). Thus, the exact mechanism of elevation of intracellular $\mathrm{Ca}^{2+}$ induced by CdTe-QDs is not clear and remains to be further investigated.

Cellular respiration and ATP content are important indicators for mitochondrial function (Brand and Nicholls, 2011). Here, we demonstrated that CdTe-QDs reduced cellular respiration, expressed as oxygen consumption rate and depressed ATP synthesis. The majority of ATP production occurs via oxidative phosphorylation by the mitochondrial respiratory chain, which is essentially driven by the transmembrane electrical potential (Brown, 1992). Depression of ATP synthesis indicated effects of CdTe-QDs on the process of oxidative phosphorylation. Similarly, measurement of oxygen consumption has been considered the most informative parameter for indication of mitochondrial function since the measurement allows direct and specific assessment of the functioning of the ETC, the cornerstone of oxidative phosphorylation and cellular metabolism (Brown, 1992). Oxygen consumption by the mitochondria can be modulated by the uncoupling of oxidative phosphorylation, uncoupling proteins, the opening of the mitochondrial permeability transition pore, or the inhibition of individual components of the ETC (Brand and Nicholls, 2011). In the present study, the decrease in oxygen consumption induced by CdTe-QDs suggests the effects of these NPs on the ETC. Our findings agree with a recent study by Li and colleagues (2011) who also reported changes 
in cellular respiration in isolated rat liver mitochondria, induced by CdTe-QDs. These authors showed that CdTe-QDs significantly decreased respiratory rate at state 3 and suggested that the NPs affected both the ETC and transport through the mitochondrial inner membrane leading to uncoupled respiration inhibition (Li et al, 2011).

Our study went further to investigate the effects of CdTe-QDs on ETC component enzymes by examining their protein levels and activities. Treatment with CdTe-QD resulted in selective effects on ETC complexes. The NPs decreased the levels and activities of Complexes II, III and IV while causing no changes in the levels and activities of Complex I. Treatment of $\mathrm{CdCl}_{2}$ resulted in similar effects, suggesting that the effects of CdTe-QDs observed on ETC components were from cadmium toxicity. Several studies have reported inhibition of ETC components by cadmium (Belyaeva et al., 2012; Kurochokin et al., 2011; Wang et al., 2004). Cadmium has been shown to inhibit the activities of Complexes II (50-60\%) and III (30-77\%) of mitochondrial ETC from guinea pig liver, brain, and heart, but caused insignificant inhibition of Complexes I and IV activities (Wang et al., 2004). A study in isolated rainbow trout hepatic mitochondria showed equal inhibitory effects of cadmium on Complexes I, II, and III activities (Adiele et al., 2012). However, to date, there have been no studies on cadmium toxicity reporting the effects of this element on the levels of ETC enzymes, and the exact mechanism of how cadmium inhibits the mitochondrial ETC enzyme activities is unclear.

In this study, ETC enzyme concentrations were measured and there was a correlation between the effects of CdTe-QDs on the levels and the effects on the enzyme activities. Our study suggests that the decrease in ETC complex activities might be the result of the reduction in the levels of their proteins caused by CdTe-QDs. Similar to Complex I, the 
activity of Complex V was not affected by CdTe-QDs in this study; however, these NPs induced an increase in the level of this complex. This can be explained by the compensatory response of the cells to depressed ATP content. Our previous study has shown that CdTe-QDs induced ROS generation, leading to oxidative stress in the same cell line (Nguyen et al., 2013b). Mitochondria are the major source of ROS production in cells, and Complexes I and III are the primary sites of ROS production (Li et al, 2003). The impairment of electron transfer through Complexes I and III may induce ROS generation (Turrens et al., 1997). Here, the effects of CdTe-QDs on Complex III might be one of the sources of ROS production induced by the NPs that were observed in our previous work (Nguyen et al., 2013b).

Mitochondria biogenesis is defined as the growth and division of pre-existing mitochondria (Jornawaz and Shulman, 2010). PGC-1 $\alpha$ is a transcriptional coactivator, identified as a master regulator of mitochondrial number and function. The protein provides a direct link between external physiological stimuli and the regulation of mitochondrial biogenesis (Knutti and Kralli, 2001). Measurement of PGC-1 $\alpha$ levels was performed in this study to examine the effects of CdTe-QDs on mitochondrial biogenesis. The present study showed that CdTe-QDs caused a significant increase in PGC-1 $\alpha$ levels, suggesting that mitochondrial biogenesis may have been activated. Activation of PGC$1 \alpha$ has been reported as a result of cellular stress in which ROS were suggested to be one type of the signalling molecules to induce mitochondrial biogenesis (Acin-Perez et al., 2009). Overexpression of PGC-1 $\alpha$ was also linked to increases of cytosolic $\mathrm{Ca}^{2+}$ which led to the activation of calcium/calmodulin-dependent protein kinase II (CaMK II) and subsequently to the activation of p38 mitogen-activated protein kinase (MAPK) which is 
the upstream regulator of PGC-1 $\alpha$ (Wright et al., 2007). Elevation of PGC-1 $\alpha$ has also been shown as a response to ATP depletion and mitochondrial dysfunction in conditions such as hypoxia or ischemia (Rhonas et al., 2007; Garcia-Gimenez et al., 2011; St-Pierre, et al., 2006; Gutsaeva et al., 2008). Therefore, the increased PGC-1 $\alpha$ levels induced by CdTe-QDs observed in this study could be a result of oxidative stress, increased ROS production, and/or increased cytosolic $\mathrm{Ca}^{2+}$, as previously discussed. Overall, the elevation of PGC-1 $\alpha$ expression appeared to be a cellular compensatory response to the negative effects of CdTe-QDs on the mitochondrial function, including the decreases in cellular respiration and ATP synthesis induced by these NPs.

In conclusion, we conducted a hypothesis-driven investigation of mitochondrial toxicity induced by CdTe-QDs. Our study has revealed the mitochondria as the intracellular target of CdTe-QDs and demonstrated the effects of these NPs on mitochondrial morphology, structure and function in HepG2 cells. Mitochondrial swelling and disruption of mitochondrial membrane potential were evident along with elevation of intracellular $\mathrm{Ca}^{2+}$ levels, a decrease in cellular respiration and a depression of ATP synthesis. Effects on the levels and activities of ETC component enzymes appeared to be selective and explained the observed effects on mitochondrial critical functions and biogenesis. The role of cadmium in mitochondrial toxicity induced by CdTe-QDs was evident in this study; however, cadmium alone cannot explain the entire effects of these NPs. From our findings, it is possible that the NPs interact directly with mitochondria causing membrane damage and membrane potential disruption, as well as affecting ETC components which lead to inhibition of the oxidative phosphorylation process. 
Chapter 2 and 3 demonstrated in vitro toxicity of CdTe-QDs, identified the biological factors that contribute to toxicity, and revealed detailed underlying mechanisms that were related to mitochondria toxicity induced by these NPs. Although HepG2 cells are a suitale model for investigating hepatotoxicity and mitochondrial effects, validation of in vitro information from Chapter 2 and 3 with a mouse model is essential for understanding possible systemic and organ effects that might be relevant to health safety assessment. The murine exposure work was described in Chapters 4 and 5 of this thesis. 


\section{Chapter: Biodistribution and systemic effects of cadmium telluride quantum dot nanoparticles in a mouse model after intravenous administration}

This Chapter is adapted from the following manuscript:

Kathy C. Nguyen, Yan (Mary) Zhang, Julie Todd, Kelvin Kittle, Douglas Parks, Dominique Patry, Lorraine Casavant, Don Caldwell, Emmanuel Yumvihoze, Michelle Lalande, Martha Navarro, Andrey Massarsky, Alexandre Poulain, Thomas W. Moon, William G. Willmore and Azam F. Tayabali. 2015. Biodistribution and Toxic Effects of Cadmium Telluride Quantum Dot Nanoparticles in a Mouse Model. To be submitted to Nanomedicine journal.

For this manuscript, Kathy C. Nguyen designed and conducted these experiments and wrote the manuscript. Yan (Mary) Zhang assisted with animal necropsies. Julie Todd, Kelvin Kittle, Douglas Parks, Michelle Lalande, Dr. Martha Navarro assisted with animal exposure and necropsies. Dominique Patry performed hematology analysis. Lorraine Casavant, and Dr. Don Caldwell performed histology work. Emmanuel Yumvihoze and Dr. Alexandre Poulain assisted with ICP-Ms analysis. Dr. Andrey Massarsky performed the DLS analysis. Dr. Thomas Moon provided the instrument (Zetasizer Nano) for DLS measurement. Drs. Azam Tayabali and William Willmore provided input into the manuscript. Dr. Azam Tayabali and Health Canada provided funding for the project. 


\subsection{Abstract}

The objectives of this study are to investigate the tissue distribution and toxic effects of cadmium telluride quantum dot (CdTe-QD) nanoparticles in male BALB/c mice after intravenous injection. CdTe-QDs were observed in the blood, lung, heart, liver, spleen, kidney, testis and brain. The greatest accumulation of CdTe-QDs over time was in the liver. Accumulations in the spleen and kidney were also notably high. At highest doses, CdTe-QDs caused mild dehydration, lethargy, ruffled fur, hunched posture and body weight loss. Histological analysis of the tissues revealed hepatic hemorrhage and necrotic areas in the spleen. The sera of mice treated with high doses of CdTe-QDs showed significant increases in alanine aminotransferase, aspartase transaminase, and total bilirubin levels, as well as a reduction in albumin. CdTe-QDs reduced the number of platelets, and elevated total white blood cells including monocytes and neutrophils, serum amyloid A, and several pro-inflammatory cytokines. These results demonstrated that the liver is the main target of CdTe-QDs and that exposures to CdTe-QDs cause hepatic and splenic injury, as well as systemic effects, in mice. By contrast, cadmium chloride $\left(\mathrm{CdCl}_{2}\right)$, at an equivalent concentration of cadmium, appeared to have a different pharmacokinetic pattern from that of CdTe-QDs and induced negligible effects on the endpoints examined, suggesting that CdTe-QDs are more toxic to mice compared to cadmium alone.

\subsection{Introduction}

Nanomaterials (NMs) possess novel properties that lead to their widespread use in biotechnology applications and consumer products. The heightened production and use 
of NMs will result in greater exposure to consumers and workers. Therefore, the hazards associated with NMs need to be assessed. Quantum dots (QDs) are a class of engineered nanoparticles (NPs) that range from 2 to $100 \mathrm{~nm}$ in diameter (Bruchez et al., 1998). Owing to their unique optical and electronic properties, including broad absorption and narrow emission, QDs have been widely used in electronic and medical applications (De Wild et al., 2003).

Among the many types of QDs, those composed of cadmium such as CdSe and CdTe, have been mostly used in biological and medical research applications, thus these NPs have been most studied for their environmental and health risk. Although the toxicity of cadmium QDs has been demonstrated in various cell models and reported in a number of studies (as reviewed by Hardman, 2006; Pelley et al., 2009; Yong et al., 2013), information on the toxicity of these NPs in animal models is limited. To date, there have been only a few studies reporting toxicity of cadmium QDs in invertebrate and aquatic animal models (Gagne et al., 2008; Gagne et al., 2010; Kim et al., 2010; Lee et al., 2010; Pace et al., 2010; King-Heiden et al., 2009), or their effects in rodents (as reviewed by Yong et al., 2013; $\mathrm{Wu}$ and Tang, 2014). While the studies in mussels, rainbow trout, Daphna, and zebrafish have reported QD toxicity, such as immunotoxicity, oxidative stress, and genotoxicity, in these aquatic organisms, the reports of QD toxicity in rodents have been inconclusive. A few studies have shown toxicity of cadmium QDs in mice and rats including altered locomotor activity (Zhang et al., 2007), immunological effects (Tang et al., 2013), pulmonary vascular thrombosis leading to death (Geys et al., 2008; Tang et al., 2013), and injuries to liver, spleen, and kidneys (Liu et al., 2011; Tang et al., 2013). By contrast, several studies have reported no observable toxicity induced by 
cadmium QDs in rodents or in primates (Law et al., 2009; Hauck et al., 2010; Su et al., 2011; Haque et al., 2012; Ye et al., 2012). The discrepancy in mammalian toxicity of cadmium QDs may be explained by the variation in particle size, coating, dose, and exposure conditions that were used in the studies (Haque et al., 2013). Consequently, despite the effort by many research groups to characterize the behaviour and to measure the toxicity of QDs in vivo, little can be concluded about the biological effects of these NPs in relevant animal models or during human exposure.

The aims of this study are to investigate the biodistribution of CdTe-QDs and their toxic effects in BALB/c mice after intravenous injection. The presence of CdTeQDs was monitored in various tissues to determine its principle tissue targets. Several toxicological endpoints were employed to investigate tissue and systemic effects. $\mathrm{CdCl}_{2}$ was also used to compare the distribution and effects of cadmium to those of CdTe-QDs. The data provides important information on the behaviour of CdTe-QDs within an animal model and their potential health effects, which should be helpful to assess the human health risk of these NPs.

\subsection{Materials and Methods}

\subsubsection{Materials}

CdTe-QDs were purchased from Nano Impex Canada (Mississauga, ON). CdTeQDs were described by the manufacturer as mercaptopropionic acid (MPA) capped CdTe-QDs with a size of $5 \mathrm{~nm}$ and a spectral emission of $540 \mathrm{~nm}$. The stock of CdTeQDs was provided at a concentration of $5 \mathrm{mg} / \mathrm{ml}$ in water containing $20 \%(\mathrm{w} / \mathrm{w})$ of cadmium. Cadmium chloride $\left(\mathrm{CdCl}_{2}\right)$, hematoxylin, eosin, and Giemsa were obtained 
from Sigma-Aldrich (St. Louis, MO). Biochemical kits including alanine transaminase (ALT), aspartate aminotransferase (AST), alkaline phosphatase (ALP), albumin, total bilirubin, and creatinine were purchased from Fisher Scientific (Ottawa, ON). Serum amyloid A (SAA) kit was obtained from Life Technologies (Carlsbad, CA). Bio-Plex cytokine kits and reagents were purchased from Bio-Rad (Hercules, CA).

\subsubsection{Characterization of CdTe-QDs}

CdTe-QDs used in this study were characterized using transmission electron microscopy (TEM), dynamic light scattering (DLS), and spectrofluorometry. For TEM, CdTe-QD stocks were diluted by 1,000-fold with $\mathrm{dH}_{2} \mathrm{O}$, deposited on formvar-coated grids, and air-dried overnight at room temperature (RT). Grids were examined with a JOEL JEM 1230 operating at $60 \mathrm{kV}$. DLS analysis was performed on a Zetasizer Nano (Malvern Instruments Ltd). For this technique, CdTe-QD stocks were diluted 1,000-fold with liquid chromatography/mass spectrometry (LC/MS) grade water or physiological saline. One millilitre was added to a clean $12 \mathrm{~mm}$ polystyrene cuvette (DTS0012) and inserted into the instrument to obtain the readings. Each sample was measured at least 3 times. Spectral properties of CdTe-QDs were examined using spectrofluorometry. Fluorescence spectra of CdTe-QDs (340 nm excitation and emission wavelengths from $450 \mathrm{~nm}$ to $650 \mathrm{~nm})$ were obtained using $100 \mu \mathrm{l}(125 \mu \mathrm{g} / \mathrm{ml})$ of CdTe-QD solutions in a 96-well opaque plate and scanning with a SPECTRAmax GEMINI XS microplate spectrofluorometer (Molecular Devices, Sunnyvale, CA). A standard curve of fluorescence intensity (measured at $550 \mathrm{~nm}$ ) vs. concentration was made for each QD source using three replicates of serial dilutions ranging from $0 \mu \mathrm{g} / \mathrm{ml}$ to $250 \mu \mathrm{g} / \mathrm{ml}$. 


\subsubsection{Animal treatments and sample collection}

This study and all procedures involving animals were approved by the Health Canada Animal Care Committee. Male BALB/c mice ( 25 g) were purchased from Charles River Laboratories Inc. (Saint-Constant, Quebec). The mice were acclimated for one week prior to experimentation. Animals were housed in cages under a 12-hour (-h) light/dark cycle in a controlled temperature $\left(20 \pm 2^{\circ} \mathrm{C}\right)$ and relative humidity $(50 \pm 20 \%)$ environment and were given access to food and water ad libitum.

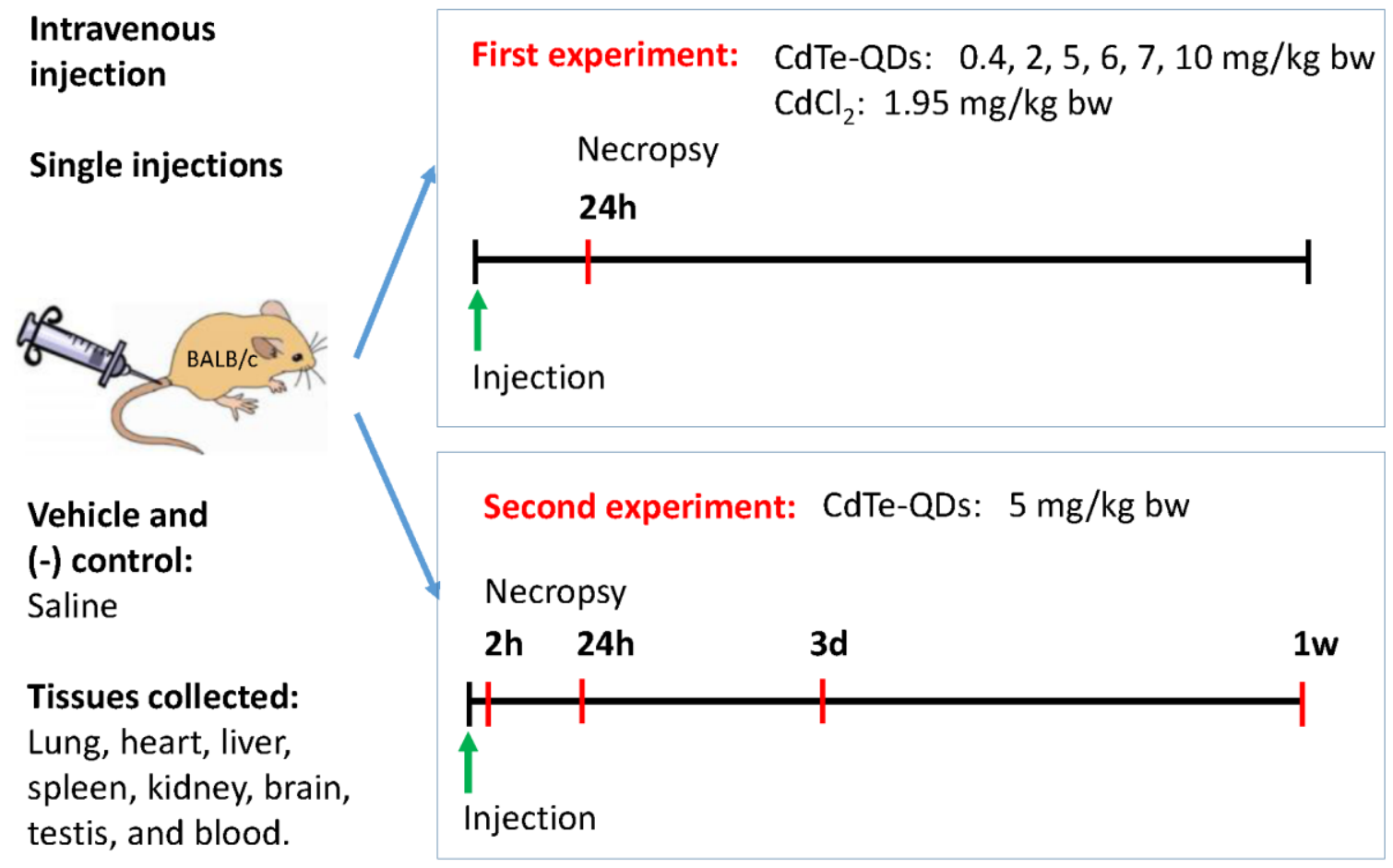

Figure 4.1. The animal exposure and necropsy scheme used in in vivo studies described in Chapter 4 and 5 of this thesis. 
Mice were exposed to CdTe-QDs or $\mathrm{CdCl}_{2}$ via intravenous injection into the tail vein. Physiological saline was used as the vehicle for the study. Working solutions of CdTe-QDs and $\mathrm{CdCl}_{2}$ were prepared by diluting the stock solution in saline. Eight mice were used for each control and treatment group. Mice were regularly monitored for physical activity and behaviour right after injection until necropsies. Body weights were taken right before the injection and before necropsies. Blood and tissues, including lung, heart, liver, spleen, kidney, brain and testis, were collected. The study was designed into two sets of experiments.

The first set of experiments was conducted to screen for the dose effects of CdTeQDs. A range of CdTe-QD concentrations $(0.4,2,5,6,7$, and $10 \mathrm{mg} / \mathrm{kg}$ body weight (bw)) was used to treat the mice, which were then examined $24 \mathrm{~h}$ after. From this first set of experiments, the threshold dose, the dose at which there was no observable changes in mouse behaviour and physical activities caused by CdTe-QD exposure, was determined and used for the second set of experiments. $\mathrm{CdCl}_{2}$ was also used in this first set of experiments to compare cadmium effects. A concentration of $1.95 \mathrm{mg} / \mathrm{kg}$ bw of $\mathrm{CdCl}_{2}$ was used to treat the mice. This concentration of $\mathrm{CdCl}_{2}$ contains $1.2 \mathrm{mg} / \mathrm{kg}$ bw $(61.3 \%)$ of cadmium, which is an equivalent concentration of cadmium in $6 \mathrm{mg} / \mathrm{kg}$ bw of CdTeQDs since these NPs contains $20 \%$ of cadmium.

In the second set experiments, the threshold dose from the first set of experiments, which was $5 \mathrm{mg} / \mathrm{kg}$ bw, was used to treat the mice. Different post-exposure sampling times, 2 h, 24 h, 3 days (d), and 1 week (w) were used in subsequent experiments to examine the progression of effects and the recovery of the mice. The detailed animal exposures and dissections were outlined in Figure 4.1. 


\subsubsection{Inductively coupled plasma mass spectrometry (ICP-MS)}

The detection and quantification of cadmium from CdTe-QDs and $\mathrm{CdCl}_{2}$ in the blood and tissues were carried out using ICP-MS. Multi-element standard and multiinternal standard were obtained from Agilent (Santa Clara, CA). Nitric acid for trace metal analysis was purchased from Sigma Aldrich (St. Louis, MO). Standards and reagents were prepared using Milli-Q (Millipore, Etobicoke, ON). Samples were digested following DigiPrep application note for tissue digestion adapted from USEPA methods 200.2 revision 2.8. Briefly, blood and tissue samples were digested with $2 \mathrm{ml}$ 10.3 $\mathrm{M} \mathrm{HNO}_{3}$ for $60 \mathrm{~min}$ at $95^{\circ} \mathrm{C}$, utilizing a digestion system (DigiPREP MS, SCP Science, France). Samples were topped up to a final volume of $5 \mathrm{ml}$ with MilliQ ${ }^{\circledR}$ water. For QA/QC, blanks and certified reference material were used. Samples were then analyzed using an Agilent 7700x ICP-MS.

\subsubsection{Spectrofluorometry and confocal microscopy}

The presence of CdTe-QDs in the blood and tissues was analysed using spectrofluorometry and confocal microscopy. For the presence of CdTe-QDs in the blood, blood samples from the control and treated mice were smeared on microscopic slides, dried, and analysed with a Nikon TE2000 microscope attached to a C1 confocal unit (Nikon Canada Inc. Mississauga ON). For the presence of CdTe-QDs in tissues, a set of tissues (100 mg of each tissue) were homogenized in $500 \mu$ l water and the homogenates were then diluted and measured for florescence intensity (FI) using CdTeQD excitation and emission wavelengths (340 nm and $550 \mathrm{~nm}$, respectively), in a SPECTRAmax GEMINI XS microplate spectrofluorometer (Molecular Devices, 
Sunnyvale, CA). Another set of tissues were sectioned and then analysed with a confocal microscope (Nikon Canada Inc. Mississauga ON).

\subsubsection{Pathology/Histology}

Small pieces (approximate $3 \mathrm{~mm}^{3}$ in size) of tissues were fixed with $10 \%$ formaldehyde, embedded into paraffin, sectioned into $5 \mu \mathrm{m}$ thick sections, and mounted on a glass microscope slide using standard histopathological techniques. The sections were stained with $\mathrm{H} \& \mathrm{E}$ and examined with a light microscope. Blood smears were stained with Wright's stain and examined with a light microscope.

\subsubsection{Biochemical tests}

Serum albumin, alanine aminotransferase, aspartase transaminase, total bilirubin albumin, and creatinine were measured using a Clinical Chemistry benchtop analyzer (Horiba Medical, Irvine, CA). Assays were conducted as described by the manufacturer.

\subsubsection{Haematology}

Following anaesthesia with isoflurane, $500-1000 \mu \mathrm{L}$ of blood was immediately collected by cardiac puncture and transferred to tubes containing ethylenediaminetetraacetic acid (EDTA). Blood was analyzed using an automated hematology analyzer (Sysmex XT-2100, Kobe, Japan), as described by the manufacturer.

\subsubsection{Serum amyloid A (SAA) assay}


SAA levels were determined using a mouse SAA ELISA kit (Life Technologies). The assays were performed according to the manufacturer's instructions. Briefly, serum samples were diluted 1:200 in the provided sample diluent buffer. To each well of a 96well plate pre-coated with SAA antibody, $50 \mu 1$ of anti SAA/HRP conjugate was added followed by $50 \mu \mathrm{l}$ of standard or sample. After a $1 \mathrm{~h}$ incubation, the plate was washed four times with washing buffer. After washing, $100 \mu 1$ of TMB substrate was added to each well and the plate was incubated for 15 min. Stop solution $(100 \mu l)$ was then added to each well and the plate was read at $450 \mathrm{~nm}$ in a microplate reader (Molecular Devices, Sunnyvale, CA).

\subsubsection{Serum cytokine measurement}

The serum cytokine/chemokine levels were measured using the Luminex-based Bio-Plex array system (Bio-Rad, Hercules, CA) according to the manufacturer's instructions. Briefly, anti-cytokine/chemokine conjugated beads were added to individual wells of a 96-well filter plate. Beads were then sedimented using vacuum filtration and washed briefly with kit wash buffer. Fifty microliters of pre-diluted standards or serum samples were added and incubated for 30 min at RT with gentle shaking. The filter plate wells were then washed again before adding $25 \mu 1 /$ well of prediluted detection antibody and incubating for $30 \mathrm{~min}$ at RT. After washing, $50 \mu 1 /$ well of pre-diluted streptavidin-conjugated phycoerythrin was added and the plate was shaken for

$10 \mathrm{~min}$. The wells were again washed and $125 \mu \mathrm{l} /$ well of assay buffer was added. The plate was shaken for 1 min and analyzed with the Bio-Plex 100 array system. 


\subsubsection{Statistical analysis}

Results were compared by one-way analysis of variance (ANOVA) followed by Dunnett's test for comparison of treatment groups to the negative control group and Tukey's test for pairwise comparisons among treatment groups. All data was expressed as mean \pm standard deviation. A value of $p<0.05$ was considered as statistically significant.

\subsection{Results}

\subsubsection{Characterization of CdTe-QDs}

Transmission electron microscopy (TEM) examination showed that the average size of CdTe-QDs, diluted in water, was $15.25 \pm 0.34 \mathrm{~nm}$ (Figure 4.1.A). TEM also revealed agglomeration of the NPs. Analysis with dynamic light scattering (DLS) of CdTe-QDs, dispersed in water and saline, showed the average sizes were $3.96 \pm 0.48$ and $3.47 \pm 0.49 \mathrm{~nm}$, respectively (Figure 4.1.B). The fluorescence spectra of CdTe-QDs in water and saline showed the maximum emission was at $550 \mathrm{~nm}$ (Figure 4.1.C). Standard curves were plotted using dilutions of known concentrations of CdTe-QDs. The standard curves of CdTe-QDs in water and in saline were relatively linear as indicated by the good curve fit $\left(\mathrm{R}^{2}=0.994\right.$ in water and $\mathrm{R}^{2}=0.992$ in saline) (Figure 4.1.D). The fluorescence intensity of CdTe-QDs in saline at optimal emission was greater than that of CdTe-QDs in water, suggesting the effects of $\mathrm{Na}^{+}$and $\mathrm{Cl}^{-}$ions on the spectral property of these NPs. 
A

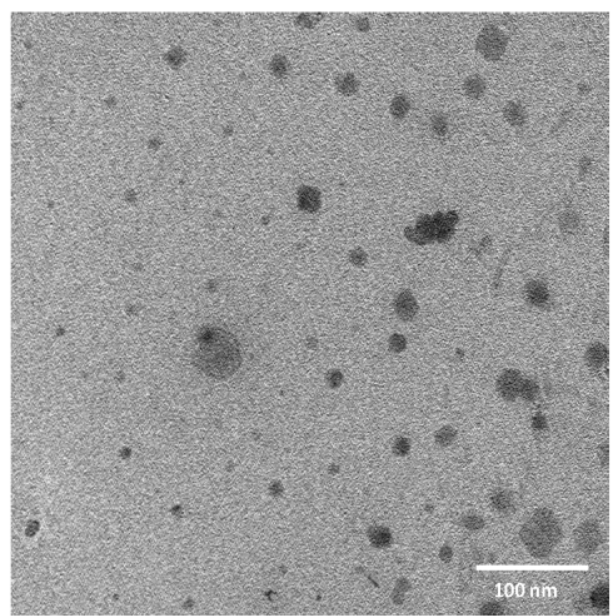

C

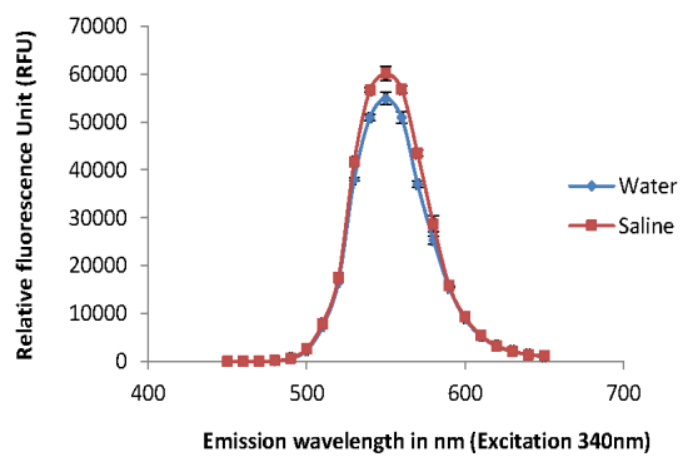

B

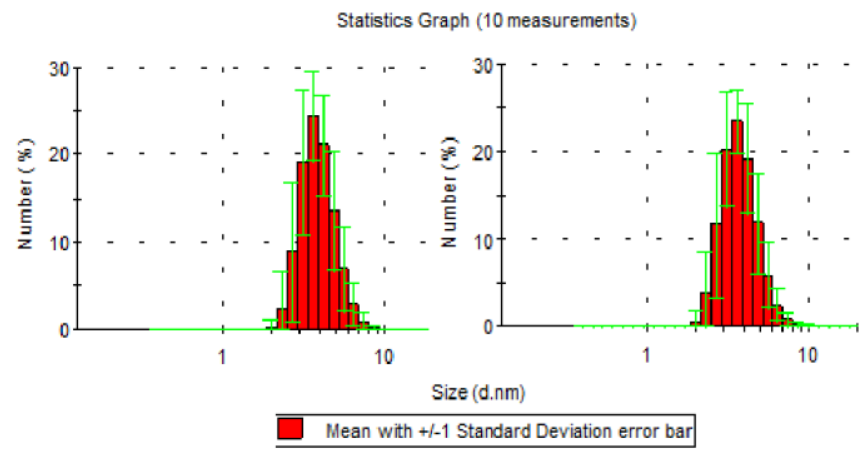

\begin{tabular}{|l|c|c|}
\hline Diluent & Water & Saline \\
\hline Average size & $3.9 \pm 0.4$ & $3.4 \pm 0.5$ \\
\hline
\end{tabular}

D

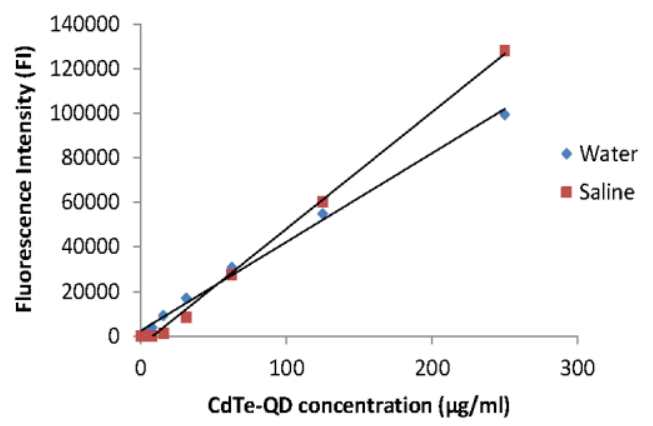

Figure 4.2. Characterization of CdTe-QDs in water and saline. (A) TEM analysis of diluted CdTe $(1: 1,000)$ deposited on formvar-coated copper grids. (B) DLS analysis of diluted CdTe-QDs (1:1,000). (C) Fluorescence spectra of CdTe-QDs at $125 \mu \mathrm{g} / \mathrm{ml}$ using excitation (Ex.) of $340 \mathrm{~nm}$. (D) Fluorescence standard curves of CdTe-QDs (from 0 $\mu \mathrm{g} / \mathrm{ml}$ to $250 \mu \mathrm{g} / \mathrm{ml}$ ) using excitation (Ex.) of $340 \mathrm{~nm}$ and emission (Em.) of $550 \mathrm{~nm}$. 


\subsubsection{Detection of cadmium in blood and tissues using ICP-MS}

Biodistribution of CdTe-QDs in the blood and tissues of BALB/c mice was examined using ICP-MS after intravenous administration. At $24 \mathrm{~h}$ with an administration dose of $5 \mathrm{mg} / \mathrm{kg}$ bw, ICP-MS results showed that CdTe-QDs distributed to all organs examined including the lung, heart, liver, spleen, kidney, brain and testis (Table 4.1.). At $2 \mathrm{~h}$, the level of cadmium was the greatest in the liver, but blood, spleen and kidney were also substantially higher than in other tissues. From $2 \mathrm{~h}$ to $1 \mathrm{w}$ of exposure, the level of cadmium was decreasing over time in the blood and heart while increasing in the liver, spleen, kidney and testis. The levels of cadmium in the liver, spleen and kidney reached the highest levels at $3 \mathrm{~d}$ and remained at similar levels at $1 \mathrm{w}$, while that in the lung reached the highest at $24 \mathrm{~h}$ post-exposure and remained approximately the same at $3 \mathrm{~d}$ and $1 \mathrm{w}$. Accumulation of CdTe-QDs appeared greatest in the liver over time of exposure (Table 4.1.).

A comparison of the distribution of CdTe-QDs and $\mathrm{CdCl}_{2}$ in tissue was made using $6 \mathrm{mg} / \mathrm{kg}$ bw of CdTe-QDs and $1.95 \mathrm{mg} / \mathrm{kg}$ bw of $\mathrm{CdCl}_{2}$, which contain equivalent cadmium concentrations. Cadmium was measured at $24 \mathrm{~h}$ post-exposure using ICP-MS (Table 4.2.). The cadmium level from $\mathrm{CdCl}_{2}$ was very low in the blood at $24 \mathrm{~h}$ compared to that of CdTe-QDs, which was substantial. Cadmium from $\mathrm{CdCl}_{2}$ appeared greatest in the liver and kidney, but was low in other tissues. Although an equivalent cadmium concentration was used for both $\mathrm{CdTe}-\mathrm{QDs}$ and $\mathrm{CdCl}_{2}$, the levels of cadmium from $\mathrm{CdCl}_{2}$ appeared significantly lower than those from CdTe-QDs in all tissues (Table 4.2.). 
Table 4.1. Cadmium concentrations ( $\mu \mathrm{g} / \mathrm{kg}$ tissue) in the blood and tissues as detected by ICP-MS. Mice were treated with $5 \mathrm{mg} / \mathrm{kg}$ bw of CdTe-QDs for different time points.

\begin{tabular}{|l|c|c|c|c|cc|}
\hline Sample & Control & 2h & 24h & 3d & 1w \\
\hline BLOOD & $0.0 \pm 0.0$ & $4028 \pm 127$ & $1863 \pm 287$ & $589 \pm 78$ & $109 \pm 25$ \\
\hline LUNG & $1.2 \pm 0.0$ & $845 \pm 67$ & $1143 \pm 98$ & $1126 \pm 96$ & $995 \pm 78$ \\
\hline HEART & $1.8 \pm 0.0$ & $1043 \pm 132$ & $602 \pm 71$ & $456 \pm 79$ & $338 \pm 25$ \\
\hline LIVER & $9.8 \pm 0.2$ & $6479 \pm 247$ & $9307 \pm 813$ & $14287 \pm 944$ & $14555 \pm 932$ \\
\hline SPLEEN & $4.6 \pm 0.1$ & $2578 \pm 179$ & $3150 \pm 269$ & $4443 \pm 395$ & $4104 \pm 356$ \\
\hline KIDNEY & $19.1 \pm 0.2$ & $1647 \pm 147$ & $2586 \pm 129$ & $5033 \pm 625$ & $7379 \pm 563$ \\
\hline BRAIN & $0.3 \pm 0.0$ & $189 \pm 25$ & $90 \pm 12$ & $64 \pm 18$ & $79 \pm 24$ \\
\hline TESTIS & $0.8 \pm 0.0$ & $297 \pm 16$ & $375 \pm 28$ & $613 \pm 38$ & $591 \pm 61$ \\
\hline
\end{tabular}

Table 4.2. Comparison of cadmium levels in CdTe-QD- and $\mathrm{CdCl}_{2}$-treated blood and tissues. Mice were treated with $6 \mathrm{mg} / \mathrm{kg}$ bw of CdTe-QDs or $1.95 \mathrm{mg} / \mathrm{kg}$ bw of $\mathrm{CdCl}_{2}$ which contains an equivalent cadmium concentration, and their blood and tissues examined at $24 \mathrm{~h}$ post-exposure.

\begin{tabular}{|c|c|c|c|}
\hline Sample & Control & CdTe & $\mathrm{CdCl}_{2}$ \\
\hline BLOOD & $0.0 \pm 0.0$ & $10146 \pm 151$ & \pm 13 \\
\hline LUNG & $2.1 \pm 0.0$ & $6269 \pm 785$ & $615 \pm 72$ \\
\hline HEART & $1.7 \pm 0.0$ & $1832 \pm 247$ & $234 \pm 12$ \\
\hline LIVER & $5.2 \pm 0.3$ & $18761 \pm 612$ & $12531 \pm 1139$ \\
\hline SPLEEN & $4.6 \pm 0.1$ & $10189 \pm 943$ & $587 \pm 61$ \\
\hline KIDNEY & $12.3 \pm 0.5$ & $6909 \pm 674$ & $3458 \pm 429$ \\
\hline BRAIN & $0.7 \pm 0.1$ & \pm 25 & \pm 4 \\
\hline
\end{tabular}




\subsubsection{Detection of CdTe-QDs in blood and tissues}

Confocal microscopy and spectrofluorometry were used to confirm the presence of CdTe-QDs in the blood and tissues. The blood and tissues showed high background fluorescence intensities when measured by spectrofluorometry and confocal microscopy due to tissue auto-fluorescence. However, at the same tissue concentrations, the homogenates of the lung, heart, liver, spleen, kidney, brain and testis from CdTe-QDtreated mice showed significantly greater fluorescence intensity than the control homogenates (Figure 4.3.). Confocal microscopy of blood and tissue sections showed fluorescent bright green structures that were not observed in the control tissues, speculating the presence of CdTe-QDs in the blood, lung, liver, spleen, and kidney (Figures 4.4.A, B, D, E, and F) but not in the heart, brain and testis (Figures 4.3.C, G, and $\mathrm{H})$.

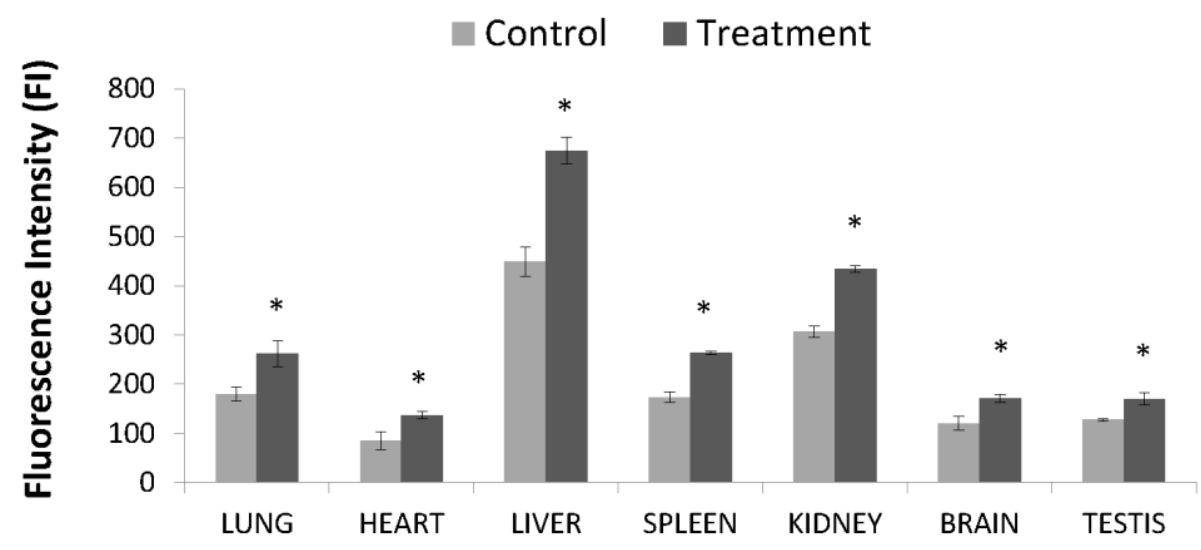

Figure 4.3. Detection of CdTe-QDs in tissue homogenates using spectrofluorometry. The fluorescence intensity was measured at Ex. of $340 \mathrm{~nm}$ and Em. of $550 \mathrm{~nm}$. Mice were treated with $10 \mathrm{mg} / \mathrm{kg}$ bw of CdTe-QDs for $24 \mathrm{~h}$. The asterisks (*) indicate statistically significant differences compared to the control group $(\mathrm{p}<0.05)$. 

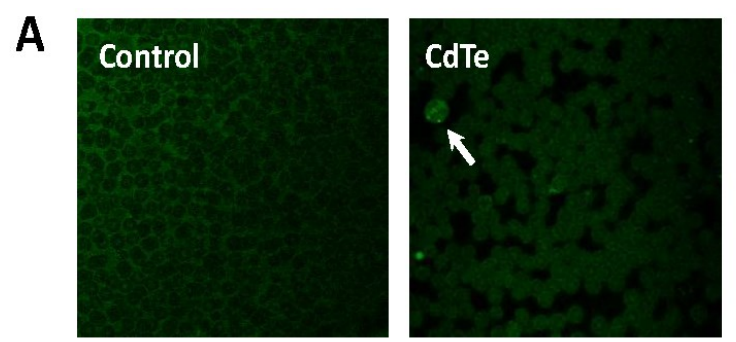

C
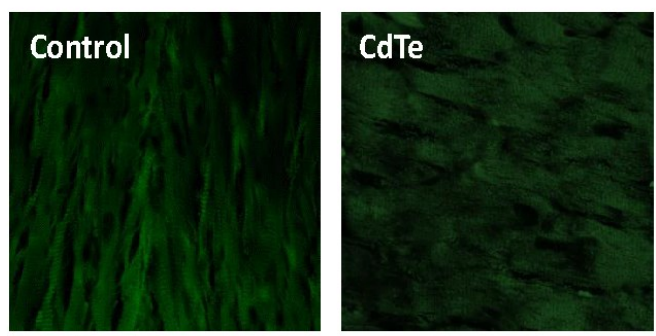

$\mathrm{E}$

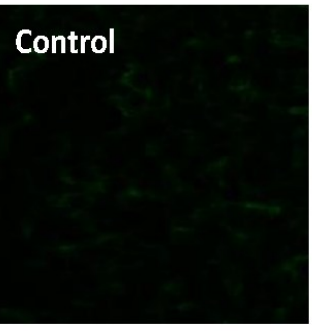

G

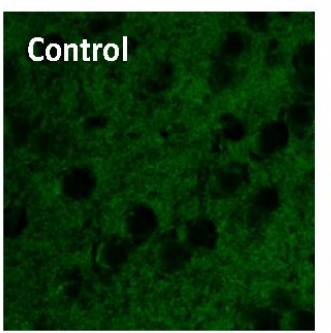

B
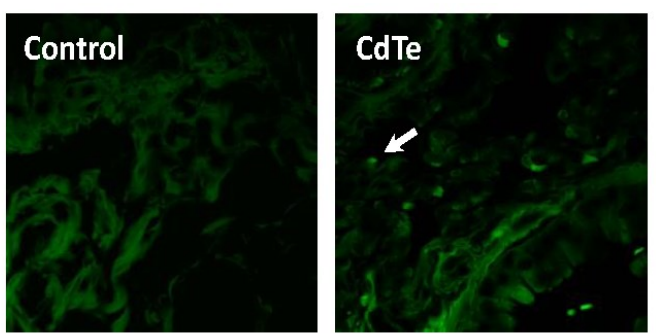

D
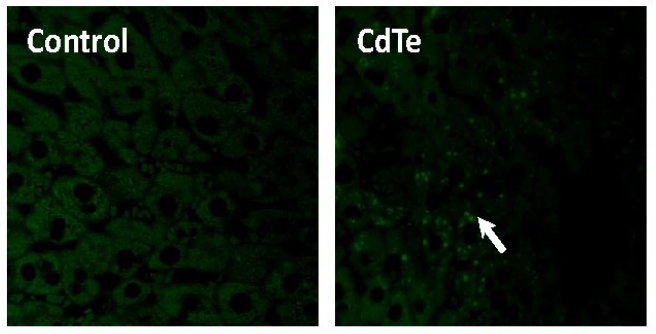

$\mathbf{F}$
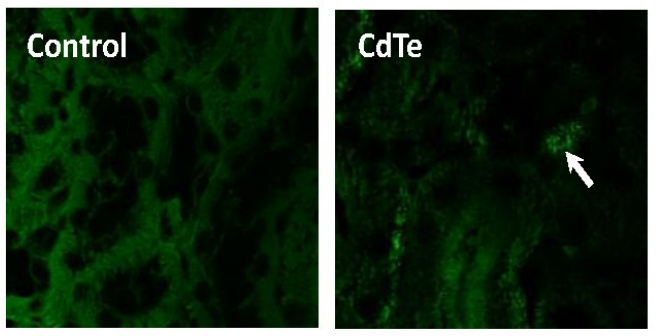

H
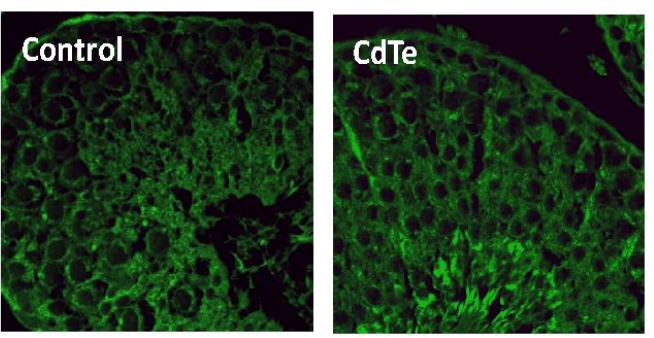

Figure 4.4. Detection of CdTe-QDs in blood smears and tissue sections $24 \mathrm{~h}$ after mice were treated with CdTe-QDs (10 mg/kg bw), using confocal microscopy (Ex. $340 \mathrm{~nm}$, Em. 515/530 nm). (A) blood, (B) lung, (C) heart, (D) liver, (E) spleen, (F) kidney, (G) brain, $(\mathbf{H})$ testis. The blood smears and tissue sections exhibited a green background and CdTe-QDs appeared as brighter green dots as indicated by the arrows. 


\subsubsection{Effects of CdTe-QDs on behaviour and physical appearance of mice}

At $24 \mathrm{~h}$, the high doses $(6-10 \mathrm{mg} / \mathrm{kg}$ bw) of CdTe-QDs caused general malaise of the mice. Treated mice appeared slow in activity and showed signs of distress including mild irregular respiration, mild dehydration, lethargy, ruffled fur, and hunched posture. Treatment with $10 \mathrm{mg} / \mathrm{kg}$ bw CdTe-QDs additionally led to an approximate $10 \%$ body weight loss (data not shown). Lower CdTe-QD doses $\left(0.4-5 \mathrm{mg} / \mathrm{kg}\right.$ bw) and $\mathrm{CdCl}_{2}$ did not cause changes in animal physical activity and appearance.

\subsubsection{Effects of CdTe-QDs on tissue morphology}

The necropsies revealed visible macroscopic changes in the liver and spleen of CdTe-QD-treated mice. At a dose of $6 \mathrm{mg} / \mathrm{kg}$ bw or higher, these organs appeared dark red in color compared to those from the control (data not shown). Hematoxylin and eosin (H\&E) stained sections from these tissues showed areas hemorrhage and necrosis (Figures 4.5. and 4.6.). Specifically, treatments of CdTe-QDs at $6 \mathrm{mg} / \mathrm{kg}$ bw or higher induced liver hemorrhage that was observed in the periportal areas of the hepatic lobules accompanied with apoptotic and necrotic liver cells in those areas (Figures 4.5.B, C, F, and G). Similarly, bleeding and disruption normal structure of marginal regions in spleen were observed with CdTe-QD treatments at $6 \mathrm{mg} / \mathrm{kg}$ bw or higher (Figures 4.6.B, C, F and G). There were no changes in the appearance and morphology of the lung, heart, kidney, brain and testis (data not shown). Mice treated with $\mathrm{CdCl}_{2}$ at an equivalent cadmium concentration to $6 \mathrm{mg} / \mathrm{kg}$ bw CdTe-QDs did not induce alteration in macroscopic and histological changes in the liver and spleen (Figures 4.5.D and H; Figures 4.6.D and $\mathrm{H}$ ) or in other tissues examined (data not shown). 

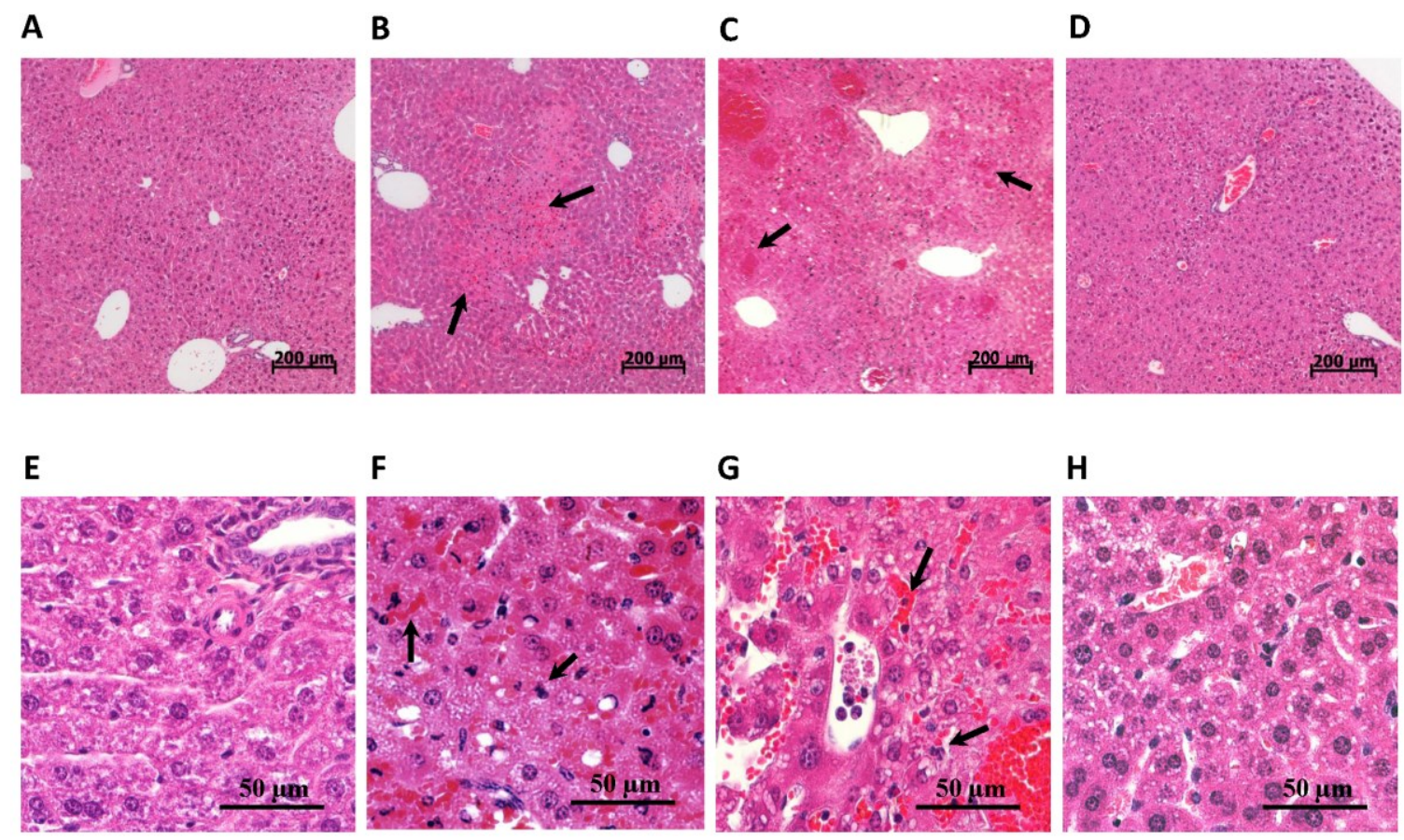

Figure 4.5. Tissue histological images of the livers stained with H\&E, obtained from mice treated with saline (A and $\mathbf{E}), 6 \mathrm{mg} / \mathrm{kg}$ bw CdTe-QDs $(\mathbf{B}$ and $\mathbf{F}), 10 \mathrm{mg} / \mathrm{kg}$ bw CdTe-QDs ( $\mathbf{C}$ and $\mathbf{G})$, and $1.95 \mathrm{mg} / \mathrm{kg}$ bw $\mathrm{CdCl}_{2}$ (D and $\left.\mathbf{H}\right)$. The H\&E stained sections were examined with a light microscopy at magnification of 10x (A, B, C, and D) and 60x $(\mathbf{E}, \mathbf{F}, \mathbf{G}$, and $\mathbf{H})$. The arrows indicate the areas of hemorrhage, apoptotic and necrotic events. 
A

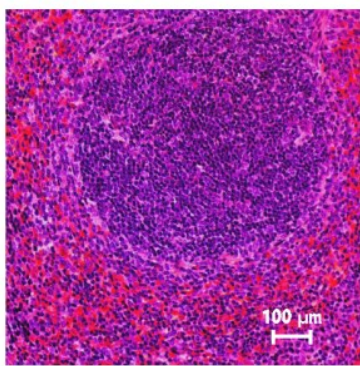

E

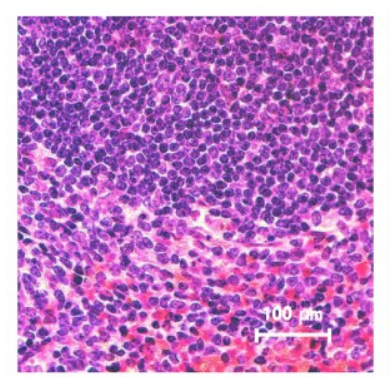

B

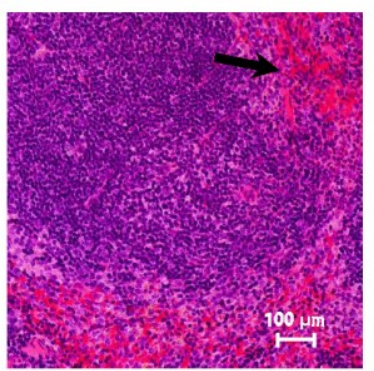

$\mathbf{F}$

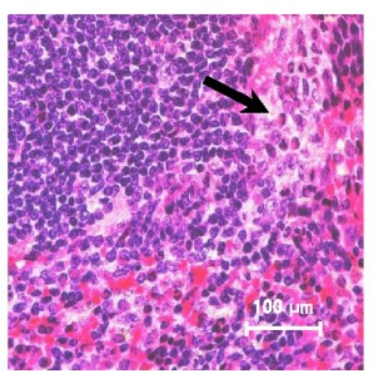

C

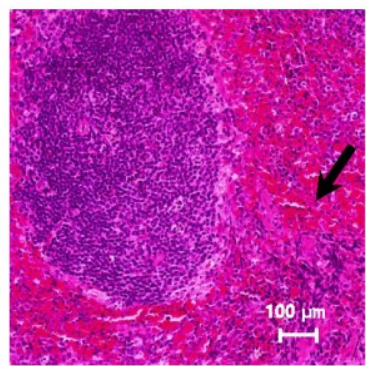

G

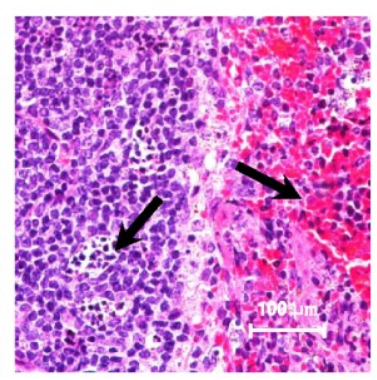

D

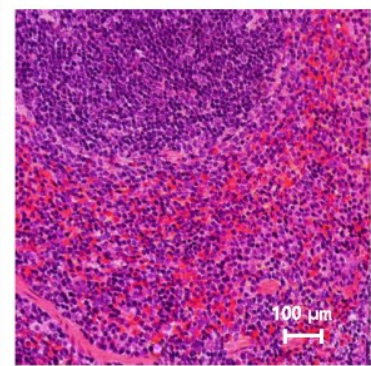

H

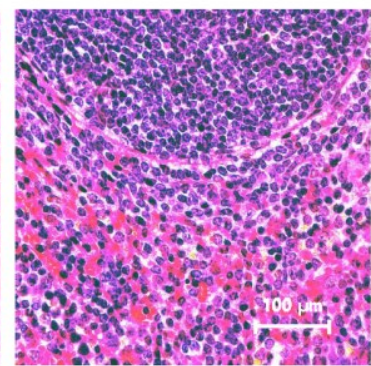

Figure 4.6. Tissue histological images of the spleen stained with H\&E, obtained from mice treated with saline (A and E), 6mg/kg bw CdTe-QDs ( $\mathbf{B}$ and $\mathbf{F}$ ), $10 \mathrm{mg} / \mathrm{kg}$ bw CdTe-QDs (C and $\mathbf{G})$, and $1.95 \mathrm{mg} / \mathrm{kg}$ bw $\mathrm{CdCl}_{2}$ (D and $\left.\mathbf{H}\right)$. The H\&E stained sections were examined with a light microscopy at magnification of 20x (A, B, C, and D) and 40x $(\mathbf{E}, \mathbf{F}, \mathbf{G}$, and $\mathbf{H})$. The arrows indicate the areas of hemorrhage and necrotic events.

\subsubsection{Changes in biochemical parameters}

Biochemical tests of serum samples showed that, at the high doses, CdTe-QDs induced a decrease in albumin level and increases in ALT, AST, and total bilirubin levels (Figures 4.7.A, B, C, and D). However, CdTe-QDs did not cause change in the level of creatinine (Figures 4.7.E). Treatment with $\mathrm{CdCl}_{2}$ (containing an equivalent concentration 
of cadmium to $6 \mathrm{mg} / \mathrm{kg}$ bw) did not cause changes in the levels of test biochemical biomarkers (Figures 4.7. A, B, C, and D).

A

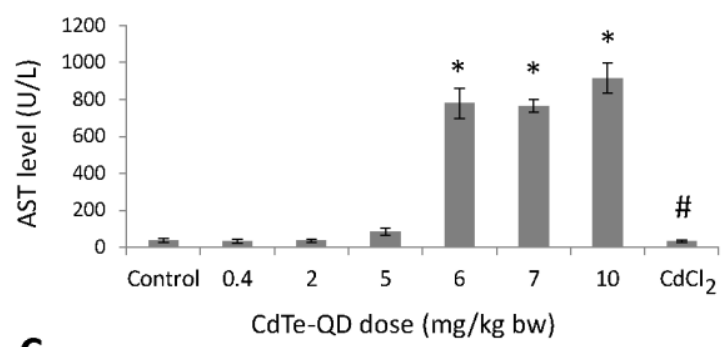

C

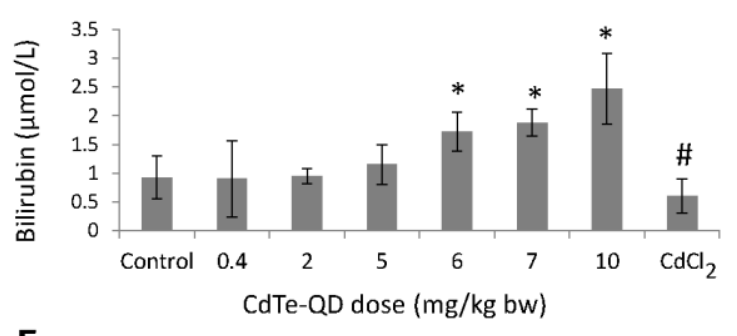

E

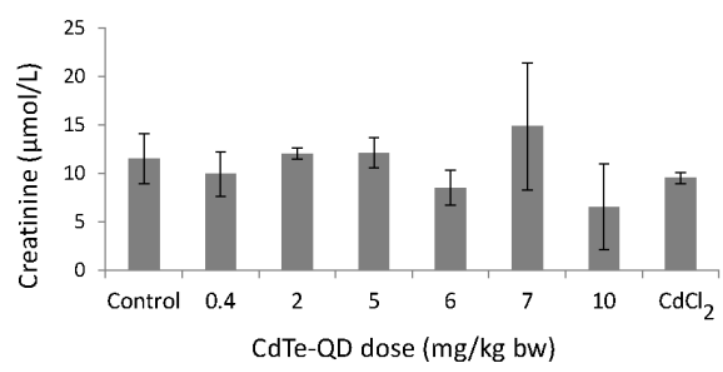

B

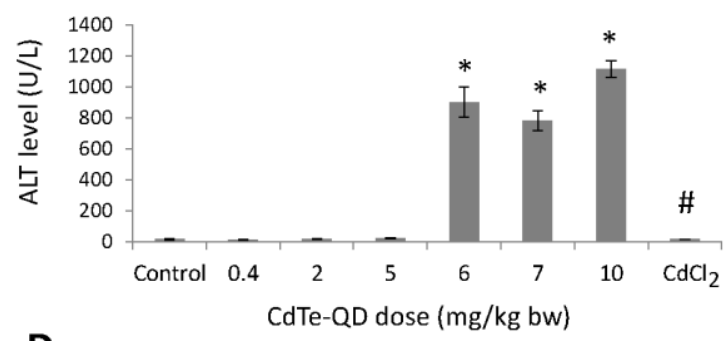

D

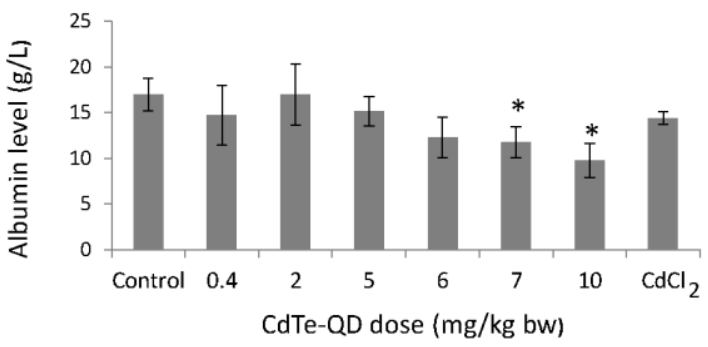

Figure 4.7. Biochemistry resuls for (A) aspartase transaminase (AST), (B) alanine aminotransferase (ALT), (C) total bilirubin, (D) albumin, and (E) creatinine in serum samples from the control or saline-, $\mathrm{CdTe}-\mathrm{QD}-$ and $\mathrm{CdCl}_{2}$ - treated mice. Serum samples were obtained at $24 \mathrm{~h}$ after mice were treated with different concentrations of CdTe-QDs and $1.95 \mathrm{mg} / \mathrm{kg}$ bw $\mathrm{CdCl}_{2}$, which contains an equivalent cadmium concentration to 6 $\mathrm{mg} / \mathrm{kg}$ bw CdTe-QDs. The asterisks (*) indicates statistically significant differences compared to the control $(\mathrm{p}<0.05)$. The number $(\#)$ sign indicates statistically significant difference compared to the CdTe-QD-treated group $(\mathrm{p}<0.05)$. 


\subsubsection{Changes in haematology}

Haematology analysis showed that at $24 \mathrm{~h}$ post-exposure, CdTe-QD treatment resulted in an increased number of white blood cells (WBC) at the highest dose (10 $\mathrm{mg} / \mathrm{kg} \mathrm{bw}$ ) (Figure 4.8.A). However, increases in the percentages of blood monocytes and neutrophils were observed at $5 \mathrm{mg} / \mathrm{kg}$ bw (Figures 4.8. B and C). At $24 \mathrm{~h}$ postexposure, CdTe-QDs also resulted in a reduction in the number platelets at $5 \mathrm{mg} / \mathrm{kg}$ bw (25\%), $6 \mathrm{mg} / \mathrm{kg}$ bw (60\%), $7 \mathrm{mg} / \mathrm{kg}$ bw (80\%), and $10 \mathrm{mg} / \mathrm{kg}$ bw (100\%) (Figure 4.8.D). However, in mice treated with $5 \mathrm{mg} / \mathrm{kg}$ bw, the platelet numbers appeared to recover to the levels that were comparable to that of the control at $3 \mathrm{~d}$ and $1 \mathrm{w}$ (Figure 4.8.E). Blood smear stains also revealed the increase in the number of reticulocytes (immature red blood cells) in the blood of mice treated with $10 \mathrm{mg} / \mathrm{kg}$ bw CdTe-QDs (Figure 4.8.F). $\mathrm{CdCl}_{2}$ also caused an increase in the percentage of monocytes, but did not result in any observable changes in other haematology parameters (Figures 4.8.A and B). 
A

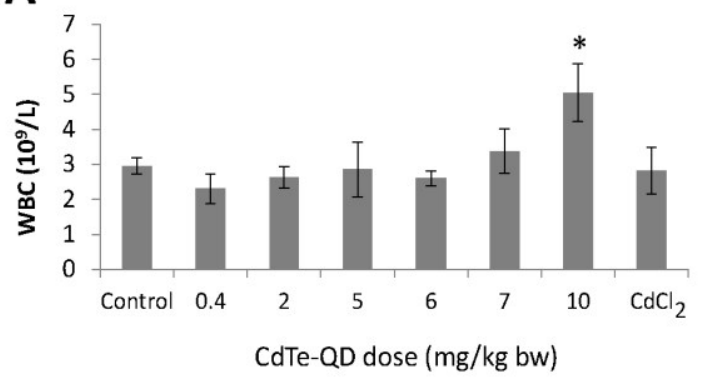

B

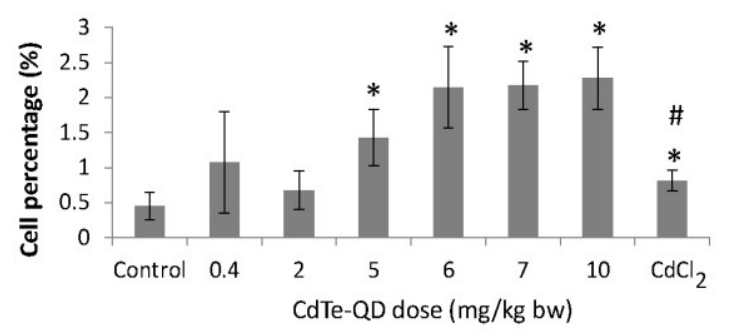

C

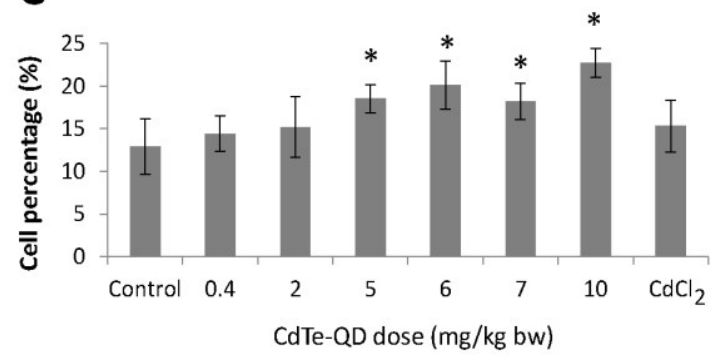

D

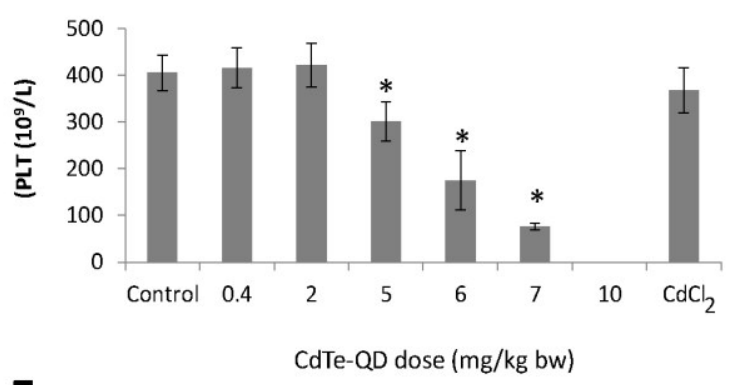

$\mathbf{E}$

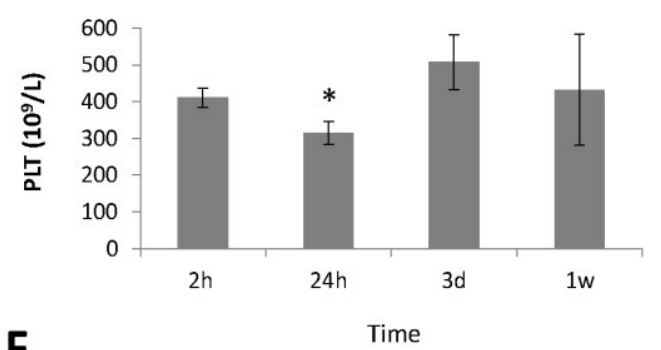

$\mathbf{F}$
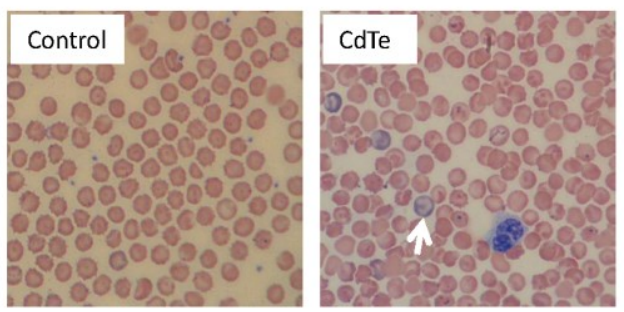

Figure 4.8. Haematology analysis of saline-, $\mathrm{CdTe}-\mathrm{QD}-$ and $\mathrm{CdCl}_{2}$-treated mice. (A) White blood counts (WBC), (B) percentage of monocytes, $(\mathbf{C})$ percentage of neutrophils, (D) platelet counts (PLT) at $24 \mathrm{~h}$ after mice were treated with saline, different concentrations of CdTe-QDs, and $1.95 \mathrm{mg} / \mathrm{kg}$ bw of $\mathrm{CdCl}_{2}$ (containing an equivalent of cadmium concentration to $6 \mathrm{mg} / \mathrm{kg}$ bw of CdTe-QDs). (E) Platelets (PLT) counts in mice treated with $5 \mathrm{mg} / \mathrm{kg}$ bw CdTe-QDs for different post-exposure sampling times. The asterisks $(*)$ indicates statistically significant differences compared to the control $(\mathrm{p}<0.05)$. (F) The blood smears were stained with Wright's stain. Mice were treated with $10 \mathrm{mg} / \mathrm{kg}$ bw CdTe-QDs for $24 \mathrm{~h}$. Reticulocytes are indicated by the arrow. 


\subsubsection{Pro-inflammation and acute phase responses}

CdTe-QD treatments resulted in changes in the level of the acute phase response protein, SAA. At $5 \mathrm{mg} / \mathrm{kg}$ bw or higher, CdTe-QDs induced a significant increase in serum SAA level (Figures 4.9.A and B). Treatment of $\mathrm{CdCl}_{2}$ also resulted in a significantly elevated SAA level (Figure 4.9.A). However, $\mathrm{CdCl}_{2}$ effect was much less than that of $6 \mathrm{mg} / \mathrm{kg}$ bw CdTe-QDs that contains an equivalent cadmium concentration (Figure 4.9.A).

In addition, both $\mathrm{CdTe}-\mathrm{QDs}$ and $\mathrm{CdCl}_{2}$ altered the levels of several proinflammatory cytokines in serum. Specifically, starting at $0.4 \mathrm{mg} / \mathrm{kg}$ bw, CdTe-QDs induced significant increase in IL-6 level. Elevations of KC (keratinocyte chemoattractant), IL-12 (p70), and TNF- $\alpha$ levels were observed with treatments of CdTeQDs at $5 \mathrm{mg} / \mathrm{kg}$ bw or higher. (Figures 4.10.A, B, C, and D). However, significant drops in IL-12 (p70) and TNF- $\alpha$ occurred at highest doses, 7 and $10 \mathrm{mg} / \mathrm{kg}$ bw (Figure 4.10.B). Treatments with $\mathrm{CdCl}_{2}$ also resulted in increased IL-6, KC, and TNF- $\alpha$ (Figures 4.10.A and B). The elevations of these cytokines were also observed in mice treated with 5 $\mathrm{mg} / \mathrm{kg}$ bw of CdTe-QDs at longer time points, $3 \mathrm{~d}$ and $1 \mathrm{w}$ (Figures 4.10.C and D). 
A

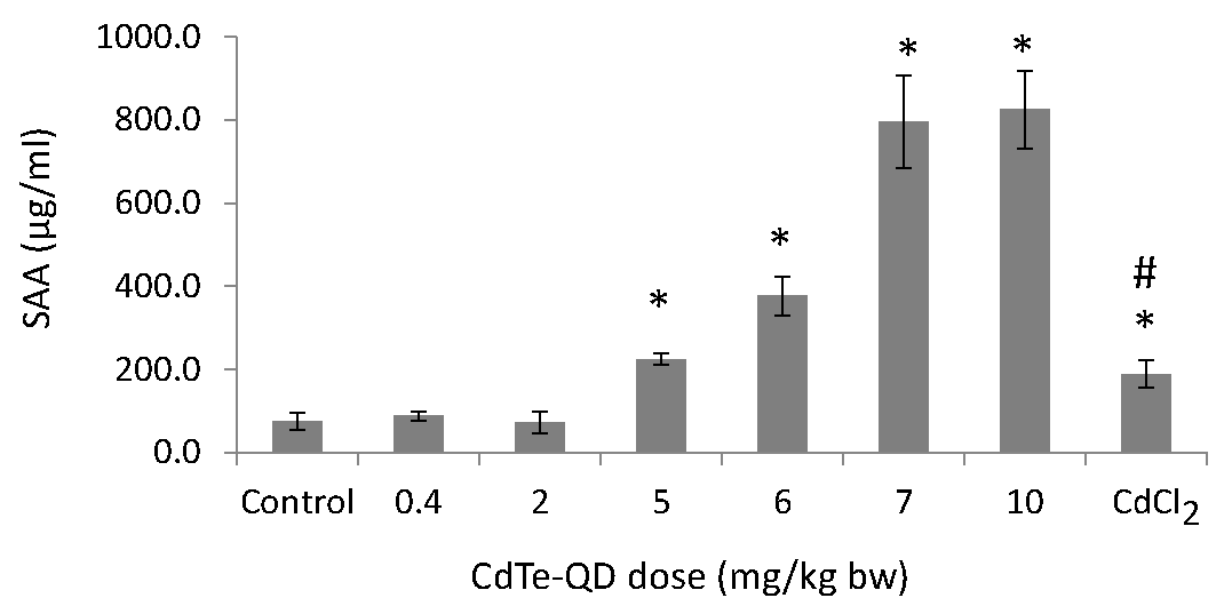

B

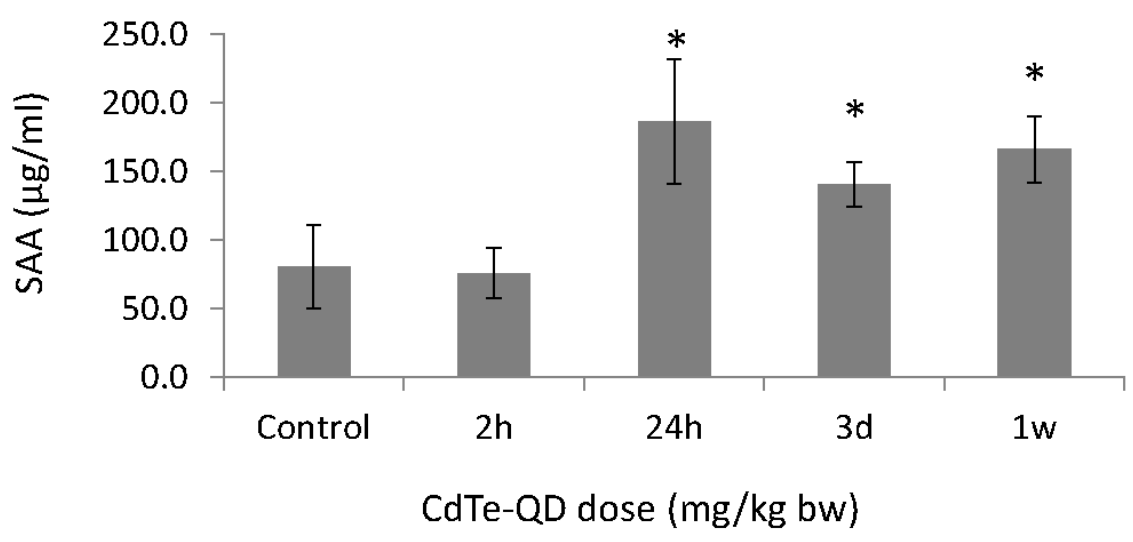

Figure 4.9. Effects of CdTe-QDs and $\mathrm{CdCl}_{2}$ on serum amyloid A (SAA). (A) SAA levels $24 \mathrm{~h}$ after mice were treated with saline, different concentrations of CdTe-QDs, and $1.95 \mathrm{mg} / \mathrm{kg}$ bw of $\mathrm{CdCl}_{2}$, which contains the equivalent cadmium concentration as that of $6 \mathrm{mg} / \mathrm{kg}$ bw CdTe-QDs. (B) SAA levels in mice treated with $5 \mathrm{mg} / \mathrm{kg}$ bw CdTeQDs for different time points. The asterisks (*) indicates statistically significant differences compared to the control $(\mathrm{p}<0.05)$. The number (\#) sign indicates statistically significant difference compared to the $6 \mathrm{mg} / \mathrm{kg}$ bw CdTe-QD-treated group $(\mathrm{p}<0.05)$. 
A

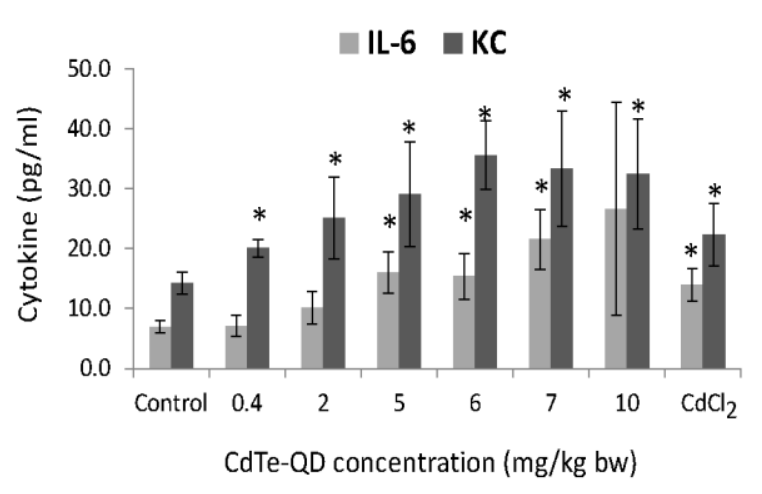

C

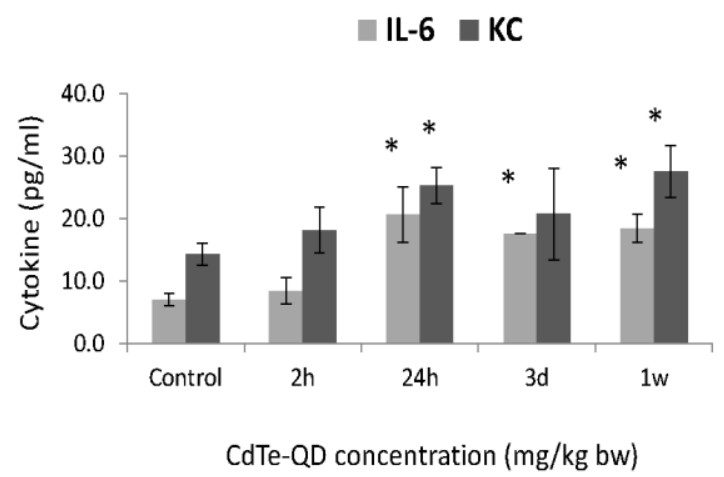

B

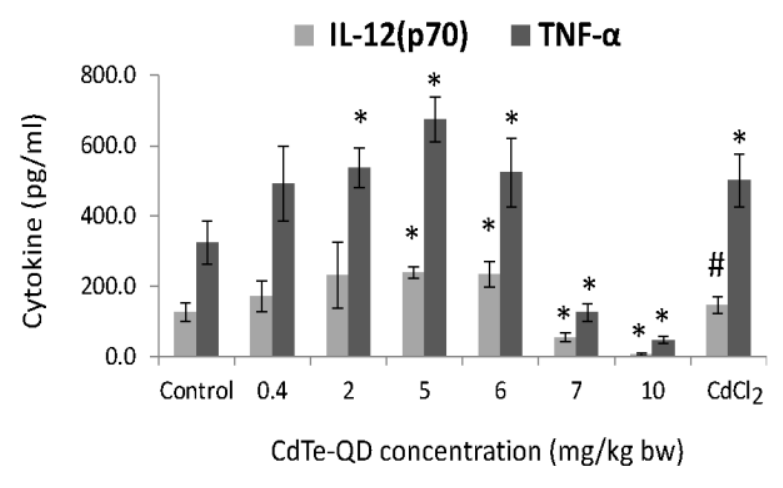

D

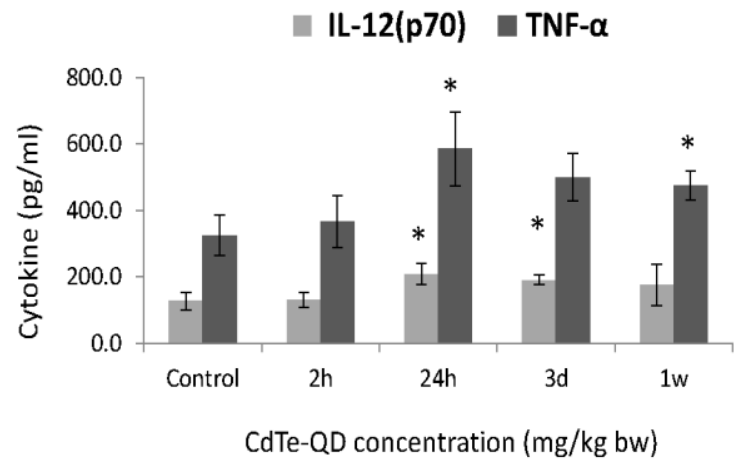

Figure 4.10. Effects of CdTe-QDs and $\mathrm{CdCl}_{2}$ on selected serum cytokines. Levels of (A) IL-6 and KC, (B) IL-12 (p70) and TNF- $\alpha$ in mice treated with saline, different concentrations of CdTe-QDs, and $1.95 \mathrm{mg} / \mathrm{kg}$ bw of $\mathrm{CdCl}_{2}$, which contains an equivalent cadmium concentration to $6 \mathrm{mg} / \mathrm{kg}$ bw CdTe-QDs, for $24 \mathrm{~h}$. Levels of (C) IL-6 and KC, (D) IL-12 (p70) and TNF- $\alpha$ in mice treated with $5 \mathrm{mg} / \mathrm{kg}$ bw CdTe-QDs for different time points. The asterisks $(*)$ indicates statistically significant differences compared to the control $(\mathrm{p}<0.05)$. The number $(\#)$ sign indicates statistically significant difference compared to the CdTe-QD-treated group $(\mathrm{p}<0.05)$. 


\subsection{Discussion}

Due to the increases in the production and use of cadmium QDs, as well as their potential for applications in biology and medicine, more information on their behaviour and biological effects is necessary for safe human use. This study examined the distribution of CdTe-QDs and their toxic effects in BALB/c mice after intravenous injection. In terms of the study design, BALB/c mice were chosen as the animal model for the study as this strain has been employed in several in vivo QD studies and widely used in toxicological studies of drugs and xenobiotics (You et al., 2006). In addition, they are readily available and easy to handle and manage. Similarly, this study employed intravenous injection, which has been considered a relevant exposure route for CdTeQDs because these NPs have the potential for medical imaging for diagnostics and therapeutics. Furthermore, the doses used were within the range of those used for in vivo applications of quantum dots (Ballou et al., 2004; Gao et al., 2004; Geys et al., 2008) and comparable to reported doses from other in vivo studies (Haque et al., 2012; So et al., 2006; Su et al., 2011).

The distribution of CdTe-QDs in the blood and tissues was first observed by examining the concentrations of cadmium in the tissues using ICP-MS. Our results showed that CdTe-QDs distributed to the lung, heart, liver, spleen, kidney, brain and testis. The liver, spleen and kidney exhibited greatest levels of cadmium, suggesting these tissues are preferential sites of CdTe-QD accumulation. However, the greatest accumulation of CdTe-QDs over time was in the liver, suggesting that this organ is the primary target of these NPs after being administered into the animals. Our finding agreed with previous studies that also showed that after tail vein intravenous injection, various 
types of QDs accumulated in the liver and spleen, and their excretion occurred through the kidney (Yang et al., 2007; Cai et al., 2007; Lin et al., 2008; Chen et al., 2008; Haque et al., 2012; Su et al., 2011; Tiwari et al., 2011). It has been suggested that the uptake of NPs into the reticuloendothelial (RE) tissues, such as liver, spleen, and lungs, was due to the nonspecific NP clearance from the blood by cells of the mononuclear phagocyte system (Fischer et al., 2006). However, similar to the report by Haque et al. (2012), the distribution of CdTe-QDs in the lungs in the current study was much less than those in the liver and spleen and the level of CdTe-QDs in the lungs decreased over the time after $24 \mathrm{~h}$. This suggests that the specific distribution of CdTe-QDs to the liver and spleen is not consistent with the RE system and could be a result of other factors (Haque et al., 2012). The observed accumulation of CdTe-QDs in the liver and spleen could be due to the site of the injection which was at the tail vein in this present study and that of Haque and colleagues. By contrast, Salykina et al. (2011), who used the same MPA-coated QDs (as in our study), reported that the primary accumulation of these QDs was in lung since their QDs were injected the jugular vein. Furthermore, tissue distribution of QDs has been shown to depend on the surface coating of these NPs (Gao et al., 2004; Ballou et al., 2004; Fischer et al., 2006). It has been reported that negatively charged QDs such as MPA-coated QDs used in this study may interact and adsorb proteins in the blood (Salykina et al., 2008). This protein binding plays a key role in delivering NPs from plasma into the liver, which appeared to be the main site of accumulation of CdTe-QDs in this study, as the role of this organ in the metabolism and degeneration of a multitude of xenobiotics is well described (Sturgill et al., 1997). 
To compare the distribution of CdTe-QDs to that of $\mathrm{CdCl}_{2}$, injection of $\mathrm{CdCl}_{2}$ containing an equivalent cadmium concentration to one of CdTe-QD doses was performed. Based on the cadmium concentration in the blood and examined tissues measured by ICP-MS at $24 \mathrm{~h}$ post-exposure, the distribution pattern of $\mathrm{CdCl}_{2}$ appeared to be different from that of CdTe-QDs. At $24 \mathrm{~h}$ post-exposure, the cadmium level from $\mathrm{CdCl}_{2}$ was very low in the blood while a certain level of cadmium from CdTe-QDs still remained in the blood. The level of cadmium from $\mathrm{CdCl}_{2}$ appeared greatest in the liver and kidney, but was very low in spleen and other tissues. Although an equivalent cadmium concentration was used for both CdTe-QDs and $\mathrm{CdCl}_{2}$, in all tissues, the levels of cadmium from $\mathrm{CdCl}_{2}$ appeared significant lower than those from CdTe-QDs suggesting $\mathrm{CdCl}_{2}$ has a shorter retention time in the animal than CdTe-QDs and its pharmacokinetics appeared to be different from that of CdTe-QDs. Our findings agreed with previous reports showing that cadmium accumulated primarily in the liver and kidney after intravenous injection (Byerrum et al., 1957; Berlin and Ullberg, 1963; Shank et al., 1977). It has been reported that cadmium, after being introduced into systemic circulation, binds to albumin in the blood plasma, transports to the liver where the complex cadmium-metallothionein is formed and transported to the kidneys for excretion (Jin et al., 1998). Our findings also align with a previous study by Yeh et al. (2011), who showed that QDs and $\mathrm{CdCl}_{2}$ had very different kinetics in distribution and metabolism after intravenous injection. Similar to our results, in this previous study, the authors found that QDs were retained in plasma and organs longer than $\mathrm{CdCl}_{2}$, and that QDs could be detected in the brain during early exposure (Yeh et al., 2011). 
Characterization and detection of NPs within biological systems have been challenging in nanotoxicological research due to limited methods and techniques for measurement and detection of NPs in tissues. In this study, the fluorescent property of CdTe-QDs permitted detection in the blood and tissues using confocal microscopy and spectrofluorometry. Control tissue and blood auto-fluorescence appeared relatively high, however compared to tissues of CdTe-QD-treated mice significantly greater fluorescence was observed, suggesting the presence of CdTe-QDs in these tissues. These results somewhat corresponded to tissue levels of cadmium measured by ICP-MS. Confocal microscopy demonstrated the presence of CdTe-QDs in the blood, lung, liver, spleen, kidney, but not in the brain, heart and testes, suggesting that confocal microscopy has limitations associated with structural tissue interference and auto-fluorescence.

Kato and colleagues (2010) demonstrated that $\mathrm{CdSe} / \mathrm{ZnS}(13.5 \mathrm{~nm})$ were able to cross the blood brain barrier QDs as evidenced by the presence of these NPs in the parenchyma of the brain at $6 \mathrm{~h}$ after intraperitoneal administration (Kato et al., 2010). In our study, the presence of CdTe-QDs in the brain was detected by ICP-MS and spectrofluorometry. Since our particles are less than $5 \mathrm{~nm}$ (by DLS), it is possible that some CdTe-QDs crossed the barrier. However, levels detected in the brain using these techniques were minimal compared to those detected in the preferential sites of accumulation, and CdTe-QDs were not observed in the brain sections using confocal microscopy. In addition, the levels of cadmium from CdTe-QDs in the brain decreased over the time. These findings suggest that CdTe-QDs detected in the brain were present in the blood vessels, but detailed distribution patterns of CdTe-QDs within the brain need to be further investigated. 
CdTe-QDs were also observed in the heart, and its levels decreased over time suggesting a similar kinetic to that in the blood. Interestingly, the levels of CdTe-QDs in the testes increased over the time suggesting these organs may be an important tissue for chronic exposures. Certainly a more detailed study of CdTe-QDs distribution in these tissues needs to be further investigated.

Further examination of the preferential sites of accumulation demonstrated pathological and toxicological effects. Histological examination revealed changes in the liver and spleen tissue morphology, but not other tissues examined. Liver and spleen showed haemorrhage at highest doses. The liver bleeding was localized in the periportal areas of the lobules, which may have resulted from damaged endothelial cells of the portal vein resulting in leakage of red blood cells in these areas. Furthermore CdTe-QDs induced significantly increased ALT, AST, and bilirubin and decreased albumin in the serum, which are known biomarkers of liver injury (Daniel and Marshall, 1999). Our study supports previous studies showing liver toxicity caused by QDs in vivo. Tiwari et al. (2011) reported increased serum AST and ALT levels induced by intravenous injection of QDs in rats indicating liver injury. Similarly, a study by Lin et al. (2011) reported that reported that intravenous injection of $\mathrm{Cd} / \mathrm{Se} / \mathrm{Te}-\mathrm{based} \mathrm{QD} 705$ in mice resulted in significant elevation in ALT and AST activities at 12 weeks and 16 weeks post-exposure. In this present study, although the kidney was one of the sites of QD accumulation, there was no observable alteration in its histopathology. In addition, biochemical analyses showed no changes in the level of creatinine, which is an important indicator of renal injury. $\mathrm{CdCl}_{2}$, containing an equivalent cadmium concentration to a CdTe-QDs dose that caused liver injury, did not cause changes either in the liver or the 
kidney in our study. This may be due to either the difference in in vivo pharmacokinetics or the mechanisms of action between QDs and $\mathrm{CdCl}_{2}$. In addition, the $\mathrm{CdCl}_{2}$ dose used here is much lower than the toxic dose to mice as reported in previous studies (Ryan, et al., 1982; Kotsonis and Klaassen, 1977). Our results also suggested that CdTe-QDs are more toxic than $\mathrm{CdCl}_{2}$ when being injected into the animal and this finding agrees with our previous in vitro studies (Nguyen et al., 2013b; Nguyen et al., 2015).

Systemic effects of CdTe-QDs were also investigated. Increased numbers of WBC were observed following exposure to the highest test dose. Specifically, the incresases in blood monocytes an neutrophils were detected, indicating an immunological response. In addition, Wright stain of blood revealed an increase in the number of reticulocytes, suggesting acute anemia caused by hemorrhage. This result corroborated the hepatic hemorrhage observed from histological analysis. CdTe-QDs also caused significant dose-dependent reduction in the number platelets. Cadmium QDs such as $\mathrm{CdSe} / \mathrm{ZnS}$ have been shown to cause aggregation of platelets, as well as a drop in the circulating platelet count (Geys et al., 2008). In this previous study, the authors suggest that activation of platelets with aggregates retained in the pulmonary microvasculature eventually lead to pulmonary thrombosis. In vitro, the authors of this previous study showed that QDs enhanced adenosine-5' -diphosphate-induced platelet aggregation (Geys et al., 2008). In our present study, although the platelet number significantly decreased, there were no effects observed in the lungs as these organs were not the main targets of our test CdTe-QDs. The difference between our study and Geys' study could be due to the different types of QDs used. Furthermore, the platelet number in animals and human is control by a protein called thrombopoietin, which is produced mainly in the liver (Kato 
et al., 1995). It has been reported that a decrease in the platelet number was associated with both acute and chronic hepatitis due to either impaired synthesis or increased degradation of thrombopoietin (Narita et al., 2003; Rios et al., 2005). Therefore, in our present study, the decreased platelet number induced by CdTe-QDs could be a result of the combination of platelet aggregation, as discussed above, and liver injury that led to a decrease in the synthesis of thrombopoietin. $\mathrm{CdCl}_{2}$ did not cause any observable changes in haematology, and again this is due to the fact that the concentration of $\mathrm{CdCl}_{2}$ used in this study was much less than the $\mathrm{CdCl}_{2}$ concentrations that are toxic in animals (Kotsonis et al., 1977; Kuester et al., 2002).

Serum cytokine and SAA levels were also employed as indicators of systemic effects of CdTe-QDs. SAA are a family of acute phase proteins and expressed in response to inflammatory stimuli. Their hepatic expression is regulated by the proinflammatory cytokines IL-1 $\beta$, IL-6, and TNF- $\alpha$ (Uhlar and Whitehead, 1999). Thus, in the current study the increased levels of SAA may result from the elevated IL-6 and TNF- $\alpha$. It has been shown that during liver injury, resident Kupffer cells and monocyte/macrophages initiate an immune response by producing pro-inflammatory cytokines such as TNF- $\alpha$ and IL-6 that initiate the acute-phase response and inflammation. Such acute inflammation is characterized by the rise in concentration of numerous plasma acute-phase proteins including SAA (Billiar et al., 1992). The observed inflammation induced by CdTe-QDs agrees with the increase in the number of WBC, monocytes, and neutrophils in the blood of the treated mice. It is worthy to note that the elevations of SAA and cytokines, as well as monocytes and neutrophils occurred at the threshold or lower doses. In addition, the effects on these endpoints were also 
observed with $\mathrm{CdCl}_{2}$. This indicates that $\mathrm{SAA}$ and serum cytokines are sensitive biomarkers of systemic effects of QDs and $\mathrm{CdCl}_{2}$ and inflammation appeared to be early events of CdTe-QD toxicity. In this study, the increases in SAA and serum cytokine levels were also observed at longer exposures, and these increases can be explained by the accumulation of QDs in the liver and spleen over the time as previously discussed. At the highest doses of CdTe-QDs, the levels of IL-12(p70) and TNF- $\alpha$ decreased and this might be associated with excessive injuries of target organs, including liver and spleen and the detailed mechanisms of these inhibitory effects at high doses need to be further investigated.

In summary, the study investigated the biodistribution and toxic effects of MPAcoated CdTe-QDs in BALB/c mice. Our results showed the NPs distributed to all examined tissues, but the liver is the primary target. CdTe-QDs caused hepatic and splenic injury and induced systemic effects. Further studies are required to determine the underlying mechanisms of these effects. At an equivalent cadmium concentration, $\mathrm{CdCl}_{2}$ appears to have different pharmacokinetics and to be much less toxic than test QDs. While the distribution of CdTe-QDs observed in this study aligned with many previous studies, the toxic effects of CdTe-QDs in mice observed here disagreed with several studies. The disagreement could be due to several factors including doses, surface coating of QDs, and test animal models. This suggests that the toxicity of QDs in animal need to be assessed case by case basis before making conclusions on the impacts on human health. 


\section{Chapter: In Vivo Hepatotoxicity of Cadmium Telluride Quantum Dot Nanoparticles - Mitochondrial Effects As a Mechanism}

This chapter is based upon the following manuscript:

Kathy C. Nguyen, Yan (Mary) Zhang, Julie Todd, Douglas Parks, Michelle Lalande, Martha Navarro, William G. Willmore and Azam F. Tayabali. 2015. In Vivo Hepatotoxicity of Cadmium Telluride Quantum Dot Nanoparticles, Mitochondrial Effects as a Mechanism. To be submitted to Nanotoxicology journal.

For this manuscript, Kathy C. Nguyen designed and conducted these experiments and wrote the manuscript. Yan Zhang assisted with the animal necropsies. Julie Todd, Douglas Parks, Kelvin Kittle, Michelle Lalande, and Martha Navarro assisted with the animal exposures and necropsies. Drs. William Willmore and Azam Tayabali provided input into the manuscript. Dr. Azam Tayabali and Health Canada provided funding for the project. 


\subsection{Abstract}

The aim of this study was to investigate the detailed mechanisms of hepatotoxicity induced by CdTe-QDs in BALB/c mice after intravenous injection. The study examined oxidative stress, apoptosis in liver and effects on mitochondria as the potential mechanistic events that could elucidate the observed hepatotoxicity. Oxidative stress in the liver induced by CdTe-QDs was demonstrated by the depletion of total glutathione, increase in superoxide dismutase activity, and changes in gene expressions of several oxidative stress-related biomarkers. Furthermore, CdTe-QD treatments led to apoptosis in the liver via both intrinsic and extrinsic apoptotic pathways. Effects on mitochondria were evidenced by the enlargement and increase in the number of mitochondria in hepatocytes of treated mice. CdTe-QDs also induced changes in the levels and gene expressions of electron transport chain enzymes, depletion of ATP, and an increase in the mitochondrial biogenesis regulator, PGC-1 $\alpha$. The findings from this study suggest that CdTe-QDs induced toxicity in the liver and the underlying mechanisms of hepatotoxicity could have originated from mitochondrial effects which resulted in oxidative stress and apoptosis in the liver cells. This study provides insight into the biological effects of CdTe-QDs at the tissue level and the detailed mechanisms of their toxicity in animals. The study also provides important data for bridging the gap between in vitro and in vivo testing and for risk assessment of these NPs.

\subsection{Introduction}

Quantum dots (QDs) are rapidly emerging as an important class of nanoparticles (NPs) with wide applications in various fields ranging from medicine to energy. These 
engineered NPs, owing to their unique optical and electronic properties, have been widely used in microelectronics and biological research (De Wild et al., 2003), and have great potential in clinical diagnosis and therapeutic delivery (Chen et al., 2006; Gao et al., 2004; Scherer et al., 2002). As the use and production of QDs continue to grow, there has been increasing concern about the potential impacts of these NPs on human health and the environment. Besides, QD potential applications in medicine will lead to intentional exposures of these NPs to humans via different routes, mainly injection and oral administration. Thus, much information on toxicology of QDs is needed for assessment of health risks associated with exposure to these NPs.

Among various types of QDs, cadmium-based QDs attract the greatest attention due to their wide applications. Thus, in the past decade, the toxicity of cadmium-based QDs has been investigated in many in vitro and several in vivo studies (as reviewed by Hardman, 2006; Pelley et al., 2009; Yong et al., 2013). From the in vitro studies, it has been shown that cadmium-based QDs caused toxic effects in various cell lines. In addition, in in vitro it has been suggested that the toxicity of cadmium-based QDs is associated with the releases free cadmium ions $\left(\mathrm{Cd}^{2+}\right)$ and the generation of reactive oxygen species (ROS) from these NPs (Derfus et al., 2004; Chang et al., 2006; Samia et al., 2006; Maysinger and Lovric, 2007; Li et al., 2009; Chen et al., 2012; Nguyen et al., 2013). Several studies have also suggested that toxicity of CdTe-QDs could arise from several intrinsic properties such as size, chemical composition, reactivity of the inner core metals, and surface coating components (Hardman, 2006; Lovrić et al., 2005). While apparent toxicity of cadmium-based QDs was reported in many in vitro studies, toxicity of these NPs in vivo appeared to be inconclusive (Pelley et al., 2009; Yong et al., 2013). 
Thus, more information on toxicity of these NPs in animal models is required as well as the underlying mechanisms of their effects need to be thoroughly investigated.

Liver is the primary organ involved in the metabolism and detoxification of xenobiotics. Regardless of the routes that NPs enter the body to cause adverse health effects, NPs can reach the liver via the blood circulation and make it a potential target organ (Wang et al., 2007). To date, there are a limited number of studies on hepatotoxicity of NPs. There are a few in vitro and in vivo studies undertaken in the last couple of years demonstrating that NPs, including silver, nano-C60, copper, titanium dioxide and iron oxide magnetic NPs, are hepatotoxic to liver cell lines and in rodents. However, little is known of the mechanisms of hepatotoxicity caused by NPs used in these studies (Hussain et al., 2005; Sayes et al., 2005; Chen et al., 2006; Wang et al., 2007; Jain et al., 2008). In vivo studies on QD-induced hepatotoxicity have also been reported recently (Zhang et al., 2007; Lui et al., 2011, Lin et al., 2011, Tiwari et al., 2011). Unfortunately, assessment of QD acute toxicity to the liver in these studies was limited to only a few biological endpoints (such as cytotoxicity, glutathione peroxidase activity, and tissue histopathology) and therefore the investigators could only speculate on the mechanisms of QD-induced hepatotoxicity, without revealing the underlying mechanisms though more detailed empirical evidence.

In the previous studies (Chapter 2 and 3 of this research), we demonstrated that CdTe-QDs caused hepatotoxicity in vitro. Using HepG2 cell lines, we showed that CdTe-QDs caused cytotoxicity in a dose- and time-dependent manner, induced oxidative stress by increasing ROS production and interfering with antioxidant defenses in the cell model, leading to apoptosis (Nguyen et al., 2013b, Chapter 2). Furthermore, we showed 
the evidence of CdTe-QDs inducing mitochondrial damage in HepG2 cells, which appeared to be the mechanisms of CdTe-QD toxicity in this cell line (Nguyen et al., 2015, Chapter 3). In this present study, using BALB/c mice as an animal model and multiple toxicological endpoints, we tested the same hypothesis that CdTe-QDs are hepatotoxins in the mice and hepatotoxicity induced by these NPs is caused by mitochondrial damage in the hepatocytes which lead to oxidative stress and apoptosis in the liver of the animal model. The findings from this present in vivo study will provide important information for bridging the gap between in vitro and in vivo testing and for risk assessment of these NPs.

\subsection{Materials and Methods}

\subsubsection{Materials}

CdTe-QDs were purchased from Nano Impex Canada (Mississauga, ON). CdTeQDs were described by the manufacturer as mercaptopropionic acid (MPA) capped CdTe-QDs with a size of $5 \mathrm{~nm}$ and a spectral emission of $540 \mathrm{~nm}$. The stock of CdTeQDs was provided at a concentration of $5 \mathrm{mg} / \mathrm{ml}$ in water containing $20 \%(\mathrm{w} / \mathrm{w})$ of cadmium. Cadmium chloride $\left(\mathrm{CdCl}_{2}\right)$, obtained from Sigma-Aldrich (St. Louis, $\mathrm{MO}$ ). Glutaraldehyde, osmium tetroxide, and sodium cacodylate were purchased from Electron Microscopy Science (Hatfield, PA).

\subsubsection{Characterization of CdTe-QDs}

Characterization of CdTe-QDs used in this study was performed and described in Chapter 4 of this thesis. 


\subsubsection{Animal treatments and sample collection}

Animal treatments and sample collection were performed as described in the previous chapter (Chapter 4) of this thesis.

\subsubsection{Reduced GSH assay}

The cellular reduced GSH concentration was assayed using a glutathione colorimetric assay kit (Oxford Biomedical Research, Oxford, MI) according to the manufacturer's protocol.

\subsubsection{SOD assay}

Liver tissue samples were homogenized in cold $20 \mathrm{mM}$ 4-2-hydroxyethyl)-1piperazineethanesulfonic (HEPES) buffer ( $\mathrm{pH}$ 7.2) containing $1 \mathrm{mM}$ ethylene glycol tetraacetic acid (EGTA), $210 \mathrm{mM}$ mannitol, and $70 \mathrm{mM}$ sucrose, and centrifuged at 1,500 $\mathrm{x} g$ for $5 \mathrm{~min}$ at $4^{\circ} \mathrm{C}$. The supernatants were collected and centrifuged at $10,000 \mathrm{xg}$ for $15 \mathrm{~min}$ at $4^{\circ} \mathrm{C}$ to yield cytosolic SOD samples. The pellets were homogenized in cold HEPES buffer to yield mitochondrial SOD samples. SOD samples were assayed using a SOD colorimetric enzyme assay kit from Cayman Chemical (Ann Arbor, MI) according to the manufacturer's protocol.

\subsubsection{CAT assay}

CAT activity was measured using a catalase assay kit from Cayman Chemical (Ann Arbor, MI). In brief, liver tissue samples were homogenized in a cold buffer containing $50 \mathrm{mM}$ potassium phosphate $(\mathrm{pH} 7.0)$ and $1 \mathrm{mM}$ ethylenediaminetetraacetic 
acid (EDTA). The supernatants were collected after centrifugation at 10,000 x $\mathrm{g}$ for 15 min at $4^{\circ} \mathrm{C}$. To each well containing $20 \mu \mathrm{l}$ of standard, control, or sample, $100 \mu \mathrm{l}$ of assay buffer (100 mM potassium phosphate, $\mathrm{pH}$ 7.0, containing $1 \mathrm{mM}$ EDTA) and $30 \mu 1$ of methanol were added. $\mathrm{H}_{2} \mathrm{O}_{2}(20 \mu \mathrm{l})$ was added to each well. After the plate was incubated for $20 \mathrm{~min}$ at RT, $30 \mu \mathrm{l}$ of potassium hydroxide followed by $30 \mu 1$ of purpald was added to each well. The plate was then incubated for $10 \mathrm{~min}$ at RT. Finally, $10 \mu 1$ of potassium periodate was added to each well and the plate was incubated at RT for $5 \mathrm{~min}$ before measurement with a spectrophotometer at $540 \mathrm{~nm}$.

\subsubsection{GST activity assays}

GST activity was measured using GST enzymatic kit from Arbor Assays (Ann Arbor, MI) according to the manufacturer's instruction. Briefly, tissue samples were homogenized in the assay buffer and centrifuged at $1,500 \mathrm{xg}$ for $5 \mathrm{~min}$, at $4^{\circ} \mathrm{C}$ to obtain supernatant samples. Each sample or standard $(50 \mu 1)$ was added to the assigned well followed by $25 \mu 1$ of detection buffer and $25 \mu 1$ of GSH. The plate was incubated for 30 min at RT and then measured on a spectrofluorimeter with excitation at $390 \mathrm{~nm}$ and emission at $450 \mathrm{~nm}$.

\subsubsection{Caspase-3 activity and cleaved PARP assay}

Activated caspase-3 and cleaved PARP levels were measured using bead plex assay kits from EMD Millipore Corporation (Billerica, MA) according to the manufacturer's instructions. Briefly, tissue samples were homogenized in the provided lysis buffer and centrifuged at $12,000 \mathrm{rpm}$ for $20 \mathrm{~min}$ at $4^{\circ} \mathrm{C}$. The supernatants were 
collected, measured for protein concentration and diluted in assay buffer. Sample $(25 \mu 1)$ was added to the corresponding well in a 96-well filter plate containing $25 \mu 1$ of anticaspase-3 or PARP conjugated beads. The plate was incubated at $4^{\circ} \mathrm{C}$ overnight. After washing, $25 \mu 1$ of detection antibody was added to each well and the plate was incubated for $1 \mathrm{~h}$ at RT. Detection antibody was removed by vacuum filtration and $25 \mu 1$ of prediluted streptavidin-conjugated phycoerythrin was added to each well. The plate was incubated for 15 min at RT on a shaker. After vacuum filtration, $120 \mu$ of assay buffer was added to each well. The plate was shaken for $1 \mathrm{~min}$ and analyzed with the Bio-Plex 100 Array System (Bio-Rad, Hercules, CA).

\subsubsection{TEM of liver tissue}

Liver tissues were fixed with $2.5 \%$ glutaraldehyde and $4 \%$ paraformaldehyde in PBS. Fixed tissues were then submerged in $1 \%$ osmium tetroxide in $100 \mathrm{mM}$ cacodylate buffer, $\mathrm{pH} 7.4$ for $1 \mathrm{~h}$ at $4^{\circ} \mathrm{C}$, gradually dehydrated in ethanol, infiltrated with epoxy resin, and embedded onto resin-filled Beem ${ }^{\circledR}$ capsule molds. The blocks were then ultrathin-sectioned and stained first with uranyl acetate and then with lead citrate, and analysed with a Joel 1230 transmission electron microscope.

\subsubsection{Mitochondrial complex quantification assay}

The mitochondrial complex levels were measured using a BeadPlex ${ }^{\mathrm{TM}}$ mitochondrial oxidative phosphorylation kit from EMD (Gibbstown, NJ), according to the manufacturer's instructions. Briefly, tissue samples were rinsed with ice-cold PBS and then homogenized in supplied lysis buffer containing protease inhibitors. The cell 
lysates were centrifuged at $12,000 \mathrm{rpm}$ for $20 \mathrm{~min}$ at $4^{\circ} \mathrm{C}$ and the supernatants were collected and diluted in assay buffer. Each standard or sample (50 $\mu$ l) was added to the corresponding well in a filter 96 -well plate containing $25 \mu 1$ of anti-complex conjugated beads and $25 \mu \mathrm{l}$ of detection antibody. The plate was incubated for $3 \mathrm{~h}$ on a shaker at RT then washed three times with wash buffer before $50 \mu 1 /$ well of pre-diluted streptavidinconjugated phycoerythrin was added. The plate was incubated for $30 \mathrm{~min}$ on a shaker at RT. After being washed, $120 \mu$ l of wash buffer was added to each well. The plate was shaken for 1 min and analyzed with the Bio-Plex 100 Array System (Bio-Rad, Hercules, CA).

\subsubsection{RNA isolation and gene expression analyses}

The total liver tissue for RNA extraction varied between 10 and $15 \mathrm{mg}$ from each animal. RNA was isolated and purified from 5 to 6 individual animals from each of the treatment and control groups using RNA isolation kit from Qiagen (Toronto, ON) according to the manufacturer's instruction. Total RNA concentration was measured using a NanoDrop 2000 spectrophotometer (Thermo Fisher Scientific Inc., Wilmington, DE, USA), and RNA quality and integrity were assessed using an Agilent 2100 Bioanalyzer (Agilent Technologies, Inc., Mississauga, ON, Canada) according to the

manufacturer's instruction. All samples showed RNA integrity numbers of 7 and above, indicating high quality RNA, and were used to conduct qPCR experiments.

Gene expression analyses were performed using RT PCR array kits from Qiagen (Toronto, ON). Approximately $750 \mathrm{ng}$ of the total RNA ( $\mathrm{n}=3$ per group) from each of the experimental and control groups of mice was reverse transcribed using an $\mathrm{RT}^{2}$ first 
strand cDNA synthesis kit (Qiagen, Toronto, ON). qPCRs were conducted using $\mathrm{RT}^{2}$ SYBR Green qPCR Master Mix in a CFX96 Real-Time System (BioRad Laboratories, Mississauga, ON, Canada) according to the manufacturer's instructions. Threshold cycle (Ct) values were normalized using Hprt, Hsp90ab1, and Gapdh as internal control genes, and relative expression of the differentially expressed genes was determined using online PCR array data analysis software (Qiagen, Toronto, ON). Changes in gene expression were graphically presented as a fold increase or decrease, compared to that of untreated aniamals.

\subsubsection{ATP level detection}

ATP quantification was carried out using ATP colorimetric assay kit from Biovision (Mountain View, CA). Tissue samples were homogenized in ATP assay buffer. The samples were centrifuged at $15,000 \mathrm{~g}$ for $2 \mathrm{~min}$ at $4{ }^{\circ} \mathrm{C}$ and the supernatants were collected for the assay. Samples were diluted with ATP buffer and added to the corresponding wells on a microplate. CdTe-QDs alone were added to separate wells to test for the interference of the NPs with the assay system. To each well, $44 \mu 1$ of ATP buffer, $2 \mu 1$ of ATP probe, $2 \mu 1$ of ATP converter, and $2 \mu 1$ of developer solution were added. The plate was incubated for $30 \mathrm{~min}$ at RT and absorbance was measured at 570 $\mathrm{nm}$ in a microplate reader.

\subsubsection{PGC-1 $\alpha$ level detection}

PGC-1 $\alpha$ level was measured using PGC-1 $\alpha$ ELISA assay kit from BioSource (San Diego, CA). In brief, liver tissue samples were homogenized with ice-cold PBS and 
centrifuged at $5,000 \mathrm{~g}$ for $5 \mathrm{~min}$ at $4^{\circ} \mathrm{C}$. Supernatant was collected and assayed immediately. Each of the samples or standards $(100 \mu \mathrm{l})$ was added to the assigned well in the provided $96-$ well plate and incubated for $2 \mathrm{~h}$ at $37^{\circ} \mathrm{C}$. CdTe-QDs alone were added to separate wells to test for the interference of the NPs with the assay system. Biotinantibody $(100 \mu \mathrm{l})$ was then added to each well and the plate was incubated for $1 \mathrm{~h}$ at $37^{\circ} \mathrm{C}$. After three washes, $100 \mu \mathrm{l}$ of avidin-HRP was added to each well. After $1 \mathrm{~h}$ incubation and washes, $90 \mu 1$ of 3, 3', 5, 5' - tetramethylbenzidine (TMB) was added to each well followed by 15 min incubation. Stop solution $(50 \mu 1)$ was then added to each well and the absorbance was measured at $450 \mathrm{~nm}$ in a microplate reader.

\subsubsection{Statistical analysis}

Results were compared by one-way analysis of variance (ANOVA) followed by Dunnett's test for comparison of treatment groups to the negative control group and Tukey's test for pairwise comparison of $6 \mathrm{mg} / \mathrm{kg}$ bw CdTe-QD and $\mathrm{CdCl}_{2}$ treated groups. All data was expressed as mean \pm standard deviation. A value of $p<0.05$ was considered as statistically significant.

\subsection{Results}

\subsubsection{Characterization of CdTe-QDs}

The characterization of CdTe-QDs used in this study was performed and described in Chapter 4 of this thesis.

\subsubsection{Induction of liver oxidative stress}


Several oxidative stress biomarkers were measured in the liver to examine the induction of oxidative stress by CdTe-QDs in hepatocytes. Starting at a concentration of $2 \mathrm{mg} / \mathrm{kg}$ bw, CdTe-QD treatments resulted in a depletion of total glutathione (tGSH) and an increase in total superoxide dismutase (SOD) activity (Figure 5.1.). Exposure to $\mathrm{CdCl}_{2}$ at an equivalent cadmium concentration to $6 \mathrm{mg} / \mathrm{kg}$ bw CdTe-QDs also lead to changes in the above biomarkers. At $5 \mathrm{mg} / \mathrm{kg}$ bw of CdTe-QDs, the depletion of tGSH and elevation of SOD activity in treated mice occurred at $2 \mathrm{~h}$ and retained at similar levels up to $1 \mathrm{w}$. Both CdTe-QDs (starting at $6 \mathrm{mg} / \mathrm{kg}$ bw) and $\mathrm{CdCl}_{2}$ also caused decreases in the activities of antioxidant enzymes, glutathione-S-transferase (GST) and catalase (Figure 5.2.). Oxidative stress in liver hepatocytes was also examined by measuring the gene expression of certain antioxidant biomarkers as detected using PCR array. CdTe-QDs and $\mathrm{CdCl}_{2}$ appeared to up-regulate gene expression of oxidative stress responsive genes including heme oxygenase I (gene ID: Hmox I, Figure 5.3.) and neutrophil cytosol factor 1 and 2 (Gene ID: Ncf1 and Ncf2, respectively, Figure 5.3.). CdTe-QDs and $\mathrm{CdCl}_{2}$ also down-regulated the expression of antioxidant genes such as catalase (gene ID: CAT, Fig. 5.3.) and GST (gene ID: Gstk1 and Gstp1, Figure 5.3.). In addition, while CdTe-QDs appeared to have no or little effects on the gene expression of glutathione peroxidase (gene ID: Gpx1-7, Figure 5.3.) and peroxiredoxin (gene ID: Prxd 3, 4 and 6, Figure 5.3.), $\mathrm{CdCl}_{2}$ induced significant changes in the expression of these genes (Figure 5.3.). 
A

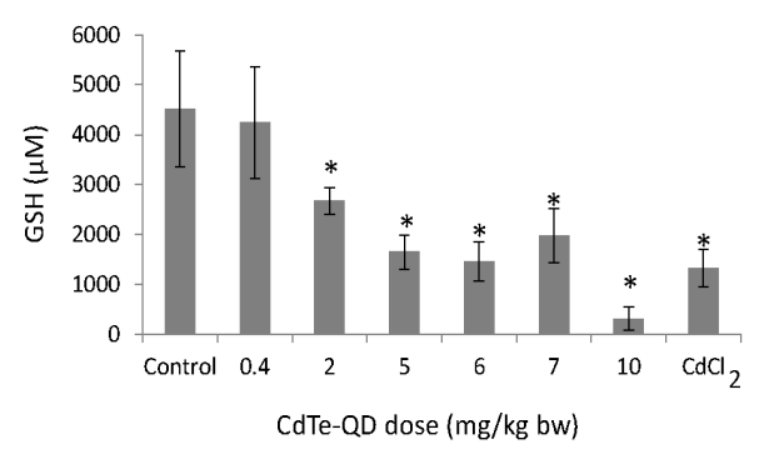

C

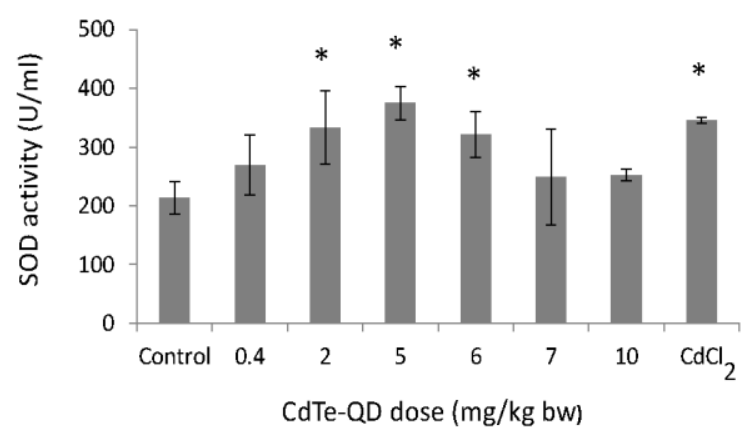

B

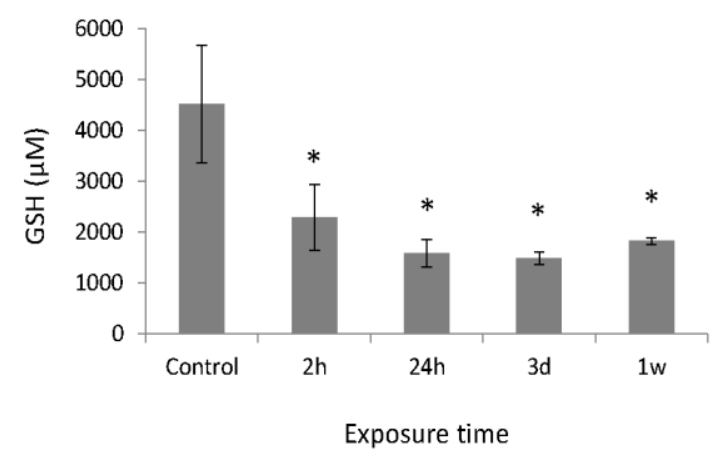

D

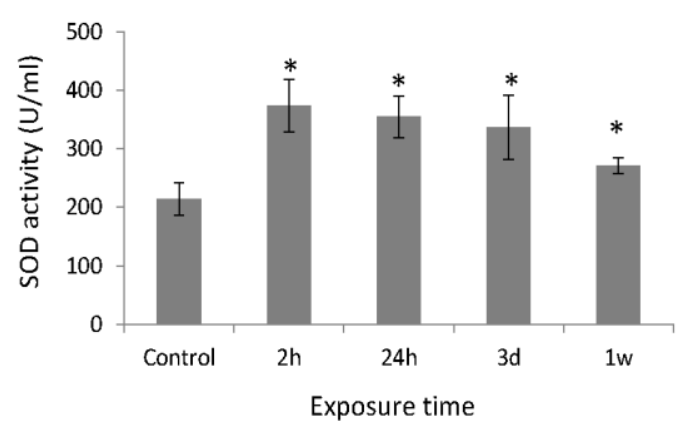

Figure 5.1. Effects of $\mathrm{CdTe}-\mathrm{QDs}$ and $\mathrm{CdCl}_{2}$ on biomarkers of oxidative stress. (A) Total GSH level in the liver at $24 \mathrm{~h}$ post-exposure, (B) total GSH level in the liver at different post-exposure sampling times, $(\mathbf{C})$ total SOD activities in the liver at $24 \mathrm{~h}$, and (D) total SOD activity in the liver at different post-exposure sampling times. (A) and (C) Mice were treated with different concentrations of CdTe-QDs and $1.95 \mathrm{mg} / \mathrm{kg}$ bw $\mathrm{CdCl}_{2}$ which contains an equivalent cadmium concentration to $6 \mathrm{mg} / \mathrm{kg}$ bw CdTe-QDs for $24 \mathrm{~h}$. (B) and (D) Mice were treated with $5 \mathrm{mg} / \mathrm{kg}$ bw CdTe-QDs and biomarkers were measured at different post-exposure sampling times. The asterisks $(*)$ indicate statistically significant differences compared to the control $(p<0.05)$. 
A

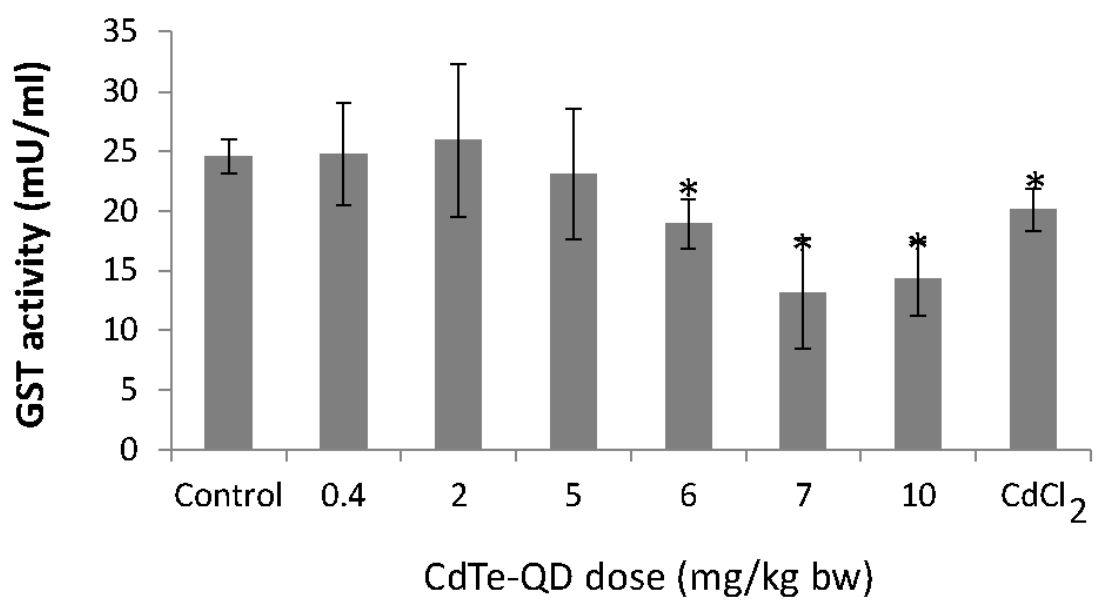

B

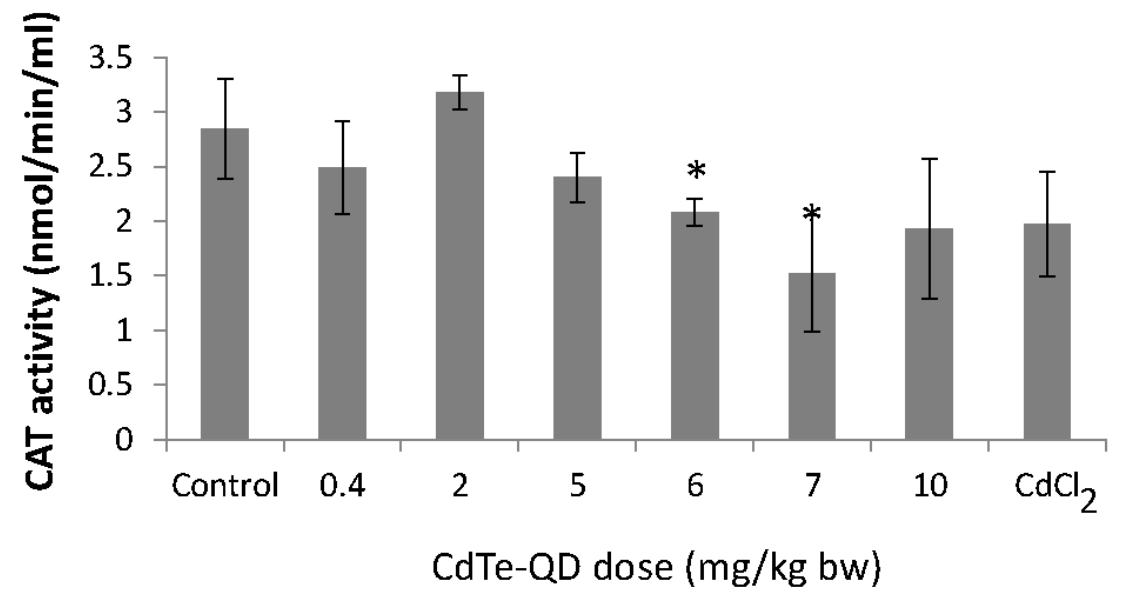

Figure 5.2. Effects of $\mathrm{CdTe}-\mathrm{QDs}$ and $\mathrm{CdCl}_{2}$ on antioxidant enzymes. (A) GST activity in the liver (B) CAT activity in the liver. Mice were treated with different concentrations of CdTe-QDs and $1.95 \mathrm{mg} / \mathrm{kg}$ bw $\mathrm{CdCl}_{2}$, which contains an equivalent cadmium concentration to $6 \mathrm{mg} / \mathrm{kg}$ bw CdTe-QDs, for $24 \mathrm{~h}$. The asterisks (*) indicates statistically significant differences compared to the control $(\mathrm{p}<0.05)$. 


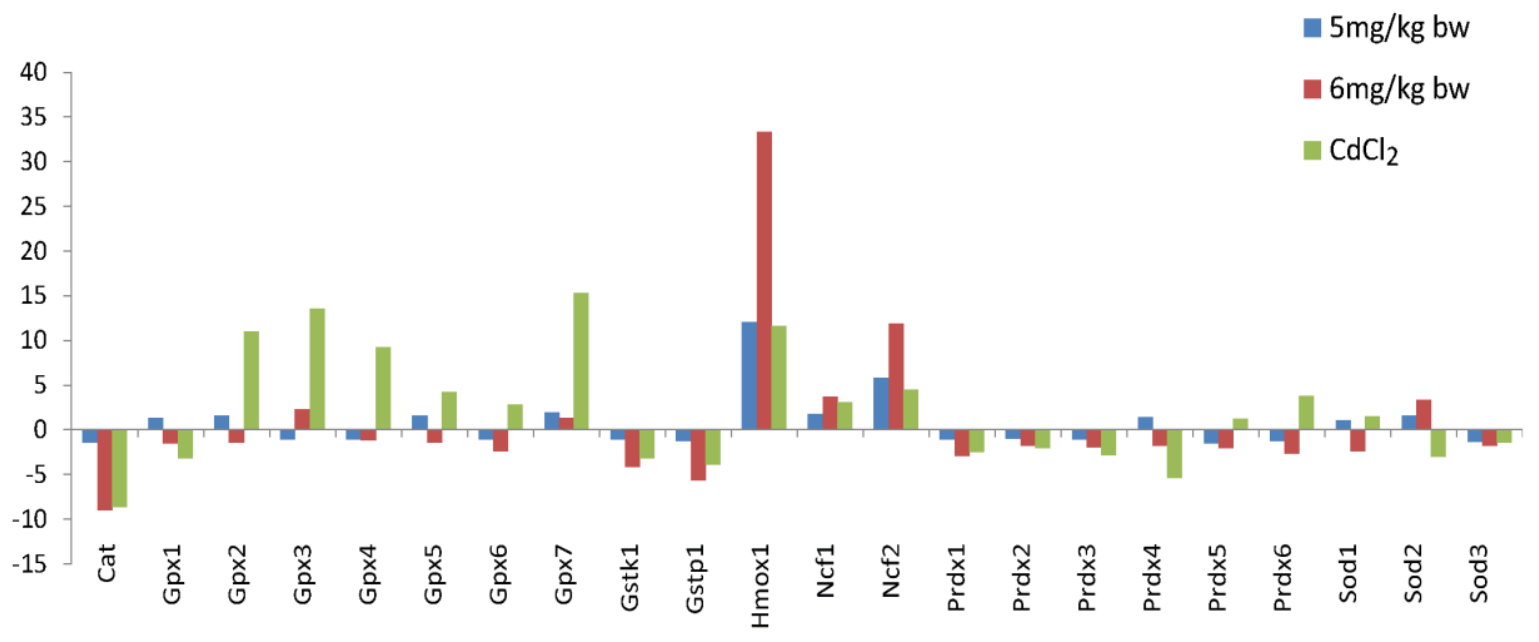

Figure 5.3. Alteration of oxidative stress gene expression caused by CdTe-QDs and $\mathrm{CdCl}_{2}$. Mice were treated with different concentrations of CdTe-QDs and $1.95 \mathrm{mg} / \mathrm{kg}$ bw $\mathrm{CdCl}_{2}$, which contains an equivalent cadmium concentration to $6 \mathrm{mg} / \mathrm{kg}$ bw CdTe-QDs, for $24 \mathrm{~h}$. Cat $=$ catalase, $\mathrm{Gpx}=$ glutathione peroxidase, Gstk $=$ glutathione $\mathrm{S}$-transferase kapa, Gstp = glutathione S-transferase pi, Hmox $=$ heme oxygenase, Ncf $=$ neutrophil cytosolic factor, Prdx $=$ peroxiredoxin, Sod $=$ superoxide dismutase. $(+)=$ mRNA up regulation, $(-)=$ mRNA down-regulation.

\subsubsection{Induction of apoptosis in the liver}

Apoptosis induced by CdTe-QDs (at 6-10 mg/kg bw) was evidenced by significant elevations of active caspase- 3 and cleaved PARP, which are the hallmarks of apoptosis (Figure 5.4.). Treatment with $\mathrm{CdCl}_{2}$, at an equivalent of cadmium concentration to $6 \mathrm{mg} / \mathrm{kg}$ bw CdTe-QDs, did not cause significant changes in active caspase-3 and cleaved PARP (Figure 5.4.). Further examination of apoptosis in the liver using RT-PCR array showed significant up-regulation of numerous apoptosis-related genes from CdTe-QD treatments (Figure 5.5.). In addition, CdTe-QDs altered the gene 
expression of key biomarkers of the intrinsic apoptosis (Gene ID: Bid, Bax, and Bcl2) and of the extrinsic apoptosis (Gene ID: Casp8, Fas, and Fasl) (Figure 5.5.). $\mathrm{CdCl}_{2}$ also induced changes in the expression of several apoptosis-related genes. However, the level of effects from $\mathrm{CdCl}_{2}$ was much less compared to that of $6 \mathrm{mg} / \mathrm{kg}$ bw CdTe-QDs (Figure 5.5.).

A

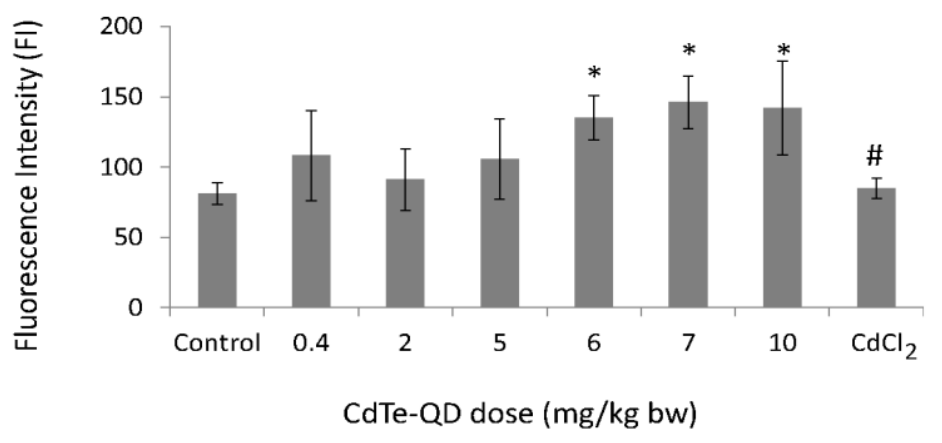

B

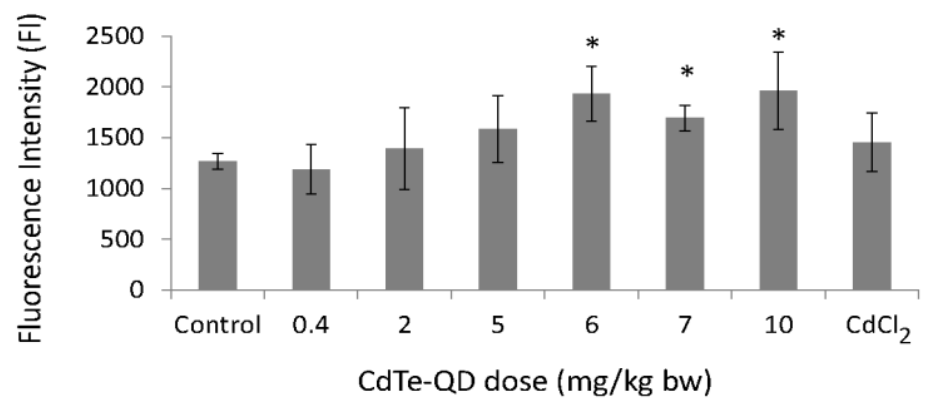

Figure 5.4. Effects of CdTe-QDs and $\mathrm{CdCl}_{2}$ on the apoptosis biomarkers. (A) Active caspase-3 level and (B) cleaved PARP level. Data were represented by fluorescence intensities (FI). Mice were treated with different concentrations of CdTe-QDs and 1.95 $\mathrm{mg} / \mathrm{kg}$ bw $\mathrm{CdCl}_{2}$, which contains an equivalent cadmium concentration to $6 \mathrm{mg} / \mathrm{kg}$ bw CdTe-QDs, for $24 \mathrm{~h}$. The asterisks $\left(^{*}\right)$ indicates statistically significant differences compared to the control $(\mathrm{p}<0.05)$. The number $(\#)$ sign indicates statistically significant differences of $\mathrm{CdCl}_{2}$ compared to the CdTe-QD-treated group ( $\mathrm{p}<0.05$ ). 


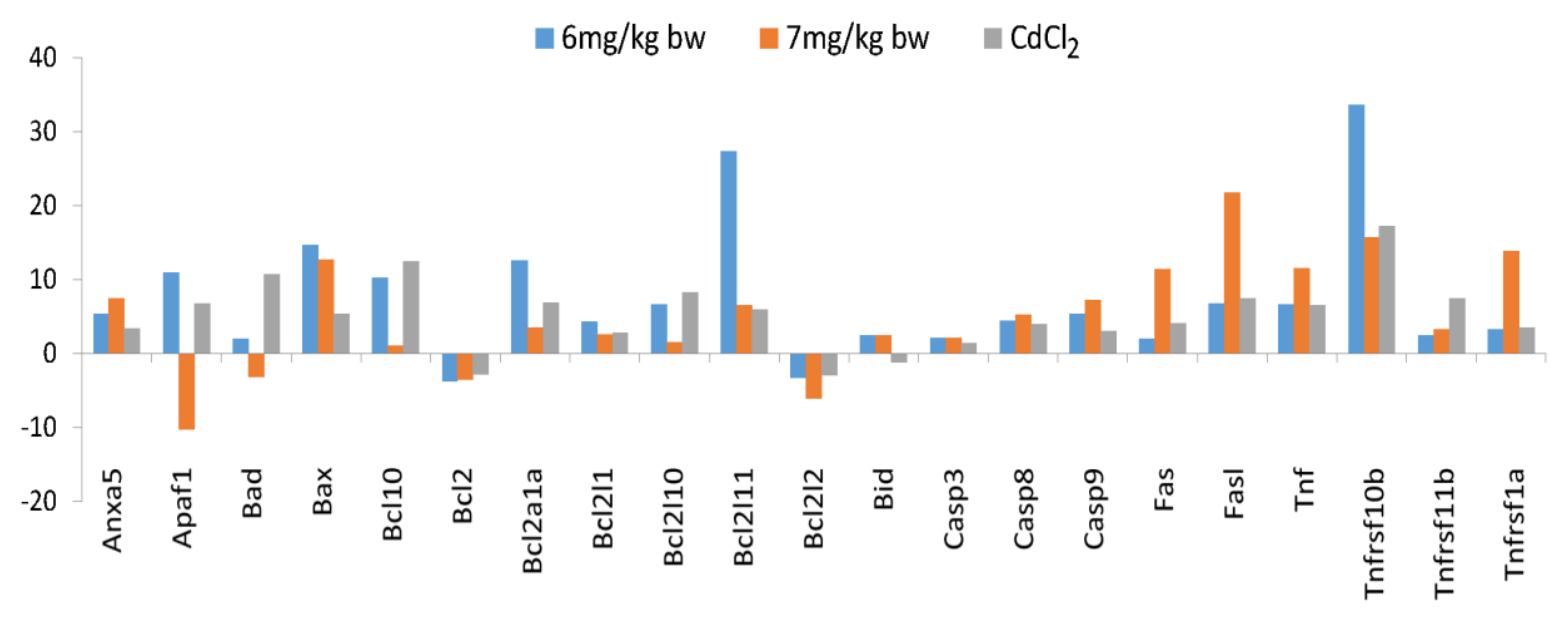

Figure 5.5. Alteration of apoptotic gene expression caused by $\mathrm{CdTe}-\mathrm{QDs}$ and $\mathrm{CdCl}_{2}$. Mice were treated with different concentrations of CdTe-QDs and $1.95 \mathrm{mg} / \mathrm{kg} \mathrm{bw} \mathrm{CdCl}_{2}$, which contains an equivalent cadmium concentration to $6 \mathrm{mg} / \mathrm{kg}$ bw CdTe-QDs for $24 \mathrm{~h}$. $\operatorname{Anxa}=, \operatorname{Apaf}=, \operatorname{Bad}=, \mathrm{Bax}=, \mathrm{Bcl}=, \mathrm{Bid}=, \mathrm{Casp}=, \mathrm{Fas}=, \mathrm{Fasl}=$, Tnf $=$, Tnfrsf $=.(+)=$ mRNA Up regulation, $(-)=$ mRNA Down-regulation .

\subsubsection{Liver mitochondrial morphology}

The integrity of mitochondria from liver hepatocytes was examined using TEM. Enlargement of mitochondria was observed at $5 \mathrm{mg} / \mathrm{kg}$ bw or higher (Figure 5.6.). An increase of mitochondrial number was notable at $6 \mathrm{mg} / \mathrm{kg}$ bw or higher. At the highest dose, $10 \mathrm{mg} / \mathrm{kg} \mathrm{bw}$, the formation vacuoles was observed and mitochondria were surrounded by air spaces (Figure 5.6.). Alteration of mitochondrial morphology and number was not observed in hepatocytes from mice treated with $\mathrm{CdCl}_{2}$ (Figure 5.6.). 

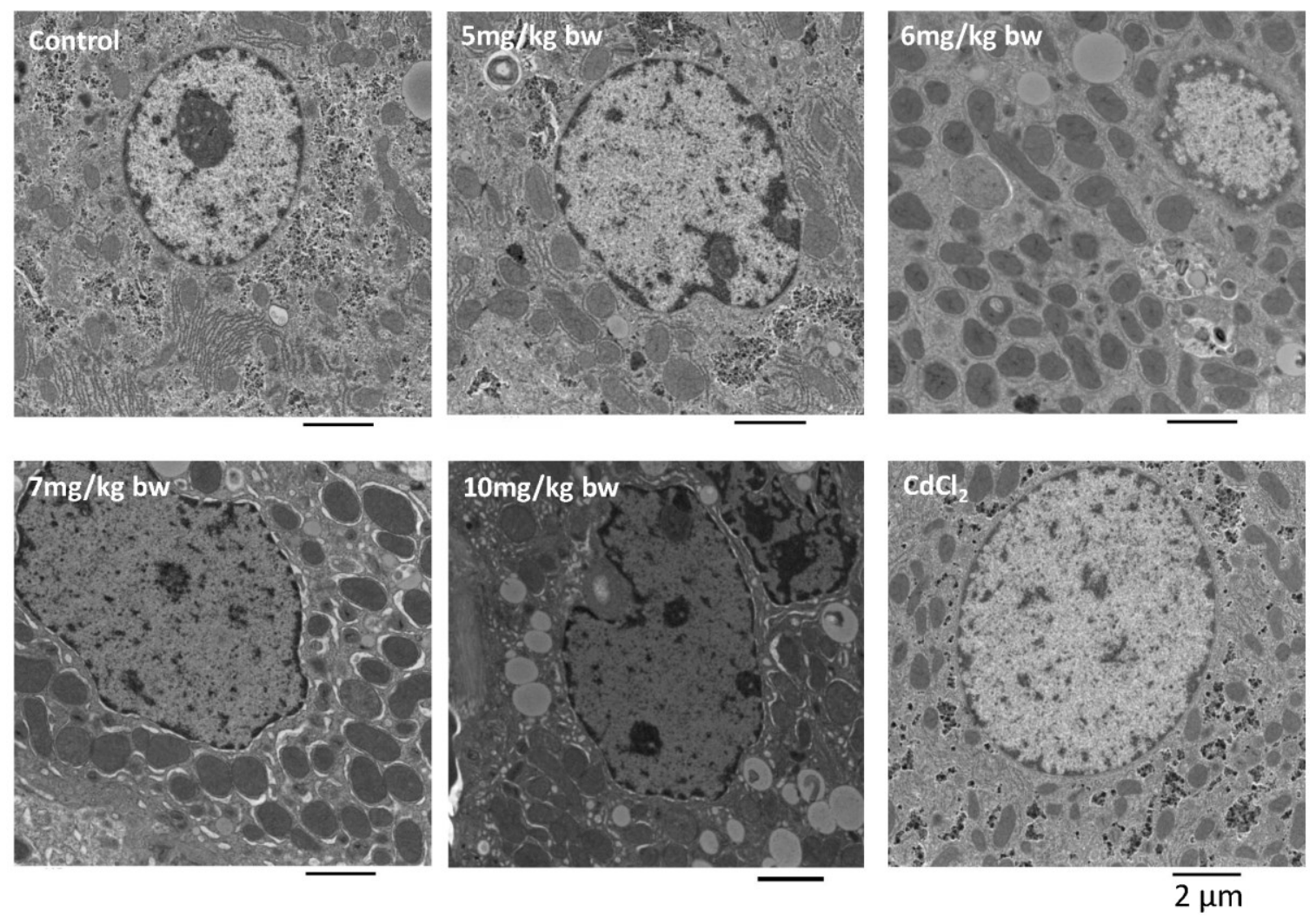

Figure 5.6. Changes in liver hepatocyte mitochondrial morphology, structure, and number induced by CdTe-QDs as examined by TEM. Mice were treated with different concentrations of CdTe-QDs and $1.95 \mathrm{mg} / \mathrm{kg}$ bw $\mathrm{CdCl}_{2}$, which contains an equivalent cadmium concentration to $6 \mathrm{mg} / \mathrm{kg}$ bw CdTe-QDs, for $24 \mathrm{~h}$.

\subsubsection{Effects on liver mitochondrial complexes}

The effects of CdTe-QDs on the mitochondrial ETC complexes were examined by measuring the levels of these complexes in the liver tissues. Only complexes I, III and $\mathrm{V}$ were measured in this study as there was no quantification assay kits for mouse complex II and complex IV available. CdTe-QD exposure decreased complex I and III levels while increased complex V level (Figure 5.7.A). Similar to CdTe-QDs, $\mathrm{CdCl}_{2}$ also 
increased complex V level. However, the compound did not alter complex I and III levels (Figure 5.7.A). Gene expression analyses showed that CdTe-QDs down-regulated the gene expression of sub-units of complexes I, II, III and IV, but up-regulated that of complex $\mathrm{V}$ (Figure 5.7.B). For $\mathrm{CdCl}_{2}$ treatment, up-regulation of complex $\mathrm{V}$ gene expression was also observed. However, $\mathrm{CdCl}_{2}$ did not induce significant effects on the gene expression of complexes I, II and III (Figure 5.7.B).

A
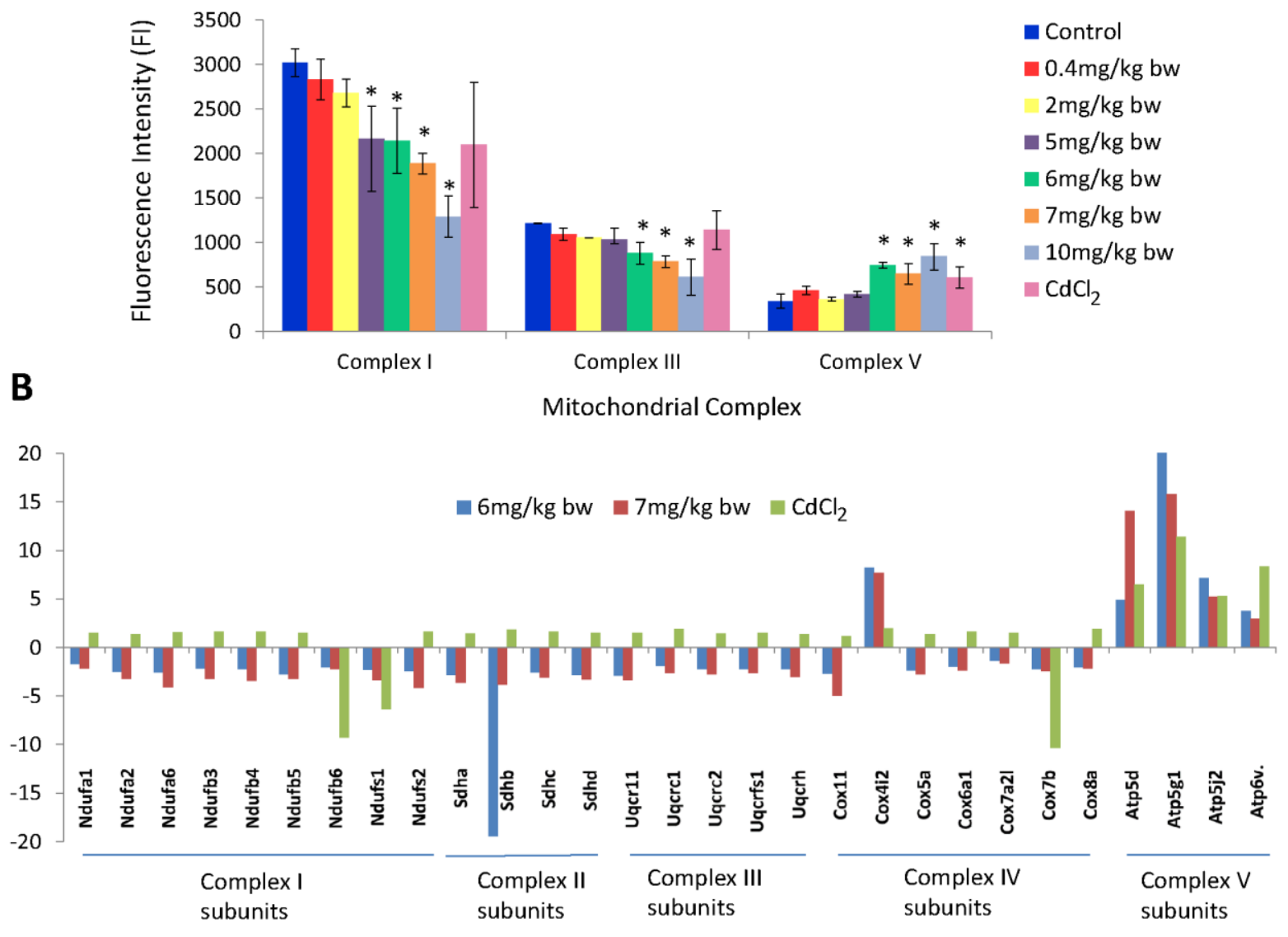

Figure 5.7. Effects of $\mathrm{CdTe}-\mathrm{QDs}$ and $\mathrm{CdCl}_{2}$ on the mitochondrial ETC complexes. (A) Alteration of the ETC complexes at the protein level. The asterisks $(*)$ indicates statistically significant differences compared to the control ( $p<0.05)$. (B) Changes in 
ETC complex gene expression. Mice were treated with different concentrations of CdTeQDs and $1.95 \mathrm{mg} / \mathrm{kg}$ bw $\mathrm{CdCl}_{2}$, which contains an equivalent cadmium concentration to $6 \mathrm{mg} / \mathrm{kg} / \mathrm{ml} \mathrm{CdTe-QDs}$, for $24 \mathrm{~h}$. Ndufa = NADH dehydrogenase Complex I subunit; Sdhd = Succinate dehydrogenase, Complex II, subunit D; Uqcr = Ubiquinol-cytochrome c reductase, Complex III, subunit; Cox $=$ Cytochrome c Oxidase, Complex IV, subunit; Atp = ATP synthase, complex V, subunit. $(+)=$ mRNA up regulation, $(-)=$ mRNA down-regulation.

\subsubsection{Effects on liver ATP content}

ATP content was determined in the liver to assess the effects of CdTe-QDs on ATP synthesis in treated mice. At high doses $(6-10 \mathrm{mg} / \mathrm{kg} \mathrm{bw})$, treatments of CdTe-QDs led to a decrease in ATP level in the liver (Figure 5.8.). By contrast, $\mathrm{CdCl}_{2}$ treatment did not cause changes in the ATP level (Figure 5.8.).

\subsubsection{Effects on liver mitochondrial biogenesis}

The effect of CdTe-QDs on mitochondrial biogenesis was examined by assessing the level of transcriptional factor PGC-1 $\alpha$, which acts as a regulator of mitochondrial number and function. At high doses, $5-7 \mathrm{mg} / \mathrm{kg} \mathrm{bw}, \mathrm{CdTe}-\mathrm{QDs}$ caused significantly increased PGC-1 $\alpha$ levels (Figure 5.9.A). At the highest dose (10 mg/kg bw), the level of PGC-1 $\alpha$ appeared comparable to that of the control (Figure 5.9.A). Contrary to CdTeQDs, $\mathrm{CdCl}_{2}$ did not cause a change in the PGC-1 $\alpha$ level (Figure 5.9.A). Treatment of 5 $\mathrm{mg} / \mathrm{kg}$ bw CdTe-QDs also significantly elevated PGC-1a at $3 \mathrm{~d}$, but the elevation was insignificant at $1 \mathrm{w}$ (Figure 5.9.B). 

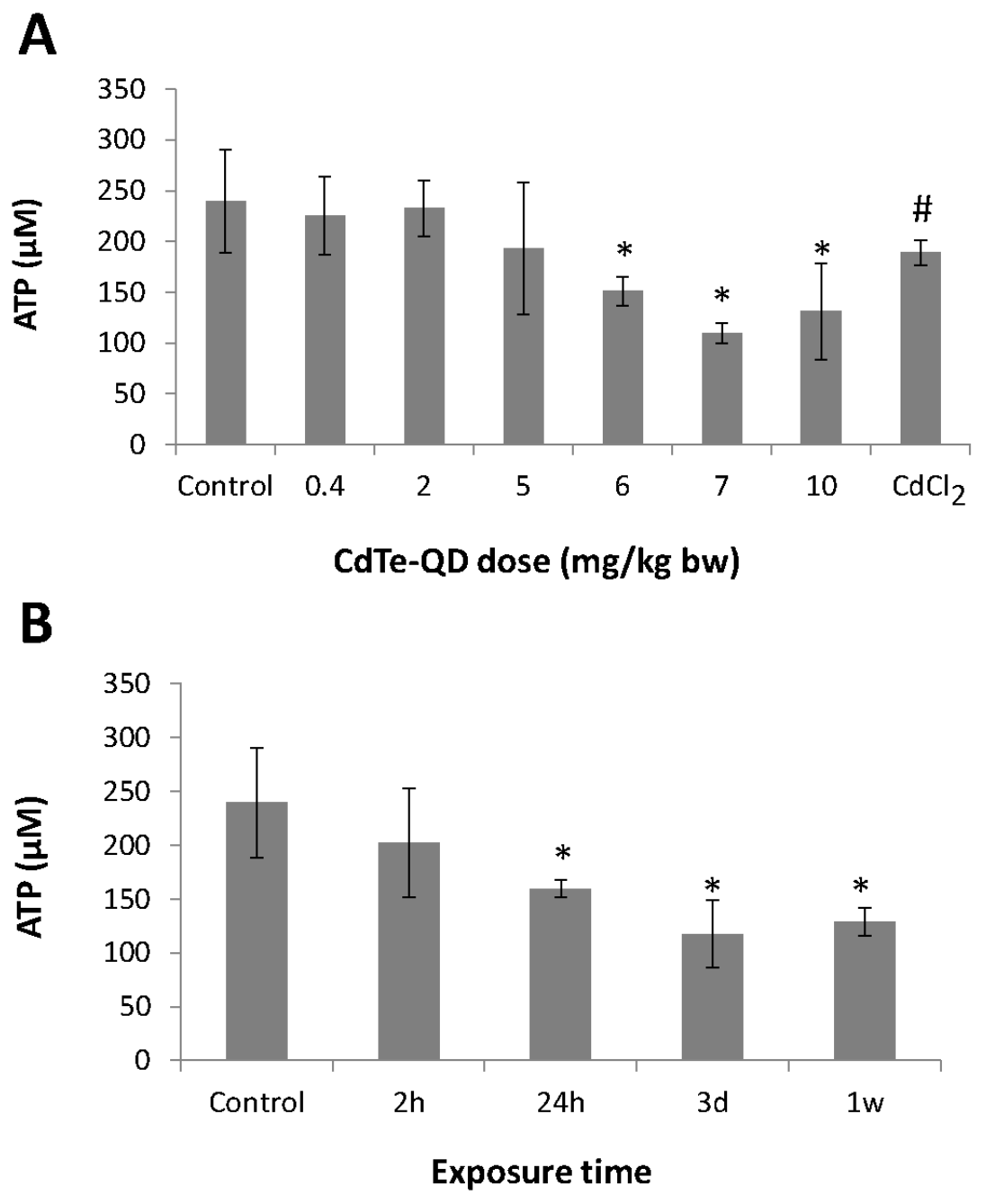

Figure 5.8. Effect of CdTe-QDs and $\mathrm{CdCl}_{2}$ on the ATP level. (A) Mice were treated with different concentrations of CdTe-QDs and $1.95 \mathrm{mg} / \mathrm{kg}$ bw $\mathrm{CdCl}_{2}$, which contains an equivalent cadmium concentration to $6 \mathrm{mg} / \mathrm{kg}$ bw CdTe-QDs for $24 \mathrm{~h}$. (B) Mice were treated with $5 \mathrm{mg} / \mathrm{kg}$ bw CdTe-QDs for different time points. The asterisks (*) indicates statistically significant differences compared to the control $(\mathrm{p}<0.05)$. The number $(\#)$ sign indicates statistically significant difference compared to the CdTe-QD-treated group $(\mathrm{p}<0.05)$ 


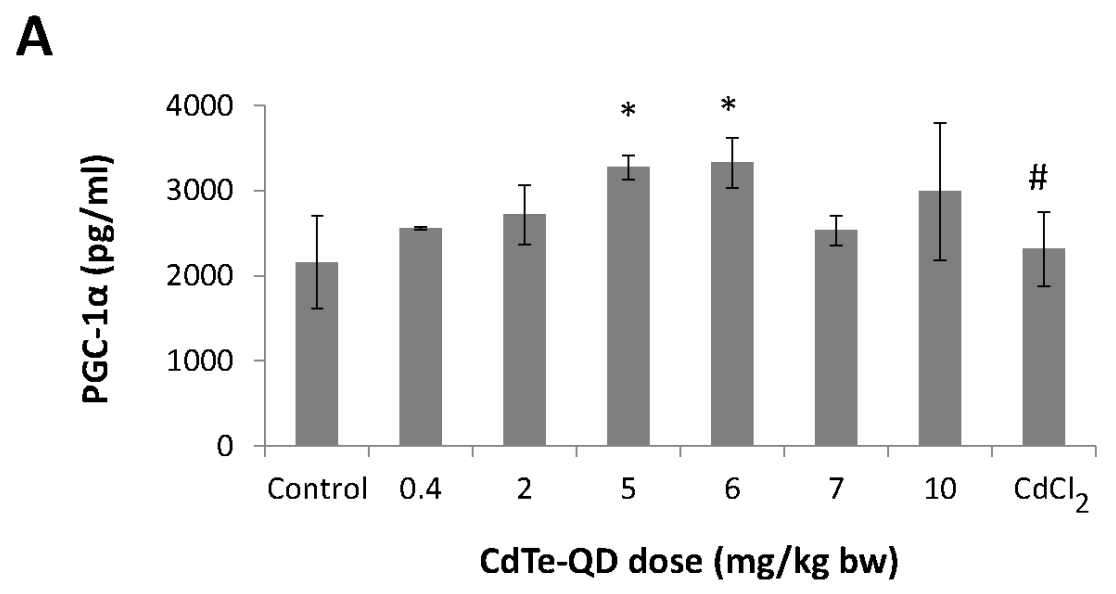

B

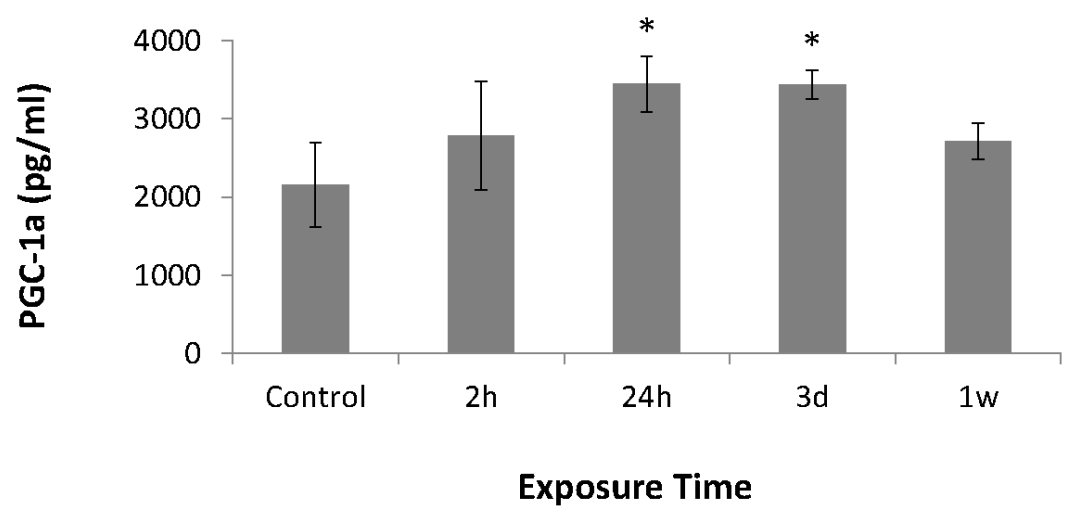

Figure 5.9. Effect of $\mathrm{CdTe}-\mathrm{QDs}$ and $\mathrm{CdCl}_{2}$ on mitochondrial biogenesis biomarker, PGC-1 $\alpha$ in the liver. (A) Mice were treated with different concentrations of CdTe-QDs and $1.95 \mathrm{mg} / \mathrm{kg}$ bw $\mathrm{CdCl}_{2}$, which contains an equivalent cadmium concentration to 6 $\mathrm{mg} / \mathrm{kg}$ bw CdTe-QDs for $24 \mathrm{~h}$. (B) Mice were treated with $5 \mathrm{mg} / \mathrm{kg}$ bw CdTe-QDs for different time points. The asterisks $(*)$ indicates statistically significant differences compared to the control $(\mathrm{p}<0.05)$. The number $(\#)$ sign indicates statistically significant difference compared to the CdTe-QD-treated group $(\mathrm{p}<0.05)$. 


\subsection{Discussion}

The increase in the production of QDs in the last few years, due to their widespread uses in different fields and to their potential applications in biomedical research as imaging and diagnostic agents, has led to the urgent need for hazard assessment of these NPs. Toxicity of cadmium-based QDs has been shown in various cells lines, but has been inconclusive in animal models (Hardman, 2006; Pelley et al., 2009; Yong et al., 2013). Although a few studies have reported the toxicity of various types of QDs in rodents, the little is known about the detailed mechanisms underlying these effects. In the previous studies (Chapter 2 and 3 of this thesis), we reported hepatotoxicity of CdTe-QDs in human hepatocytes HepG2 and discussed the underlying mechanisms of the in vitro toxicity of these NPs (Nguyen et al., 2013b and Nguyen et al., 2015). In this present study, which is an extension of the previous chapters, the hepatotoxicity of CdTe-QDs in BALB/c mice was demonstrated and the underlying mechanisms was investigated to elucidate the roles of mitochondria in observed effects in the liver of treated animals.

The induction of oxidative stress and apoptosis in the liver by CdTe-QDs were first studied by examining changes in several oxidative stress and apoptosis biomarkers. CdTe-QD treatments resulted in a depletion of total glutathione (tGSH) and an increase in total superoxide dismutase (SOD) activity, indicating oxidative stress occurred in treated mice. CdTe-QDs also caused decreases in the activities of antioxidant enzymes such as GST and catalase, suggesting that CdTe-QDs interfered with the anti-oxidant system in the liver of treated mice. $\mathrm{CdCl}_{2}$, at an equivalent cadmium concentration to one of the test CdTe-QD doses, was used for comparing the effects of cadmium in its 
ionic state with those of cadmium in CdTe-QDs. $\mathrm{CdCl}_{2}$ also induced similar effects on GSH, SOD, and GST, suggesting the roles of cadmium in CdTe-QD effects on the liver. Oxidative stress in the liver was also studied by measuring the expression of certain oxidative stress related genes. $\mathrm{CdTe}-\mathrm{QDs}$ and $\mathrm{CdCl}_{2}$ appeared to up-regulate gene expression of several oxidative responsive genes including Hmox I, Ncf-1 and Ncf-2, and down-regulated the gene expression of catalase and GST. These results correlated with the effects on the GSH level and antioxidant enzymes activities induced by CdTe-QDs and $\mathrm{CdCl}_{2}$ as previously discussed. These results support our previous in vitro study showing that CdTe-QDs caused oxidative stress in human hepatocyte HepG2 cells (Nguyen et al., 2013b). Our findings also agree with other groups' studies reporting oxidative stress induced by different types of QDs, including the same type that was used in this present study, in rodent models (Haque et al., 2011; Lin et al., 2011; Lui et al., 2011; Tiwari et al., 2011). Oxidative stress occurred at the treatment of $2 \mathrm{mg} / \mathrm{kg} b w$, which is a low dose in this treatment regime that did not cause any observable effects in other examined endpoints, suggesting that oxidative stress was an early event in CdTeQD toxicity. In this study, it is worthy to notice that while CdTe-QDs appeared to have no effects on the gene expression of glutathione peroxidase and peroxiredoxin, $\mathrm{CdCl}_{2}$ induced significant changes in these. These results suggested that the underlying mechanisms and pathways of oxidative stress induced by CdTe-QDs and $\mathrm{CdCl}_{2}$ might not be the same. The differential effects of CdTe-QDs and $\mathrm{CdCl}_{2}$ in oxidative stress induction revealed that cadmium is not the only factor that contributes to CdTe-QD toxicity. 
Apoptosis or programmed cell death is a ubiquitous process that regulates physiological growth control and regulation of tissue homeostasis (Fulda and Debatin, 2006). Apoptosis can be initiated by a variety of stimuli, and oxidative stress has been shown to be an important factor that triggers apoptosis (Carmody and Cotter, 2001). Apoptosis can be activated either via the death-receptor mediated extrinsic pathway or via the mitochondria-directed intrinsic pathway (Fulda and Debatin, 2006). The extrinsic pathway, triggered by ligands binding plasma membrane death receptors, leads to activation of initiator caspase 8 (Fas et al, 2006). On the other hand, the intrinsic pathway is mediated by pro- and anti-apoptotic Bcl-2 family proteins. This pathway leads to the release of cytochrome $\mathrm{c}$ from mitochondria and activation of the apoptosome complex, initiator caspase 9 and the effector caspases (Crompton, 2000). In this study, apoptosis induced by CdTe-QDs was evidenced by significant elevations of active caspase-3 and cleaved PARP, which are the hallmarks of apoptosis (Slee et al., 1999). Similarly, the RT-PCR array analyses showed significant up-regulations of numerous apoptosis-related genes. In addition, CdTe-QDs up-regulated the gene expression of key biomarkers of the intrinsic apoptosis including Bid, Bax, and Bcl2 and of the extrinsic apoptosis such as Casp8, Fas, and Fasl. The results confirmed the findings from our in vitro study, which showed that CdTe-QDs induced apoptosis in both extrinsic and intrinsic pathways (Nguyen et al., 2013b). Apoptosis induced by CdTe-QDs also confirmed the previous liver pathology and hepatocyte morphology results showing that high doses of CdTe-QDs caused apoptosis in hepatocytes as examined by H\&E staining (Chapter 4 of this thesis). Although $\mathrm{CdCl}_{2}$ appeared to induce alterations in the gene expression of several apoptotic markers, the compound did not cause changes in active 
caspase-3 and cleaved PARP at the protein level. This can be explained by the fact that the test concentration of $\mathrm{CdCl}_{2}$ used in this study was much lower than the toxic concentrations of this compound in animals that was reported in many previous studies (Dudley et al., 1984; Kotsonis et al., 1977; Kuester et al., 2002). The differential effects between $\mathrm{CdTe}-\mathrm{QDs}$ and $\mathrm{CdCl}_{2}$ at an equivalent concentration of cadmium also revealed that $\mathrm{CdTe}-\mathrm{QDs}$ are more toxic than $\mathrm{CdCl}_{2}$, and in addition to the effects from cadmium, the toxicity of these CdTe-QDs could also come from their other intrinsic physicochemical properties including small size and redox activity.

The mitochondrion is one of the most important organelles in the cell as it plays a critical role in cellular energy production which is achieved through oxidative phosphorylation involving the electron transport chain (ETC) (Galante et al., 1979). The proteins of the ETC, namely Complexes I, II, III, IV and V, are located in the inner membrane of the mitochondria. In oxidative phosphorylation, electrons are transferred along Complex I to IV from low reduction-oxidation (redox) potential to high redox potential, creating a proton gradient across the inner mitochondrial membrane, which is used by Complex V to drive the synthesis of ATP (Duchen, 2004). Mitochondrial damage has been suggested as mechanisms of apoptosis through one of the following processes: 1) disruption of electron transport, oxidative phosphorylation and ATP production, 2) release of proteins triggering activation of caspase family proteases and 3) alteration of cellular redox potential (Green and Reed, 1998). Thus, the oxidative stress and apoptosis observed in this study could be a results of the effects of CdTe-QDs on mitochondria. 
Mitochondria have been suggested as potential target organelles in NP toxicity (Nel et al., 2006; Xia et al., 2006). However, there is little known information on exactly how NPs interact with the mitochondria and affect theirs function. In this study, detailed examination on mitochondria in the liver showed that CdTe-QDs induced enlargement of mitochondria and caused increased mitochondria number in hepatocytes in treated mice as examined by TEM. The enlargement of mitochondria indicated effects on mitochondrial membrane and mitochondrial permeability transition (MPT) while the increase in mitochondrial number suggested stimulation of mitochondrial biogenesis. Since our previous study (Chapter 3) has shown the association of CdTe-QDs with mitochondria in HepG2 cells (Nguyen et al., 2015), the enlargement of mitochondria observed in this study could be a result of direct interaction of these NPs with mitochondrial membrane in the hepatocytes of the liver. Our study went further to assess the effect of CdTe-QDs on mitochondrial biogenesis by examining the level of transcriptional factor PGC-1 $\alpha$, which acts as a regulator of mitochondrial number and function and as an indicator of mitochondrial biogenesis (Knutti and Kralli, 2001). At high doses, CdTe-QDs caused significantly increased PGC-1 $\alpha$ levels. This result supported the increased mitochondrial number observed by TEM analysis and shows evidence for activation of mitochondrial biogenesis induced by CdTe-QDs in hepatocytes of treated mice. Several studies have shown that the activation of PGC-1 $\alpha$ could be triggered by ATP depletion as a compensatory mechanism to restore the energy loss in cells (Rohas et al., 2007; Garc1a-Gimenez et al., 2011; Gutsaeva et al., 2008). In this study, as an explanation for the observed PGC-1 $\alpha$ elevation, ATP levels were shown to decrease from CdTe-QD treatments at high doses. While the depletion of ATP could be 
accounted for the activation of mitochondrial biogenesis, this depletion might be a result of CdTe-QD effects on the ETC complexes that we observed in this study. CdTe-QD exposure decreased Complex I and III levels while increased Complex V level. Similarly, gene expression analyses showed that CdTe-QDs down-regulated the gene expression of Complexes I, II, III and IV, but up-regulated that of Complex V. The decreased levels and gene expression of Complexes I, II, III and IV induced by CdTeQDs indicated damage to the oxidative phosphorylation in liver cells which led to decreased ATP synthesis. On the other hand, the increased level and up-regulated gene expression of Complex V appeared to be a compensatory response of cells resulting from ATP depletion. Effects of CdTe-QDs on the ETC complexes also explained oxidative stress observed in this study. In the ETC, Complexes I and III are the sites of intracellular reactive oxygen species generation ( $\mathrm{Li}$ et al., 2003). Thus, any changes in these ETC enzymes would result in the accumulation of reactive oxygen species, which in turn leads to cellular oxidative damage and cell death. In contrast to CdTe-QDs, $\mathrm{CdCl}_{2}$ did not cause changes in mitochondrial structure and number or in the PGC-1 $\alpha$ and ATP levels. Furthermore, $\mathrm{CdCl}_{2}$ did not induce changes in the levels and gene expression of ETC Complexes I, II, III, and IV. It has been reported in previous studies that cadmium exposure also caused effects on the ETC complexes. Cadmium has been shown to inhibit the activities of Complexes I, II and III of mitochondrial ETC from isolated rainbow trout mitochondria (Adiele et al., 2012), and from guinea pig liver, brain, and heart (Belyaeva et al., 2012; Kurochokin et al., 2011; Wang et al., 2004). However, the insignificant effects of $\mathrm{CdCl}_{2}$ on the ETC as well as other mitochondria-related biomarkers in this study could be due to the used $\mathrm{CdCl}_{2}$ concentration that was insufficient to induce 
observable effects on mitochondria. These results suggested that CdTe-QDs, at an equivalent cadmium concentration, is more toxic than $\mathrm{CdCl}_{2}$ and that cadmium is not the sole factor accounting for CdTe-QDs effects as previously discussed.

In summary, the mechanisms of hepatotoxicity of CdTe-QDs in BALB/c mice were investigated in this study. The effects of these NPs on the mitochondria in the hepatocytes in the livers of treated mice was examined in details to demonstrate the roles of these organelles towards the liver injury observed from CdTe-QD treatments. Changes in mitochondrial structure, number, and function of hepatocytes in CdTe-QD exposed mice were used to explain the oxidative stress and apoptosis in the liver. Firstly, CdTe-QDs, owing to their small size and redox activity, might directly interact with mitochondrial membranes to cause change in mitochondria membrane structure and result in opening of mitochondrial permeability transition pore (PTP). PTP opening would cause releases of cytochrome $\mathrm{c}$ and Bax from the mitochondria into the cytosol and trigger the activation of apoptotic complexes leading to apoptosis. Secondly, the effects of CdTe-QDs on the ETC enzymes caused impairment of the oxidative phosphorylation process and accumulation of reactive oxygen species, which led to oxidative stress and ATP depletion. Both oxidative stress and ATP depletion might have activated the apoptotic pathways leading to cell death in the liver. Thus, mitochondria appeared to be the prominent targets for CdTe-QD hepatotoxicity, as dysfunction of these vital cell organelles results in the impairment of energy metabolism and an intracellular oxidative stress in hepatocytes in the liver of test animals. Compared with our previous in vitro study, the findings from this study highlighted the similarity in the effects and mechanisms of toxicity of CdTe-QDs in a cell line and in an animal model. Therefore, 
the study not only provides important insights into the mechanisms by which QDs exert toxicity on biological systems, but also helpful information towards bridging the gap between in vitro and in vivo assessment of the health risk associated with these NPs. 


\section{Chapter: General Discussion and Conclusion}

\subsection{Summary of research rationales, hypothesis, and approaches}

Nanotechnology has represented one of the most exciting technology developments in the last decades and will continue to provide many benefits to human society and economy for years to come (Roco, 2007). However, the potential human and environmental risks of NPs have been an issue and need to be assessed urgently. QDs, due to their unique properties, have been used in many commercial applications and for biomedical purposes (Bera et al., 2010). Without doubt, human exposures to QDs are increasing. These exposures might be from workplace, environmental (from products using QDs) and medical applications (eg. diagnostics and therapeutics). Toxicity of cadmium-based QDs has been studied in various cell and animal models; however, major uncertainties remain with regard to the toxicity and biosafety of these NPs (as reviewed Hardman, 2006; Pelley et al., 2009; Yong et al., 2013). Furthermore, information on the mechanisms underlying their toxic effects are unclear and require thorough investigation.

Regardless of the routes that QDs may enter the body to cause adverse health effects, QDs can reach the liver via the blood circulation and make it a potential target organ of the NPs (Wang et al., 2007). In addition, it has previously shown that cadmium and ROS are the likely biological factors that are responsible for cadmium-based QD induced toxicity (Derfus et al., 2004; Chang et al., 2006; Samia et al., 2006; Maysinger and Lovric, 2007; Li et al., 2009; Chen et al., 2012), and both of these factors have been shown to induce or mediate hepatotoxicity via mitochondrial pathways (Bertin and Averbeck, 2006; Samuni et al., 2010). Furthermore, mitochondrial impairment is one of the mechanisms of hepatotoxicity in cells and animals (Jaeschke et al., 2002). Therefore, 
the hypothesis of this research is that hepatotoxicity is a potential biological consequence of cadmium-based QD exposure and the effects of these NPs on mitochondria are likely to be the underlying mechanisms of hepatotoxicity.

From the proposed hypothesis, the overall objectives of this research were to examine the hepatotoxicity of CdTe-QDs in a cell line and a mouse model and to investigate the effects on mitochondria as the potential underlying mechanisms of hepatotoxicity. To achieve these objectives, the work of this research was divided into four studies, two in vitro studies (Chapter 2 and 3) and two in vivo studies (Chapter 4 and 5). The in vitro studies employed HepG2 cells as the cell model. These cells were chosen because they retain many functions of human liver cells and have high mitochondrial content (Knowles et al., 1980; Camp and Capitano, 2007). The endpoints of the in vitro studies were comprised of mechanism-driven endpoints including cytotoxicity, oxidative stress, apoptosis, and mitochondrial structure and function. Besides investigating hepatotoxicity and mitochondrial effects in HepG2 cells, the in vitro studies also allowed the selection of the appropriate endpoints, biomarkers, assays and methodologies for the entire research, which helped to better design the in vivo studies and to reduce the use of animals. The in vivo studies were performed using $\mathrm{BALB} / \mathrm{c}$ mice as the animal model as this strain has been used in numerous studies on hepatotoxicity of xenobiotics and drugs (You et al., 2006). The doses were selected within the range of those used for in vivo applications of quantum dots (Ballou et al., 2004; Gao et al., 2004; Geys et al., 2008) and were comparable to the reported doses from other in vivo studies (Haque et al., 2012; So et al., 2006; Su et al., 2011). The in vivo studies comprised of two animal testing experiments to reduce the uncertainties 
associated with exposure parameters and the overall number of used animals. While the first experiment examined the effects on mice at $24 \mathrm{~h}$, the second experiment aimed to study the effects at at longer times (ie. $3 \mathrm{~d}$ and $1 \mathrm{w}$ ) to examine the progress of the effects and the recovery of the mice from treatment. In addition to the liver function endpoints, oxidative stress, apoptosis, and mitochondrial function, systemic toxicity endpoints and biodistribution of CdTe-QDs were also investigated.

Throughout the research, test CdTe-QDs were well characterized. Characterization of NPs is essential in nanotoxicity studies as NP toxicity depends on their physical-chemical properties (Gatoo et al., 2014). In addition, characterization of NPs in a solution in which NPs are dispersed and prepared before exposure to cells or animal models provides important information on their behavior within the biological system (Boverhof and David, 2010). Characterization results can be used to predict the toxicity of the NPs and to help with the selection of appropriate endpoints for toxicological studies (Gatoo et al., 2014). In this research, before the in vitro and in vivo studies were performed, CdTe-QDs were characterized for their size, tendency agglomeration, chemical composition, and particle dissolution in different diluents including water, phosphate buffered saline (PBS), cell culture medium, saline and bovine serum albumin (BSA) (data not shown). For their size distribution and tendency of agglomeration, atomic force microscopy (AFM), transmission electron microscopy (TEM), and dynamic light scattering (DLS) were used. The size of CdTe-QDs and their tendency to agglomerate were measured and examined in the above diluents. For the composition, inductively coupled plasma optical emission spectrometry (ICP-OES) was used to assess the percentage of cadmium and telluride also the presence of any 
contaminants. The characterization by ICP-OES helped to determine the doses of these components for in vitro and in vivo experiments. Since CdTe-QDs are fluorescent, the spectral characterization of these NPs were performed in different diluents using spectrofluorometry. Information on spectral properties of test CdTe-QDs were useful for selecting appropriate kits and assays used for the entire research project. In addition, this information was also useful for the detection of CdTe-QDs in cells, tissues, and cellular components throughout the research.

It has been reported that the toxicity of QDs depends on their metal core composition and is influenced by surface coatings (Hardman, 2006). Although for cadmium-based QD toxicity, the cadmium component has received most attention due to its well-known toxicity, it is important to assess the influences of other aspects of these NPs on their biological effects. In this research the cytotoxicity of each of CdTe-QD components was assessed to compare to that of CdTe-QDs, For examples, for in vitro studies, the cytotoxicity of the polyacrylate (the polymer coating of CdTe-QDs) and of telluride (metal core component) were examined. Similarly, the cytotoxicity of mercaptopropionic (MPA), the capping material for CdTe-QDs used in vivo studies was also assessed (data not shown). For this work, these components were tested at concentrations corresponding to the cadmium and CdTe-QD concentrations used.

In cadmium-based QD structure, cadmium is the most recognized toxic component and has been assumed to be responsible for the toxicity induced by these NPs. Therefore, it is appropriate that $\mathrm{CdCl}_{2}$ was included in this research, as the control for comparing cadmium effects to those of CdTe-QDs. $\mathrm{CdCl}_{2}$ was used at concentrations that contained equivalent concentrations of cadmium to those in CdTe-QD concentrations 
tested. The employment of $\mathrm{CdCl}_{2}$ in the same assays in in vitro studies or in parallel tests in in vivo studies helped to determine whether or not the $\mathrm{Cd}$ core is responsible for CdTeQD toxicity, thus provided information on the sources of the toxicity of these NPs.

Throughout the research, assay positive controls were used in many experiments for assay validation. Similarly, the interference of CdTe-QDs with the assays employed in the in vitro and in vivo studies were assessed to eliminate the interfering artifacts. It has been suggested that NPs can interfere with several assay systems as some NP properties can hinder the outcome of an experiment through assay interference, leading to a risk of false positive or false negative results (Kroll et al., 2009). To address this issue, in this research, CdTe-QDs alone were used without sample in several assay systems to detect their potential interferences with the assays. The control experiments confirmed that QDs did not alter the background signals of the assays or inhibit their function.

\subsection{General discussion on the research outcomes}

\subsubsection{CdTe-QDs are hepatotoxins}

Hepatotoxicity caused by CdTe-QDs was investigated in vitro and in vivo in this research. Chapter 2 of this research demonstrated that these NPs induced oxidative stress leading to apoptosis and subsequent cytotoxicity in HepG2 cells. Oxidative stress was evidenced by the increased ROS production, which was accommodated by changes in antioxidant biomarkers, including GSH depletion, increased SOD activity and Nrf2 level and decreased GST and catalase activities. Oxidative stress observed in HepG2 could be the results of direct ROS generation by the NPs, as it has been suggested that these NPs can act as photosensitizers at the nanoscale (Bakalova et al., 2004). It may also from 
cadmium from the CdTe-QD core since this element has been reported to induce ROS generation in various cell lines (Almazan et al., 2000; Lopez et al., 2006). The precise mechanisms by which cadmium induces ROS generation and oxidative stress in cells or animals are not clear. However, cadmium has been shown to have high affinity for sulfhdryl (-SH) groups and can inactivate numerous antioxidant enzymes, such as catalase and GST, and sulfur-containing molecules including GSH (Lopez et al., 2006). Thus, disruption of the antioxidant systems by cadmium can result in ROS accumulation from insufficient ROS removal, leading to oxidative stress.

Intravenous administration of $\mathrm{CdTe}-\mathrm{QDs}$ into $\mathrm{BAlB} / \mathrm{c}$ mice revealed that the liver was the main target organ of these NPs. Hepatotoxicity after CdTe-QD exposure was apparent as shown by the alteration of liver morphology, including liver hemorrhage and necrosis, and changes in the levels of biomarkers of liver function. With histopathological analysis, the morphological changes in the liver induced by CdTe-QDs occurred mainly in the periportal areas of the lobules. These areas represent zone one of the liver in which hepatocytes have high concentrations of GSH (Treinen-Moslen et al., 2001). This finding suggested that oxidative stress and effects on liver antioxidant systems are likely to be key biological consequences of CdTe-QD exposure in BALB/c mice. Indeed, Chapter 5 of this research showed that CdTe-QDs resulted in oxidative stress in the liver by inducing depletion of GSH, increase in SOD activity, and upregulation of several oxidative stress related genes. Similar to the findings from the in vitro study in Chapter 2, the in vivo studies revealed that CdTe-QDs induced apoptosis in the liver in both intrinsic and extrinsic pathways. Therefore, the results from the in vitro and in vivo proved the hypothesis of this thesis that CdTe-QDs are hepatotoxins. 


\subsubsection{Cadmium is not the only factor that contributes to CdTe-QD hepatotoxicity}

Using $\mathrm{CdCl}_{2}$ as the control for examining cadmium induced effects in this research helped to elucidate the role of cadmium effects in CdTe-QD hepatotoxicity. The results from Chapter 2 showed that treatment of $\mathrm{HepG} 2$ cells with $\mathrm{CdCl}_{2}$, at an equivalent concentration of cadmium to that contained within CdTe-QDs also induced elevated ROS compared to controls, but to a lesser extent compared to CdTe-QD treatment. Similarly, for several oxidative stress and apoptosis biomarkers, the effects of $\mathrm{CdCl}_{2}$ appeared to be less compared to those of CdTe-QDs. The findings suggested that hepatotoxicity induced by CdTe-QDs in HepG2 cells was not due to cadmium alone. However, similar patterns of effects were observed between $\mathrm{CdCl}_{2}$ and $\mathrm{CdTe}-\mathrm{QDs}$, suggesting the roles of cadmium in the QD core in the hepatotoxicity induced by these NPs. For in vivo studies, using $\mathrm{CdCl}_{2}$ at an equivalent concentration of cadmium suggested that $\mathrm{CdCl}_{2}$ and $\mathrm{CdTe}$-QDs had different kinetics in BALB/c mice. At $24 \mathrm{~h}$ of exposure, due to their greater accumulation in the liver, CdTe-QDs induced more toxic effects in this organ than $\mathrm{CdCl}_{2}$. Although, kidney toxicity has been a well-known biological effects of cadmium exposures in animals and humans, effects on the kidney tend to result from chronic exposures to the metal (Friberg, 1984; Stajn et al., 1997; Roels et al., 1979; Obianime and Roberts, 2009). Data from several studies on acute toxicity of cadmium showed it is a hepatotoxin (Dudley et al., 1984; Tzirogiannis et al., 2003; Lee et al., 2009). For example, IV injection of $\mathrm{CdCl}_{2}$ in rat at $4 \mathrm{mg} / \mathrm{kg}$ bw showed significantly elevated levels of ALT and AST, central lobular necrosis around central veins, and peripheral hemorrhage around portal triads (Lee et al., 2009). However, in the current research, treatments of $\mathrm{CdCl}_{2}$ to mice did not lead to observable changes the above endpoints 
because the dose used was lower than that in this previous study. In addition, at the molecular level, there were differences in gene expression of several oxidative stress biomarker between $\mathrm{CdCl}_{2}$ and $\mathrm{CdTe}-\mathrm{QDs}$, suggesting that the underlying mechanisms and pathways of oxidative stress induced by $\mathrm{CdTe}-\mathrm{QDs}$ and $\mathrm{CdCl}_{2}$ are not identical. Similar to the in vitro studies, the results from in vivo revealed that, at an equivalent cadmium concentration, CdTe-QDs were more toxic than $\mathrm{CdCl}_{2}$, suggesting that hepatoxicity caused by CdTe-QDs was not solely due to the effects of cadmium from the core, but also resulted from the intrinsic properties of the NPs.

\subsubsection{Mitochondria are the prime targets and mitochondrial toxicity is the mechanism of hepatotoxicity caused by CdTe-QDs}

To investigate the detailed mechanisms of observed hepatotoxicity induced by CdTe-QDs in vitro and in vivo, the research went further to examine the effects of CdTeQDs on mitochondria of HepG2 cells and of hepatocytes in the liver of treated mice. Effects on mitochondrial structure and function were observed including mitochondrial enlargement, membrane potential disruption, increased intracellular calcium, ETC complexes alterations, ATP depletion, decreased cellular respiration, and mitochondrial biogenesis activation. To demonstrate the direct effects of CdTe-QDs on mitochondria in Chapter 3, the association of CdTe-QDs with mitochondria was examined by detecting CdTe-QDs in mitochondrial fractions from CdTe-QD treated cells. This finding revealed the mitochondria as the intracellular target of CdTe-QDs.

It is generally agreed that mitochondria are the major source of ROS within mammalian cells, and ROS generation is caused by electron leakage from the oxidative 
phosphorylation pathway (Andreyev et al., 2005). The most important sites of ROS formation in mitochondria are at Complexes I and III of the ETC, where electrons derived from $\mathrm{NADH}$ and ubiquinone can directly react with oxygen or other electron acceptors and generate free radicals (Adam-Vizi and Chinopoulos, 2006). ROS produced from mitochondria can react with lipids, protein, and DNA, generating oxidative damage and promoting apoptosis. Inside mitochondria, the major ROS target is the permeability transition pore (PTP). Oxidative modifications of mitochondrial PTP proteins will significantly impacts mitochondrial anion fluxes as a result of PTP opening, causing disruption of the mitochondrial membrane potential and osmotic swelling of the mitochondrial matrix (Zoratti and Szabo, 1995 and Fulda et al., 2010). Thus, in this research, the observed enlargement of mitochondria, disruption of mitochondrial membrane potential,and increased in intracellular calcium level, as the results of CdTeQD exposure, could be explained by the effects of these NPs on the ETC which led to increased ROS accumulation. Furthermore, opening of mitochondrial PTP induced by CdTe-QDs led to the release of cytochrome $\mathrm{c}$ and Bax from mitochondria as reported in Chapter 2 of this research. The release of cytochrome c has been shown to induce the formation of the Apaf-1-pro-caspase 9 apoptosome complex, which activates downstream effector caspases leading to apoptosis observed in HepG2 and in the liver of BALB/c mice.

As previously discussed, the effects on mitochondria induced by CdTe-QDs appeared as a major source of increases ROS production leading to oxidative stress in HepG2 cells and in the liver of mice in this research. However, one should not neglect the possibility that some ROS produced in HepG2 cells may also directly result from 
intact CdTe-QDs. These ROS can further cause release of cadmium from the core of CdTe-QDs and more damage to mitochondria which would lead to more production of ROS and induction of oxidative stress. Therefore, there could be three sources of ROS resulting from CdTe-QD exposure: 1) ROS from direct interaction of intact CdTe-QDs with mitochondria causing impairment of oxidative phosphorylation process; 2) ROS produced from effects of cadmium on mitochondria as well as from binding of cadmium to sulhydryl thiol groups of GSH and to antioxidant enzymes in mitochondria causing increased ROS accumulation; and 3) ROS produced directly from activation of intact CdTe-QDs within cells and mitochondria.

It has been shown that mitochondria are linked to oxidative stress and apoptotic pathways (Fariss et al., 2005; Crompton, 1999; Lakhani et al., 2006). Therefore, in this research, the interactions of CdTe-QDs and their cadmium component with mitochondria, which resulted in the damage of these organelles are likely the mechanism of oxidative stress leading to apoptosis and observed hepatotoxicity in the test models. Together, these chapters proved the hypothesis of this thesis on the roles of mitochondria in hepatotoxicity of these NPs that mitochondrial effects are the mechanism of hepatotoxicity caused by CdTe-QDs in the test models. To put them together, Figure 6.1 summarises the proposed model to highlight the mitochondrial effects of CdTe-QDs as the mechanisms of hepatotoxicity. 


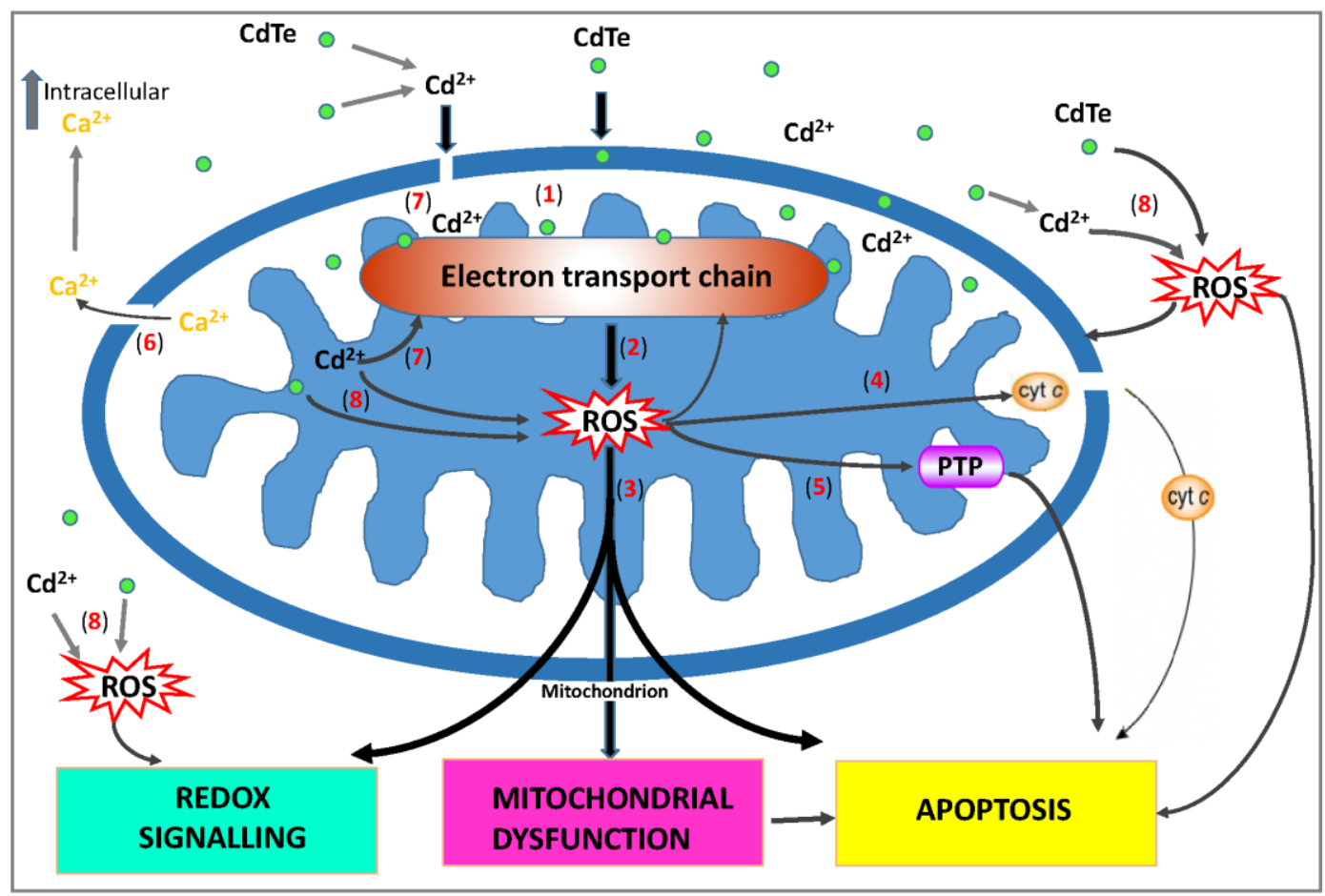

Figure 6.1. Proposed model that highlights mitochondrial effects of CdTe-QDs as the mechanisms of hepatotoxicity induced by these NPs. (1) CdTe QDs interact with the ETC leading to (2) increased ROS generation from Complex I and III, and thus (3) oxidative stress leading to redox signaling, further mitochondrial dysfunction, and apoptosis in hepatocytes. Oxidative stress results in (4) cytochrome c (cyt c) release and (5) mitochondrial pearmeability transition pore (PTP) opening, leading to apoptosis. PTP opening and disruption of mitochondrial membrane potential cause (6) the release of calcium $\left(\mathrm{Ca}^{2+}\right)$ into the cytosol leading to increased intracellular $\mathrm{Ca}^{2+}$ level. Cadmium $\left(\mathrm{Cd}^{2+}\right)$ released from CdTe-QDs can also interact with the ETC resulting in similar effects on mitochondria. (8) Both intact CdTe-QDs and $\mathrm{Cd}^{2+}$ can also induce ROS production within cells or mitochondria via redox activity, leading to redox signaling, mitochondrial dysfunction and apoptosis in cells. 


\subsection{Implications of the research}

Cadmium-based QDs are widely used in microelectronics and biomedical research, and therefore there are increasing concerns on human health and environmental risks from human exposure to these NPs and their release into the environment. Even though previous studies have reported the toxicity of cadmium-based QDs, the mechanisms related to their toxic effects are still unclear (Hardman, 2006; Pelley et al., 2009; Yong et al., 2013). Studying the hepatotoxicity of CdTe-QDs and the role of mitochondria in their toxicity will help to understand the physiological and intracellular behavior of these particles within target tissues and cells as well as the mechanism of toxicity that they exert on cells, tissues and the human body. Data obtained from this study might be helpful for classifying these nanomaterials and defining their potential risks for preliminary assessments.

Owing to their potential for health care and medical research applications, a critical assessment of risk versus benefit of use of QDs for diagnostics and therapy is extremely vital for the advancement of QD use in medicine. By identifying the sources of toxicity as well as the mechanisms of toxicity of CdTe-QDs, this research provides important information for safety evaluation of CdTe-QDs for their future biomedical applications. The information from this research might also be helpful for proper design of QDs to be safely used in the diagnostic and therapeutic applications.

It has been shown that heavy metals can cause toxicity through oxidative stress and apoptosis (Gathwan, et al., 2012). Mitochondria have been reported as important targets of many heavy metals (Belyaeva et al., 2008). This research developed important testing regimes for studying oxidative stress, apoptosis, and mitochondrial dysfunction in 
cultured cells and modeled animals. These regimes with sensitive biomarkers, assays, and methods can be useful for toxicological evaluation for other types of QDs as well as for many other metal-based nanomaterials.

In the existing literature, most studies on QD toxicity have been conducted in vitro and concern has been raised regarding the assessment of QDs using data from in vitro studies only (Hardman, 2006; Pelley et al., 2009). Since many nanomaterials including QDs tend to be organ and tissue-specific in terms of their distribution and toxicity, in vivo studies are also important for assessment of their risk in humans (Wang et al., 2013). In vitro studies on a properly selected cell line that related to organ or tissue specificity of QDs, however, can provide valuable reference information for understanding the potential toxic actions of these NPs. As previously discussed in this thesis, using HepG2 cells, which is a hepatocyte cell line, was purposeful and logical for identifying mechanisms of CdTe-QD hepatotoxicity and cellular kinetics relevant to animal and potentially human exposure. However, for assessing biosafety of nanomaterials, including QDs, information on in vitro studies is usually not sufficient as the final toxicity of nanomaterials may be dose, duration, and tissue-specific metabolism related (Wang et al., 2013). Animal studies are also capable of providing the details of both the biological and chemical fates of QDs within an intact biological system. In this research, the results from in vitro studies were validated with in vivo studies, and similar outcomes on the biological events and mechanisms were observed in the test models. Thus, this research provides helpful information towards bridging the gap between in vitro and in vivo testing for risk assessment of CdTe-QDs. 


\subsection{Conclusion and future directions}

This research studied in vitro and in vivo hepatotoxicity of CdTe-QDs and focused on investigating the effects on mitochondria as the underlying mechanisms. To achieve the objectives, the research employed HepG2 cell line and BALB/c mice, which have been shown to be relevant models for investigating hepatotoxicity, and developed testing regimes to examine mechanism-related toxicological endpoints including, oxidative stress, apoptosis, and mitochondrial dysfuntion. In HepG2 cells, hepatotoxicity induced by CdTe-QDs was evidenced by the observed cytotoxicity. On the other hand, in $\mathrm{BALB} / \mathrm{c}$ mice, liver appeared to be the main target of CdTe-QDs after IV injection, and hepatotoxicity was apparent from the observed liver injury. In addition, CdTe-QDs induced oxidative stress leading to extrinsic and intrinsic apoptosis in these models. Further to the mechanistic investigations, the research revealed mitochondria as the intracellular target of CdTe-QDs and demonstrated the effects of these NPs on mitochondrial morphology, structure and function. Using $\mathrm{CdCl}_{2}$ as the control, the research suggested that the sources of CdTe-QD toxicity were not only from cadmium component released from the core, but also from the intact CdTe-QDs and their intrinsic physicochemical properties. The interactions of cadmium and intact CdTe-QDs with mitochondria caused impairment of these organelles resulting in increased ROS

production. Since mitochondria have been linked to oxidative stress and apoptotic pathways, mitochondrial damage induced by these NPs were the mechanism of oxidative stress leading to apoptosis and observed hepatotoxicity in the test models. This research fills an important knowledge gap on the behavior of CdTe-QDs within target cells and inside biological systems as well as their underlying mechanisms of toxicity. The 
research also provides mechanistic data which is important for safety evaluation of the NPs, as well as their safe use in future biomedical applications.

Although this research investigated the detailed underlying mechanisms of CdTeQD toxicity and hepatotoxicity in vitro and in vivo, there are limitations in this work that warrant further investigation. Due to the lack of technical resources, there are questions remaining regarding characterization and detection of intact NPs within cells, mitochondria, and animals. In this research, the fluorescent property of CdTe-QDs permited some speculation of the associations of CdTe-QDs with mitochondria and tissues; however, better techniques are neccessary for quantification and detailed characterization the NPs within these biological structures in order to better correlate their presence with the observed effects presented in this research. In addition, this research focused on the effects on the liver for investigating the hepatotoxicity of CdTeQDs. For the purpose of safety assessment of these NPs in potential human applications, further studies are necessary to investigate the effects of the NPs on other tissues/organs. Furthermore, to study hepatotoxicity of CdTe-QDs in the mouse model, this research examined the acute and short term effects in these animals. Future long-term studies are needed to investigate the chronic effects in order to determine the ultimate fate of these NPs and their persistence in the animal model. 


\section{Bibliography or References}

Acin-Perez R, Salazar E, Brosel S, Yang H, Schon EA, Manfredi G. (2009). Modulation of mitochondrial protein phosphorylation by soluble adenylyl cyclase ameliorates cytochrome oxidase defects. EMBO Mol Med 1:392-406.

Adam-Vizi V, Chinopoulos C. (2006). Bioenergetics and the formation of mitochondrial reactive oxygen species. Trends Pharmacol Sci 27:639-645.

Adiele RC, Stevens D, Kamunde C. (2012). Differential inhibition of electron transport chain enzyme complexes by cadmium and calcium in isolated rainbow trout (Oncorhynchus mykiss) hepatic mitochondria. Toxicol Sci 127:110-119.

Aggarwal P, Hall JB, McLeland CB, Dobrovolskaia MA, McNeil SE. (2009). Nanoparticle interaction with plasma proteins as it relates to particle biodistribution, biocompatibility and therapeutic efficacy. Adv Drug Deliv Rev $61: 428-437$.

Allied Market Research. (2013). http://www.alliedmarketresearch.com/semiconductorand-electronics- market-report.

Alivisatos AP. (1996). Semiconductor clusters, nanocrystals, and quantum dots. Science 271:933-937

Almazan G, Liu HN, Khorchid A, Sundararajan S, Martinez-Bermudez AK, Chemtob S. (2000). Exposure of developing oligodendrocytes to cadmium causes HSP72 induction, free radical generation, reduction in glutathione levels, and cell death. Free Radic Biol Med 29:858-869.

Ambrosone A, Mattera L, Marchesano V, Quarta A, Susha AS, Tino A, Rogach AL, 
Tortiglione C. (2012). Mechanisms underlying toxicity induced by CdTe quantum dots determined in an invertebrate model organism. Biomaterials 33:1991-2000. Andreyev AY, Kushnareva YE, Starkov AA. (2005). Mitochondrial metabolism of reactive oxygen species. Biochemistry (Mosc) 70:200-214.

Armstrong JS. (2006). Mitochondrial membrane permeabilization: the sine qua non for cell death. Bioessays 28:253-260.

Aschberger K, Johnston HJ, Stone V, Aitken RJ, Hankin SM, Peters SA, Tran CL, Christensen FM. (2010). Review of carbon nanotubes toxicity and exposure-appraisal of human health risk assessment based on open literature. Crit Rev Toxicol 40:759-790.

Bakalova R, Ohba H, Zhelev Z, Ishikawa M, Baba Y. (2004). Quantum dots as photosensitizers?. Nat Biotechnol 22:1360-1361.

Ballou B, Lagerholm BC, Ernst LA, Bruchez MP, Waggoner AS. (2004). Noninvasive imaging of quantum dots in mice. Bioconjug Chem 15:79-86.

Baratli Y, Charles A-L, Wolff V, Tahar LB, Smiri L, Bouitbir J, Zoll J, Piquard F, Tebourbi O, Sakly M, Abdelmelek H, Geny B. (2013). Impact of iron oxide nanoparticles on brain, heart, lung, liver, and kidneys mitochondrial respiratory chain complexes activities. Toxicol. In Vitro 27:2142-2148.

Baroli B. (2010). Penetration of nanoparticles and nanomaterials in the skin: fiction or reality?. J Pharm Sci 99:21-50.

Bayir H, Kagan VE. (2008). Bench-to-bedside review: Mitochondrial injury, oxidative stress and apoptosis--there is nothing more practical than a good theory. Crit Care 12:206. 
Belyaeva EA, Sokolova TV, Emelyanova LV, Zakharova IO. (2012). Mitochondrial electron transport chain in heavy metal-induced neurotoxicity: effects of cadmium, mercury, and copper. ScientificWorldJournal 2012:136063.

Bera D, Qian L, Tseng T-K, Holloway PH. (2010). Quantum Dots and Their Multimodal Applications: A Review. Materials 3:2260-2345.

Berlin M, Ullberg S. (1963). The fate of Cd109 in the mouse. An autoradiographic study after a single intravenous injection of Cd109cl2. Arch Environ Health 7:686-693.

Bertin G, Averbeck D. (2006). Cadmium: cellular effects, modifications of biomolecules, modulation of DNA repair and genotoxic consequences (a review). Biochimie $88: 1549-1559$.

Bhattacharya S, Shoda LK, Zhang Q, Woods CG, Howell BA, Siler SQ, Woodhead JL, Yang Y, McMullen P, Watkins PB, Andersen ME. (2012). Modeling drug- and chemical-induced hepatotoxicity with systems biology approaches. Front Physiol $3: 462$.

Blouin A, Bolender RP, Weibel ER. (1977). Distribution of organelles and membranes between hepatocytes and nonhepatocytes in the rat liver parenchyma. A stereological study. J Cell Biol 72:441-455.

Boczkowski J, Hoet P. (2010). What's new in nanotoxicology? Implications for public health from a brief review of the 2008 literature. Nanotoxicology 4:1-14.

Bolduc JS, Denizeau F, Jumarie C. (2004). Cadmium-induced mitochondrial membranepotential dissipation does not necessarily require cytosolic oxidative stress: studies using rhodamine-123 fluorescence unquenching. Toxicol Sci 77:299-306. 
Boverhof DR, David RM. (2010). Nanomaterial characterization: considerations and needs for hazard assessment and safety evaluation. Anal Bioanal Chem 396:953961.

Brand MD, Nicholls DG. (2011). Assessing mitochondrial dysfunction in cells. Biochem J 435:297-312.

Brown GC. (1992). Control of respiration and ATP synthesis in mammalian mitochondria and cells. Biochem J 284 ( Pt 1):1-13.

Bruchez M,Jr, Moronne M, Gin P, Weiss S, Alivisatos AP. (1998). Semiconductor nanocrystals as fluorescent biological labels. Science 281:2013-2016.

Bruneau A, Fortier M, Gagne F, Gagnon C, Turcotte P, Tayabali A, Davis TA, Auffret M, Fournier M. (2015). In vitro immunotoxicology of quantum dots and comparison with dissolved cadmium and tellurium. Environ Toxicol 30:9-25.

BSI. (2011). Nanoparticles - Vocabulary (PAS 71:2011). London:British Standards Institution.

Buttke TM, Sandstrom PA. (1994). Oxidative stress as a mediator of apoptosis. Immunol Today 15:7-10.

Byerrum RU, Decker CF, Hoppert CA. (1957). A study of the distribution and retention of cadmium-115 in the albino rat. Arch Biochem Biophys 66:140-145.

Cai W, Hsu AR, Li ZB, Chen X. (2007). Are quantum dots ready for in vivo imaging in human subjects?. Nanoscale Res Lett 2:265-281.

Cai W, Shin DW, Chen K, Gheysens O, Cao Q, Wang SX, Gambhir SS, Chen X. (2006). Peptide-labeled near-infrared quantum dots for imaging tumor vasculature in living subjects. Nano Lett 6:669-676. 
Camp JP, Capitano AT. (2007). Induction of zone-like liver function gradients in HepG2 cells by varying culture medium height. Biotechnol Prog 23:1485-1491.

Carmody RJ, Cotter TG. (2001). Signalling apoptosis: a radical approach. Redox Rep 6:77-90.

Cesar CL. (2014). Quantum dots as biophotonics tools. Methods Mol Biol 1199:3-9.

Chan WC, Maxwell DJ, Gao X, Bailey RE, Han M, Nie S. (2002). Luminescent quantum dots for multiplexed biological detection and imaging. Curr Opin Biotechnol $13: 40-46$

Chan WH, Shiao NH, Lu PZ. (2006). CdSe quantum dots induce apoptosis in human neuroblastoma cells via mitochondrial-dependent pathways and inhibition of survival signals. Toxicol Lett 167:191-200.

Chang E, Thekkek N, Yu WW, Colvin VL, Drezek R. (2006). Evaluation of quantum dot cytotoxicity based on intracellular uptake. Small 2:1412-1417.

Chelikani P, Fita I, Loewen PC. (2004). Diversity of structures and properties among catalases. Cell Mol Life Sci 61:192-208.

Chen LB. (1988). Mitochondrial membrane potential in living cells. Annu Rev Cell Biol 4:155-181.

Chen N, He Y, Su Y, Li X, Huang Q, Wang H, Zhang X, Tai R, Fan C. (2012). The cytotoxicity of cadmium-based quantum dots. Biomaterials 33:1238-1244.

Chen Z, Chen H, Meng H, Xing G, Gao X, Sun B, Shi X, Yuan H, Zhang C, Liu R, Zhao F, Zhao Y, Fang X. (2008). Bio-distribution and metabolic paths of silica coated CdSeS quantum dots. Toxicol Appl Pharmacol 230:364-371.

Chen Z, Meng H, Xing G, Chen C, Zhao Y, Jia G, Wang T, Yuan H, Ye C, Zhao F, Chai 
Z, Zhu C, Fang X, Ma B, Wan L. (2006). Acute toxicological effects of copper nanoparticles in vivo. Toxicol Lett 163:109-120.

Chinopoulos C, Adam-Vizi V. (2006). Calcium, mitochondria and oxidative stress in neuronal pathology. Novel aspects of an enduring theme. FEBS J 273:433-450.

Cho SJ, Maysinger D, Jain M, Roder B, Hackbarth S, Winnik FM. (2007). Long-term exposure to CdTe quantum dots causes functional impairments in live cells. Langmuir 23:1974-1980.

Choi AO, Cho SJ, Desbarats J, Lovric J, Maysinger D. (2007). Quantum dot-induced cell death involves Fas upregulation and lipid peroxidation in human neuroblastoma cells. J Nanobiotechnology 5:1.

Clift MJ, Boyles MS, Brown DM, Stone V. (2010). An investigation into the potential for different surface-coated quantum dots to cause oxidative stress and affect macrophage cell signalling in vitro. Nanotoxicology 4:139-149.

Crompton M. (2000). Bax, Bid and the permeabilization of the mitochondrial outer membrane in apoptosis. Curr Opin Cell Biol 12:414-419.

Crompton M. (1999). The mitochondrial permeability transition pore and its role in cell death. Biochem J 341 ( Pt 2):233-249.

Daniel SP, Marshall MK. (1999). Evaluation of the liver: laboratory tests. Schiff's diseases of the liver, 8th edn, JB Lippincott publications. pp. 205-239.

De Wild M, Berner S, Suzuki H, Ramoino L, Baratoff A, Jung TA. (2003). Molecular assembly and self-assembly: molecular nanoscience for future technologies. Ann N Y Acad Sci 1006:291-305.

Dejean LM, Martinez-Caballero S, Manon S, Kinnally KW. (2006). Regulation of the 
mitochondrial apoptosis-induced channel, MAC, by BCL-2 family proteins. Biochim Biophys Acta 1762:191-201.

Derfus AM, Chan WCW, Bhatia SN. (2004). Probing the cytotoxicity of semiconductor Quantum Dots. Nano Lett 4:11-18.

Dierickx PJ. (1982). In vitro inhibition of the soluble glutathione S-transferases from rat liver by heavy metals. Enzyme 27:25-32.

Donaldson K, Stone V, Clouter A, Renwick L, MacNee W. (2001). Ultrafine particles. Occup Environ Med 58:211-6, 199.

Donaldson K, Stone V, Tran CL, Kreyling W, Borm PJ. (2004). Nanotoxicology. Occup Environ Med 61:727-728.

Dreher KL. (2004). Health and environmental impact of nanotechnology: toxicological assessment of manufactured nanoparticles. Toxicol Sci 77:3-5.

Drose S, Brandt U. (2008). The mechanism of mitochondrial superoxide production by the cytochrome bc1 complex. J Biol Chem 283:21649-21654.

Duchen MR. (2004). Mitochondria in health and disease: perspectives on a new mitochondrial biology. Mol Aspects Med 25:365-451.

Duchen MR. (2000). Mitochondria and calcium: from cell signalling to cell death. J Physiol 529 Pt 1:57-68.

Dudley RE, Svoboda DJ, Klaassen CD. (1984). Time course of cadmium-induced ultrastructural changes in rat liver. Toxicol Appl Pharmacol 76:150-160.

Dyce KM, Sack WO, Wensing CJ. (1987). Text Book of Veterinary Anatomy. W B Saunders Co, Philadelphia. 542-595.

Erogbogbo F, Yong KT, Roy I, Hu R, Law WC, Zhao W, Ding H, Wu F, Kumar R, 
Swihart MT, Prasad PN. (2011). In vivo targeted cancer imaging, sentinel lymph node mapping and multi-channel imaging with biocompatible silicon nanocrystals. ACS Nano 5:413-423.

Fariss MW, Chan CB, Patel M, Van Houten B, Orrenius S. (2005). Role of mitochondria in toxic oxidative stress. Mol Interv 5:94-111.

Farre M, Sanchis J, Barcelo D. (2009). Analysis and assessment of the occurrence, the fate and the behaviour of nanomaterials in the environment. Trends Anal Chem $30(3): 517-527$.

Fas SC, Baumann S, Krueger A, Frey CR, Schulze-Bergkamen H, Brenner D, Stumpf C, Kappes K, Krammer PH. (2006). In vitro generated human memory-like T cells are CD95 type II cells and resistant towards CD95-mediated apoptosis. Eur J Immunol 36:2894-2903.

Finucane DM, Bossy-Wetzel E, Waterhouse NJ, Cotter TG, Green DR. (1999). Baxinduced caspase activation and apoptosis via cytochrome c release from mitochondria is inhibitable by Bcl-xL. J Biol Chem 274:2225-2233.

Fischer HC, Liu L, Pang KS, Chan WCW. (2006). Pharmacokinetics of nanoscale quantum dots: in vivo distribution, sequestration, and clearance in the rat. Advanced Functional Materials 16 (10): 1299-1305.

Foley S, Crowley C, Smaihi M, Bonfils C, Erlanger BF, Seta P, Larroque C. (2002). Cellular localisation of a water-soluble fullerene derivative. Biochem Biophys Res Commun 294:116-119.

Frezza C, Cipolat S, Scorrano L. (2007). Organelle isolation: functional mitochondria from mouse liver, muscle and cultured fibroblasts. Nat Protoc 2:287-295. 
Friberg L. (1984). Cadmium and the kidney. Environ Health Perspect 54:1-11.

Fulda S, Debatin KM. (2006). Extrinsic versus intrinsic apoptosis pathways in anticancer chemotherapy. Oncogene 25:4798-4811.

Fulda S, Debatin KM. (2006). Modulation of apoptosis signaling for cancer therapy. Arch Immunol Ther Exp (Warsz) 54:173-175.

Fulda S, Galluzzi L, Kroemer G. (2010). Targeting mitochondria for cancer therapy. Nat Rev Drug Discov 9:447-464.

Gagne F, Auclair J, Turcotte P, Fournier M, Gagnon C, Sauve S, Blaise C. (2008).

Ecotoxicity of CdTe quantum dots to freshwater mussels: impacts on immune system, oxidative stress and genotoxicity. Aquat Toxicol 86:333-340.

Gagne F, Fortier M, Yu L, Osachoff HL, Skirrow RC, van Aggelen G, Gagnon C, Fournier M. (2010). Immunocompetence and alterations in hepatic gene expression in rainbow trout exposed to $\mathrm{CdS} / \mathrm{CdTe}$ quantum dots. J Environ Monit $12: 1556-1565$.

Galante YM, Wong SY, Hatefi Y. (1979). Composition of complex V of the mitochondrial oxidative phosphorylation system. J Biol Chem 254:12372-12378.

Gao X, Cui Y, Levenson RM, Chung LW, Nie S. (2004). In vivo cancer targeting and imaging with semiconductor quantum dots. Nat Biotechnol 22:969-976.

Garcia-Gimenez JL, Gimeno A, Gonzalez-Cabo P, Dasi F, Bolinches-Amoros A, Molla B, Palau F, Pallardo FV. (2011). Differential expression of PGC-1alpha and metabolic sensors suggest age-dependent induction of mitochondrial biogenesis in Friedreich ataxia fibroblasts. PLoS One 6:e20666.

Gathwan KH, QM Ali Al Ameri, Zaidan HK. (2012). Metals induce apoptosis in liver of 
mice. Int J Appl Biol Pharmaceut Technol 3(2):146-150.

Gatoo MA, Naseem S, Arfat MY, Dar AM, Qasim K, Zubair S. (2014). Physicochemical properties of nanomaterials: implication in associated toxic manifestations. Biomed Res Int 2014:498420.

Gerion D, Pinaud F, Williams SC, Parak WJ, Zanchet D, Weiss S, Alivisatos AP. (2001). Synthesis and properties of biocompatible water-soluble silica-coated $\mathrm{CdSe} / \mathrm{ZnS}$ semiconductor quantum dots. J Phys Chem B 105:8861-8871467

Geys J, Nemmar A, Verbeken E, Smolders E, Ratoi M, Hoylaerts MF, Nemery B, Hoet PH. (2008). Acute toxicity and prothrombotic effects of quantum dots: impact of surface charge. Environ Health Perspect 116:1607-1613.

Gottlieb E, Armour SM, Harris MH, Thompson CB. (2003). Mitochondrial membrane potential regulates matrix configuration and cytochrome $\mathrm{c}$ release during apoptosis. Cell Death Differ 10:709-717.

Green DR, Reed JC. (1998). Mitochondria and apoptosis. Science 281:1309-1312.

Guo G, Liu W, Liang J, He Z, Xu H, Yang X. (2007). Probing the cytotoxicity of CdSe quantum dots with surface modification. Mater Lett 61:1641-1644.

Gutsaeva DR, Carraway MS, Suliman HB, Demchenko IT, Shitara H, Yonekawa H, Piantadosi CA. (2008). Transient hypoxia stimulates mitochondrial biogenesis in brain subcortex by a neuronal nitric oxide synthase-dependent mechanism. $\mathrm{J}$ Neurosci 28:2015-2024.

Hahn MA, Singh AK, Sharma P, Brown SC, Moudgil BM. (2011). Nanoparticles as contrast agents for in-vivo bioimaging: current status and future perspectives. Anal Bioanal Chem 399:3-27. 
Hamada T, Tanimoto A, Sasaguri Y. (1997). Apoptosis induced by cadmium. Apoptosis 2:359-367.

Haque M M, Im H-Y, Seo J-E, Hasan M, Sarma S N, Woo K, Kwon O-S. (2012).

Evaluation of CdSe/CdS-PEG-FA quantum dots: distribution and observableadverse-effect-level in mice after intravenous injection. J. Pharm. Invest 42 (4): 203-212.

Haque MM, Im HY, Seo JE, Hasan M, Woo K, Kwon OS. (2013). Acute toxicity and tissue distribution of $\mathrm{CdSe} / \mathrm{CdS}-\mathrm{MPA}$ quantum dots after repeated intraperitoneal injection to mice. J Appl Toxicol 33:940-950.

Hardman R. (2006). A toxicologic review of quantum dots: toxicity depends on physicochemical and environmental factors. Environ Health Perspect 114:165172.

Harrison MT, Kershaw SV, Burt MG, Rogach AL, Kornowski A, Eychmuller A, Weller H. (2000). Colloidal nanocrystals for telecommunications. Complete coverage of the low loss fiber windows by mercury telluride quantum dots. Pure Appl Chem 72:295-307.

Hauck TS, Anderson RE, Fischer HC, Newbigging S, Chan WC. (2010). In vivo quantum-dot toxicity assessment. Small 6:138-144.

Hayes JD, McLellan LI. (1999). Glutathione and glutathione-dependent enzymes represent a co-ordinately regulated defence against oxidative stress. Free Radic Res 31:273-300.

Hetsch F, Xu XQ, Wang HK, Kershaw SV, Rogach AL. (2011). Semiconductor 
nanocrystal quantum dots as solar cell components and photosensitizers: material, charge transfer, and separation aspects of some device topologies. J Phys Chem Lett 2:1879-1887.

Hillyer JF, Albrecht RM. (2001). Gastrointestinal persorption and tissue distribution of differently sized colloidal gold nanoparticles. J Pharm Sci 90:1927-1936.

Hoshino A, Fujioka K, Oku T, Nakamura S, Suga M, Yamaguchi Y, Suzuki K, Yasuhara M, Yamamoto K. (2004). Quantum dots targeted to the assigned organelle in living cells. Microbiol Immunol 48:985-994.

Hug H, Enari M, Nagata S. (1994). No requirement of reactive oxygen intermediates in Fas-mediated apoptosis. FEBS Lett 351:311-313.

Hussain SM, Hess KL, Gearhart JM, Geiss KT, Schlager JJ. (2005). In vitro toxicity of nanoparticles in BRL 3A rat liver cells. Toxicol In Vitro 19:975-983.

Hynes J, Marroquin LD, Ogurtsov VI, Christiansen KN, Stevens GJ, Papkovsky DB, Will Y. (2006). Investigation of drug-induced mitochondrial toxicity using fluorescence-based oxygen-sensitive probes. Toxicol Sci 92:186-200.

Irache JM, Esparza I, Gamazo C, Agueros M, Espuelas S. (2011). Nanomedicine: novel approaches in human and veterinary therapeutics. Vet Parasitol 180:47-71.

ISO. (2010). Nanotechnologies - Vocabulary - Part 1: Core Terns. (TS 80004-1).Geneva, Switzerland:International Organization for Standardization.

Jain TK, Reddy MK, Morales MA, Leslie-Pelecky DL, Labhasetwar V. (2008).

Biodistribution, clearance, and biocompatibility of iron oxide magnetic nanoparticles in rats. Mol Pharm 5:316-327.

Jamieson T, Bakhshi R, Petrova D, Pocock R, Imani M, Seifalian AM. (2007). Biological 
applications of quantum dots. Biomaterials 28:4717-4732.

Jin T, Lu J, Nordberg M. (1998). Toxicokinetics and biochemistry of cadmium with special emphasis on the role of metallothionein. Neurotoxicology 19:529-535.

Jornayvaz FR, Shulman GI. (2010). Regulation of mitochondrial biogenesis. Essays Biochem 47:69-84.

Juzenas P, Chen W, Sun YP, Coelho MA, Generalov R, Generalova N, Christensen IL. (2008). Quantum dots and nanoparticles for photodynamic and radiation therapies of cancer. Adv Drug Deliv Rev 60:1600-1614.

Kaplowitz N. (2004). Drug-induced liver injury. Clin Infect Dis 38 Suppl 2:S44-8.

Kato S, Itoh K, Yaoi T, Tozawa T, Yoshikawa Y, Yasui H, Kanamura N, Hoshino A, Manabe N, Yamamoto K, Fushiki S. (2010). Organ distribution of quantum dots after intraperitoneal administration, with special reference to area-specific distribution in the brain. Nanotechnology 21:335103.

Kato T, Ogami K, Shimada Y, Iwamatsu A, Sohma Y, Akahori H, Horie K, Kokubo A, Kudo Y, Maeda E. (1995). Purification and characterization of thrombopoietin. J Biochem 118:229-236.

Kim BJ, Ryu SW, Song BJ. (2006). JNK- and p38 kinase-mediated phosphorylation of Bax leads to its activation and mitochondrial translocation and to apoptosis of human hepatoma HepG2 cells. J Biol Chem 281:21256-21265.

Kim J, Park Y, Yoon TH, Yoon CS, Choi K. (2010). Phototoxicity of CdSe/ZnSe quantum dots with surface coatings of 3-mercaptopropionic acid or tri-noctylphosphine oxide/gum arabic in Daphnia magna under environmentally relevant UV-B light. Aquat Toxicol 97:116-124. 
Kim S, Fisher B, Eisler HJ, Bawendi M. (2003). Type-II quantum dots:

$\mathrm{CdTe} / \mathrm{CdSe}$ (core/shell) and $\mathrm{CdSe} / \mathrm{ZnTe}$ (core/shell) heterostructures. J Am Chem Soc 125:11466-11467.

King-Heiden TC, Wiecinski PN, Mangham AN, Metz KM, Nesbit D, Pedersen JA, Hamers RJ, Heideman W, Peterson RE. (2009). Quantum dot nanotoxicity assessment using the zebrafish embryo. Environ Sci Technol 43:1605-1611.

Kirchner C, Liedl T, Kudera S, Pellegrino T, Munoz Javier A, Gaub HE, Stolzle S, Fertig N, Parak WJ. (2005). Cytotoxicity of colloidal CdSe and CdSe/ZnS nanoparticles. Nano Lett 5:331-338.

Knasmuller S, Mersch-Sundermann V, Kevekordes S, Darroudi F, Huber WW, Hoelzl C, Bichler J, Majer BJ. (2004). Use of human-derived liver cell lines for the detection of environmental and dietary genotoxicants; current state of knowledge. Toxicology 198:315-328.

Knowles BB, Howe CC, Aden DP. (1980). Human hepatocellular carcinoma cell lines secrete the major plasma proteins and hepatitis B surface antigen. Science 209:497-499.

Knutti D, Kralli A. (2001). PGC-1, a versatile coactivator. Trends Endocrinol Metab $12: 360-365$

Kondoh M, Araragi S, Sato K, Higashimoto M, Takiguchi M, Sato M. (2002). Cadmium induces apoptosis partly via caspase-9 activation in HL-60 cells. Toxicology 170:111-117.

Kotsonis FN, Klaassen CD. (1977). Toxicity and distribution of cadmium administered to rats at sublethal doses. Toxicol Appl Pharmacol 41:667-680. 
Kroll A, Pillukat MH, Hahn D, Schnekenburger J. (2009). Current in vitro methods in nanoparticle risk assessment: limitations and challenges. Eur J Pharm Biopharm $72: 370-377$

Kuester RK, Waalkes MP, Goering PL, Fisher BL, McCuskey RS, Sipes IG. (2002). Differential hepatotoxicity induced by cadmium in Fischer 344 and SpragueDawley rats. Toxicol Sci 65:151-159.

Kurochkin IO, Etzkorn M, Buchwalter D, Leamy L, Sokolova IM. (2011). Top-down control analysis of the cadmium effects on molluscan mitochondria and the mechanisms of cadmium-induced mitochondrial dysfunction. Am J Physiol Regul Integr Comp Physiol 300:R21-31.

Lacerda SH, Park JJ, Meuse C, Pristinski D, Becker ML, Karim A, Douglas JF. (2010). Interaction of gold nanoparticles with common human blood proteins. ACS Nano 4:365-379.

Lai DY. (2012). Toward toxicity testing of nanomaterials in the 21 st century: a paradigm for moving forward. Wiley Interdiscip Rev Nanomed Nanobiotechnol 4:1-15.

Lakhani SA, Masud A, Kuida K, Porter GA,Jr, Booth CJ, Mehal WZ, Inayat I, Flavell RA. (2006). Caspases 3 and 7: key mediators of mitochondrial events of apoptosis. Science 311:847-851.

Law WC, Yong KT, Roy I, Ding H, Hu R, Zhao W, Prasad PN. (2009). Aqueous-phase synthesis of highly luminescent $\mathrm{CdTe} / \mathrm{ZnTe}$ core/shell quantum dots optimized for targeted bioimaging. Small 5:1302-1310.

Lee J, Ji K, Kim J, Park C, Lim KH, Yoon TH, Choi K. (2010). Acute toxicity of two 
CdSe/ZnSe quantum dots with different surface coating in Daphnia magna under various light conditions. Environ Toxicol 25:593-600.

Lee JR, Park SJ, Lee HS, Jee SY, Seo J, Kwon YK, Kwon TK, Kim SC. (2009).

Hepatoprotective activity of licorice water extract against cadmium-induced toxicity in rats. Evid Based Complement Alternat Med 6:195-201.

Levi AJ, Gatmaitan Z, Arias IM. (1969). Two hepatic cytoplasmic protein fractions, Y and $\mathrm{Z}$, and their possible role in the hepatic uptake of bilirubin, sulfobromophthalein, and other anions. J Clin Invest 48:2156-2167.

Li J, Zhang Y, Xiao Q, Tian F, Liu X, Li R, Zhao G, Jiang F, Liu Y. (2011).

Mitochondria as target of quantum dots toxicity. J Hazard Mater 194:440-444.

Li KG, Chen JT, Bai SS, Wen X, Song SY, Yu Q, Li J, Wang YQ. (2009). Intracellular oxidative stress and cadmium ions release induce cytotoxicity of unmodified cadmium sulfide quantum dots. Toxicol In Vitro 23:1007-1013.

Li N, Sioutas C, Cho A, Schmitz D, Misra C, Sempf J, Wang M, Oberley T, Froines J, Nel A. (2003). Ultrafine particulate pollutants induce oxidative stress and mitochondrial damage. Environ Health Perspect 111:455-460.

Li Y, Lim SC. (2007). Cadmium-induced apoptosis of hepatocytes is not associated with death receptor-related caspase-dependent pathways in the rat. Environ Toxicol Pharmacol 24:231-238.

Liang XW, Xu ZP, Grice J, Zvyagin AV, Roberts MS, Liu X. (2013). Penetration of nanoparticles into human skin. Curr Pharm Des 19:6353-6366.

Lin CH, Chang LW, Wei YH, Wu SB, Yang CS, Chang WH, Chen YC, Lin PP. (2012). 
Electronic microscopy evidence for mitochondria as targets for $\mathrm{Cd} / \mathrm{Se} / \mathrm{Te}$-based quantum dot 705 toxicity in vivo. Kaohsiung J Med Sci 28:S53-62.

Lin CH, Yang MH, Chang LW, Yang CS, Chang H, Chang WH, Tsai MH, Wang CJ, Lin P. (2011). Cd/Se/Te-based quantum dot 705 modulated redox homeostasis with hepatotoxicity in mice. Nanotoxicology 5:650-663.

Liu T, He W, Yan C, Qi Y, Zhang Y. (2011). Roles of reactive oxygen species and mitochondria in cadmium-induced injury of liver cells. Toxicol Ind Health 27:249-256.

Liu W, Zhang S, Wang L, Qu C, Zhang C, Hong L, Yuan L, Huang Z, Wang Z, Liu S, Jiang G. (2011). CdSe quantum dot (QD)-induced morphological and functional impairments to liver in mice. PLoS One 6:e24406.

Lopez E, Arce C, Oset-Gasque MJ, Canadas S, Gonzalez MP. (2006). Cadmium induces reactive oxygen species generation and lipid peroxidation in cortical neurons in culture. Free Radic Biol Med 40:940-951.

Lovric J, Bazzi HS, Cuie Y, Fortin GR, Winnik FM, Maysinger D. (2005b). Differences in subcellular distribution and toxicity of green and red emitting CdTe quantum dots. J Mol Med (Berl) 83:377-385.

Lovric J, Cho SJ, Winnik FM, Maysinger D. (2005a). Unmodified cadmium telluride quantum dots induce reactive oxygen species formation leading to multiple organelle damage and cell death. Chem Biol 12:1227-1234. 
Lu HY, Shiao NH, Chan WH. (2006). CdSe quantum dots induce apoptosis via activation of JNK and PAK2 in a human osteoblast cell line. J Med Biol Eng 26:89-96.

Luo X, Budihardjo I, Zou H, Slaughter C, Wang X. (1998). Bid, a Bcl2 interacting protein, mediates cytochrome c release from mitochondria in response to activation of cell surface death receptors. Cell 94:481-490.

MacSween RNM, Desmet VJ, Roskams T, Scothorne RJ. (2002). Developmental anatomy and normal structure. In Pathology of the Liver. Churchill Livingstone, New York. 1-66.

Mao WP, Zhang NN, Zhou FY, Li WX, Liu HY, Feng J, Zhou L, Wei CJ, Pan YB, He ZJ. (2011). Cadmium directly induced mitochondrial dysfunction of human embryonic kidney cells. Hum Exp Toxicol 30:920-929.

Martel J, Marion M, Denizeau F. (1990). Effect of cadmium on membrane potential in isolated rat hepatocytes. Toxicology 60:161-172.

Martin P, Pognonec P. (2010). ERK and cell death: cadmium toxicity, sustained ERK activation and cell death. FEBS J 277:39-46.

Maysinger D, Lovric J. (2007). Quantum dots and other fluorescent nanoparticles: quo vadis in the cell? Adv Exp Med Biol 620:156-167.

McBride HM, Neuspiel M, Wasiak S. (2006). Mitochondria: more than just a powerhouse. Curr Biol 16:R551-60.

Medintz IL, Mattoussi H, Clapp AR. (2008). Potential clinical applications of quantum dots. Int J Nanomedicine 3:151-167.

Michalet X, Pinaud FF, Bentolila LA, Tsay JM, Doose S, Li JJ, Sundaresan G, Wu AM, 
Gambhir SS, Weiss S. (2005). Quantum dots for live cells, in vivo imaging, and diagnostics. Science 307:538-544.

Mitchell P. (1967). Proton current flow in mitochondrial systems. Nature 214:1327-1328.

Mitchell P. (1961). Coupling of phosphorylation to electron and hydrogen transfer by a chemi-osmotic type of mechanism. Nature 191:144-148.

Mukundan H, Xie H, Price D, Kubicek-Sutherland JZ, Grace WK, Anderson AS, Martinez JS, Hartman N, Swanson BI. (2010). Quantitative multiplex detection of pathogen biomarkers on multichannel waveguides. Anal Chem 82:136-144.

Narita R, Asaumi H, Abe S, Nakamura H, Tabaru A, Yoshikawa I, Yamada S, Otsuki M. (2003). Idiopathic thrombocytopenic purpura with acute hepatitis C viral infection. J Gastroenterol Hepatol 18:462-463.

Nel A, Xia T, Madler L, Li N. (2006). Toxic potential of materials at the nanolevel. Science 311:622-627.

Neupert W, Herrmann JM. (2007). Translocation of proteins into mitochondria. Annu Rev Biochem 76:723-749.

Nguyen KC, Rippstein P, Tayabali AF, Willmore WG. (2015). Mitochondrial toxicity of cadmium telluride quantum dot nanoparticles in mammalian hepatocytes.

Toxicol Sci 146(1):31-42.

Nguyen KC, Seligy VL, Tayabali AF. (2013a). Cadmium telluride quantum dot nanoparticle cytotoxicity and effects on model immune responses to Pseudomonas aeruginosa. Nanotoxicology 7:202-211.

Nguyen KC, Willmore WG, Tayabali AF. (2013b). Cadmium telluride quantum dots 
cause oxidative stress leading to extrinsic and intrinsic apoptosis in hepatocellular carcinoma HepG2 cells. Toxicology 306:114-123.

Nowack B, Bucheli TD. (2007). Occurrence, behavior and effects of nanoparticles in the environment. Environ Pollut 150:5-22.

Oberdorster G, Maynard A, Donaldson K, Castranova V, Fitzpatrick J, Ausman K, Carter J, Karn B, Kreyling W, Lai D, Olin S, Monteiro-Riviere N, Warheit D, Yang H, ILSI Research Foundation/Risk Science Institute Nanomaterial Toxicity Screening Working Group. (2005). Principles for characterizing the potential human health effects from exposure to nanomaterials: elements of a screening strategy. Part Fibre Toxicol 2:8.

Obianime WA, Roberts II. (2009). Antioxidants, cadmium-induced toxicity, serum biochemical and the histological abnormalities of the kidney and testes of the male wistar rats. Niger J Physiol Sci 24(2):177-185.

Oh SH, Lim SC. (2006). A rapid and transient ROS generation by cadmium triggers apoptosis via caspase-dependent pathway in HepG2 cells and this is inhibited through N-acetylcysteine-mediated catalase upregulation. Toxicol Appl Pharmacol 212:212-223.

Osmond MJ, McCall MJ. (2010). Zinc oxide nanoparticles in modern sunscreens: an analysis of potential exposure and hazard. Nanotoxicology 4:15-41.

Pace HE, Lesher EK, Ranville JF. (2010). Influence of stability on the acute toxicity of CdSe/ZnS nanocrystals to Daphnia magna. Environ Toxicol Chem 29:1338-1344. 
Pandit A, Sachdeva T, Bafna P. (2012). Drug-induced hepatotoxicity: A review. J Appl Pharm Sci 02(05):233-243.

Passarella S, Atlante A, Valenti D, de Bari L. (2003). The role of mitochondrial transport in energy metabolism. Mitochondrion 2:319-343.

Patel IR, Patel M, Geeta P, Patel GN, Patel NJ. (2007). Quantum dots: A novel technique for drug delivery and therapy. Pharmaceutical Reviews 01/2007.

Pelley JL, Daar AS, Saner MA. (2009). State of academic knowledge on toxicity and biological fate of quantum dots. Toxicol Sci 112:276-296.

Petit PX, Lecoeur H, Zorn E, Dauguet C, Mignotte B, Gougeon ML. (1995). Alterations in mitochondrial structure and function are early events of dexamethasoneinduced thymocyte apoptosis. J Cell Biol 130:157-167.

Peyrot C, Gagnon C, Gagne F, Willkinson KJ, Turcotte P, Sauve S. (2009). Effects of cadmium telluride quantum dots on cadmium bioaccumulation and metallothionein production to the freshwater mussel, Elliptio complanata. Comp Biochem Physiol C Toxicol Pharmacol 150:246-251.

Pinti M, Troiano L, Nasi M, Ferraresi R, Dobrucki J, Cossarizza A. (2003). Hepatoma HepG2 cells as a model for in vitro studies on mitochondrial toxicity of antiviral drugs: which correlation with the patient? J Biol Regul Homeost Agents 17:166171.

Pruell RJ, Engelhardt FR. (1980). Liver cadmium uptake, catalase inhibition and cadmium thionein production in the killifish (Fundulus Heteroclitus) induced by experimental cadmium exposure. Mar Environ Res 3:101-111.

Putcha GV, Harris CA, Moulder KL, Easton RM, Thompson CB, Johnson EM,Jr. (2002). 
Intrinsic and extrinsic pathway signaling during neuronal apoptosis: lessons from the analysis of mutant mice. J Cell Biol 157:441-453.

Qian L, Zheng Y, Xue JG, Holloway PH. (2011). Stable and efficient quantum-dot lightemitting diodes based on solution-processed multilayer structures. Nat Photonics $5: 543-548$.

Rios R, Sangro B, Herrero I, Quiroga J, Prieto J. (2005). The role of thrombopoietin in the thrombocytopenia of patients with liver cirrhosis. Am J Gastroenterol 100:1311-1316.

Rocha TL, Gomes T, Cardoso C, Letendre J, Pinheiro JP, Sousa VS, Teixeira MR, Bebianno MJ. (2014). Immunocytotoxicity, cytogenotoxicity and genotoxicity of cadmium-based quantum dots in the marine mussel Mytilus galloprovincialis. Mar Environ Res 101:29-37.

Roco MC. (2007). The NNI: past, present and future. Goddard, W.A et al. (ed.) Handbook on Nanoscience, Engineering and Technology, CRC, Taylor and Francis, Boca Raton and London. 3.1-3.26.

Roco MC. (2011). The long view of nanotechnology development: the National Nanotechnology Initiative at 10 years. J Nanopart Res 13(2):427-445.

Rodrigo GC, Standen NB. (2005). Role of mitochondrial re-energization and Ca2+ influx in reperfusion injury of metabolically inhibited cardiac myocytes. Cardiovasc Res 67:291-300.

Roels H, Bernard A, Buchet JP, Goret A, Lauwerys R, Chettle DR, Harvey TC, Haddad IA. (1979). Critical concentration of cadmium in renal cortex and urine. Lancet $1: 221$. 
Rohas LM, St-Pierre J, Uldry M, Jager S, Handschin C, Spiegelman BM. (2007). A fundamental system of cellular energy homeostasis regulated by PGC-1alpha. Proc Natl Acad Sci U S A 104:7933-7938.

Ryan JA, Pahren HR, Lucas JB. (1982). Controlling cadmium in the human food chain: a review and rationale based on health effects. Environ Res 28:251-302.

Rzigalinski BA, Strobl JS. (2009). Cadmium-containing nanoparticles: perspectives on pharmacology and toxicology of quantum dots. Toxicol Appl Pharmacol 238:280288.

Sadauskas E, Wallin H, Stoltenberg M, Vogel U, Doering P, Larsen A, Danscher G. (2007). Kupffer cells are central in the removal of nanoparticles from the organism. Part Fibre Toxicol 4:10.

Sajid M, Ilyas M, Basheer C, Tariq M, Daud M, Baig N, Shehzad F. (2015). Impact of nanoparticles on human and environment: review of toxicity factors, exposures, control strategies, and future prospects. Environ Sci Pollut Res Int 22:4122-4143.

Salykina YF, Zherdeva VV, Dezhurov SV, Wakstein MS, Shirmanova MV, Zagaynova EV, Savitsky AP. (2010). Biodistribution and clearance of quantum dots in small animals. In Sartov Fall Meeting. International Society for Optics and Photonics. 2010: 799908 .

Samia AC, Dayal S, Burda C. (2006). Quantum dot-based energy transfer: perspectives and potential for applications in photodynamic therapy. Photochem Photobiol $82: 617-625$.

Samuni Y, Ishii H, Hyodo F, Samuni U, Krishna MC, Goldstein S, Mitchell JB. (2010). 
Reactive oxygen species mediate hepatotoxicity induced by the Hsp90 inhibitor geldanamycin and its analogs. Free Radic Biol Med 48:1559-1563.

Sandoval JM, Verrax J, Vásquez CC, Calderon PB. (2012). A comparative study of tellurite toxicity in normal and cancer cells. Mol Cell Toxicol 8:327-334.

Santos SM, Dinis AM, Peixoto F, Fieerira L, Jurado AS, Videira RA. (2014). Interation of fullerene nanoparticles with biomembranes: from the partition in lipid membranes to effects on mitochondrial bioenergetics. Toxicol Sci 138:117-129.

Saukkonen JJ, Cohn DL, Jasmer RM, Schenker S, Jereb JA, Nolan CM, Peloquin CA, Gordin FM, Nunes D, Strader DB, Bernardo J, Venkataramanan R, Sterling TR, ATS (American Thoracic Society) Hepatotoxicity of Antituberculosis Therapy Subcommittee. (2006). An official ATS statement: hepatotoxicity of antituberculosis therapy. Am J Respir Crit Care Med 174:935-952.

Sayes CM, Gobin AM, Ausman KD, Mendez J, West JL, Colvin VL. (2005). Nano-C60 cytotoxicity is due to lipid peroxidation. Biomaterials 26:7587-7595.

Scheffler IE. (2001). A century of mitochondrial research: achievements and perspectives. Mitochondrion 1:3-31.

Scherer F, Anton M, Schillinger U, Henke J, Bergemann C, Kruger A, Gansbacher B, Plank C. (2002). Magnetofection: enhancing and targeting gene delivery by magnetic force in vitro and in vivo. Gene Ther 9:102-109.

Shank KE, Vetter RJ, Ziemer PL. (1977). Uptake and distribution of cadmium in mice following repeated administrations. Arch Environ Contam Toxicol 6:63-68.

Shi H, Magaye R, Castranova V, Zhao J. (2013). Titanium dioxide nanoparticles: a review of current toxicological data. Part Fibre Toxicol 10:15-8977-10-15. 
Shih CM, Ko WC, Wu JS, Wei YH, Wang LF, Chang EE, Lo TY, Cheng HH, Chen CT. (2004). Mediating of caspase-independent apoptosis by cadmium through the mitochondria-ROS pathway in MRC-5 fibroblasts. J Cell Biochem 91:384-397.

Shiohara A, Hoshino A, Hanaki K, Suzuki K, Yamamoto K. (2004). On the cyto-toxicity caused by quantum dots. Microbiol Immunol 48:669-675.

Shvedova AA, Kisin ER, Mercer R, Murray AR, Johnson VJ, Potapovich AI, Tyurina YY, Gorelik O, Arepalli S, Schwegler-Berry D, Hubbs AF, Antonini J, Evans DE, Ku BK, Ramsey D, Maynard A, Kagan VE, Castranova V, Baron P. (2005). Unusual inflammatory and fibrogenic pulmonary responses to single-walled carbon nanotubes in mice. Am J Physiol Lung Cell Mol Physiol 289:L698-708.

Siddiqui MA, Alhadlaq HA, Ahmad J, Al-Khedhairy AA, Musarrat J, Ahamed M. (2013). Copper oxide nanoparticles induced mitochondria mediated apoptosis in human hepatocarcinoma cells. PLoS ONE 8: e69534.

Simon HU, Haj-Yehia A, Levi-Schaffer F. (2000). Role of reactive oxygen species (ROS) in apoptosis induction. Apoptosis 5:415-418.

Silva FO, Carvalho MS, Mendonça R, Macedo WA, Balzuweit K, Reiss P, Schiavon MA. (2012). Effect of surface ligands on the optical properties of aqueous soluble CdTe quantum dots. Nanoscale Res Lett 7:536-546.

Slee EA, Adrain C, Martin SJ. (1999). Serial killers: ordering caspase activation events in apoptosis. Cell Death Differ 6:1067-1074.

Smith AM, Dave S, Nie S, True L, Gao X. (2006). Multicolor quantum dots for molecular diagnostics of cancer. Expert Rev Mol Diagn 6:231-244.

Smith AM, Duan H, Mohs AM, Nie S. (2008). Bioconjugated quantum dots for in vivo 
molecular and cellular imaging. Adv Drug Deliv Rev 60:1226-1240.

So MK, Xu C, Loening AM, Gambhir SS, Rao J. (2006). Self-illuminating quantum dot conjugates for in vivo imaging. Nat Biotechnol 24:339-343.

Stajn A, Zikic RV, Ognjanovic B, Saicic ZS, Pavlovic SZ, Kostic MM, Petrovic VM. (1997). Effect of cadmium and selenium on the antioxidant defense system in rat kidneys. Comp Biochem Physiol C Pharmacol Toxicol Endocrinol 117:167-172.

Stark WJ. (2011). Nanoparticles in biological systems. Angew Chem Int Ed Engl $50: 1242-1258$.

Stenvall A, Larsson E, Strand SE, Jonsson BA. (2014). A small-scale anatomical dosimetry model of the liver. Phys Med Biol 59:3353-3371.

Stern ST, McNeil SE. (2008). Nanotechnology safety concerns revisited. Toxicol Sci 101:4-21.

Stohs SJ, Bagchi D, Hassoun E, Bagchi M. (2000). Oxidative mechanisms in the toxicity of chromium and cadmium ions. J Environ Pathol Toxicol Oncol 19:201-213.

St-Pierre J, Drori S, Uldry M, Silvaggi JM, Rhee J, Jager S, Handschin C, Zheng KN, Lin JD, Yang WL, Simon DK, Bachoo R, Spiegelman BM. (2006). Suppression of reactive oxygen species and neurodegeneration by the PGC-1 transcriptional coactivators. Cell 127:397-408.

Sturgill MG, Lambert GH. (1997). Xenobiotic-induced hepatotoxicity: mechanisms of liver injury and methods of monitoring hepatic function. Clin Chem 43:15121526.

Su Y, He Y, Lu H, Sai L, Li Q, Li W, Wang L, Shen P, Huang Q, Fan C. (2009). The 
cytotoxicity of cadmium based, aqueous phase - synthesized, quantum dots and its modulation by surface coating. Biomaterials 30:19-25.

Su Y, Peng F, Jiang Z, Zhong Y, Lu Y, Jiang X, Huang Q, Fan C, Lee ST, He Y. (2011). In vivo distribution, pharmacokinetics, and toxicity of aqueous synthesized cadmium-containing quantum dots. Biomaterials 32:5855-5862.

Sun L, Li Y, Liu X, Jin M, Zhang L, Du Z, Guo C, Huang P, Sun Z. (2011). Cytotoxicity and mitochondrial damage caused by silica nanoparticles. Toxicol In Vitro 25:1619-1629.

Tang M, Wang M, Xing T, Zeng J, Wang H, Ruan DY. (2008). Mechanisms of unmodified CdSe quantum dot-induced elevation of cytoplasmic calcium levels in primary cultures of rat hippocampal neurons. Biomaterials 29:4383-4391.

Tang Y, Han S, Liu H, Chen X, Huang L, Li X, Zhang J. (2013). The role of surface chemistry in determining in vivo biodistribution and toxicity of $\mathrm{CdSe} / \mathrm{ZnS}$ coreshell quantum dots. Biomaterials 34:8741-8755.

Thomas K, Aguar P, Kawasaki H, Morris J, Nakanishi J, Savage N. (2006). Research strategies for safety evaluation of nanomaterials, part VIII: International efforts to develop risk-based safety evaluations for nanomaterials. Toxicol Sci 92:23-32.

Tiwari DK, Jin T, Behari J. (2011). Bio-distribution and toxicity assessment of intravenously injected anti-HER2 antibody conjugated CdSe/ZnS quantum dots in Wistar rats. Int J Nanomedicine 6:463-475.

Treinen-Moslen M. (2001). Toxic responses of the liver. In: The Basic Science of Poisons. Cassarett and Doull's Toxicology. (Klassen CD, Ed.). McGraw Hill, New York. 471-489. 
Turrens JF. (1997). Superoxide production by the mitochondrial respiratory chain. Biosci Rep 17:3-8.

Tzirogiannis KN, Panoutsopoulos GI, Demonakou MD, Hereti RI, Alexandropoulou KN, Basayannis AC, Mykoniatis MG. (2003). Time-course of cadmium-induced acute hepatotoxicity in the rat liver: the role of apoptosis. Arch Toxicol 77:694-701.

Uhlar CM, Whitehead AS. (1999). The kinetics and magnitude of the synergistic activation of the serum amyloid A promoter by IL-1 beta and IL- 6 is determined by the order of cytokine addition. Scand J Immunol 49:399-404.

Uhlar CM, Whitehead AS. (1999). Serum amyloid A, the major vertebrate acute-phase reactant. Eur J Biochem 265:501-523.

Venugopal R, Jaiswal AK. (1996). Nrf1 and Nrf2 positively and c-Fos and Fra1 negatively regulate the human antioxidant response element-mediated expression of NAD(P)H:quinone oxidoreductase1 gene. Proc Natl Acad Sci U S A 93:1496014965.

WadaT, Penninger JM. (2004). Mitogen-activated protein kinases in apoptosis regulation. Oncogene 23:2838-2849.

Wang B, He X, Zhang Z, Zhao Y, Feng W. (2013). Metabolism of nanomaterials in vivo: blood circulation and organ clearance. Acc Chem Res 46:761-769.

Wang J, Zhou G, Chen C, Yu H, Wang T, Ma Y, Jia G, Gao Y, Li B, Sun J, Li Y, Jiao F, Zhao Y, Chai Z. (2007). Acute toxicity and biodistribution of different sized titanium dioxide particles in mice after oral administration. Toxicol Lett 168:176185.

Wang SS, Chen L, Xia SK. (2007). Cadmium is acutely toxic for murine hepatocytes: 
effects on intracellular free $\mathrm{Ca}^{2+}$ homeostasis. Physiol Res 56:193-201.

Wang XY, Ren XF, Kahen K, Hahn MA, Rajeswaran M, Maccagnano-Zacher S, Silcox J, Cragg GE, Efros AL, Krauss TD. (2009). Non-blinking semiconductor nanocrystals. Nature 459:686-689.

Wang Y, Fang J, Leonard SS, Rao KM. (2004). Cadmium inhibits the electron transfer chain and induces reactive oxygen species. Free Radic Biol Med 36:1434-1443.

Wen H, Zhong J, Shen B, Gan T, Fu C, Zhu Z, Li R, Yang X. (2013). Comparative study of the cytotoxicity of the nanosized and microsized tellurium powders on HeLa cells. Front Biol 8: 444-450.

Will Y, Hynes J, Ogurtsov VI, Papkovsky DB. (2006). Analysis of mitochondrial function using phosphorescent oxygen-sensitive probes. Nat Protoc 1:2563-2572.

Wijnhoven SWP. (2009). Nano-silver - A review of available data and knowledge gaps in human and environmental risk assessment. Nanotoxicology 3(2):109-38.

Wojtczak L, Zablocki K. (2008). Mitochondria in cell life, death and disease. Postepy Biochem 54:129-141.

Wright DC, Han DH, Garcia-Roves PM, Geiger PC, Jones TE, Holloszy JO. (2007). Exercise-induced mitochondrial biogenesis begins before the increase in muscle PGC-1alpha expression. J Biol Chem 282:194-199.

Wu T, Tang M. (2014). Toxicity of quantum dots on respiratory system. Inhal Toxicol 26:128-139.

Wu X, Liu H, Liu J, Haley KN, Treadway JA, Larson JP, Ge N, Peale F, Bruchez MP. (2003). Immunofluorescent labeling of cancer marker Her2 and other cellular targets with semiconductor quantum dots. Nat Biotechnol 21:41-46. 
Wu YW, Li XQ, Steel D, Gammon D, Sham LJ. (2004). Coherent optical control of semiconductor quantum dots for quantum information processing. Phys E Low Dimens Syst Nanostruct 25:242-248.

Xia T, Kovochich M, Brant J, Hotze M, Sempf J, Oberley T, Sioutas C, Yeh JI, Wiesner MR, Nel AE. (2006). Comparison of the abilities of ambient and manufactured nanoparticles to induce cellular toxicity according to an oxidative stress paradigm. Nano Lett 6:1794-1807.

Xia T, Li N, Nel AE. (2010). Potential health impact of nanoparticles. Annu Rev Public Health 30:137-150.

Xu M, Deng G, Liu S, Chen S, Cui D, Yang L, Wang Q. (2010). Free cadmium ions released from CdTe-based nanoparticles and their cytotoxicity on Phaeodactylum tricornutum. Metallomics 2:469-473.

Yang RS, Chang LW, Wu JP, Tsai MH, Wang HJ, Kuo YC, Yeh TK, Yang CS, Lin P. (2007). Persistent tissue kinetics and redistribution of nanoparticles, quantum dot 705, in mice: ICP-MS quantitative assessment. Environ Health Perspect 115:1339-1343.

Yang J, Liu X, Bhalla K, Kim CN, Ibrado AM, Cai J, Peng TI, Jones DP, Wang X. (1997). Prevention of apoptosis by Bcl-2: release of cytochrome c from mitochondria blocked. Science 275:1129-1132.

Ye L, Yong KT, Liu L, Roy I, Hu R, Zhu J, Cai H, Law WC, Liu J, Wang K, Liu J, Liu Y, Hu Y, Zhang X, Swihart MT, Prasad PN. (2012). A pilot study in non-human primates shows no adverse response to intravenous injection of quantum dots. Nat Nanotechnol 7:453-458. 
Yeh JH, Huang CC, Yeh MY, Wang JS, Lee JK, Jan CR. (2009). Cadmium-induced cytosolic $\mathrm{Ca}^{2+}$ elevation and subsequent apoptosis in renal tubular cells. Basic Clin Pharmacol Toxicol 104:345-351.

Yeh TK, Wu JP, Chang LW, Tsai MH, Chang WH, Tsai HT, Yang CS, Lin P. (2011). Comparative tissue distributions of cadmium chloride and cadmium-based quantum dot 705 in mice: Safety implications and applications. Nanotoxicology 5:91-97.

Yin Y, Alivisatos AP. (2005). Colloidal nanocrystal synthesis and the organic-inorganic interface. Nature 437:664-670.

Yong KT. (2010). Biophotonics and biotechnology in pancreatic cancer: cyclic RGDpeptide-conjugated type II quantum dots for in vivo imaging. Pancreatology 10:553-564.

Yong KT, Hu R, Roy I, Ding H, Vathy LA, Bergey EJ, Mizuma M, Maitra A, Prasad PN. (2009). Tumor targeting and imaging in live animals with functionalized semiconductor quantum rods. ACS Appl Mater Interfaces 1:710-719.

Yong KT, Law WC, Hu R, Ye L, Liu L, Swihart MT, Prasad PN. (2013). Nanotoxicity assessment of quantum dots: from cellular to primate studies. Chem Soc Rev 42:1236-1250.

Yong KT, Wang Y, Roy I, Rui H, Swihart MT, Law WC, Kwak SK, Ye L, Liu J, Mahajan SD, Reynolds JL. (2012). Preparation of quantum dot/drug nanoparticle formulations for traceable targeted delivery and therapy. Theranostics 2:681-694.

You Q, Cheng L, Reilly TP, Wegmann D, Ju C. (2006). Role of neutrophils in a mouse model of halothane-induced liver injury. Hepatology 44:1421-1431. 
Zhang Y, Chen W, Zhang J, Liu J, Chen G, Pope C. (2007). In vitro and in vivo toxicity of CdTe nanoparticles. J Nanosci Nanotechnol 7:497-503.

Zhao JL, Bardecker JA, Munro AM, Liu MS, Niu YH, Ding IK, Luo JD, Chen BQ, Jen AKY, Ginger DS. (2006). Efficient CdSe/CdS quantum dot light-emitting diodes using a thermally polymerized hole transport layer. Nano Lett 6:463-467.

Zhu Y, Hong H, Xu ZP, Li Z, Cai W. (2013). Quantum dot-based nanoprobes for in vivo targeted imaging. Curr Mol Med 13:1549-1567.

Zhu Y, Zhao Q, Li Y, Cai X, Li W. (2006). The interaction and toxicity of multi-walled carbon nanotubes with Stylonychia mytilus. J Nanosci Nanotechnol 6:1357-1364.

Zoratti M, Szabo I. (1995). The mitochondrial permeability transition. Biochim Biophys Acta 1241:139-176.

Zorov DB, Krasnikov BF, Kuzminova AE, Vysokikh MY, Zorova LD. (1997). Mitochondria revisited. Alternative functions of mitochondria. Biosci Rep 17:507-520.

Zrazhevskiy P, Sena M, Gao X. (2010). Designing multifunctional quantum dots for bioimaging, detection, and drug delivery. Chem Soc Rev 39:4326-4354. 